\title{
Thermal Performance of Fire Resistive Materials III. Fire Test on a Bare Steel Column
}

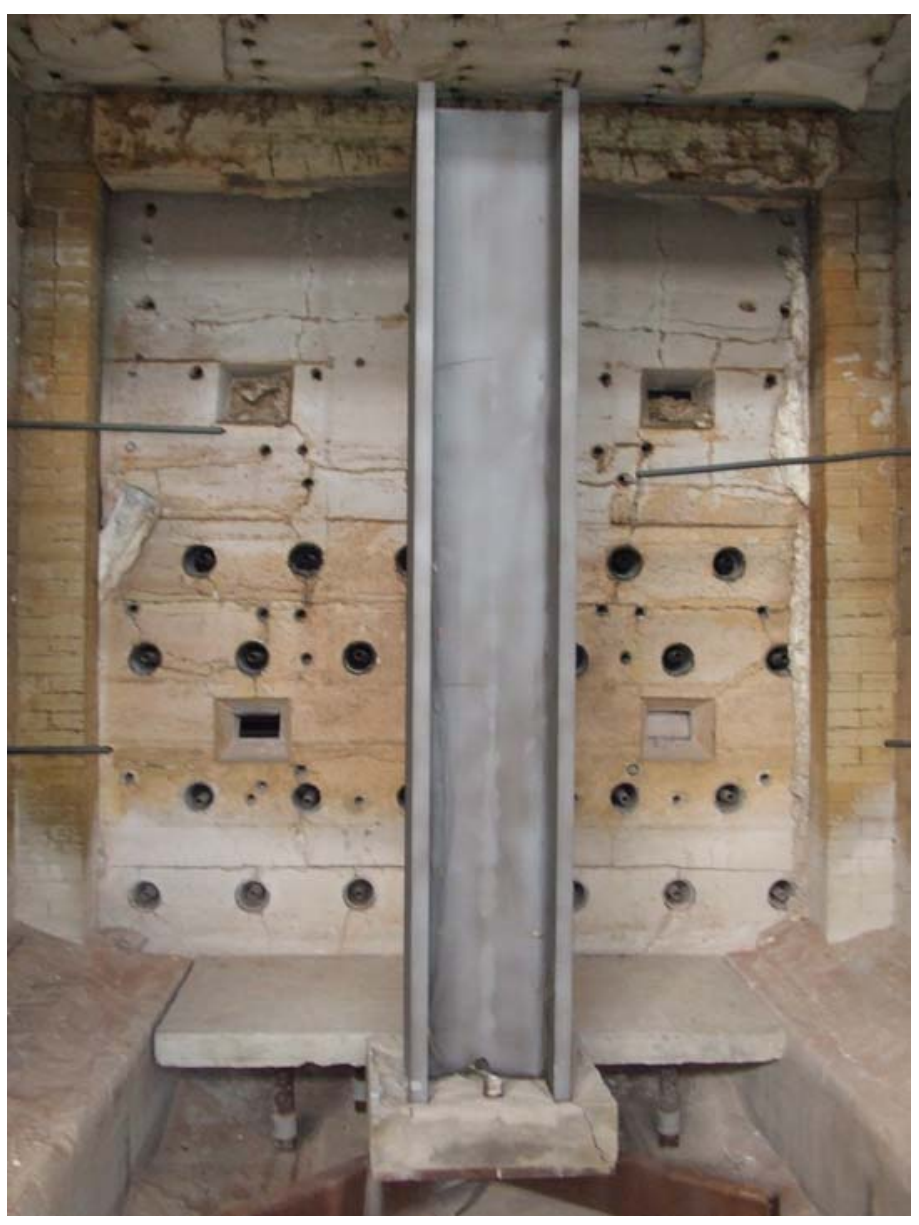

Dale P. Bentz Leonard M. Hanssen Boris Wilthan

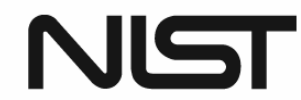

National Institute of Standards and Technology U.S. Department of Commerce 


\section{Thermal Performance of Fire Resistive Materials III. Fire Test on a Bare Steel Column}

Dale P. Bentz

Building and Fire Research Laboratory National Institute of Standards and Technology

Gaithersburg, MD 20899-8615

Dr. Leonard M. Hanssen and Boris Wilthan

Physics Laboratory National Institute of Standards and Technology Gaithersburg, MD 20899-8442

April 2009

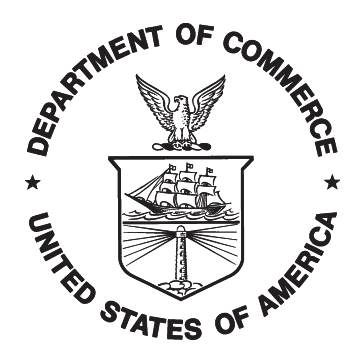

U.S. Department of Commerce Gary Locke, Secretary

National Institute of Standards and Technology Patrick D. Gallagher, Deputy Director 


\begin{abstract}
This report is part III in an ongoing series concerning the characterization and modeling of the thermal performance of fire resistive materials (FRMs). In part I, a methodology for characterizing FRMs to provide quantitative material property inputs for thermal performance models was outlined, and in part II, one such multi-layer model was demonstrated for simulating the results of high temperature slug calorimeter experiments conducted in a small furnace. Attempts to extend the one-dimensional model to predict the performance of FRM-insulated steel columns in a standard ASTM International E119 fire exposure [1] were successful only when a safety (viewing) factor of approximately 0.5 was introduced for reducing the radiative heat transfer between the fire and the protected column. To justify this fitted value of the viewing factor, a simpler fire test of a single bare W14x233 structural steel column was conducted in the column furnace at a commercial U.S.-based testing laboratory. This report presents the results of that test, along with an analysis based on a previously developed general heat balance equation [2]. The emissivity value that provides the best fit to the experimental data is contrasted against the measured emissivity of the structural steel. The resultant safety factor is found to be on the order of 0.45 , in general agreement with the value used previously in the onedimensional thermal model. One unique feature of this study is that data was also collected during the (slow) cool down period that followed the termination of the fire exposure, allowing a comparison of the convective heat transfer coefficients during a fire exposure and subsequent cooling in a furnace environment. Additional thermocouples were placed on the steel column and the furnace walls and two plate thermometers were placed near the surface of the steel column to better characterize the furnace fire environment. All of the measured data is provided in an Appendix to place it in the public domain where it may serve as a reference data set for other researchers.
\end{abstract}




\section{Table of Contents}

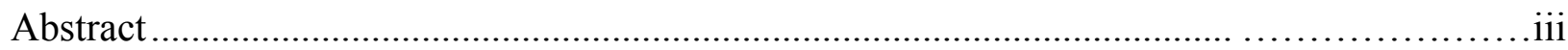

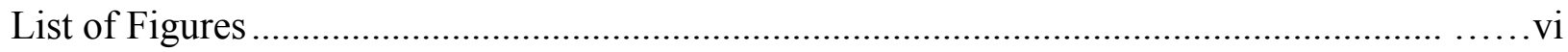

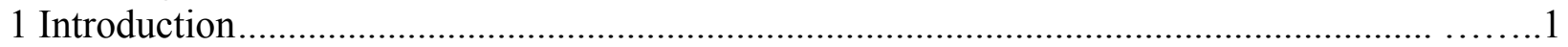

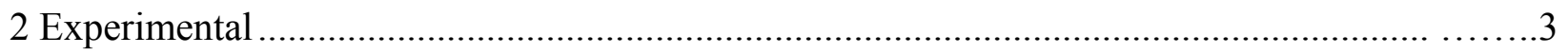

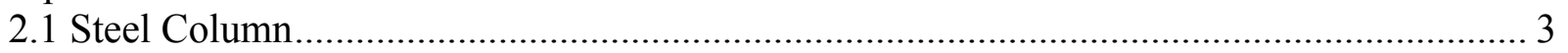

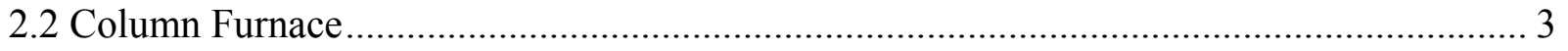

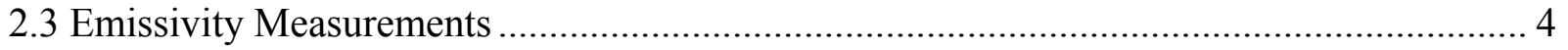

3 Results and Analysis ....................................................................................... 9

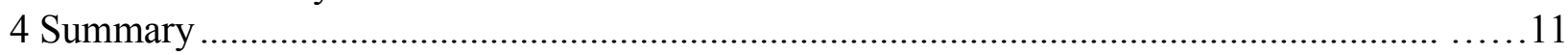

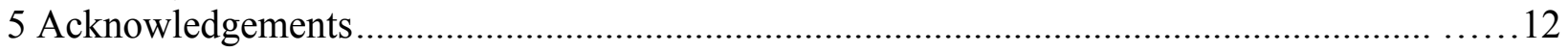

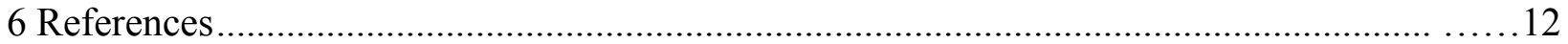

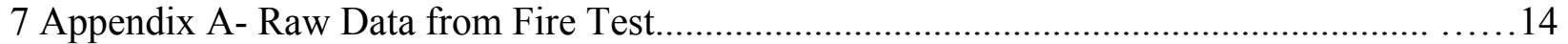

7.1 Time and Furnace Temperature Data ...................................................................... 14

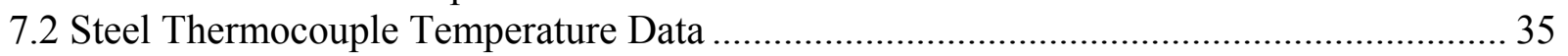

7.3 Wall Thermocouple and Plate Thermometer Data ..................................................... 60 


\section{List of Figures}

Figure 1. Schematic of the cross-section of the W14x233 structural steel column tested in the column furnace (not to scale). Units are given in inches to conform to specification documents for columns.

Figure 2. Thermocouple layout for the bare steel column tested in the column furnace.

Dimensions are given in feet and inches to conform to specification documents for columns.

Figure 3. Structural steel specimen in specimen holder for infrared spectral-emissivity measurement.

Figure 4. Measured emittance results vs. wavelength at various temperatures for the structural steel specimen that was sandblasted (prior to fire exposure) ............................................ 7

Figure 5. Computed emissivity vs. temperature for the sandblasted steel specimen................... 8

Figure 6. Experimental and model results for average temperature of bare steel column vs. time during an ASTM International E119 fire test exposure............................................. 9

Figure 7. Measured and predicted temperatures during fire exposure and subsequent cool down

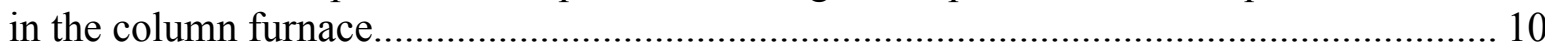




\section{Introduction}

Standardized fire testing is an integral part of the overall design process for providing buildings and structures with the requisite fire resistance. It would therefore be of great interest to material manufacturers, designers and architects, and forensics engineers to have quantitative and reliable tools to model the performance of steel structures protected with a fire resistive material (FRM) in both real and test fire conditions. In part I of this series [3], the necessary characterization of the FRMs and substrate materials was presented. In part II [4], the details of a one-dimensional multi-layer heat transfer model that was originally developed to model the thermal performance of fire fighters' clothing [5] were presented and the model was verified by comparison against a variety of slug calorimeter experiments [6] performed previously in the Building and Fire Research Laboratory (BFRL) at the National Institute of Standards and Technology (NIST). In extending this one-dimensional model to ASTM International E119 [1] fires for four FRM-insulated steel columns of different dimensions and/or different FRM thicknesses, adequate agreement between model and real steel temperature data was only obtained when a safety (configuration or viewing) factor of approximately 0.5 was introduced as a multiplicative factor for reducing the radiative heat transfer between the gas fire and the FRMinsulated steel column. To justify this choice of safety factor, an E119 fire test on a single bare steel column was conducted at the same commercial U.S.-based testing laboratory in the same column furnace where the four data sets for FRM-insulated steel had been produced. The results of that test, along with emissivity measurements for the structural steel performed at NIST, form the basis for the part III report in this series.

Applying a similar approach, Kay, Kirby, and Preston determined a best-fit safety factor of 0.45 for beam and column test furnaces in the U.K. and the Netherlands, when the emissivities of the fire and steel were both set at 0.8 [7]. They correctly pointed out that this safety factor is in reality a "curve-fitting factor chosen to ensure....the prediction of a heating rate in agreement with that observed in nationally approved fire-resistance-testing furnaces" [7]. It is important to note that this viewing or safety factor may be totally different for real fires than that found for furnace testing. For example, Kirby has found that using a safety factor of 1.0 provides reasonable estimates of the performance of structural steelworks in real building fires [8]. The best-fit safety factor for a furnace will be a function of furnace geometry as well as the type of fuel being burnt [7], and would thus be expected to vary significantly from one furnace to another. For that reason, in this study, the bare steel column was tested in the same column furnace in which the four FRM-insulated steel columns had been previously evaluated some years ago.

In the literature, there are a variety of analytical tools available for predicting the performance of a bare steel column in a furnace fire test. Wong and Ghojel have developed a spreadsheet that is available for free downloading that implements both a standard analysis as employed in the Eurocodes as well as one based on gas combustion theory for radiation exchange between a gas body and its enclosure [9-11]. The standard analysis is similar to that presented by Parkinson and Kodur [2], from which the following equation can be used to calculate the temperature rise, $\Delta T_{s}$, of the steel column during a time interval, $\Delta t$ : 


$$
\Delta T_{s}=\frac{F}{\rho_{s} C_{p s}}\left\{h_{c}\left(T_{f}-T_{s}\right)+\sigma \varepsilon\left(T_{f}^{4}-T_{s}^{4}\right)\right\} \Delta t
$$

where:

$F=$ section factor $=$ column perimeter $/$ column area, $\mathrm{m}^{-1}$,

$\rho_{S}=$ steel density, taken to be $7860 \mathrm{~kg} / \mathrm{m}^{3}$,

$C_{p s}=$ specific heat of steel, $\mathrm{J} /(\mathrm{kg} \cdot \mathrm{K})$,

$h_{c}=$ convective heat transfer coefficient, generally taken to be $25 \mathrm{~W} /\left(\mathrm{m}^{2} \cdot \mathrm{K}\right)[2,7,9,10]$,

$T_{f}=$ furnace temperature at time $\mathrm{t}, \mathrm{K}$,

$T_{s}=$ steel temperature at time $\mathrm{t}, \mathrm{K}$,

$\sigma=$ Stefan-Boltzmann constant, $5.67 \times 10^{-8} \mathrm{~W} /\left(\mathrm{m}^{2} \cdot \mathrm{K}^{4}\right)$,

$\varepsilon=$ effective emissivity for radiative heat transfer,

$\Delta t=$ time interval of computation, $\mathrm{s}$.

This equation indicates that there are two contributions to heat transfer between the fire and the steel column, a convective component due to the presence of hot gases flowing past the steel surfaces and a radiative component indicating transfer of radiation from the hot furnace (walls) and fire to the steel. Since the radiative term depends on the difference in temperatures first raised to the fourth power, it generally dominates over the convective term at higher temperatures and/or during the latter stages of a standard fire exposure test.

The appropriate value to use for the single emissivity term $\varepsilon$ will depend on both the location of the steel column within the furnace and its size relative to the interior volume of the furnace [7]. In this study, this effective emissivity will be calibrated to the experimental data and utilized to determine a safety factor for this column/furnace combination from:

$$
\varepsilon=\gamma \varepsilon_{s}
$$

where:

$\gamma=$ safety (or viewing) factor for this column/furnace combination and

$\varepsilon_{s}=$ measured emissivity value for structural steel.

The major assumptions of equation (1) are that the thermal conductivity of the steel is high enough that the steel temperature is uniformly distributed throughout the steel cross section and that $T_{f}$ is the appropriate source temperature to be used both for radiative and convective heat transfer computations. Typically, the specific heat of the steel is a function of temperature. In this study, the following equation is used to describe this relationship [12]:

$$
C_{p s}=454.2+(0.1686) T_{s C}+(0.000532) T_{S C}^{2}
$$

with $T_{s C}$ being the steel temperature in ${ }^{\circ} \mathrm{C}$. 


\section{Experimental}

\subsection{Steel Column}

A single W14x233 structural steel column with a height of $0.25 \mathrm{~m}$ (99") was tested. The W14x233 designation specifies the column geometry, as detailed in Figure 1. The steel column was sandblasted with silica sand prior to testing, effectively removing any oxide layer that had formed previously. Based on the metric equivalents of the dimensions of the column given in Figure 1, the section factor ( $F$ in equation 1$)$ or ratio of perimeter to area is calculated to be $54 \mathrm{~m}^{-1}$.

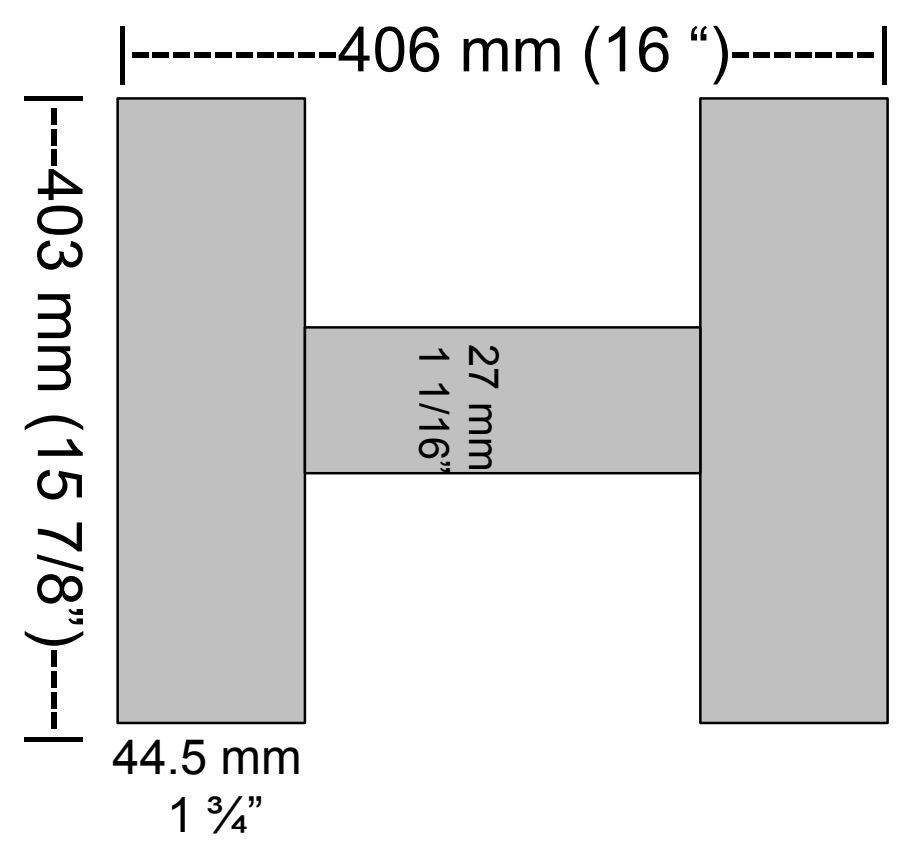

Figure 1. Schematic of the cross-section of the W14x233 structural steel column tested in the column furnace (not to scale). Units given in inches are the exact values, to conform to specification documents for columns.

\subsection{Column Furnace}

The column furnace, as depicted on the cover page of the report, has been in operation for many years at a commercial U.S.-based testing laboratory. The walls on all four sides of the furnace contain a number of entry ports to provide the natural gas that is used to create the fire environment. For this study, the normal thermocouple layouts employed in the furnace and on the steel column were supplemented with a few additional thermocouples on the steel, sets of two thermocouples on two of the furnace walls (located $90^{\circ}$ from one another), and two plate thermometers located approximately $100 \mathrm{~mm}$ (4") from the steel column (flange) surfaces at mid-height in the furnace. The thermocouples were mounted on the walls to provide a 
comparison of wall and fire temperatures. The plate thermometers were installed to provide an additional data set comparing their performance to those of the standard furnace thermocouples, which may be of use to other fire research groups considering a switch to plate thermometers.

The layout for the thermocouples on the steel column is provided in Figure 2. During the test, particularly for the cool down portion, thermocouples \#5 and \#18 did not provide reliable temperature indications for unknown reasons; based on this, they were eliminated from being included in the analysis. Thermocouples \#19 and \#20 were located on the north wall of the furnace, while \#21 and \#22 were located on the west wall of the furnace. Finally, the plate thermometer readings were recorded as channels \#23 and \#24. Readings for the thermocouples and plate thermometers were recorded once per minute during the first $823 \mathrm{~min}$ of the $75 \mathrm{~min}$ fire exposure and the subsequent cool down period, and then once per 5 min after that out to a total time of $4123 \mathrm{~min}$. A set of eight thermocouples inserted through the side walls (see cover photo) were utilized to assess (and control) the furnace temperature.

\subsection{Emissivity Measurements}

Kay et al. have previously summarized measured emissivity values for dull oxidized mild steel [7]. Based on data from five different sources, their general conclusion is that "it would seem appropriate to adopt a value of 0.8 for $\varepsilon$ across the temperature range $20^{\circ} \mathrm{C}$ to $800{ }^{\circ} \mathrm{C}$ [7]." Four of the five data sets exhibited little variation in $\varepsilon$ with temperature in the range of $20^{\circ} \mathrm{C}$ to $600{ }^{\circ} \mathrm{C}$. For the present study, two pieces of the steel, cubes approximately $50 \mathrm{~mm}(2$ ") in dimension, were extracted from a top corner of the column, one before and one after the fire testing. The reflectances of the two specimens were measured at room temperature using a commercial device $[13,14]$. The device is first calibrated using two cylindrical reflectance standards, a gold surface with a reference reflectance of 0.955 and a black surface (opposite side of the gold reference material covered with a black coating) with a reference value of 0.089 . Measurements at $23{ }^{\circ} \mathrm{C}$ on two sides of the steel cube that had been exposed to the fire yielded an average reflectance of 0.15 with a standard deviation of 0.01 . This would correspond to an emissivity of 0.85 , in reasonable agreement with the previous data summary by Kay et al. [7]. For the blasted steel surface, an average reflectance of 0.68 (emissivity of 0.32 ) with a standard deviation of 0.01 was obtained.

Further characterization of the steel emissivity as a function of wavelength and temperature was performed on two systems at the NIST Fourier Transform Infrared Spectrophotometry laboratory. Cylindrical disks, $19 \mathrm{~mm}$ in diameter and $5 \mathrm{~mm}$ in thickness, were extracted from one of the exposed surfaces of each steel cube. Near-normal directionalhemispherical reflectance (DHR) measurements were performed at room temperature (RT, $21^{\circ} \mathrm{C}$ ), using the Reference Infrared Integrating Sphere Reflectometer (RIISR), using a Fourier transform spectrometer source, as described in $[15,16]$. Since the samples are opaque, by energy conservation and Kirchoff's Law, the near-normal emittance is equal to 1 minus the near-normal DHR. Measurements were performed on the RIISR over a wavelength range from about $2 \mu \mathrm{m}$ to about $15 \mu \mathrm{m}$. At elevated temperatures, the near-normal emittance (which will further be denoted as simply "the emittance") measurements were made using the Infrared Spectral Emittance Measurement (ISEM) facility, which also includes another Fourier transform 
spectrometer source, as described in $[17,18]$. Measurements were performed on the ISEM facility at nominal temperatures of $200{ }^{\circ} \mathrm{C}, 400{ }^{\circ} \mathrm{C}, 600{ }^{\circ} \mathrm{C}$, and $800{ }^{\circ} \mathrm{C}$ for wavelengths ranging from about 2 (4) $\mu \mathrm{m}$ to about 17 (24) $\mu \mathrm{m}$, depending on the temperature and detector used. Two detectors were used: a low sensitivity pyroelectric detector (to $24 \mu \mathrm{m}$ ) and a higher sensitivity cryogenically cooled $\mathrm{HgCdTe}$ detector (to $17 \mu \mathrm{m}$ to $20 \mu \mathrm{m}$ ). An image of the originally blasted

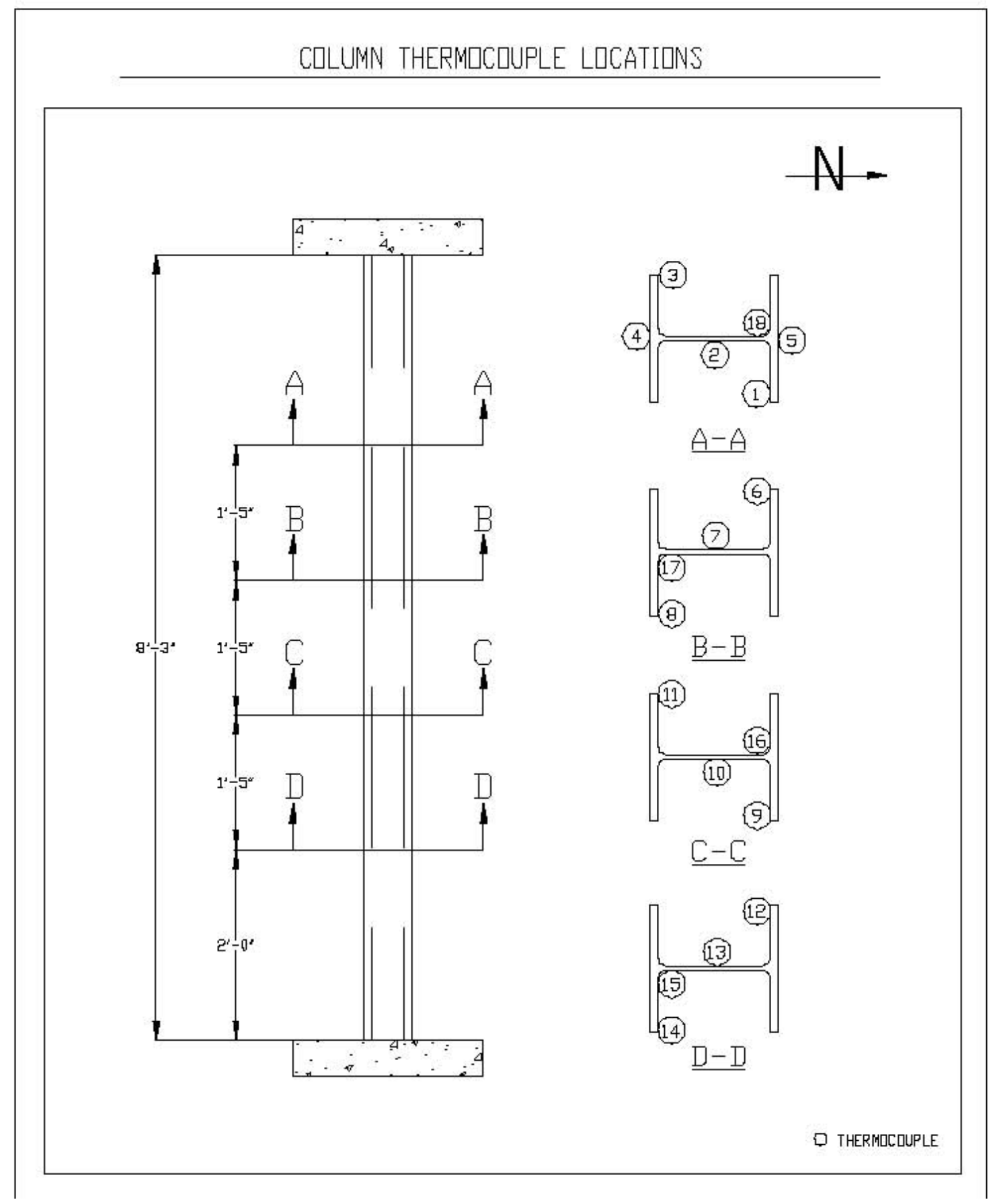

Figure 2. Thermocouple layout for the bare steel column tested in the column furnace. Dimensions are given in feet and inches to conform to specification documents for columns. 
specimen after exposure to $400{ }^{\circ} \mathrm{C}$ in its sample holder is provided in Figure 3. Figure 4 provides a summary of the data measured for the originally blasted specimen. The average expanded uncertainty of the measured reflectance, using the RIISR, was $3.5 \%$ of the reflectance value. The average expanded uncertainty in the measured emittance, using the ISEM facility, was $2.0 \%$ of the emittance value. The three heavy curves represent the RT results at beginning, middle and end of the overall sample baking process. The pairs of curves for each temperature were taken sequentially and show the cumulative effects of baking during the measurements. It can be observed in Figure 4 that measurement temperature did not have a significant influence on measured emittance as the specimen first heated to $400{ }^{\circ} \mathrm{C}$ ( or $800{ }^{\circ} \mathrm{C}$ ) exhibited similar results when measured at that temperature as it exhibited after being cooled back down to room temperature. This would be in agreement with previous results for oxidized steel [7]. The two curves for measurements at $400{ }^{\circ} \mathrm{C}$ indicate the progress of the oxidation process during exposure to this temperature, while the $200{ }^{\circ} \mathrm{C}$ results suggest that little if any oxidation had occurred by this temperature.

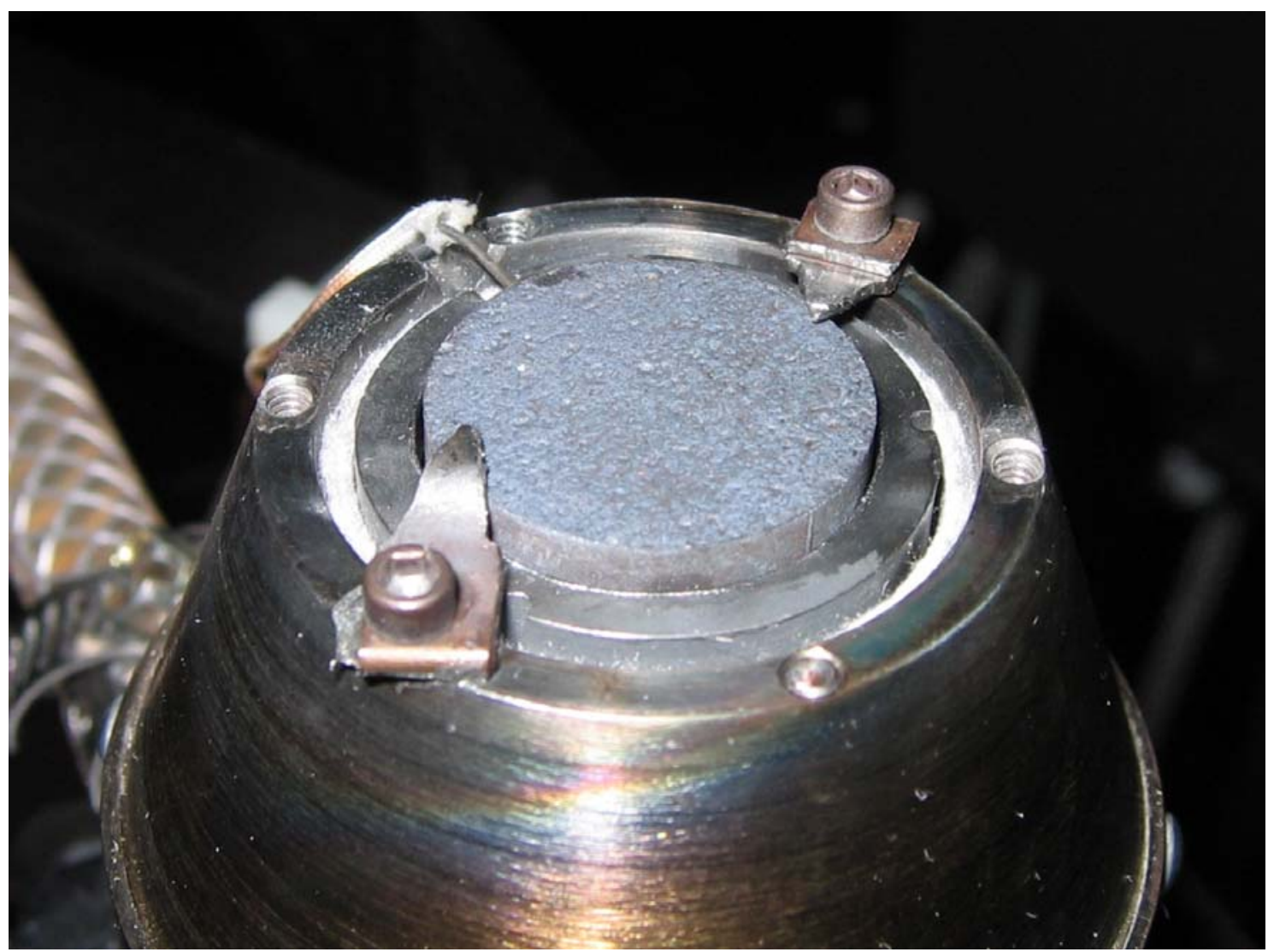

Figure 3. Structural steel specimen in specimen holder for infrared spectral-emissivity measurement.

The measured emittance values were used to compute emissivities using the procedure outlined in Holman [19]. First, the emissive power of a blackbody per unit wavelength $\left(E_{b \lambda}\right)$ is computed as:

$$
E_{b \lambda}=\frac{C_{1} \lambda^{-5}}{e^{C_{2} / \lambda T}-1}
$$


where:

$\lambda=$ wavelength, $\mu \mathrm{m}$,

$T=$ temperature, $\mathrm{K}$

$C_{l}=3.743 \times 10^{8} \mathrm{~W} \cdot \mu \mathrm{m}^{4} / \mathrm{m}^{2}$, and

$C_{2}=1.4287 \times 10^{4} \mu \mathrm{m} \cdot \mathrm{K}$. At higher temperatures, the peak of the $E_{b \lambda}$ function is shifted to shorter wavelengths [19]. Based on this function and the measured emittance $\left(\varepsilon_{\lambda}\right)$ vs. wavelength response (from Figure 4 for example), the total emissivity of the steel specimen $(\varepsilon)$ was estimated as:

$$
\varepsilon=\frac{\int_{0}^{\infty} \varepsilon_{\lambda} E_{b \lambda} d \lambda}{\int_{0}^{\infty} E_{b \lambda} d \lambda}
$$

The obtained results are provided in Figure 5. It should be noted that the estimated room temperature emissivity of 0.318 is in good agreement with the total reflectance measurement value (0.32) noted above. Clearly, once the steel undergoes oxidation, a much higher emissivity surface is produced.

\section{Sandblasted specimen prior to fire exposure}

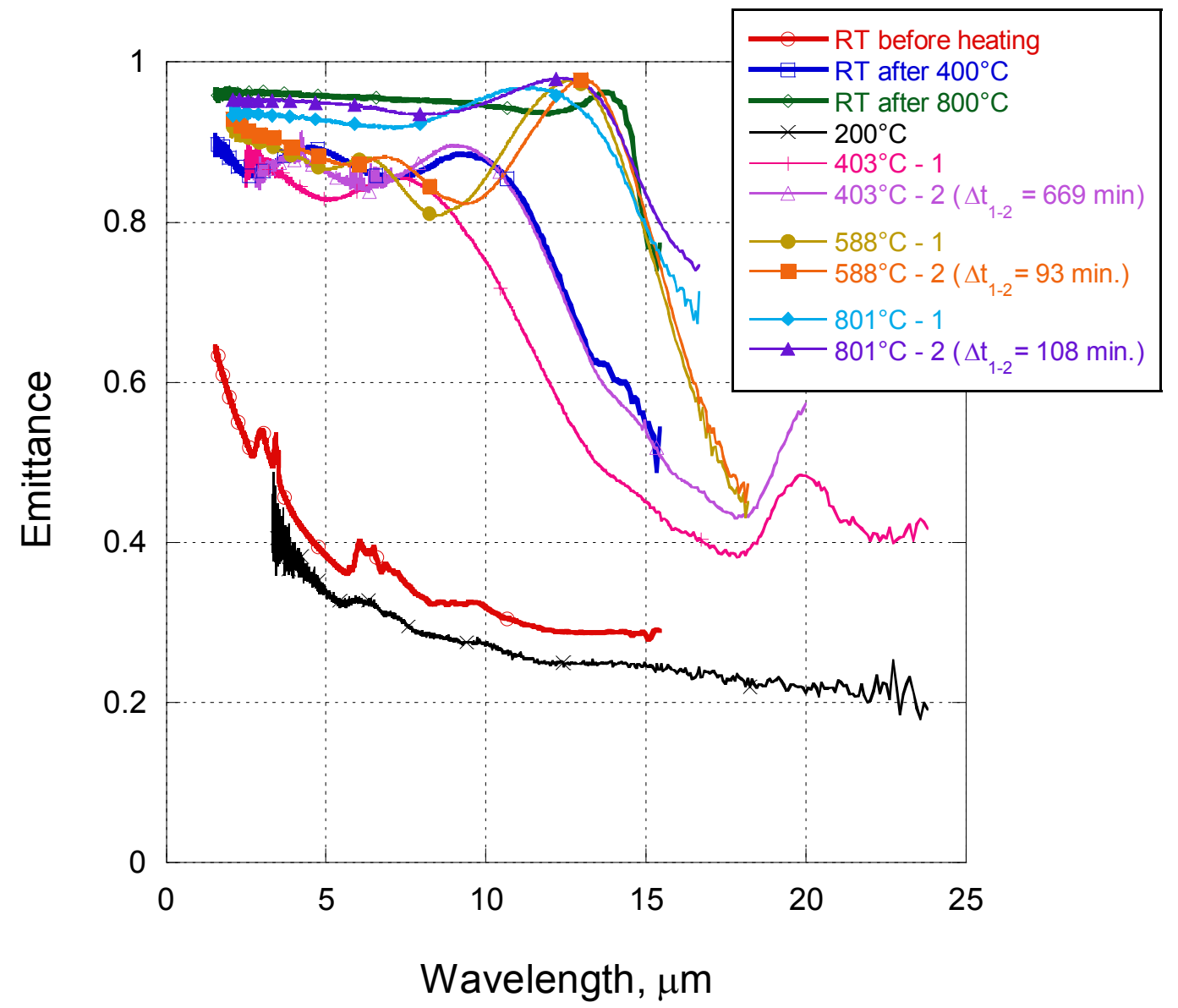

Figure 4. Measured emittance results vs. wavelength at various temperatures for the structural steel specimen that was sandblasted (prior to fire exposure). 


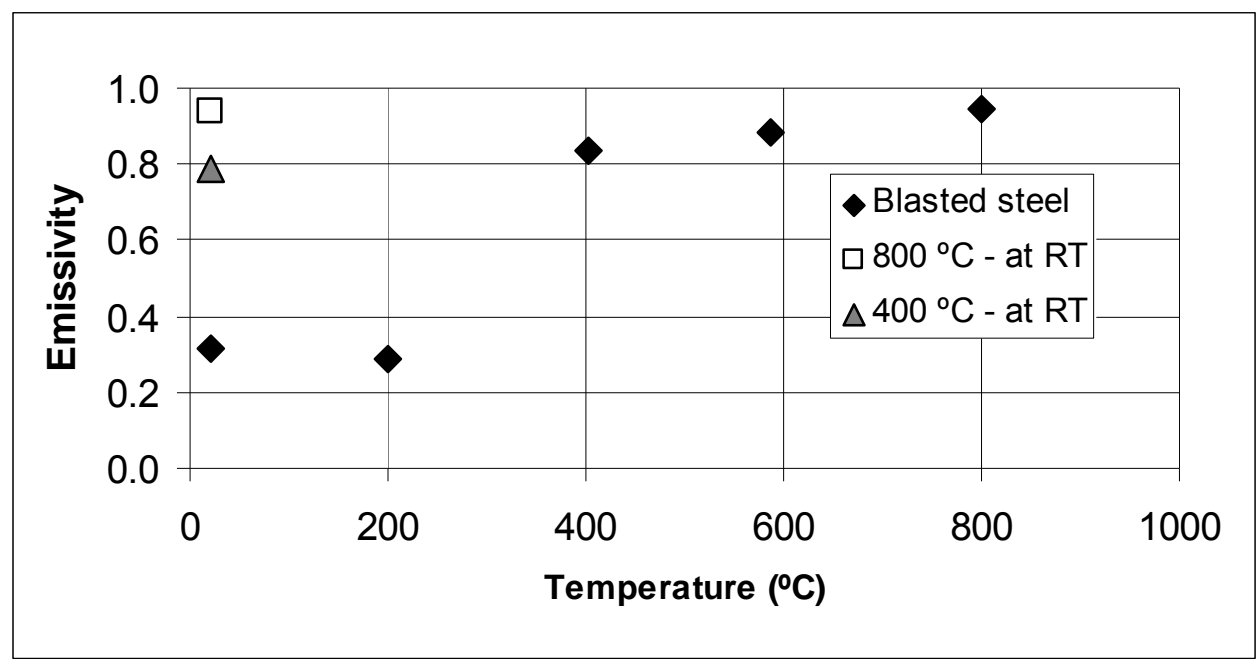

Figure 5. Computed emissivity vs. temperature for the sandblasted steel specimen. 


\section{Results and Analysis}

While the results tabulated in Appendix A indicate significant variability in the steel column temperatures depending on thermocouple height and location around the perimeter of the column, since equation (1) assumes a single steel temperature, the values for all of the steel surface thermocouples were averaged, neglecting those of thermocouples \#5 and \#18 as mentioned previously. This average value was then compared to predictions obtained using equation (1) with various values for the emissivity term, along with the previously listed values for steel density and heat capacity, convective heat transfer coefficient, and section factor. In applying equation (1), the actual measured average furnace temperatures were employed instead of the standard fire exposure curve. Results are displayed graphically in Figure 6, from which it is clear that the best single choice for effective emissivity is given by 0.4. This value was verified by executing simulations with emissivity values of $0.35,0.4$, and 0.45 , and demonstrating that the smallest average absolute error in predicted steel temperatures was produced with the value of 0.4 .

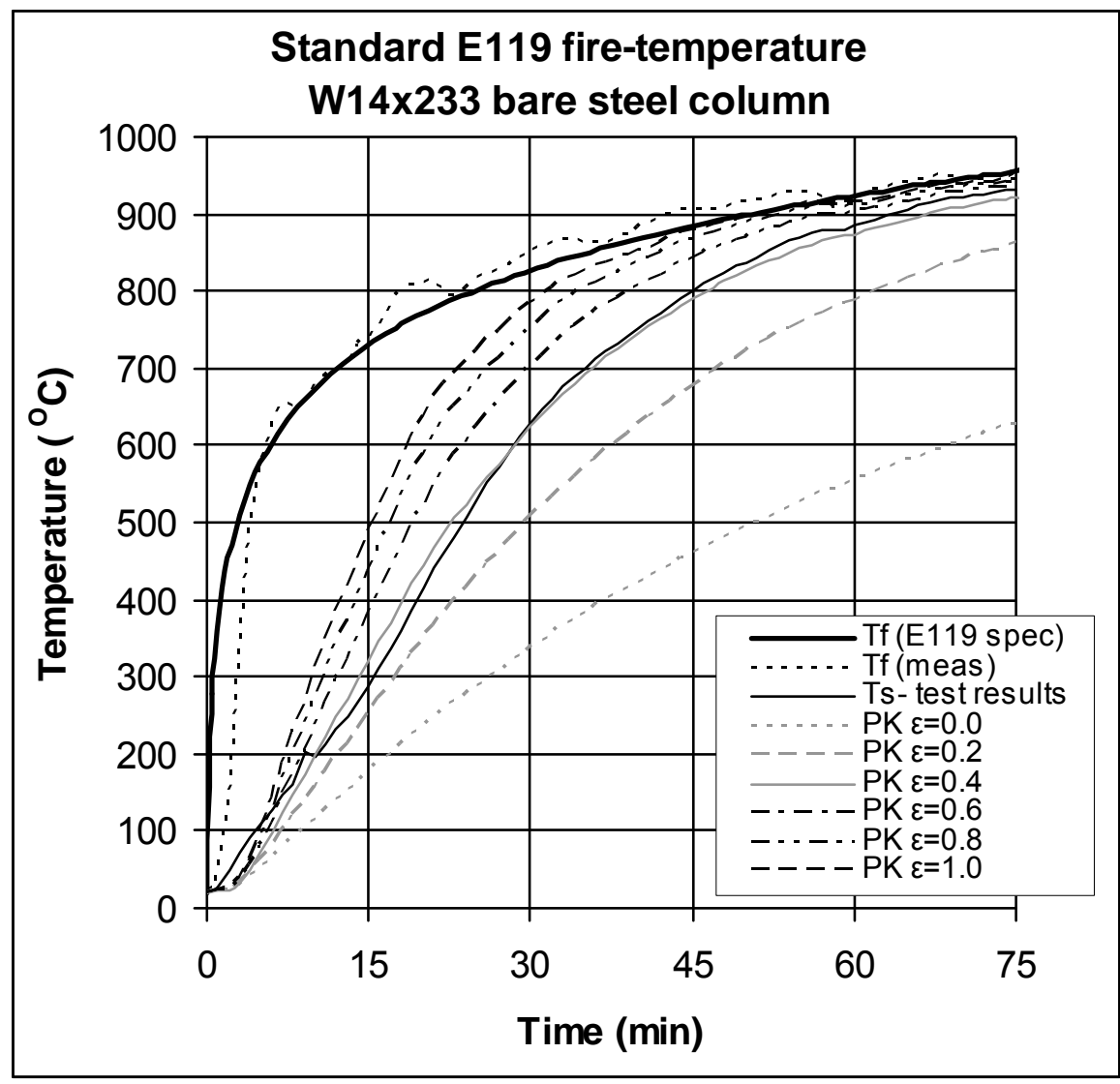

Figure 6. Experimental and model results for average temperature of bare steel column vs. time during an ASTM International E119 fire test exposure. 
With $\varepsilon=0.4$, an excellent fit to the measured data is obtained for temperatures greater than $200{ }^{\circ} \mathrm{C}$ or testing times greater than about $10 \mathrm{~min}$. Comparing this emissivity value to those measured at $400{ }^{\circ} \mathrm{C}(0.83)$ or $800{ }^{\circ} \mathrm{C}(0.95)$, one calculates a "safety" factor of between 0.42 and 0.48 , in reasonable agreement with the safety factor of about 0.5 suggested by measurements obtained previously on FRM-protected steel columns and the value of 0.45 obtained previously by Kay et al. [7].

The data obtained during the cool down period provides a further chance to characterize the radiative and convective heat transfer environments in the column furnace. During the cool down period, to slow the cooling rate, most of the vents to the furnace were closed off, suggesting a convective heat transfer coefficient value below that of $25 \mathrm{~W} /\left(\mathrm{m}^{2} \cdot \mathrm{K}\right)$ used during the fire exposure. In Figure 7, the same emissivity value of 0.4 was maintained and the convective coefficient was reduced to $10 \mathrm{~W} /\left(\mathrm{m}^{2} \cdot \mathrm{K}\right)$ to provide an adequate fit to the measured data during the cool down period. Once again, the value of 10 was verified by executing simulations with values of $5,10,15$, and 20 and demonstrating that the smallest average absolute error in steel temperature was produced for $h_{c}=10 \mathrm{~W} /\left(\mathrm{m}^{2} \cdot \mathrm{K}\right)$.

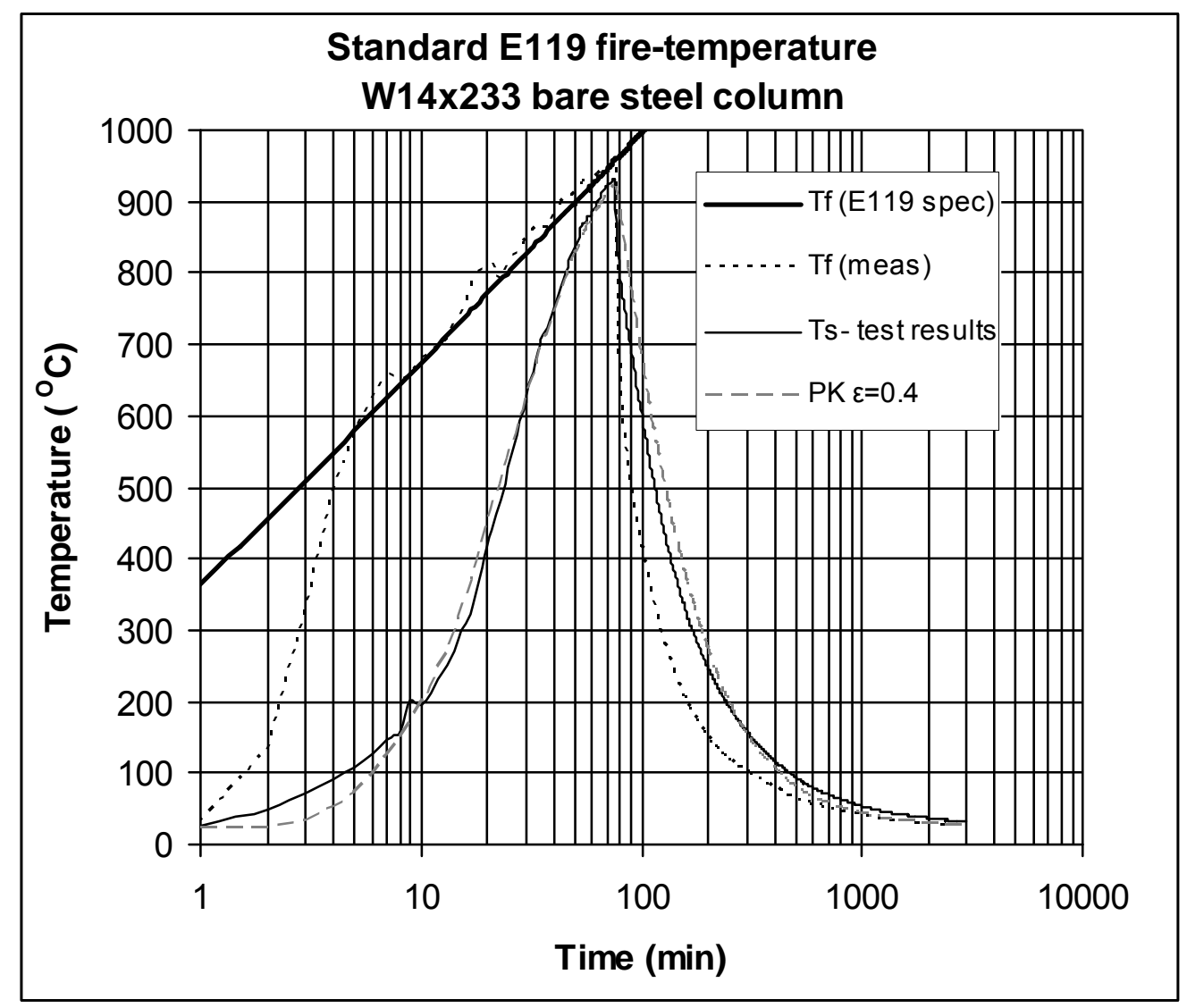

Figure 7. Measured and predicted temperatures during fire exposure and subsequent cool down in the column furnace. 


\section{Summary}

An ASTM International E119 fire test was conducted on a W14x233 bare steel column in a commercial U.S.-based testing laboratory to provide support for the previous choice of a safety factor of 0.5 for the radiative heat transfer environment in the column furnace. The obtained raw temperature data has been provided in Appendix A of this report, to provide an archive data set that may be used by others. A heat transfer analysis of the resultant temperatures has indicated a best-fit effective emissivity value of 0.4 to characterize the radiative heat transfer environment of the furnace during this test. Assuming an emissivity value in the range of 0.83 to 0.95 for the oxidized structural steel employed in this test, the results are consistent with a safety factor in the range of 0.42 to 0.48 , in general agreement with the value determined in the modeling of four FRM-insulated columns that were previously tested in the same column furnace employed in this study. This safety factor will be different for each furnace and, as suggested by Wong [11], could even be different for different size columns tested in a single furnace. 


\section{Acknowledgements}

The authors would like to acknowledge the financial and technical support of the current industrial members of the National Institute of Standards and Technology/industry consortium on Performance Assessment and Optimization of Fire Resistive Materials: the American Iron and Steel Institute, Isolatek International, PPG Industries, and W.R. Grace \& Co.- Conn. Special thanks are expressed to Isolatek International for providing the bare steel column employed in the column furnace test.

\section{References}

[1] ASTM E119, "Standard Test Methods for Fire Tests of Building Construction and Materials," ASTM Annual Book of Standards, ASTM International, West Conshohocken 2007.

[2] Parkinson, D.L., and Kodur, V.K.R., "Performance-Based Design of Structural Steel for Fire Conditions - A Calculation Methodology," Steel Structures, 7, 219-226, 2007.

[3] Bentz, D.P., and Prasad, K.R., "Thermal Performance of Fire Resistive Materials I. Characterization of Fire Resistive Materials with Respect to Thermal Performance Models," NISTIR 7401, U.S. Department of Commerce, February 2007.

[4] Prasad, K.R., and Bentz, D.P., "Thermal Performance of Fire Resistive Materials II. A MultiLayer One-Dimensional Heat Transfer Model for Fire Resistive Materials Protecting a Substrate," NISTIR 7482, U.S. Department of Commerce, February 2008.

[5] Prasad, K., Twilley, W., and Lawson, J.R., "Thermal Performance of Fire Fighters' Protective Clothing. 1. Numerical Study of Transient Heat and Water Vapor Transfer," NISTIR 6881, U.S. Department of Commerce, August 2002.

[6] Bentz, D.P., Flynn, D.R., Kim, J.H., and Zarr, R.R., "A Slug Calorimeter for Evaluating the Thermal Performance of Fire Resistive Materials," Fire and Materials, Vol. 30 (4), 257-270 (2006).

[7] Kay, T.R., Kirby, B.R., and Preston, R.R., "Calculation of the Heating Rate of an Unprotected Steel Member in a Standard Fire Resistance Test," Fire Safety Journal, 26, 327 350, 1996.

[8] Kirby, B.R., "The Temperatures Attained by Structural Steelwork in Building Fires," Interflam 2001, 419-429.

[9] Wong, M.B., and Ghojel, J.I., "Spreadsheet Method for Temperature Calculation of Unprotected Steelwork Subject to Fire," The Structural Design of Tall and Special Buildings, 12, 83-92, 2003.

[10] Ghojel, J.I., "A New Approach to Modeling Heat Transfer in Compartment Fires," Fire Safety Journal, 31, 227-237, 1998.

[11] Wong, M.B., "Size Effect on Temperatures of Structural Steel in Fire," Journal of Structural Engineering, ASCE, 131 (1), 16-20, 2005.

[12] Gayle, F.W., Fields, R.J., Luecke, W.E., Banovic, S.W., Foecke, T., McGowan, C.N., Siewert, T.A., and McColskey, J.D., "Mechanical and Metallurgical Analysis of Structural 
Steel," NIST NCSTAR 1-3 Federal Building and Fire Safety Investigation of the World Trade Center Disaster, U.S. Department of Commerce, September 2005.

[13] ASTM E408-71(2008), "Standard Test Methods for Total Normal Emittance of Surfaces Using Inspection-Meter Techniques," ASTM Annual Book of Standards, ASTM International, West Conshohocken 2008.

[14] Nelson, K.E., Luedke, E.E., and Bevans, J.T., "A Device for the Rapid Measurement of Total Emittance," Journal of Spacecraft and Rockets, 3 (5), 758-760, 1966.

[15] Hanssen, L.M., and Kaplan, S.G., "Infrared diffuse reflectance instrumentation and standards at NIST," Analytica Chimica Acta, 380, 289-302, 1998.

[16] Hanssen, L.M., Prokhorov, A.V., Khromchenko, V.B., and Mekhontsev, S.N., "Comparison of direct and indirect methods of spectral infrared emittance measurement," in Proc. TEMPMEKO 2004, ed. by D. Zvizdic (LPM/FSB, Zagreb, Croatia, 2005), p. 539-544.

[17] Hanssen, L.M., Cagran, C.P., Prokhorov, A.B., Mekhontsev, S.N., and Khromchenko, V.B., "Use of a High-Temperature Integrating Sphere Reflectometer for Surface-Temperature Measurements," International Journal of Thermophysics, 28 (2), 566-580, 2007.

[18] Cagran, C.P., Hanssen, L.M., Noorma, M., Gura, A.V., and Mekhontsev, S.N., "Temperature-Resolved Infrared Spectral Emissivity of SiC and Pt-10Rh for Temperatures up to $900{ }^{\circ} \mathrm{C}$," International Journal of Thermophysics, 28 (2), 581-597, 2007.

[19] Holman, J.P., Heat Transfer, McGraw-Hill, New York, 1981. 


\section{Appendix A- Raw Data from Fire Test}

While data was obtained during a testing period of $4123 \mathrm{~min}$, only the first $2500 \mathrm{~min}$ of data are tabulated below, as beyond this point, the subsequent changes in temperatures were less than $1^{\circ} \mathrm{F}$. All temperatures are reported in ${ }^{\circ} \mathrm{F}$, as provided by the testing laboratory. It is NIST policy to use SI metric units in all its publications. In this document, however, works of individuals and laboratories outside NIST are cited which report measurements in non-SI units. Datafiles in electronic format are available upon request (dale.bentz@nist.gov).

\subsection{Time and Furnace Temperature Data}

\begin{tabular}{|c|c|c|c|c|c|c|c|c|c|c|c|}
\hline ime & Time & STD & & TC \#2 & & & & & TC \#7 & TC \#8 & AVE \\
\hline$r: \min : \mathrm{sec}$ & Min & TEMP & Deg.F & Deg.F & Deg.F & Deg.F & Deg.F & Deg.F & Deg.F & Deg.F & Deg \\
\hline hannel & $\odot$ & & 1 & 2 & 3 & 4 & 5 & 6 & 7 & 8 & \\
\hline : ๑७ : ๑९ & $\odot$ & 68 & 78 & 74 & 73 & 72 & 75 & 71 & 76 & 73 & 74 \\
\hline $01: 0 \odot$ & 1 & 631 & 104 & 94 & 89 & 87 & 98 & 80 & 102 & 88 & 93 \\
\hline $2: \odot \odot$ & 2 & 799 & 265 & 376 & 222 & 291 & 267 & 209 & 331 & 237 & 275 \\
\hline В:๑९ & 3 & 909 & 636 & 659 & 556 & 683 & 600 & 452 & 772 & 604 & 620 \\
\hline 1:०९ & 4 & 991 & 942 & 946 & 881 & 979 & 875 & 702 & 1072 & 926 & 915 \\
\hline : : $5: \odot \odot$ & 5 & 1055 & 1087 & 1105 & 1044 & 1112 & 1020 & 870 & 1180 & 1072 & 1061 \\
\hline :०6:०९ & 6 & 1108 & 1178 & 1204 & 1132 & 1214 & 1146 & 1021 & 1237 & 1138 & 1159 \\
\hline$\odot \odot$ & 7 & 154 & 1226 & 1270 & 1176 & 1257 & 1214 & 1104 & 281 & 209 & 217 \\
\hline & 8 & 1192 & 1209 & 1264 & 1169 & 1247 & 1207 & 1118 & 244 & 1180 & 205 \\
\hline$\odot \odot$ & 9 & 1226 & 1219 & 1260 & 1172 & 1245 & 1238 & 1156 & 1240 & 1166 & 1212 \\
\hline$\odot \odot$ & 10 & 1257 & 1264 & 1304 & 1192 & 1268 & 1296 & 1205 & 1301 & 1242 & 1259 \\
\hline $0 \odot$ & 11 & 1284 & 1264 & 1295 & 1216 & 1268 & 1288 & 1232 & 1314 & 1303 & 1273 \\
\hline$\odot \odot$ & 12 & 1308 & 1270 & 1302 & 1283 & 1283 & 1300 & 1241 & 1343 & 1273 & 1287 \\
\hline$\odot$ & 13 & 1330 & 1274 & 1314 & 1327 & 1312 & 1321 & 1252 & 1384 & 1271 & 1307 \\
\hline ๑० & 14 & 1350 & 1296 & 1336 & 1382 & 1350 & 1363 & 1294 & 1445 & 1308 & 1347 \\
\hline & 15 & 1369 & 1300 & 1327 & 1407 & 1366 & 1358 & 1315 & 151 & 1366 & 1361 \\
\hline$\odot: 16$ & 16 & 1386 & 1310 & 1304 & 1449 & 1405 & 1387 & 1370 & 1480 & 1416 & 1390 \\
\hline$\odot: 17$ & 17 & 1402 & 1349 & 1367 & 1477 & 1420 & 1485 & 1489 & 1506 & 472 & 1446 \\
\hline 0 & 18 & 1417 & 1375 & 1427 & 1500 & 1425 & 1529 & 1561 & 493 & 474 & 1473 \\
\hline 0 & 19 & 1431 & 1406 & 1437 & 1511 & 1428 & 1532 & 1591 & 1484 & 1473 & 1483 \\
\hline$\odot \odot$ & 20 & 1444 & 1515 & 1468 & 1506 & 1431 & 529 & 1572 & 1462 & 1434 & 1490 \\
\hline $0: 21$ & 21 & 1457 & 1534 & 1478 & 1517 & 1443 & 1518 & 1567 & 1472 & 1435 & 149 \\
\hline $0: 22$ & 22 & 1468 & 1433 & 1439 & 1523 & 1448 & 1455 & 1512 & 1473 & 1437 & 1465 \\
\hline$\odot: 23$ & 23 & 1479 & 1389 & 1433 & 1543 & & & & & 1443 & 1459 \\
\hline ๑० & 24 & & 1394 & 1448 & 1577 & 1512 & 1449 & 1474 & 1511 & 1467 & 1479 \\
\hline & 25 & 1500 & 1415 & 1507 & 1567 & 1518 & 1461 & 1486 & 1528 & 1531 & $15 \odot 2$ \\
\hline$\odot: 2$ & 26 & 1509 & 1430 & 1529 & 1552 & 1559 & 1468 & 1492 & 1533 & 1549 & 1514 \\
\hline$\odot: 27$ & 27 & 1518 & 1444 & 1521 & 1548 & 1557 & 1477 & 1495 & 1541 & 1566 & 1519 \\
\hline$\odot: 28: \odot \odot$ & 28 & 1527 & 1498 & 1519 & 1549 & 1540 & 1486 & 1492 & 1554 & 1603 & 1530 \\
\hline$\odot: 29$ & 29 & 1535 & 1622 & 1529 & 1545 & 1535 & 1497 & 1494 & 569 & 1622 & 1552 \\
\hline & 30 & & 1661 & 1553 & & & & & & & 564 \\
\hline & 31 & & 1676 & 1579 & 1552 & 1562 & 1540 & 1533 & 1559 & 1603 & 1576 \\
\hline$\odot: 32$ & 32 & 1558 & 1676 & 1583 & 1563 & 1566 & 1556 & 1555 & 1557 & 1603 & 1582 \\
\hline$\odot: 33: \odot \odot$ & 33 & 1565 & 1661 & 1588 & 1580 & 1582 & 1564 & 1564 & 1559 & 1617 & 1589 \\
\hline$\odot: 34: \odot \odot$ & 34 & 1571 & 1641 & 1590 & 1590 & 1595 & 1567 & 1569 & 1567 & 1626 & 1593 \\
\hline & 35 & 1578 & 1554 & 1567 & $16 \odot 2$ & 1611 & 1565 & 1576 & 1573 & 1627 & 1584 \\
\hline$\odot: 36: \odot \odot$ & 36 & 1584 & 1533 & 1561 & 1615 & 1618 & 1564 & 1576 & 1578 & 1633 & 1585 \\
\hline
\end{tabular}




\begin{tabular}{|c|c|c|c|c|c|c|c|c|c|c|c|}
\hline$\odot: 37: \odot \odot$ & 37 & 1590 & 1526 & 1562 & 1622 & 1623 & 1566 & 1576 & 1587 & 1638 & 1587 \\
\hline$\odot: 38: \odot \odot$ & 38 & 1596 & 1541 & 1581 & 1631 & 1635 & 1582 & 1595 & 1587 & 1636 & 1599 \\
\hline$\odot: 39: \odot \odot$ & 39 & 1602 & 1551 & 1594 & 1632 & 1635 & 1582 & 1592 & 1596 & 1654 & 1605 \\
\hline$\odot: 40: \odot \odot$ & $4 \odot$ & 1608 & 1605 & 1620 & 1636 & 1633 & 1593 & 1602 & 1605 & 1660 & 1619 \\
\hline : $41: \odot \odot$ & 41 & 1613 & 1637 & 1635 & 1639 & 1635 & 1602 & 1613 & 1606 & 1672 & 1630 \\
\hline$\odot: 42: \odot \odot$ & 42 & 1618 & 1649 & 1655 & 1646 & 1654 & 1614 & 1622 & 1614 & 1673 & 164 \\
\hline$\odot: 43: \odot \odot$ & 43 & 1623 & 1663 & 1663 & 1660 & 1660 & 1623 & 1631 & 1623 & 1682 & 165 \\
\hline$\odot: 44: \odot \odot$ & 44 & 1628 & 1677 & 1682 & 1667 & 1671 & 1640 & 1647 & 1626 & 1676 & $16 t$ \\
\hline$\odot: 45: \odot \odot$ & 45 & 1633 & 1680 & 1682 & 1675 & 1680 & 1648 & 1662 & 1618 & 1669 & 166 \\
\hline$\odot: 46: \odot \odot$ & 46 & 1638 & 1681 & 1678 & 1676 & 1685 & 1644 & 1650 & 1620 & 1667 & 166 \\
\hline$\odot: 47: \odot \odot$ & 47 & 1643 & 1682 & 1677 & 1673 & 1680 & 1640 & 1652 & 1615 & 1664 & 166 \\
\hline$\odot: 48: \odot \odot$ & 48 & 1647 & 1682 & 1684 & 1681 & 1685 & 1647 & 1657 & 1624 & 1667 & 166 \\
\hline$\odot: 49: \odot \odot$ & 49 & 1651 & 1695 & 1686 & 1681 & 1691 & 1648 & 1656 & 1630 & 1674 & 167 \\
\hline$\odot: 5 \odot: \odot \odot$ & 50 & 1656 & 1700 & 1693 & 1690 & 1693 & 1661 & 1668 & 1635 & 1680 & 167 \\
\hline$\odot: 51: \odot \odot$ & 51 & 1660 & 1708 & 1700 & 1690 & 1703 & 1669 & 1676 & 1642 & 1685 & 168 \\
\hline$\odot: 52: \odot \odot$ & 52 & 1664 & 1709 & 1705 & 1696 & 1710 & 1670 & 1685 & 1646 & 1690 & 168 \\
\hline$\odot: 53: \odot \odot$ & 53 & 1668 & 1711 & 1714 & 1698 & 1715 & 1677 & 1691 & 1649 & 1697 & 169 \\
\hline$\odot: 54: \odot \odot$ & 54 & 1672 & 1725 & 1719 & 1705 & 1716 & 1682 & 1697 & 1655 & 1703 & 170 \\
\hline$\odot: 55: \odot \odot$ & 55 & 1676 & 1732 & 1719 & 1709 & 1722 & 1690 & 1711 & 1658 & 1702 & 170 \\
\hline$\odot: 56: \odot \odot$ & 56 & 1680 & 1724 & 1723 & 1712 & 1725 & 1687 & 1704 & 1661 & 1703 & 170 \\
\hline$: \odot \odot$ & 57 & 1684 & 1701 & 1700 & 1703 & 1711 & 1667 & 1685 & 1653 & 1691 & 168 \\
\hline$\odot: 58: \odot \odot$ & 58 & 1687 & 1691 & 1680 & 1675 & 1688 & 1648 & 1663 & 1639 & 1674 & 167 \\
\hline$\odot: 59: \odot \odot$ & 59 & 1691 & 1686 & 1675 & 1674 & 1688 & 1646 & 1669 & 1637 & 1667 & 166 \\
\hline 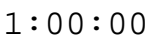 & 60 & 1694 & 1695 & 1686 & 1689 & 1700 & 1663 & 1678 & 1652 & 1683 & 168 \\
\hline 1:०1:०๑ & 61 & 1698 & 1714 & 1718 & 1697 & 1724 & 1685 & 1700 & 1678 & 1693 & 170 \\
\hline 1:०2:๑๑ & 62 & 1701 & 1718 & 1714 & 1719 & 1730 & 1692 & 1706 & 1686 & 1713 & 171 \\
\hline 3:०० & 63 & 1705 & 1730 & 1718 & 1724 & 1734 & 1695 & 1709 & 1690 & 1724 & 171 \\
\hline 1:๑4:๑๑ & 64 & 1708 & 1731 & 1721 & 1734 & 1741 & 1705 & 1723 & 1698 & 1721 & 172 \\
\hline $1: \odot 5: \odot \odot$ & 65 & 1711 & 1735 & 1725 & 1751 & 1746 & 1709 & 1730 & 1702 & 1723 & 172 \\
\hline $1: \odot 6: \odot \odot$ & 66 & 1715 & 1742 & 1732 & 1746 & 1753 & 1720 & 1740 & 1711 & 1727 & 173 \\
\hline 1:०7:๑๑ & 67 & 1718 & 1747 & 1737 & 1754 & 1762 & 1726 & 1747 & 1719 & 1731 & 174 \\
\hline 1:๑৪:๑९ & 68 & 1721 & 1751 & 1740 & 1754 & 1765 & 1731 & 1755 & 1726 & 1735 & 174 \\
\hline $9: \odot \odot$ & 69 & 1724 & 1735 & 1738 & 1748 & 1763 & 1722 & 1753 & 1723 & 1727 & 173 \\
\hline $1: 10: \odot \odot$ & 70 & 1727 & 1732 & 1730 & 1736 & 1754 & 1711 & 1734 & 1714 & 1721 & 172 \\
\hline $1: 11: \odot \odot$ & 71 & 1730 & 1741 & 1729 & 1735 & 1749 & 1715 & 1736 & 1714 & 1720 & 173 \\
\hline $1: 12: \odot \odot$ & 72 & 1733 & 1750 & 1732 & 1731 & 1751 & 1720 & 1741 & 1718 & 1726 & 173 \\
\hline $1: 13: \odot \odot$ & 73 & 1736 & 1758 & 1738 & 1740 & 1756 & 1725 & 1744 & 1718 & 1726 & 173 \\
\hline $1: 14: \odot \odot$ & 74 & 1739 & 1762 & 1743 & 1741 & 1756 & 1721 & 1741 & 1721 & 1729 & 173 \\
\hline$: \odot \odot$ & 75 & 1742 & 1761 & 1747 & 1743 & 1757 & 1723 & 1745 & 1722 & 1730 & 174 \\
\hline $1: 16: \odot \odot$ & 76 & & 1591 & 1598 & 1606 & 1592 & 1576 & 1592 & 1584 & 1577 & 159 \\
\hline $1: 17: \odot \odot$ & 77 & & 1410 & 1401 & 1412 & 1389 & 1388 & 1398 & 1397 & 1389 & 139 \\
\hline $1: 18: \odot \odot$ & 78 & & 1303 & 1289 & 1303 & 1275 & 1284 & 1286 & 1289 & 1282 & 128 \\
\hline $1: 19: \odot \odot$ & 79 & & 1234 & 1218 & 1235 & 1205 & 1218 & 1212 & 1226 & 1210 & 122 \\
\hline $1: 2 \odot: \odot \odot$ & 80 & & 1183 & 1164 & 1183 & 1151 & 1168 & 11 & 1180 & 1156 & 116 \\
\hline $1: 21: \odot \odot$ & 81 & & 1141 & 1118 & 1141 & 1105 & 1125 & & 1140 & 1111 & 112 \\
\hline $1: 22: \odot \odot$ & 82 & & 1110 & 1082 & 1106 & 1069 & 1092 & 1067 & 1107 & 1079 & 108 \\
\hline $1: 23: \odot \odot$ & 83 & & 1084 & 1051 & 1076 & 1036 & 1063 & 1035 & 1079 & 1051 & 105 \\
\hline $1: 24: \odot \odot$ & 84 & & 1057 & 1024 & 1048 & 1006 & 1036 & 1008 & 1054 & 1025 & 103 \\
\hline $1: 25: \odot \odot$ & 85 & & 1036 & 1002 & 1025 & 981 & 1015 & 987 & 1036 & 1001 & 101 \\
\hline $1: 26: \odot \odot$ & 86 & & 1016 & 979 & 1002 & 957 & 992 & 964 & 1016 & 980 & 988 \\
\hline $1: 27: \odot \odot$ & 87 & & 996 & 959 & 981 & 936 & 969 & 944 & 997 & 960 & 968 \\
\hline $1: 28: \odot \odot$ & 88 & & 976 & 940 & 963 & 917 & 947 & 922 & 978 & 942 & 948 \\
\hline $1: 29: \odot \odot$ & 89 & & 958 & 921 & 945 & 898 & 929 & 904 & 960 & 924 & 930 \\
\hline $1: 3 \odot: \odot \odot$ & 90 & & 941 & $9 \odot 4$ & 927 & 880 & 913 & 886 & 944 & 907 & 913 \\
\hline $1: 31: \odot \odot$ & 91 & & 925 & 886 & 909 & 862 & 897 & 869 & 927 & 890 & 896 \\
\hline $1: 32: \odot \odot$ & 92 & & 911 & 870 & 893 & 846 & 881 & 854 & 912 & 875 & 880 \\
\hline $1: 33: \odot \odot$ & 93 & & 896 & 855 & 879 & 830 & 867 & 838 & 898 & 860 & 865 \\
\hline
\end{tabular}




\begin{tabular}{|c|c|c|c|c|c|c|c|c|c|c|}
\hline $1: 34: \odot \odot$ & 94 & 881 & 839 & 863 & 814 & 852 & 823 & 884 & 845 & 850 \\
\hline $1: 35: \odot \odot$ & 95 & 869 & 826 & 851 & 799 & 839 & 810 & 871 & 833 & 837 \\
\hline $1: 36: \odot \odot$ & 96 & 855 & 811 & 836 & 783 & 825 & 796 & 857 & 818 & 823 \\
\hline $1: 37: \odot \odot$ & 97 & 843 & 797 & 821 & 770 & 812 & 783 & 842 & 803 & 809 \\
\hline 1:38:०९ & 98 & 831 & 783 & 809 & 756 & 799 & 770 & 828 & 790 & 796 \\
\hline $1: 39: \odot \odot$ & 99 & 819 & 769 & 794 & 743 & 787 & 758 & 813 & 775 & 782 \\
\hline $1: 4 \odot: \odot \odot$ & 100 & 806 & 755 & 780 & 729 & 776 & 746 & 799 & 760 & 769 \\
\hline $1: 41: \odot \odot$ & 101 & 796 & 744 & 767 & 716 & 766 & 736 & 788 & 748 & 758 \\
\hline $1: 42: \odot \odot$ & 102 & 785 & 731 & 754 & 703 & 754 & 726 & 777 & 735 & 746 \\
\hline $1: 43: \odot \odot$ & 103 & 774 & 719 & 742 & 692 & 743 & 713 & 765 & 724 & 734 \\
\hline $1: 44: \odot \odot$ & 104 & 763 & 708 & 730 & 682 & 731 & 703 & 755 & 712 & 723 \\
\hline $1: 45: \odot \odot$ & 105 & 751 & 697 & 719 & 670 & 719 & 693 & 743 & 700 & 711 \\
\hline $1: 46: \odot \odot$ & 106 & 741 & 686 & 708 & 660 & 709 & 682 & 732 & 689 & 701 \\
\hline $1: 47: \odot \odot$ & 107 & 731 & 677 & 699 & 650 & 697 & 671 & 722 & 679 & 691 \\
\hline $1: 48: \odot \odot$ & 108 & 721 & 667 & 690 & 640 & 687 & 661 & 713 & 669 & 681 \\
\hline $1: 49: \odot \odot$ & 109 & 713 & 658 & 680 & 631 & 678 & 652 & 704 & 659 & 672 \\
\hline 1:5०:०๑ & 110 & 704 & 650 & 671 & 622 & 668 & 642 & 695 & 651 & 663 \\
\hline $1: 51: \odot \odot$ & 111 & 695 & 641 & 661 & 612 & 659 & 632 & 687 & 641 & 654 \\
\hline $1: 52: \odot \odot$ & 112 & 687 & 633 & 653 & 604 & 651 & 623 & 679 & 633 & 645 \\
\hline $1: 53: \odot \odot$ & 113 & 678 & 624 & 644 & 595 & 641 & 614 & 670 & 623 & 636 \\
\hline $1: 54: \odot \odot$ & 114 & 669 & 616 & 636 & 586 & 633 & 606 & 661 & 615 & 628 \\
\hline $1: 55: \odot \odot$ & 115 & 661 & 608 & 627 & 579 & 627 & 600 & 652 & 605 & 620 \\
\hline $1: 56: \odot \odot$ & 116 & 653 & 600 & 618 & 570 & 619 & 593 & 643 & 597 & 612 \\
\hline $1: 57: \odot \odot$ & 117 & 644 & 592 & 610 & 562 & 610 & 584 & 636 & 588 & 603 \\
\hline $1: 58: \odot \odot$ & 118 & 636 & 585 & 602 & 555 & 602 & 577 & 628 & 580 & 596 \\
\hline $1: 59: \odot \odot$ & 119 & 628 & 577 & 595 & 549 & 595 & 571 & 619 & 572 & 588 \\
\hline $2: \odot \odot: \odot \odot$ & 120 & 621 & 570 & 587 & 542 & 588 & 564 & 611 & 564 & 581 \\
\hline $2: \odot 1: \odot \odot$ & 121 & 614 & 563 & 580 & 536 & 581 & 557 & 604 & 557 & 574 \\
\hline $2: 02: \odot \odot$ & 122 & 606 & 555 & 573 & 529 & 573 & 550 & 596 & 550 & 566 \\
\hline $2: \odot 3: \odot \odot$ & 123 & $6 \odot \odot$ & 550 & 567 & 523 & 567 & 545 & 591 & 544 & 561 \\
\hline $2: \odot 4: \odot \odot$ & 124 & 593 & 542 & 560 & 516 & 560 & 538 & 583 & 535 & 554 \\
\hline $2: \odot 5: \odot \odot$ & 125 & 586 & 535 & 553 & 508 & 554 & 530 & 577 & 528 & 546 \\
\hline $2: \odot 6: \odot \odot$ & 126 & 579 & 528 & 546 & 502 & 550 & 525 & 569 & 522 & 540 \\
\hline $2: \odot 7: \odot \odot$ & 127 & 572 & 522 & 539 & 495 & 543 & 519 & 563 & 515 & 534 \\
\hline 2:๑8:๑९ & 128 & 566 & 516 & 535 & 489 & 537 & 513 & 557 & 509 & 528 \\
\hline $2: \odot 9: \odot \odot$ & 129 & $56 \odot$ & 510 & 529 & 485 & 531 & 507 & 550 & 503 & 522 \\
\hline $2: 10: \odot \odot$ & 130 & 554 & 505 & 523 & 479 & 527 & 502 & 545 & 497 & 516 \\
\hline $2: 11: \odot \odot$ & 131 & 548 & 499 & 517 & 473 & 521 & 495 & 538 & 492 & 510 \\
\hline $2: 12: \odot \odot$ & 132 & 543 & 495 & 512 & 469 & 516 & 491 & 533 & 487 & 506 \\
\hline $2: 13: \odot \odot$ & 133 & 536 & 488 & 505 & 462 & 511 & 485 & 526 & 481 & 499 \\
\hline $2: 14: \odot \odot$ & 134 & 530 & 483 & 499 & 456 & 506 & 480 & 522 & 475 & 494 \\
\hline $2: 15: \odot \odot$ & 135 & 526 & 479 & 495 & 452 & 501 & 476 & 517 & 470 & 490 \\
\hline $2: 16: \odot \odot$ & 136 & 520 & 474 & 490 & 446 & 497 & 472 & 512 & 466 & 485 \\
\hline $2: 17: \odot \odot$ & 137 & 516 & 470 & 484 & 442 & 493 & 466 & 509 & 461 & 480 \\
\hline $2: 18: \odot \odot$ & 138 & 510 & 465 & 481 & 438 & 487 & 461 & 503 & 456 & 475 \\
\hline $2: 19: \odot \odot$ & 139 & 505 & 460 & 476 & 434 & 482 & 456 & 497 & 451 & 470 \\
\hline $2: 2 \odot: \odot \odot$ & 140 & 498 & 456 & 471 & 429 & 477 & 450 & 492 & 445 & 465 \\
\hline $2: 21: \odot \odot$ & 141 & 494 & 452 & 467 & 425 & 472 & 446 & 487 & 441 & 460 \\
\hline $2: 22: \odot \odot$ & 142 & 489 & 447 & 463 & 421 & 467 & 441 & 482 & 437 & 456 \\
\hline $2: 23: \odot \odot$ & 143 & 485 & 443 & 459 & 417 & 463 & 436 & 478 & 433 & 452 \\
\hline $2: 24: \odot \odot$ & 144 & 480 & 439 & 455 & 414 & 460 & 433 & 474 & 430 & 448 \\
\hline $2: 25: \odot \odot$ & 145 & 474 & 434 & 451 & 409 & 456 & 429 & 469 & 426 & 444 \\
\hline $2: 26: \odot \odot$ & 146 & 467 & 430 & 447 & 404 & 450 & 424 & 464 & 420 & 438 \\
\hline $2: 27: \odot \odot$ & 147 & 461 & 426 & 443 & 400 & 446 & 419 & 460 & 416 & 434 \\
\hline $2: 28: \odot \odot$ & 148 & 456 & 422 & 439 & 397 & 442 & 416 & 454 & 412 & 430 \\
\hline $2: 29: \odot \odot$ & 149 & 449 & 418 & 435 & 392 & 438 & 412 & 450 & 408 & 425 \\
\hline $2: 3 \odot: \odot \odot$ & 150 & 445 & 415 & 431 & 389 & 435 & 408 & 445 & 403 & 421 \\
\hline
\end{tabular}




\begin{tabular}{|c|c|c|c|c|c|c|c|c|c|c|}
\hline $2: 31: \odot \odot$ & 151 & 442 & 411 & 428 & 386 & 431 & 404 & 442 & 399 & 418 \\
\hline $2: 32: \odot \odot$ & 152 & 439 & 408 & 425 & 383 & 429 & 401 & 438 & 397 & 415 \\
\hline $2: 33: \odot \odot$ & 153 & 437 & 406 & 422 & 379 & 426 & 397 & 435 & 393 & 412 \\
\hline $2: 34: \odot \odot$ & 154 & 433 & 403 & 419 & 376 & 424 & 393 & 432 & 390 & $4 \odot 9$ \\
\hline $2: 35: \odot \odot$ & 155 & 431 & 401 & 415 & 373 & 421 & 391 & 429 & 387 & 406 \\
\hline $2: 36: \odot \odot$ & 156 & 428 & 398 & 411 & 370 & 419 & 389 & 426 & 385 & 403 \\
\hline $2: 37: \odot \odot$ & 157 & 426 & 394 & 408 & 367 & 416 & 386 & 423 & 381 & $40 \odot$ \\
\hline $2: 38: \odot \odot$ & 158 & 423 & 392 & 405 & 364 & 414 & 384 & 419 & 377 & 397 \\
\hline $2: 39: \odot \odot$ & 159 & 419 & 388 & 401 & 362 & 410 & 381 & 416 & 375 & 394 \\
\hline $2: 4 \odot: \odot \odot$ & 160 & 414 & 385 & 399 & 358 & 406 & 377 & 412 & 371 & 390 \\
\hline $2: 41: \odot \odot$ & 161 & 407 & 381 & 395 & 356 & 402 & 375 & 408 & 367 & 386 \\
\hline $2: 42: \odot \odot$ & 162 & 403 & 378 & 393 & 353 & 399 & 371 & 404 & 364 & 383 \\
\hline $2: 43: \odot \odot$ & 163 & 398 & 374 & 389 & 350 & 396 & 368 & $40 \odot$ & 361 & 379 \\
\hline $2: 44: \odot \odot$ & 164 & 393 & 371 & 385 & 347 & 393 & 364 & 398 & 358 & 376 \\
\hline $2: 45: \odot \odot$ & 165 & 389 & 369 & 382 & 346 & 390 & 362 & 394 & 356 & 373 \\
\hline $2: 46: \odot \odot$ & 166 & 385 & 366 & 380 & 343 & 387 & 359 & 391 & 353 & 371 \\
\hline $2: 47: \odot \odot$ & 167 & 383 & 365 & 377 & 340 & 385 & 357 & 390 & 350 & 368 \\
\hline $2: 48: \odot \odot$ & 168 & 381 & 363 & 375 & 338 & 383 & 354 & 387 & 348 & 366 \\
\hline $2: 49: \odot \odot$ & 169 & 380 & 360 & 372 & 337 & 380 & 353 & 384 & 346 & 364 \\
\hline $2: 5 \odot: \odot \odot$ & 170 & 378 & 357 & 370 & 334 & 377 & 351 & 382 & 343 & 361 \\
\hline $2: 51: \odot \odot$ & 171 & 377 & 355 & 368 & 332 & 376 & 348 & 379 & 341 & 360 \\
\hline $2: 52: \odot \odot$ & 172 & 374 & 353 & 366 & 329 & 374 & 347 & 378 & 338 & 357 \\
\hline $2: 53: \odot \odot$ & 173 & 373 & 350 & 363 & 327 & 372 & 345 & 374 & 336 & 355 \\
\hline $2: 54: \odot \odot$ & 174 & 371 & 348 & 361 & 325 & 370 & 343 & 372 & 335 & 353 \\
\hline $2: 55: \odot \odot$ & 175 & 368 & 346 & 359 & 323 & 367 & 340 & 370 & 332 & 351 \\
\hline $2: 56: \odot \odot$ & 176 & 366 & 343 & 356 & 321 & 365 & 339 & 369 & 330 & 349 \\
\hline $2: 57: \odot \odot$ & 177 & 364 & 341 & 355 & 318 & 363 & 337 & 366 & 328 & 347 \\
\hline $2: 58: \odot \odot$ & 178 & 362 & 338 & 353 & 317 & 360 & 335 & 364 & 325 & 344 \\
\hline $2: 59: \odot \odot$ & 179 & 361 & 337 & 350 & 315 & 357 & 332 & 363 & 323 & 342 \\
\hline $3: \odot \odot: \odot \odot$ & 180 & 358 & 335 & 348 & 313 & 356 & 331 & 361 & 322 & 341 \\
\hline $3: 01: \odot \odot$ & 181 & 356 & 333 & 347 & 311 & 355 & 330 & 359 & 319 & 339 \\
\hline $3: \odot 2: \odot \odot$ & 182 & 354 & 332 & 345 & 310 & 352 & 327 & 357 & 318 & 337 \\
\hline $3: \odot 3: \odot \odot$ & 183 & 350 & 330 & 344 & 308 & 349 & 325 & 355 & 316 & 335 \\
\hline $3: \odot 4: \odot \odot$ & 184 & 347 & 327 & 341 & 305 & 347 & 322 & 354 & 313 & 332 \\
\hline $3: \odot 5: \odot \odot$ & 185 & 345 & 325 & 339 & 303 & 344 & 321 & 351 & 311 & 330 \\
\hline $3: \odot 6: \odot \odot$ & 186 & 342 & 323 & 337 & 301 & 342 & 319 & 349 & 308 & 327 \\
\hline $3: \odot 7: \odot \odot$ & 187 & 339 & 322 & 334 & 298 & 341 & 316 & 348 & 307 & 326 \\
\hline $3: \odot 8: \odot \odot$ & 188 & 336 & 321 & 332 & 296 & 339 & 314 & 345 & 305 & 324 \\
\hline $3: \odot 9: \odot \odot$ & 189 & 333 & 318 & 330 & 293 & 337 & 313 & 343 & 304 & 321 \\
\hline $3: 10: \odot \odot$ & 190 & 331 & 317 & 328 & 292 & 335 & 310 & 341 & 301 & 319 \\
\hline $3: 11: \odot \odot$ & 191 & 329 & 316 & 325 & 289 & 334 & 308 & 339 & 300 & 317 \\
\hline $3: 12: \odot \odot$ & 192 & 326 & 315 & 324 & 288 & 332 & 307 & 338 & 298 & 316 \\
\hline $3: 13: \odot \odot$ & 193 & 324 & 313 & 321 & 286 & 330 & 304 & 337 & 297 & 314 \\
\hline $3: 14: \odot \odot$ & 194 & 321 & 311 & 320 & 283 & 328 & 302 & 334 & 296 & 312 \\
\hline $3: 15: \odot \odot$ & 195 & 320 & 310 & 318 & 282 & 327 & 301 & 333 & 293 & 311 \\
\hline $3: 16: \odot \odot$ & 196 & 319 & 308 & 317 & 280 & 325 & 298 & 330 & 292 & 309 \\
\hline $3: 17: \odot \odot$ & 197 & 317 & 306 & 315 & 277 & 323 & 297 & 329 & 291 & 307 \\
\hline $3: 18: \odot \odot$ & 198 & 314 & 305 & 313 & 276 & 322 & 295 & 328 & 290 & 305 \\
\hline $3: 19: \odot \odot$ & 199 & 313 & 304 & 311 & 274 & 320 & 294 & 326 & 289 & 304 \\
\hline $3: 2 \odot: \odot \odot$ & 200 & 311 & 302 & 310 & 273 & 319 & 292 & 324 & 288 & 302 \\
\hline $3: 21: \odot \odot$ & 201 & 310 & 301 & 309 & 271 & 317 & 290 & 323 & 286 & 301 \\
\hline $3: 22: \odot \odot$ & 202 & 308 & 299 & 308 & 270 & 315 & 289 & 321 & 284 & 299 \\
\hline $3: 23: \odot \odot$ & 203 & 306 & 297 & 307 & 269 & 314 & 288 & 320 & 283 & 298 \\
\hline $3: 24: \odot \odot$ & 204 & 305 & 296 & 304 & 267 & 312 & 287 & 318 & 282 & 296 \\
\hline $3: 25: \odot \odot$ & 205 & 303 & 295 & 303 & 266 & 311 & 286 & 317 & 280 & 295 \\
\hline $3: 26: \odot \odot$ & 206 & 300 & 294 & 301 & 265 & 308 & 283 & 316 & 278 & 293 \\
\hline $3: 27: \odot \odot$ & 207 & 299 & 291 & 299 & 263 & 307 & 282 & 314 & 277 & 292 \\
\hline
\end{tabular}




\begin{tabular}{|c|c|c|c|c|c|c|c|c|c|c|}
\hline $3: 28: \odot \odot$ & 208 & 297 & 290 & 298 & 262 & 306 & 281 & 313 & 276 & 290 \\
\hline $3: 29: 00$ & 209 & 296 & 289 & 297 & 260 & 305 & 280 & 311 & 274 & 289 \\
\hline $3: 30: \odot \odot$ & 210 & 293 & 288 & 295 & 259 & 303 & 279 & 309 & 273 & 287 \\
\hline $3: 31: \odot \odot$ & 211 & 292 & 287 & 294 & 258 & 301 & 276 & 308 & 271 & 286 \\
\hline $3: 32: \odot \odot$ & 212 & 290 & 285 & 292 & 257 & 299 & 275 & 306 & 270 & 284 \\
\hline $3: 33: \odot \odot$ & 213 & 289 & 283 & 291 & 255 & 298 & 275 & 304 & 270 & 283 \\
\hline $3: 34: \odot \odot$ & 214 & 288 & 282 & 289 & 254 & 297 & 273 & 303 & 267 & 282 \\
\hline $3: 35: \odot \odot$ & 215 & 287 & 281 & 288 & 253 & 296 & 272 & 302 & 266 & 280 \\
\hline $3: 36: \odot \odot$ & 216 & 285 & 280 & 288 & 252 & 295 & 270 & 299 & 265 & 279 \\
\hline $3: 37: \odot \odot$ & 217 & 284 & 279 & 287 & 251 & 294 & 269 & 298 & 264 & 278 \\
\hline $3: 38: \odot \odot$ & 218 & 283 & 278 & 284 & 249 & 292 & 269 & 297 & 263 & 277 \\
\hline $3: 39: \odot \odot$ & 219 & 281 & 276 & 283 & 248 & 291 & 268 & 296 & 262 & 276 \\
\hline $3: 4 \odot: \odot \odot$ & 220 & 280 & 275 & 282 & 246 & 290 & 267 & 295 & 260 & 274 \\
\hline $3: 41: 0 \odot$ & 221 & 279 & 273 & 281 & 245 & 289 & 265 & 294 & 259 & 273 \\
\hline $3: 42: \odot \odot$ & 222 & 279 & 272 & 280 & 244 & 287 & 263 & 293 & 258 & 272 \\
\hline $3: 43: \odot \odot$ & 223 & 279 & 270 & 277 & 243 & 286 & 262 & 291 & 257 & 271 \\
\hline $3: 44: \odot \odot$ & 224 & 279 & 269 & 276 & 242 & 284 & 260 & 290 & 256 & 269 \\
\hline $3: 45: \odot \odot$ & 225 & 278 & 267 & 276 & 240 & 283 & 258 & 289 & 255 & 268 \\
\hline $3: 46: \odot \odot$ & 226 & 276 & 267 & 274 & 239 & 281 & 258 & 288 & 254 & 267 \\
\hline $3: 47: \odot \odot$ & 227 & 275 & 266 & 273 & 239 & 280 & 257 & 287 & 253 & 266 \\
\hline $3: 48: \odot \odot$ & 228 & 273 & 265 & 272 & 238 & 279 & 256 & 286 & 251 & 265 \\
\hline $3: 49: \odot \odot$ & 229 & 272 & 264 & 271 & 238 & 278 & 256 & 285 & 250 & 264 \\
\hline $3: 5 \odot: \odot \odot$ & 230 & 271 & 264 & 271 & 237 & 278 & 255 & 285 & 249 & 264 \\
\hline $3: 51: \odot \odot$ & 231 & 269 & 262 & $27 \odot$ & 236 & 277 & 254 & 283 & 248 & 262 \\
\hline $3: 52: \odot \odot$ & 232 & 267 & 262 & 269 & 236 & 276 & 252 & 282 & 248 & 262 \\
\hline $3: 53: \odot \odot$ & 233 & 267 & 261 & 267 & 235 & 276 & 251 & 281 & 247 & 261 \\
\hline $3: 54: \odot \odot$ & 234 & 266 & 260 & 266 & 235 & 275 & 251 & 281 & 246 & 260 \\
\hline $3: 55: \odot \odot$ & 235 & 265 & 259 & 265 & 234 & 274 & 250 & 280 & 245 & 259 \\
\hline $3: 56: \odot \odot$ & 236 & 264 & 259 & 265 & 234 & 274 & 249 & 279 & 244 & 258 \\
\hline $3: 57: \odot \odot$ & 237 & 262 & 258 & 264 & 233 & 272 & 249 & 278 & 242 & 257 \\
\hline $3: 58: \odot \odot$ & 238 & 260 & 256 & 262 & 232 & 272 & 248 & 277 & 242 & 256 \\
\hline $3: 59: \odot \odot$ & 239 & 259 & 255 & 261 & 231 & 271 & 247 & 276 & 241 & 255 \\
\hline 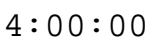 & 240 & 258 & 254 & 260 & 229 & 269 & 246 & 275 & 240 & 254 \\
\hline 4:०1:०९ & 241 & 258 & 253 & 259 & 227 & 269 & 245 & 274 & 239 & 253 \\
\hline $4: \odot 2: \odot \odot$ & 242 & 258 & 253 & 258 & 227 & 268 & 244 & 274 & 239 & 252 \\
\hline 4:०3:๑९ & 243 & 259 & 252 & 256 & 225 & 266 & 242 & 272 & 238 & 251 \\
\hline $4: \odot 4: \odot \odot$ & 244 & 260 & 251 & 256 & 224 & 265 & 241 & 271 & 236 & 251 \\
\hline $4: \odot 5: \odot \odot$ & 245 & 260 & 250 & 255 & 224 & 264 & 240 & 270 & 236 & 250 \\
\hline $4: \odot 6: \odot \odot$ & 246 & 260 & 248 & 254 & 223 & 263 & 239 & 269 & 235 & 249 \\
\hline $4: \odot 7: \odot \odot$ & 247 & 260 & 248 & 254 & 222 & 262 & 238 & 268 & 235 & 248 \\
\hline 4:०৪:๑९ & 248 & $26 \odot$ & 247 & 253 & 221 & 260 & 236 & 267 & 234 & 247 \\
\hline 4:०९:๑९ & 249 & 260 & 246 & 252 & 220 & 259 & 235 & 266 & 234 & 247 \\
\hline $4: 10: \odot \odot$ & 250 & 260 & 245 & 251 & 218 & 258 & 234 & 265 & 233 & 246 \\
\hline $4: 11: \odot \odot$ & 251 & $26 \odot$ & 245 & $25 \odot$ & 217 & 257 & 233 & 264 & 232 & 245 \\
\hline $4: 12: \odot \odot$ & 252 & 260 & 244 & 250 & 217 & 257 & 233 & 264 & 231 & 244 \\
\hline $4: 13: \odot \odot$ & 253 & 260 & 243 & 249 & 216 & 256 & 231 & 263 & 229 & 243 \\
\hline $4: 14: \odot \odot$ & 254 & 260 & 242 & 248 & 215 & 255 & 231 & 262 & 228 & 243 \\
\hline $4: 15: \odot \odot$ & 255 & $26 \odot$ & 240 & 248 & 214 & 254 & 230 & 261 & 228 & 242 \\
\hline $4: 16: \odot \odot$ & 256 & 260 & 239 & 247 & 214 & 254 & 229 & 260 & 227 & 241 \\
\hline $4: 17: \odot \odot$ & 257 & 259 & 239 & 245 & 213 & 253 & 228 & 260 & 226 & 240 \\
\hline $4: 18: \odot \odot$ & 258 & 259 & 238 & 245 & 213 & 251 & 227 & 258 & 226 & 240 \\
\hline $4: 19: \odot \odot$ & 259 & 259 & 237 & 244 & 212 & 251 & 227 & 257 & 225 & 239 \\
\hline $4: 20: \odot \odot$ & 260 & 258 & 236 & 243 & 211 & 250 & 226 & 257 & 224 & 238 \\
\hline $4: 21: \odot \odot$ & 261 & 258 & 235 & 242 & 210 & 249 & 226 & 256 & 224 & 237 \\
\hline $4: 22: \odot \odot$ & 262 & 257 & 234 & 242 & 210 & 248 & 224 & 255 & 223 & 237 \\
\hline $4: 23: \odot \odot$ & 263 & 257 & 234 & 241 & 209 & 248 & 223 & 254 & 222 & 236 \\
\hline $4: 24: \odot \odot$ & 264 & 256 & 233 & 240 & 209 & 247 & 223 & 253 & 222 & 235 \\
\hline
\end{tabular}




\begin{tabular}{|c|c|c|c|c|c|c|c|c|c|c|}
\hline $4: 25: \odot \odot$ & 265 & 256 & 232 & 240 & 208 & 246 & 222 & 253 & 221 & 235 \\
\hline $4: 26: \odot \odot$ & 266 & 255 & 231 & 239 & 206 & 244 & 221 & 252 & 219 & 233 \\
\hline $4: 27: \odot \odot$ & 267 & 254 & 231 & 238 & 206 & 244 & 221 & 252 & 219 & 233 \\
\hline $4: 28: \odot \odot$ & 268 & 254 & 229 & 238 & 205 & 243 & 220 & 251 & 218 & 232 \\
\hline $4: 29: \odot \odot$ & 269 & 252 & 228 & 237 & 205 & 243 & 220 & 250 & 218 & 232 \\
\hline $4: 3 \odot: \odot \odot$ & 270 & 252 & 228 & 236 & 204 & 242 & 219 & 250 & 217 & 231 \\
\hline $4: 31: \odot \odot$ & 271 & 251 & 227 & 235 & 203 & 242 & 218 & 249 & 216 & 230 \\
\hline $4: 32: \odot \odot$ & 272 & 251 & 227 & 235 & 203 & 241 & 218 & 249 & 215 & 230 \\
\hline $4: 33: \odot \odot$ & 273 & 250 & 226 & 233 & 202 & 240 & 217 & 247 & 215 & 229 \\
\hline $4: 34: \odot \odot$ & 274 & 250 & 225 & 232 & 202 & 239 & 215 & 246 & 214 & 228 \\
\hline $4: 35: \odot \odot$ & 275 & 249 & 225 & 232 & 201 & 239 & 215 & 246 & 213 & 228 \\
\hline $4: 36: \odot \odot$ & 276 & 248 & 224 & 231 & 201 & 238 & 214 & 245 & 213 & 227 \\
\hline $4: 37: \odot \odot$ & 277 & 248 & 224 & 231 & 200 & 238 & 214 & 245 & 212 & 226 \\
\hline $4: 38: \odot \odot$ & 278 & 248 & 223 & 230 & 200 & 236 & 213 & 245 & 212 & 226 \\
\hline $4: 39: \odot \odot$ & 279 & 247 & 223 & 230 & 199 & 236 & 213 & 244 & 211 & 225 \\
\hline $4: 4 \odot: \odot \odot$ & 280 & 247 & 222 & 229 & 199 & 235 & 213 & 244 & 211 & 225 \\
\hline $4: 41: \odot \odot$ & 281 & 246 & 222 & 229 & 199 & 235 & 213 & 244 & 211 & 225 \\
\hline $4: 42: \odot \odot$ & 282 & 245 & 221 & 229 & 199 & 235 & 213 & 243 & 211 & 224 \\
\hline $4: 43: \odot \odot$ & 283 & 243 & 221 & 229 & 199 & 235 & 212 & 243 & 211 & 224 \\
\hline $4: 44: \odot \odot$ & 284 & 241 & 221 & 229 & 199 & 235 & 212 & 243 & 210 & 224 \\
\hline $4: 45: \odot \odot$ & 285 & 240 & 220 & 228 & 199 & 234 & 212 & 242 & 210 & 223 \\
\hline $4: 46: \odot \odot$ & 286 & 239 & 220 & 228 & 199 & 234 & 212 & 242 & 209 & 223 \\
\hline $4: 47: \odot \odot$ & 287 & 239 & 220 & 228 & 199 & 234 & 211 & 241 & 209 & 222 \\
\hline $4: 48: \odot \odot$ & 288 & 239 & 220 & 227 & 199 & 234 & 211 & 241 & 209 & 222 \\
\hline $4: 49: \odot \odot$ & 289 & 239 & 218 & 227 & 199 & 233 & 211 & 241 & 208 & 222 \\
\hline $4: 5 \odot: \odot \odot$ & 290 & 238 & 218 & 227 & 198 & 233 & 211 & 240 & 208 & 222 \\
\hline $4: 51: \odot \odot$ & 291 & 238 & 218 & 227 & 198 & 232 & 211 & 240 & 208 & 221 \\
\hline $4: 52: \odot \odot$ & 292 & 238 & 217 & 226 & 198 & 232 & 210 & 238 & 208 & 221 \\
\hline $4: 53: \odot \odot$ & 293 & 238 & 216 & 226 & 198 & 232 & 210 & 238 & 206 & 221 \\
\hline $4: 54: \odot \odot$ & 294 & 238 & 216 & 226 & 198 & 231 & 210 & 238 & 206 & 220 \\
\hline $4: 55: \odot \odot$ & 295 & 237 & 216 & 225 & 198 & 231 & 210 & 237 & 205 & 220 \\
\hline $4: 56: \odot \odot$ & 296 & 237 & 216 & 225 & 198 & 231 & 209 & 237 & 205 & 220 \\
\hline $4: 57: \odot \odot$ & 297 & 236 & 215 & 225 & 198 & 231 & 209 & 237 & 205 & 219 \\
\hline $4: 58: \odot \odot$ & 298 & 236 & 215 & 224 & 197 & 230 & 209 & 236 & 204 & 219 \\
\hline $4: 59: \odot \odot$ & 299 & 236 & 214 & 224 & 197 & 230 & 209 & 236 & 204 & 219 \\
\hline 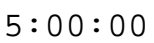 & 300 & 235 & 214 & 224 & 197 & 230 & 209 & 236 & 204 & 219 \\
\hline $5: \odot 1: \odot \odot$ & 301 & 235 & 214 & 222 & 197 & 229 & 208 & 235 & 203 & 218 \\
\hline $5: \odot 2: \odot \odot$ & 302 & 235 & 213 & 222 & 196 & 229 & 208 & 235 & 203 & 218 \\
\hline $5: \odot 3: \odot \odot$ & 303 & 233 & 213 & 222 & 196 & 229 & 207 & 235 & 202 & 217 \\
\hline $5: \odot 4: \odot \odot$ & 304 & 233 & 213 & 221 & 196 & 229 & 207 & 234 & 202 & 217 \\
\hline $5: \odot 5: \odot \odot$ & 305 & 233 & 212 & 221 & 195 & 228 & 206 & 234 & 201 & 216 \\
\hline $5: \odot 6: \odot \odot$ & 306 & 232 & 211 & 220 & 193 & 228 & 204 & 233 & 201 & 215 \\
\hline $5: \odot 7: \odot \odot$ & 307 & 232 & 211 & 220 & 192 & 227 & 204 & 233 & 201 & 215 \\
\hline 5:०৪:๑९ & 308 & 231 & 210 & 219 & 192 & 226 & 203 & 232 & 200 & 214 \\
\hline 5:๑9:๑९ & 309 & 231 & 209 & 218 & 191 & 224 & 202 & 231 & 199 & 213 \\
\hline $5: 10: \odot \odot$ & 310 & 230 & 208 & 217 & 190 & 224 & 202 & 230 & 199 & 212 \\
\hline $5: 11: \odot \odot$ & 311 & 230 & 207 & 216 & 190 & 223 & 201 & 230 & 198 & 212 \\
\hline $5: 12: \odot \odot$ & 312 & 229 & 206 & 216 & 189 & 223 & 201 & 229 & 197 & 211 \\
\hline $5: 13: \odot \odot$ & 313 & 229 & 206 & 215 & 189 & 222 & 200 & 227 & 195 & 210 \\
\hline $5: 14: \odot \odot$ & 314 & 228 & 204 & 215 & 188 & 221 & 199 & 227 & 195 & 210 \\
\hline $5: 15: \odot \odot$ & 315 & 228 & 204 & 213 & 188 & 221 & 199 & 227 & 195 & 210 \\
\hline $5: 16: \odot \odot$ & 316 & 227 & 204 & 213 & 188 & 220 & 199 & 226 & 195 & 209 \\
\hline $5: 17: \odot \odot$ & 317 & 227 & 203 & 213 & 187 & 220 & 198 & 226 & 195 & 209 \\
\hline $5: 18: \odot \odot$ & 318 & 227 & 203 & 213 & 187 & 219 & 198 & 226 & 195 & 209 \\
\hline $5: 19: \odot \odot$ & 319 & 226 & 203 & 212 & 186 & 219 & 197 & 225 & 195 & 208 \\
\hline $5: 2 \odot: \odot \odot$ & 320 & 226 & 203 & 212 & 186 & 218 & 197 & 225 & 194 & 208 \\
\hline $5: 21: \odot \odot$ & 321 & 225 & 202 & 212 & 186 & 218 & 197 & 225 & 194 & 207 \\
\hline
\end{tabular}




\begin{tabular}{|c|c|c|c|c|c|c|c|c|c|c|}
\hline $5: 22: \odot \odot$ & 322 & 225 & 202 & 211 & 185 & 218 & 197 & 225 & 193 & 207 \\
\hline $5: 23: \odot \odot$ & 323 & 225 & 202 & 211 & 185 & 217 & 196 & 224 & 193 & 207 \\
\hline $5: 24: \odot \odot$ & 324 & 224 & 202 & 211 & 185 & 217 & 196 & 224 & 193 & 207 \\
\hline $5: 25: \odot \odot$ & 325 & 224 & 201 & 210 & 185 & 216 & 196 & 224 & 192 & 206 \\
\hline $5: 26: \odot \odot$ & 326 & 224 & 201 & 210 & 184 & 216 & 195 & 223 & 192 & 206 \\
\hline $5: 27: \odot \odot$ & 327 & 222 & 201 & 209 & 184 & 216 & 195 & 223 & 191 & 205 \\
\hline $5: 28: \odot \odot$ & 328 & 222 & 201 & 209 & 184 & 216 & 195 & 223 & 191 & 205 \\
\hline $5: 29: \odot \odot$ & 329 & 222 & 200 & 209 & 184 & 216 & 194 & 222 & 191 & 205 \\
\hline $5: 3 \odot: \odot \odot$ & 330 & 221 & 200 & 209 & 183 & 215 & 194 & 222 & 191 & 204 \\
\hline $5: 31: \odot \odot$ & 331 & 221 & 200 & 209 & 183 & 215 & 194 & 222 & 190 & 204 \\
\hline $5: 32: \odot \odot$ & 332 & 221 & 199 & 208 & 183 & 215 & 193 & 221 & 190 & 204 \\
\hline $5: 33: \odot \odot$ & 333 & 220 & 199 & 208 & 183 & 214 & 193 & 221 & 190 & 203 \\
\hline $5: 34: \odot \odot$ & 334 & 220 & 199 & 208 & 182 & 214 & 193 & 221 & 189 & 203 \\
\hline $5: 35: \odot \odot$ & 335 & 219 & 199 & 207 & 182 & 213 & 191 & 220 & 189 & 202 \\
\hline $5: 36: \odot \odot$ & 336 & 219 & 198 & 207 & 182 & 213 & 191 & 220 & 188 & 202 \\
\hline $5: 37: \odot \odot$ & 337 & 218 & 198 & 206 & 181 & 213 & 190 & 219 & 188 & 201 \\
\hline $5: 38: \odot \odot$ & 338 & 218 & 197 & 206 & 181 & 211 & 190 & 218 & 187 & 201 \\
\hline $5: 39: \odot \odot$ & 339 & 217 & 196 & 205 & 179 & 210 & 189 & 218 & 186 & $20 \odot$ \\
\hline $5: 4 \odot: \odot \odot$ & 340 & 217 & 196 & 204 & 179 & 210 & 189 & 217 & 185 & 200 \\
\hline $5: 41: \odot \odot$ & 341 & 216 & 195 & 204 & 178 & 209 & 188 & 215 & 185 & 199 \\
\hline $5: 42: \odot \odot$ & 342 & 216 & 193 & 203 & 178 & 209 & 188 & 215 & 184 & 198 \\
\hline $5: 43: \odot \odot$ & 343 & 216 & 193 & 203 & 177 & 209 & 187 & 214 & 182 & 198 \\
\hline $5: 44: \odot \odot$ & 344 & 215 & 192 & 202 & 177 & 208 & 187 & 214 & 182 & 197 \\
\hline $5: 45: \odot \odot$ & 345 & 215 & 192 & 202 & 176 & 208 & 186 & 213 & 181 & 197 \\
\hline $5: 46: \odot \odot$ & 346 & 214 & 191 & 200 & 175 & 207 & 186 & 212 & 181 & 196 \\
\hline $5: 47: \odot \odot$ & 347 & 214 & 191 & 199 & 175 & 207 & 185 & 212 & 180 & 195 \\
\hline $5: 48: \odot \odot$ & 348 & 213 & 190 & 199 & 174 & 206 & 185 & 211 & 180 & 195 \\
\hline $5: 49: \odot \odot$ & 349 & 213 & 190 & 198 & 173 & 206 & 184 & 211 & 180 & 194 \\
\hline $5: 5 \odot: \odot \odot$ & 350 & 211 & 189 & 198 & 172 & 205 & 184 & 210 & 179 & 194 \\
\hline $5: 51: \odot \odot$ & 351 & 211 & 189 & 197 & 172 & 205 & 183 & 210 & 179 & 193 \\
\hline $5: 52: \odot \odot$ & 352 & 211 & 189 & 197 & 171 & 205 & 183 & 209 & 178 & 193 \\
\hline $5: 53: \odot \odot$ & 353 & 210 & 188 & 196 & 171 & 204 & 182 & 209 & 178 & 192 \\
\hline $5: 54: \odot \odot$ & 354 & 210 & 188 & 196 & 171 & 204 & 182 & 209 & 178 & 192 \\
\hline $5: 55: \odot \odot$ & 355 & 209 & 187 & 195 & 170 & 203 & 181 & 208 & 177 & 191 \\
\hline $5: 56: \odot \odot$ & 356 & 209 & 187 & 195 & 170 & 203 & 181 & 208 & 177 & 191 \\
\hline $5: 57: \odot \odot$ & 357 & 209 & 187 & 194 & 169 & 202 & 181 & 207 & 177 & 191 \\
\hline $5: 58: \odot \odot$ & 358 & 209 & 186 & 194 & 169 & 202 & 180 & 207 & 176 & 190 \\
\hline $5: 59: \odot \odot$ & 359 & 208 & 186 & 194 & 169 & 200 & 180 & 207 & 176 & 190 \\
\hline $6: \odot \odot: \odot \odot$ & 360 & 208 & 185 & 193 & 168 & 200 & 179 & 205 & 176 & 189 \\
\hline $6: \odot 1: \odot \odot$ & 361 & 207 & 185 & 193 & 168 & 200 & 179 & 205 & 176 & 189 \\
\hline $6: \odot 2: \odot \odot$ & 362 & 207 & 185 & 191 & 166 & 199 & 177 & 204 & 175 & 188 \\
\hline 6:๑3:๑९ & 363 & 206 & 184 & 191 & 166 & 199 & 177 & 204 & 175 & 188 \\
\hline $6: \odot 4: \odot \odot$ & 364 & 206 & 184 & 191 & 166 & 198 & 177 & 203 & 173 & 187 \\
\hline $6: \odot 5: \odot \odot$ & 365 & 206 & 183 & $19 \odot$ & 165 & 198 & 176 & 203 & 173 & 187 \\
\hline $6: \odot 6: \odot \odot$ & 366 & 205 & 183 & 190 & 165 & 198 & 176 & 203 & 173 & 187 \\
\hline $6: \odot 7: \odot \odot$ & 367 & 205 & 183 & 190 & 165 & 198 & 176 & 202 & 172 & 187 \\
\hline 6:๑৪:๑९ & 368 & 204 & 182 & 190 & 165 & 198 & 176 & 202 & 172 & 186 \\
\hline $6: \odot 9: \odot \odot$ & 369 & 204 & 182 & 189 & 164 & 197 & 175 & 202 & 172 & 186 \\
\hline $6: 10: \odot \odot$ & 370 & 204 & 182 & 189 & 164 & 197 & 175 & 201 & 171 & 185 \\
\hline $6: 11: \odot \odot$ & 371 & 203 & 182 & 189 & 164 & 197 & 175 & 201 & 171 & 185 \\
\hline $6: 12: \odot \odot$ & 372 & 203 & 181 & 188 & 163 & 196 & 174 & 201 & 171 & 185 \\
\hline $6: 13: \odot \odot$ & 373 & 203 & 181 & 188 & 163 & 196 & 174 & 201 & 170 & 184 \\
\hline $6: 14: \odot \odot$ & 374 & 202 & 179 & 188 & 163 & 196 & 174 & 200 & 170 & 184 \\
\hline $6: 15: \odot \odot$ & 375 & 202 & 179 & 188 & 163 & 196 & 173 & 200 & 170 & 184 \\
\hline $6: 16: \odot \odot$ & 376 & 202 & 179 & 187 & 162 & 195 & 173 & 199 & 170 & 183 \\
\hline $6: 17: \odot \odot$ & 377 & 200 & 178 & 187 & 162 & 195 & 173 & 199 & 169 & 183 \\
\hline $6: 18: \odot \odot$ & 378 & 200 & 178 & 186 & 162 & 195 & 172 & 198 & 169 & 183 \\
\hline
\end{tabular}




\begin{tabular}{|c|c|c|c|c|c|c|c|c|c|c|}
\hline $6: 19: \odot \odot$ & 379 & 199 & 178 & 186 & 162 & 195 & 172 & 198 & 169 & 182 \\
\hline $6: 20: \odot \odot$ & 380 & 199 & 177 & 186 & 161 & 194 & 171 & 197 & 168 & 182 \\
\hline $6: 21: \odot \odot$ & 381 & 198 & 177 & 185 & 161 & 194 & 171 & 197 & 168 & 181 \\
\hline $6: 22: \odot \odot$ & 382 & 198 & 177 & 185 & 161 & 193 & 171 & 195 & 168 & 181 \\
\hline $6: 23: \odot \odot$ & 383 & 197 & 176 & 185 & 160 & 193 & 170 & 195 & 167 & 180 \\
\hline $6: 24: \odot \odot$ & 384 & 197 & 176 & 185 & 160 & 193 & 170 & 194 & 167 & 180 \\
\hline $6: 25: \odot \odot$ & 385 & 197 & 175 & 184 & 159 & 192 & 170 & 194 & 167 & 180 \\
\hline $6: 26: \odot \odot$ & 386 & 196 & 175 & 184 & 159 & 192 & 169 & 193 & 166 & 179 \\
\hline $6: 27: \odot \odot$ & 387 & 196 & 175 & 184 & 159 & 192 & 169 & 193 & 166 & 179 \\
\hline $6: 28: \odot \odot$ & 388 & 195 & 175 & 182 & 159 & 191 & 169 & 193 & 165 & 179 \\
\hline $6: 29: \odot \odot$ & 389 & 195 & 174 & 182 & 158 & 191 & 168 & 193 & 165 & 178 \\
\hline $6: 3 \odot: \odot \odot$ & 390 & 195 & 174 & 182 & 158 & 191 & 168 & 193 & 165 & 178 \\
\hline $6: 31: \odot \odot$ & 391 & 195 & 174 & 181 & 158 & 191 & 168 & 192 & 165 & 178 \\
\hline $6: 32: \odot \odot$ & 392 & 194 & 174 & 181 & 158 & 190 & 168 & 192 & 164 & 178 \\
\hline $6: 33: \odot \odot$ & 393 & 194 & 173 & 181 & 158 & 190 & 168 & 192 & 164 & 177 \\
\hline $6: 34: \odot \odot$ & 394 & 194 & 173 & 181 & 158 & 190 & 168 & 191 & 164 & 177 \\
\hline $6: 35: \odot \odot$ & 395 & 194 & 173 & 180 & 157 & 190 & 166 & 191 & 164 & 177 \\
\hline $6: 36: \odot \odot$ & 396 & 193 & 173 & 180 & 157 & 189 & 166 & 191 & 164 & 177 \\
\hline $6: 37: \odot \odot$ & 397 & 193 & 173 & 180 & 157 & 189 & 166 & 191 & 163 & 176 \\
\hline $6: 38: \odot \odot$ & 398 & 193 & 172 & 180 & 157 & 189 & 165 & 190 & 163 & 176 \\
\hline $6: 39: \odot \odot$ & 399 & 192 & 172 & 179 & 155 & 188 & 165 & 190 & 163 & 175 \\
\hline $6: 4 \odot: \odot \odot$ & 400 & 192 & 172 & 179 & 155 & 188 & 164 & 190 & 163 & 175 \\
\hline $6: 41: \odot \odot$ & 401 & 192 & 172 & 179 & 155 & 188 & 164 & 190 & 162 & 175 \\
\hline $6: 42: \odot \odot$ & $4 \odot 2$ & 191 & 171 & 179 & 155 & 186 & 164 & 189 & 162 & 175 \\
\hline $6: 43: \odot \odot$ & 403 & 191 & 171 & 178 & 154 & 186 & 164 & 189 & 161 & 174 \\
\hline $6: 44: \odot \odot$ & 404 & 191 & 170 & 178 & 154 & 186 & 163 & 188 & 161 & 174 \\
\hline $6: 45: \odot \odot$ & 405 & 190 & 170 & 178 & 154 & 186 & 163 & 188 & 161 & 174 \\
\hline $6: 46: \odot \odot$ & 406 & $19 \odot$ & 170 & 177 & 154 & 185 & 163 & 188 & 161 & 173 \\
\hline $6: 47: \odot \odot$ & $4 \odot 7$ & 190 & 170 & 177 & 154 & 185 & 163 & 186 & 159 & 173 \\
\hline $6: 48: \odot \odot$ & 408 & $19 \odot$ & 170 & 177 & 154 & 185 & 163 & 186 & 159 & 173 \\
\hline $6: 49: \odot \odot$ & 409 & 188 & 168 & 177 & 153 & 185 & 163 & 186 & 159 & 173 \\
\hline $6: 5 \odot: \odot \odot$ & 410 & 188 & 168 & 176 & 153 & 185 & 162 & 186 & 158 & 172 \\
\hline $6: 51: \odot \odot$ & 411 & 187 & 167 & 176 & 152 & 184 & 162 & 185 & 158 & 172 \\
\hline $6: 52: \odot \odot$ & 412 & 187 & 167 & 175 & 152 & 184 & 162 & 185 & 158 & 171 \\
\hline $6: 53: \odot \odot$ & 413 & 187 & 167 & 175 & 152 & 184 & 162 & 185 & 158 & 171 \\
\hline $6: 54: \odot \odot$ & 414 & 187 & 167 & 175 & 152 & 184 & 162 & 185 & 158 & 171 \\
\hline $6: 55: \odot \odot$ & 415 & 186 & 167 & 175 & 152 & 184 & 162 & 184 & 157 & 171 \\
\hline $6: 56: \odot \odot$ & 416 & 186 & 167 & 175 & 152 & 183 & 161 & 184 & 157 & 171 \\
\hline $6: 57: \odot \odot$ & 417 & 186 & 166 & 175 & 152 & 183 & 161 & 184 & 157 & 171 \\
\hline $6: 58: \odot \odot$ & 418 & 186 & 166 & 174 & 151 & 183 & 161 & 183 & 157 & 170 \\
\hline $6: 59: \odot \odot$ & 419 & 185 & 166 & 174 & 151 & 182 & 160 & 183 & 157 & 170 \\
\hline 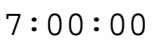 & 420 & 185 & 166 & 174 & 151 & 182 & 160 & 183 & 156 & 170 \\
\hline $7: \odot 1: \odot \odot$ & 421 & 185 & 165 & 173 & 150 & 182 & 160 & 183 & 156 & 169 \\
\hline $7: \odot 2: \odot \odot$ & 422 & 184 & 165 & 173 & 150 & 182 & 159 & 182 & 155 & 169 \\
\hline $7: \odot 3: \odot \odot$ & 423 & 184 & 165 & 173 & 150 & 181 & 159 & 182 & 155 & 169 \\
\hline $7: \odot 4: \odot \odot$ & 424 & 184 & 164 & 172 & 150 & 181 & 159 & 181 & 155 & 168 \\
\hline $7: \odot 5: \odot \odot$ & 425 & 183 & 164 & 172 & 149 & 181 & 159 & 181 & 154 & 168 \\
\hline $7: \odot 6: \odot \odot$ & 426 & 183 & 164 & 172 & 149 & 181 & 157 & 181 & 154 & 168 \\
\hline $7: \odot 7: \odot \odot$ & 427 & 183 & 163 & 171 & 149 & 180 & 157 & 180 & 154 & 167 \\
\hline 7:๑৪:๑९ & 428 & 182 & 163 & 171 & 148 & 180 & 157 & 180 & 154 & 167 \\
\hline 7:०9:๑९ & 429 & 182 & 163 & 171 & 148 & 180 & 156 & 180 & 153 & 167 \\
\hline $7: 10: \odot \odot$ & 430 & 182 & 162 & 171 & 148 & 179 & 156 & 179 & 153 & 166 \\
\hline $7: 11: 0 \odot$ & 431 & 181 & 162 & 170 & 147 & 179 & 155 & 179 & 153 & 166 \\
\hline $7: 12: \odot \odot$ & 432 & 181 & 162 & 170 & 147 & 179 & 155 & 179 & 153 & 166 \\
\hline $7: 13: \odot \odot$ & 433 & 180 & 161 & 170 & 147 & 178 & 155 & 177 & 152 & 165 \\
\hline $7: 14: \odot \odot$ & 434 & 180 & 161 & 170 & 147 & 178 & 154 & 177 & 152 & 165 \\
\hline $7: 15: \odot \odot$ & 435 & 180 & 161 & 168 & 146 & 178 & 154 & 176 & 152 & 164 \\
\hline
\end{tabular}




\begin{tabular}{|c|c|c|c|c|c|c|c|c|c|c|}
\hline $7: 16: 0 \odot$ & 436 & 179 & 161 & 168 & 146 & 178 & 154 & 176 & 152 & 164 \\
\hline $7: 17: \odot \odot$ & 437 & 179 & 159 & 168 & 146 & 177 & 154 & 176 & 151 & 164 \\
\hline $7: 18: \odot \odot$ & 438 & 179 & 159 & 168 & 146 & 177 & 154 & 176 & 151 & 164 \\
\hline $7: 19: \odot \odot$ & 439 & 177 & 159 & 167 & 146 & 177 & 154 & 175 & 151 & 163 \\
\hline $7: 2 \odot: \odot \odot$ & 440 & 177 & 159 & 167 & 146 & 176 & 153 & 175 & 151 & 163 \\
\hline $7: 21: \odot \odot$ & 441 & 177 & 158 & 167 & 145 & 176 & 153 & 174 & 151 & 163 \\
\hline $7: 22: \odot \odot$ & 442 & 177 & 158 & 167 & 145 & 176 & 153 & 174 & 150 & 163 \\
\hline $7: 23: \odot \odot$ & 443 & 176 & 158 & 166 & 145 & 175 & 153 & 174 & 150 & 162 \\
\hline $7: 24: \odot \odot$ & 444 & 176 & 158 & 166 & 145 & 175 & 152 & 174 & 150 & 162 \\
\hline $7: 25: \odot \odot$ & 445 & 176 & 158 & 166 & 145 & 175 & 152 & 174 & 150 & 162 \\
\hline $7: 26: \odot \odot$ & 446 & 176 & 157 & 166 & 145 & 175 & 152 & 174 & 150 & 162 \\
\hline $7: 27: \odot \odot$ & 447 & 176 & 157 & 166 & 145 & 173 & 152 & 174 & 150 & 162 \\
\hline $7: 28: \odot \odot$ & 448 & 175 & 157 & 165 & 144 & 173 & 152 & 173 & 149 & 161 \\
\hline $7: 29: \odot \odot$ & 449 & 175 & 157 & 165 & 144 & 173 & 152 & 173 & 149 & 161 \\
\hline $7: 3 \odot: \odot \odot$ & 450 & 175 & 157 & 165 & 144 & 173 & 152 & 173 & 149 & 161 \\
\hline $7: 31: \odot \odot$ & 451 & 175 & 157 & 165 & 144 & 173 & 151 & 173 & 149 & 161 \\
\hline $7: 32: \odot \odot$ & 452 & 174 & 156 & 165 & 144 & 173 & 151 & 173 & 149 & 161 \\
\hline $7: 33: \odot \odot$ & 453 & 174 & 156 & 165 & 143 & 172 & 151 & 172 & 149 & 160 \\
\hline $7: 34: \odot \odot$ & 454 & 174 & 156 & 164 & 143 & 172 & 151 & 172 & 148 & 160 \\
\hline $7: 35: \odot \odot$ & 455 & 174 & 156 & 164 & 143 & 172 & 151 & 172 & 148 & 160 \\
\hline $7: 36: \odot \odot$ & 456 & 173 & 156 & 164 & 143 & 172 & 150 & 171 & 148 & 160 \\
\hline $7: 37: \odot \odot$ & 457 & 173 & 155 & 163 & 143 & 171 & 150 & 171 & 146 & 159 \\
\hline $7: 38: \odot \odot$ & 458 & 173 & 155 & 163 & 141 & 171 & 150 & 171 & 146 & 159 \\
\hline $7: 39: \odot \odot$ & 459 & 173 & 155 & 163 & 141 & 171 & 150 & 170 & 146 & 159 \\
\hline $7: 4 \odot: \odot \odot$ & 460 & 172 & 155 & 163 & 141 & 171 & 150 & 170 & 146 & 159 \\
\hline $7: 41: \odot \odot$ & 461 & 172 & 155 & 162 & 141 & 171 & 150 & 170 & 145 & 158 \\
\hline $7: 42: \odot \odot$ & 462 & 172 & 154 & 162 & 141 & 170 & 149 & 169 & 145 & 158 \\
\hline $7: 43: \odot \odot$ & 463 & 172 & 154 & 162 & 141 & 170 & 149 & 169 & 145 & 158 \\
\hline $7: 44: \odot \odot$ & 464 & 171 & 154 & 162 & 141 & 170 & 149 & 169 & 145 & 158 \\
\hline $7: 45: \odot \odot$ & 465 & 171 & 154 & 162 & 140 & 170 & 149 & 169 & 144 & 157 \\
\hline $7: 46: \odot \odot$ & 466 & 171 & 153 & 161 & 140 & 169 & 148 & 168 & 144 & 157 \\
\hline $7: 47: \odot \odot$ & 467 & 171 & 153 & 161 & 140 & 169 & 148 & 168 & 144 & 157 \\
\hline $7: 48: \odot \odot$ & 468 & 170 & 153 & 161 & 140 & 169 & 148 & 168 & 144 & 157 \\
\hline $7: 49: \odot \odot$ & 469 & 170 & 152 & 161 & 139 & 169 & 148 & 168 & 143 & 156 \\
\hline $7: 5 \odot: \odot \odot$ & 470 & 170 & 152 & 160 & 139 & 168 & 146 & 166 & 143 & 156 \\
\hline $7: 51: \odot \odot$ & 471 & 169 & 152 & 160 & 139 & 168 & 146 & 166 & 143 & 155 \\
\hline $7: 52: \odot \odot$ & 472 & 169 & 152 & 160 & 139 & 168 & 146 & 166 & 143 & 155 \\
\hline $7: 53: \odot \odot$ & 473 & 169 & 151 & 160 & 139 & 167 & 146 & 166 & 143 & 155 \\
\hline $7: 54: \odot \odot$ & 474 & 168 & 151 & 159 & 138 & 167 & 145 & 165 & 142 & 154 \\
\hline $7: 55: \odot \odot$ & 475 & 168 & 151 & 159 & 138 & 167 & 145 & 165 & 142 & 154 \\
\hline $7: 56: \odot \odot$ & 476 & 168 & 150 & 159 & 138 & 167 & 145 & 165 & 141 & 154 \\
\hline $7: 57: \odot \odot$ & 477 & 168 & 150 & 159 & 138 & 166 & 145 & 165 & 141 & 154 \\
\hline $7: 58: \odot \odot$ & 478 & 166 & 150 & 158 & 138 & 166 & 144 & 164 & 141 & 153 \\
\hline $7: 59: \odot \odot$ & 479 & 166 & 150 & 158 & 137 & 166 & 144 & 164 & 141 & 153 \\
\hline ৪: ๑९:๑๑ & 480 & 166 & 150 & 158 & 137 & 166 & 144 & 164 & 141 & 153 \\
\hline $8: 01: 0 \odot$ & 481 & 166 & 148 & 158 & 137 & 166 & 144 & 164 & 141 & 153 \\
\hline ৪: $\odot 2: \odot \odot$ & 482 & 165 & 148 & 158 & 137 & 166 & 144 & 164 & 141 & 153 \\
\hline ৪: ๑3:๑९ & 483 & 165 & 148 & 157 & 137 & 166 & 144 & 164 & 140 & 153 \\
\hline ৪: ๑4:๑९ & 484 & 165 & 148 & 157 & 136 & 165 & 143 & 163 & 140 & 152 \\
\hline 8: $\odot 5: \odot \odot$ & 485 & 165 & 148 & 157 & 136 & 165 & 143 & 163 & 140 & 152 \\
\hline 8:०6:๑९ & 486 & 165 & 148 & 157 & 136 & 165 & 143 & 163 & 140 & 152 \\
\hline ৪:०7:๑९ & 487 & 164 & 148 & 157 & 136 & 165 & 143 & 163 & 140 & 152 \\
\hline ৪: ๑৪:๑९ & 488 & 164 & 147 & 155 & 136 & 164 & 143 & 163 & 140 & 152 \\
\hline ৪: ๑९:๑९ & 489 & 164 & 147 & 155 & 136 & 164 & 143 & 163 & 140 & 152 \\
\hline $8: 10: \odot \odot$ & 490 & 163 & 147 & 154 & 135 & 164 & 142 & 162 & 139 & 151 \\
\hline $8: 11: \odot \odot$ & 491 & 163 & 147 & 154 & 135 & 164 & 142 & 162 & 139 & 151 \\
\hline $8: 12: \odot \odot$ & 492 & 163 & 146 & 154 & 135 & 164 & 142 & 162 & 139 & 151 \\
\hline
\end{tabular}




\begin{tabular}{|c|c|c|c|c|c|c|c|c|c|c|}
\hline ৪:13:๑९ & 493 & 163 & 146 & 154 & 135 & 163 & 142 & 161 & 139 & 150 \\
\hline $8: 14: \odot \odot$ & 494 & 163 & 146 & 153 & 135 & 163 & 142 & 161 & 138 & 150 \\
\hline $8: 15: \odot \odot$ & 495 & 162 & 146 & 153 & 135 & 163 & 141 & 161 & 138 & 150 \\
\hline $8: 16: \odot \odot$ & 496 & 162 & 145 & 153 & 134 & 162 & 141 & 161 & 138 & 150 \\
\hline $8: 17: \odot \odot$ & 497 & 162 & 145 & 153 & 134 & 162 & 141 & 160 & 138 & 149 \\
\hline 8:18:๑๑ & 498 & 162 & 145 & 153 & 134 & 162 & 141 & 160 & 138 & 149 \\
\hline $8: 19: \odot \odot$ & 499 & 162 & 145 & 152 & 134 & 162 & 141 & 160 & 138 & 149 \\
\hline ৪: $2 \odot: \odot \odot$ & 500 & 162 & 145 & 152 & 134 & 162 & 141 & 160 & 138 & 149 \\
\hline $8: 21: \odot \odot$ & 501 & 161 & 145 & 152 & 134 & 161 & 140 & 160 & 137 & 149 \\
\hline $8: 22: \odot \odot$ & 502 & 161 & 145 & 152 & 134 & 161 & 140 & 160 & 137 & 149 \\
\hline $8: 23: \odot \odot$ & 503 & 161 & 144 & 152 & 133 & 161 & 140 & 160 & 137 & 148 \\
\hline $8: 24: \odot \odot$ & 504 & 161 & 144 & 152 & 133 & 161 & 140 & 159 & 137 & 148 \\
\hline $8: 25: \odot \odot$ & 505 & 161 & 144 & 152 & 133 & 161 & 140 & 159 & 137 & 148 \\
\hline $8: 26: \odot \odot$ & 506 & 160 & 144 & 152 & 133 & 159 & 140 & 159 & 137 & 148 \\
\hline $8: 27: \odot \odot$ & 507 & 160 & 144 & 151 & 133 & 159 & 140 & 159 & 136 & 148 \\
\hline $8: 28: \odot \odot$ & 508 & 160 & 144 & 151 & 133 & 159 & 140 & 159 & 136 & 148 \\
\hline ৪:29:๑९ & 509 & 160 & 143 & 151 & 133 & 159 & 139 & 158 & 136 & 147 \\
\hline ৪: $3 \odot: \odot \odot$ & 510 & $16 \odot$ & 143 & 151 & 133 & 159 & 139 & 158 & 136 & 147 \\
\hline 8: $31: \odot \odot$ & 511 & 160 & 143 & 151 & 133 & 159 & 139 & 158 & 136 & 147 \\
\hline $8: 32: \odot \odot$ & 512 & 159 & 143 & 150 & 133 & 158 & 139 & 158 & 136 & 147 \\
\hline 8: 33:๑९ & 513 & 159 & 143 & 150 & 132 & 158 & 139 & 158 & 136 & 147 \\
\hline $8: 34: \odot \odot$ & 514 & 159 & 143 & 150 & 132 & 158 & 138 & 158 & 136 & 147 \\
\hline $8: 35: \odot \odot$ & 515 & 159 & 143 & 150 & 132 & 158 & 138 & 158 & 136 & 147 \\
\hline ৪: $36: \odot \odot$ & 516 & 159 & 142 & 150 & 132 & 158 & 138 & 157 & 135 & 146 \\
\hline $8: 37: \odot \odot$ & 517 & 159 & 142 & 150 & 132 & 158 & 138 & 157 & 135 & 146 \\
\hline $8: 38: \odot \odot$ & 518 & 158 & 142 & 150 & 132 & 157 & 138 & 157 & 135 & 146 \\
\hline ৪: $39: \odot \odot$ & 519 & 158 & 142 & 149 & 132 & 157 & 138 & 157 & 135 & 146 \\
\hline 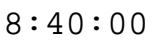 & 520 & 158 & 142 & 149 & 131 & 157 & 138 & 157 & 135 & 146 \\
\hline $8: 41: \odot \odot$ & 521 & 158 & 142 & 149 & 131 & 157 & 138 & 157 & 135 & 146 \\
\hline $8: 42: \odot \odot$ & 522 & 157 & 142 & 149 & 131 & 157 & 137 & 156 & 134 & 145 \\
\hline $8: 43: \odot \odot$ & 523 & 157 & 142 & 149 & 131 & 156 & 137 & 156 & 134 & 145 \\
\hline $8: 44: \odot \odot$ & 524 & 157 & 141 & 149 & 131 & 156 & 137 & 156 & 134 & 145 \\
\hline $8: 45: \odot \odot$ & 525 & 157 & 141 & 148 & 131 & 156 & 137 & 156 & 134 & 145 \\
\hline $8: 46: \odot \odot$ & 526 & 157 & 141 & 148 & 130 & 156 & 137 & 155 & 134 & 145 \\
\hline $8: 47: \odot \odot$ & 527 & 156 & 141 & 148 & 130 & 156 & 136 & 155 & 134 & 144 \\
\hline $8: 48: \odot \odot$ & 528 & 156 & 141 & 148 & 130 & 156 & 136 & 155 & 132 & 144 \\
\hline $8: 49: \odot \odot$ & 529 & 156 & 141 & 148 & 130 & 155 & 136 & 155 & 132 & 144 \\
\hline $8: 5 \odot: \odot \odot$ & 530 & 156 & 140 & 148 & 130 & 155 & 136 & 155 & 132 & 144 \\
\hline $8: 51: \odot \odot$ & 531 & 156 & 140 & 148 & 130 & 155 & 136 & 154 & 132 & 144 \\
\hline $8: 52: \odot \odot$ & 532 & 156 & 140 & 148 & 130 & 155 & 136 & 154 & 132 & 144 \\
\hline $8: 53: \odot \odot$ & 533 & 155 & 140 & 148 & 130 & 155 & 136 & 154 & 132 & 144 \\
\hline $8: 54: \odot \odot$ & 534 & 155 & 140 & 147 & 130 & 155 & 136 & 154 & 132 & 143 \\
\hline $8: 55: \odot \odot$ & 535 & 155 & 140 & 147 & 130 & 155 & 136 & 154 & 131 & 143 \\
\hline $8: 56: \odot \odot$ & 536 & 155 & 140 & 147 & 130 & 154 & 135 & 154 & 131 & 143 \\
\hline $8: 57: \odot \odot$ & 537 & 154 & 139 & 147 & 128 & 154 & 135 & 152 & 131 & 142 \\
\hline $8: 58: \odot \odot$ & 538 & 154 & 139 & 147 & 128 & 154 & 135 & 152 & 131 & 142 \\
\hline $8: 59: \odot \odot$ & 539 & 154 & 139 & 147 & 128 & 154 & 135 & 152 & 131 & 142 \\
\hline 9:०९:๑९ & 540 & 154 & 139 & 147 & 128 & 153 & 135 & 152 & 131 & 142 \\
\hline $9: \odot 1: \odot \odot$ & 541 & 154 & 139 & 146 & 128 & 153 & 134 & 152 & 131 & 142 \\
\hline $9: \odot 2: \odot \odot$ & 542 & 154 & 139 & 146 & 128 & 153 & 134 & 152 & 131 & 142 \\
\hline 9:०3:๑९ & 543 & 152 & 138 & 146 & 127 & 153 & 134 & 151 & 130 & 141 \\
\hline $9: \odot 4: \odot \odot$ & 544 & 152 & 138 & 146 & 127 & 152 & 134 & 151 & 130 & 141 \\
\hline $9: \odot 5: \odot \odot$ & 545 & 152 & 138 & 145 & 127 & 152 & 134 & 151 & 130 & 141 \\
\hline 9:๑6:๑९ & 546 & 152 & 138 & 145 & 127 & 152 & 134 & 151 & 130 & 141 \\
\hline $9: \odot 7: \odot \odot$ & 547 & 152 & 138 & 145 & 127 & 152 & 134 & 151 & 130 & 141 \\
\hline 9:๑৪:๑९ & 548 & 151 & 137 & 145 & 127 & 152 & 132 & 150 & 130 & 141 \\
\hline 9:๑९:๑९ & 549 & 151 & 137 & 145 & 127 & 152 & 132 & 150 & 130 & 141 \\
\hline
\end{tabular}




\begin{tabular}{|c|c|c|c|c|c|c|c|c|c|c|}
\hline $9: 10: \odot \odot$ & 550 & 151 & 137 & 143 & 126 & 151 & 132 & 150 & 130 & 140 \\
\hline $9: 11: \odot \odot$ & 551 & 151 & 137 & 143 & 126 & 151 & 132 & 150 & 130 & 140 \\
\hline $9: 12: \odot \odot$ & 552 & 151 & 137 & 143 & 126 & 151 & 132 & 150 & 129 & 140 \\
\hline $9: 13: \odot \odot$ & 553 & 150 & 137 & 143 & 126 & 151 & 132 & 150 & 129 & 140 \\
\hline $9: 14: \odot \odot$ & 554 & 150 & 137 & 143 & 126 & 151 & 131 & 150 & 129 & 140 \\
\hline $9: 15: \odot \odot$ & 555 & 150 & 137 & 143 & 126 & 151 & 131 & 150 & 129 & 140 \\
\hline $9: 16: \odot \odot$ & 556 & $15 \odot$ & 137 & 143 & 126 & 151 & 131 & 150 & 129 & 140 \\
\hline $9: 17: \odot \odot$ & 557 & 150 & 137 & 143 & 126 & 151 & 131 & 150 & 129 & 140 \\
\hline $9: 18: \odot \odot$ & 558 & 150 & 136 & 142 & 126 & 150 & 131 & 149 & 128 & 139 \\
\hline $9: 19: \odot \odot$ & 559 & $15 \odot$ & 136 & 142 & 126 & 150 & 131 & 149 & 128 & 139 \\
\hline $9: 2 \odot: \odot \odot$ & 560 & $15 \odot$ & 136 & 142 & 126 & 150 & 131 & 149 & 128 & 139 \\
\hline $9: 21: \odot \odot$ & 561 & 149 & 136 & 142 & 126 & 150 & 131 & 149 & 128 & 139 \\
\hline $9: 22: \odot \odot$ & 562 & 149 & 136 & 142 & 125 & 149 & 131 & 149 & 128 & 139 \\
\hline $9: 23: 0 \odot$ & 563 & 149 & 136 & 142 & 125 & 149 & 130 & 148 & 128 & 138 \\
\hline $9: 24: \odot \odot$ & 564 & 149 & 134 & 141 & 125 & 149 & 130 & 148 & 127 & 138 \\
\hline $9: 25: \odot \odot$ & 565 & 149 & 134 & 141 & 125 & 149 & 130 & 148 & 127 & 138 \\
\hline $9: 26: \odot \odot$ & 566 & 149 & 134 & 141 & 125 & 149 & 130 & 148 & 127 & 138 \\
\hline $9: 27: \odot \odot$ & 567 & 148 & 134 & 141 & 125 & 149 & 130 & 148 & 127 & 138 \\
\hline $9: 28: \odot \odot$ & 568 & 148 & 134 & 141 & 125 & 149 & 130 & 148 & 127 & 138 \\
\hline $9: 29: \odot \odot$ & 569 & 148 & 134 & 141 & 125 & 149 & 130 & 147 & 127 & 138 \\
\hline $9: 3 \odot: \odot \odot$ & 570 & 148 & 134 & 140 & 125 & 148 & 130 & 147 & 127 & 137 \\
\hline $9: 31: \odot \odot$ & 571 & 148 & 134 & 140 & 124 & 148 & 130 & 147 & 127 & 137 \\
\hline $9: 32: 0 \odot$ & 572 & 148 & 133 & 140 & 124 & 148 & 130 & 147 & 127 & 137 \\
\hline $9: 33: \odot \odot$ & 573 & 147 & 133 & 140 & 124 & 148 & 130 & 147 & 127 & 137 \\
\hline $9: 34: \odot \odot$ & 574 & 147 & 133 & 140 & 124 & 148 & 129 & 147 & 126 & 137 \\
\hline $9: 35: \odot \odot$ & 575 & 147 & 133 & 140 & 124 & 148 & 129 & 147 & 126 & 137 \\
\hline $9: 36: \odot \odot$ & 576 & 147 & 133 & 140 & 124 & 148 & 129 & 147 & 126 & 137 \\
\hline $9: 37: \odot \odot$ & 577 & 147 & 133 & 139 & 124 & 148 & 129 & 146 & 126 & 137 \\
\hline $9: 38: \odot \odot$ & 578 & 147 & 133 & 139 & 124 & 146 & 129 & 146 & 126 & 136 \\
\hline $9: 39: \odot \odot$ & 579 & 147 & 133 & 139 & 124 & 146 & 129 & 146 & 126 & 136 \\
\hline $9: 4 \odot: \odot \odot$ & 580 & 146 & 132 & 139 & 123 & 146 & 128 & 146 & 126 & 136 \\
\hline $9: 41: \odot \odot$ & 581 & 146 & 132 & 139 & 123 & 146 & 128 & 145 & 125 & 136 \\
\hline $9: 42: \odot \odot$ & 582 & 146 & 132 & 139 & 123 & 146 & 128 & 145 & 125 & 136 \\
\hline $9: 43: \odot \odot$ & 583 & 146 & 132 & 139 & 123 & 146 & 128 & 145 & 125 & 136 \\
\hline $9: 44: \odot \odot$ & 584 & 146 & 132 & 138 & 123 & 145 & 128 & 145 & 125 & 135 \\
\hline $9: 45: \odot \odot$ & 585 & 146 & 132 & 138 & 123 & 145 & 128 & 145 & 125 & 135 \\
\hline $9: 46: \odot \odot$ & 586 & 145 & 131 & 138 & 123 & 145 & 127 & 144 & 125 & 135 \\
\hline $9: 47: \odot \odot$ & 587 & 145 & 131 & 138 & 122 & 145 & 127 & 144 & 125 & 135 \\
\hline $9: 48: \odot \odot$ & 588 & 145 & 131 & 138 & 122 & 145 & 127 & 144 & 125 & 135 \\
\hline $9: 49: \odot \odot$ & 589 & 145 & 131 & 138 & 122 & 144 & 127 & 144 & 125 & 135 \\
\hline 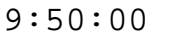 & $59 \odot$ & 145 & 131 & 137 & 122 & 144 & 127 & 144 & 124 & 134 \\
\hline $9: 51: \odot \odot$ & 591 & 145 & 131 & 137 & 122 & 144 & 127 & 144 & 124 & 134 \\
\hline $9: 52: \odot \odot$ & 592 & 144 & 131 & 137 & 122 & 144 & 127 & 144 & 124 & 134 \\
\hline 9: $53: \odot \odot$ & 593 & 144 & 130 & 137 & 122 & 144 & 127 & 144 & 124 & 134 \\
\hline $9: 54: \odot \odot$ & 594 & 144 & 130 & 137 & 121 & 143 & 126 & 143 & 124 & 133 \\
\hline $9: 55: \odot \odot$ & 595 & 144 & 130 & 137 & 121 & 143 & 126 & 143 & 124 & 133 \\
\hline $9: 56: \odot \odot$ & 596 & 144 & 130 & 137 & 121 & 143 & 126 & 143 & 124 & 133 \\
\hline $9: 57: \odot \odot$ & 597 & 144 & 130 & 137 & 121 & 143 & 126 & 143 & 123 & 133 \\
\hline $9: 58: \odot \odot$ & 598 & 143 & 130 & 137 & 121 & 143 & 126 & 143 & 123 & 133 \\
\hline 9:59:๑९ & 599 & 143 & 130 & 136 & 121 & 143 & 126 & 143 & 123 & 133 \\
\hline 1०:०๑:०๑ & 600 & 143 & 130 & 136 & 121 & 142 & 125 & 143 & 123 & 133 \\
\hline $10: 01: \odot \odot$ & 601 & 143 & 129 & 136 & 121 & 142 & 125 & 143 & 123 & 133 \\
\hline $1 \odot: \odot 2: \odot \odot$ & 602 & 143 & 129 & 136 & 120 & 142 & 125 & 142 & 123 & 132 \\
\hline 1०: $: 3: \odot \odot$ & 603 & 143 & 129 & 136 & 120 & 142 & 125 & 142 & 123 & 132 \\
\hline 1०: $\odot 4: \odot \odot$ & 604 & 141 & 129 & 135 & 120 & 142 & 125 & 142 & 123 & 132 \\
\hline $10: \odot 5: \odot \odot$ & 605 & 141 & 129 & 135 & 120 & 142 & 125 & 142 & 123 & 132 \\
\hline 1०: ๑6:๑९ & 606 & 141 & 129 & 135 & 120 & 141 & 125 & 142 & 123 & 132 \\
\hline
\end{tabular}




\begin{tabular}{|c|c|c|c|c|c|c|c|c|c|c|}
\hline $10: 07: \odot \odot$ & 607 & 141 & 129 & 135 & 120 & 141 & 125 & 142 & 123 & 132 \\
\hline 1०: $९ 8: \odot \odot$ & 608 & 141 & 129 & 135 & 120 & 141 & 124 & 142 & 122 & 132 \\
\hline 1०: $\odot 9: \odot \odot$ & 609 & 141 & 128 & 135 & 120 & 141 & 124 & 141 & 122 & 131 \\
\hline $10: 10: \odot \odot$ & 610 & 141 & 128 & 135 & 119 & 141 & 124 & 141 & 122 & 131 \\
\hline $10: 11: \odot \odot$ & 611 & 141 & 128 & 135 & 119 & 141 & 124 & 141 & 122 & 131 \\
\hline $10: 12: \odot \odot$ & 612 & 140 & 128 & 134 & 119 & 141 & 124 & 141 & 122 & 131 \\
\hline $10: 13: \odot \odot$ & 613 & 140 & 128 & 134 & 119 & 141 & 124 & 141 & 122 & 131 \\
\hline $10: 14: \odot \odot$ & 614 & 140 & 128 & 134 & 119 & 140 & 123 & 141 & 122 & 131 \\
\hline $10: 15: \odot \odot$ & 615 & 140 & 128 & 134 & 119 & 140 & 123 & 141 & 122 & 131 \\
\hline $10: 16: \odot \odot$ & 616 & 140 & 128 & 134 & 119 & 140 & 123 & 139 & 122 & 131 \\
\hline $10: 17: \odot \odot$ & 617 & 140 & 128 & 134 & 119 & 140 & 123 & 139 & 122 & 131 \\
\hline $10: 18: \odot \odot$ & 618 & 140 & 128 & 134 & 119 & 140 & 123 & 139 & 122 & 131 \\
\hline $10: 19: \odot \odot$ & 619 & 140 & 128 & 134 & 119 & 140 & 123 & 139 & 121 & 130 \\
\hline $10: 20: \odot \odot$ & 620 & 139 & 127 & 134 & 119 & 140 & 123 & 139 & 121 & 130 \\
\hline $10: 21: 0 \odot$ & 621 & 139 & 127 & 134 & 119 & 140 & 123 & 139 & 121 & 130 \\
\hline $10: 22: \odot \odot$ & 622 & 139 & 127 & 134 & 119 & 140 & 123 & 139 & 121 & 130 \\
\hline $10: 23: \odot \odot$ & 623 & 139 & 127 & 134 & 119 & 139 & 123 & 138 & 121 & 130 \\
\hline $10: 24: \odot \odot$ & 624 & 139 & 127 & 133 & 118 & 139 & 122 & 138 & 121 & 129 \\
\hline $10: 25: \odot \odot$ & 625 & 139 & 127 & 133 & 118 & 139 & 122 & 138 & 121 & 129 \\
\hline $10: 26: \odot \odot$ & 626 & 139 & 127 & 133 & 118 & 139 & 122 & 138 & 121 & 129 \\
\hline $10: 27: \odot \odot$ & 627 & 139 & 127 & 133 & 118 & 139 & 122 & 138 & 121 & 129 \\
\hline $10: 28: 0 \odot$ & 628 & 139 & 127 & 133 & 118 & 139 & 122 & 138 & 121 & 129 \\
\hline $10: 29: \odot \odot$ & 629 & 139 & 127 & 133 & 118 & 139 & 122 & 138 & 121 & 129 \\
\hline $10: 30: \odot \odot$ & 630 & 139 & 127 & 133 & 118 & 139 & 122 & 138 & 121 & 129 \\
\hline $10: 31: \odot \odot$ & 631 & 139 & 127 & 133 & 118 & 139 & 122 & 138 & 121 & 129 \\
\hline $10: 32: \odot \odot$ & 632 & 139 & 127 & 133 & 118 & 139 & 122 & 138 & 121 & 129 \\
\hline $10: 33: \odot \odot$ & 633 & 139 & 127 & 133 & 118 & 139 & 122 & 138 & 121 & 129 \\
\hline $10: 34: \odot \odot$ & 634 & 139 & 127 & 133 & 118 & 139 & 122 & 138 & 121 & 129 \\
\hline $10: 35: \odot \odot$ & 635 & 139 & 127 & 133 & 118 & 139 & 122 & 138 & 121 & 129 \\
\hline $10: 36: \odot \odot$ & 636 & 139 & 127 & 133 & 118 & 139 & 122 & 138 & 121 & 129 \\
\hline $10: 37: \odot \odot$ & 637 & 139 & 127 & 133 & 118 & 139 & 122 & 138 & 121 & 129 \\
\hline $10: 38: \odot \odot$ & 638 & 139 & 127 & 133 & 119 & 139 & 122 & 138 & 121 & 130 \\
\hline $10: 39: \odot \odot$ & 639 & 139 & 127 & 133 & 119 & 139 & 122 & 138 & 121 & 130 \\
\hline $10: 40: \odot \odot$ & 640 & 139 & 127 & 133 & 119 & 139 & 122 & 138 & 121 & 130 \\
\hline $10: 41: 0 \odot$ & 641 & 139 & 127 & 133 & 119 & 139 & 122 & 138 & 121 & 130 \\
\hline $10: 42: \odot \odot$ & 642 & 139 & 127 & 133 & 119 & 139 & 122 & 138 & 121 & 130 \\
\hline $10: 43: \odot \odot$ & 643 & 139 & 127 & 133 & 119 & 139 & 122 & 138 & 121 & 130 \\
\hline $10: 44: \odot \odot$ & 644 & 139 & 127 & 133 & 119 & 139 & 122 & 138 & 121 & 130 \\
\hline $10: 45: \odot \odot$ & 645 & 139 & 127 & 133 & 119 & 139 & 122 & 138 & 121 & 130 \\
\hline $10: 46: \odot \odot$ & 646 & 138 & 127 & 133 & 119 & 139 & 122 & 138 & 121 & 129 \\
\hline $10: 47: \odot \odot$ & 647 & 138 & 127 & 133 & 119 & 139 & 122 & 138 & 121 & 129 \\
\hline $10: 48: \odot \odot$ & 648 & 138 & 127 & 133 & 119 & 139 & 122 & 138 & 122 & 130 \\
\hline $10: 49: \odot \odot$ & 649 & 138 & 127 & 133 & 119 & 138 & 122 & 138 & 122 & 129 \\
\hline 1०: $50: \odot \odot$ & 650 & 138 & 127 & 133 & 119 & 138 & 122 & 138 & 122 & 129 \\
\hline $10: 51: 00$ & 651 & 138 & 127 & 133 & 119 & 138 & 122 & 138 & 122 & 129 \\
\hline $10: 52: \odot \odot$ & 652 & 138 & 127 & 133 & 119 & 138 & 122 & 138 & 122 & 129 \\
\hline $10: 53: \odot \odot$ & 653 & 138 & 127 & 133 & 119 & 138 & 122 & 137 & 122 & 129 \\
\hline $10: 54: \odot \odot$ & 654 & 138 & 127 & 133 & 119 & 138 & 122 & 137 & 122 & 129 \\
\hline $10: 55: \odot \odot$ & 655 & 138 & 127 & 133 & 119 & 138 & 122 & 137 & 122 & 129 \\
\hline $10: 56: \odot \odot$ & 656 & 138 & 127 & 133 & 119 & 138 & 122 & 137 & 122 & 129 \\
\hline $10: 57: \odot \odot$ & 657 & 138 & 127 & 133 & 119 & 138 & 122 & 137 & 122 & 129 \\
\hline $10: 58: \odot \odot$ & 658 & 137 & 127 & 133 & 119 & 138 & 122 & 137 & 122 & 129 \\
\hline $10: 59: \odot \odot$ & 659 & 137 & 126 & 133 & 118 & 137 & 122 & 137 & 121 & 129 \\
\hline 11: ๑९: ๑९ & 660 & 137 & 126 & 132 & 118 & 137 & 121 & 136 & 121 & 128 \\
\hline 11: $01: \odot \odot$ & 661 & 137 & 126 & 132 & 118 & 137 & 121 & 136 & 121 & 128 \\
\hline $11: \odot 2: \odot \odot$ & 662 & 137 & 126 & 132 & 118 & 137 & 121 & 136 & 121 & 128 \\
\hline 11: ๑3:๑๑ & 663 & 137 & 126 & 132 & 118 & 137 & 121 & 136 & 121 & 128 \\
\hline
\end{tabular}




\begin{tabular}{|c|c|c|c|c|c|c|c|c|c|c|}
\hline $11: \odot 4: \odot \odot$ & 664 & 137 & 126 & 132 & 118 & 137 & 121 & 136 & 121 & 128 \\
\hline $11: 05: 00$ & 665 & 137 & 126 & 132 & 118 & 136 & 121 & 136 & 121 & 128 \\
\hline $11: \odot 6: \odot \odot$ & 666 & 136 & 126 & 132 & 118 & 136 & 121 & 136 & 121 & 128 \\
\hline $11: \odot 7: \odot \odot$ & 667 & 136 & 126 & 132 & 118 & 136 & 121 & 136 & 121 & 128 \\
\hline 11: ๑8: ๑९ & 668 & 136 & 126 & 132 & 118 & 136 & 121 & 136 & 121 & 128 \\
\hline 11: ๑9:๑๑ & 669 & 136 & 125 & 132 & 118 & 136 & 121 & 135 & 121 & 128 \\
\hline $11: 10: \odot \odot$ & 670 & 136 & 125 & 132 & 117 & 136 & 121 & 135 & 121 & 128 \\
\hline $11: 11: 0 \odot$ & 671 & 136 & 125 & 132 & 117 & 136 & 121 & 135 & 121 & 128 \\
\hline $11: 12: \odot \odot$ & 672 & 136 & 125 & 132 & 117 & 136 & 119 & 135 & 121 & 127 \\
\hline $11: 13: \odot \odot$ & 673 & 136 & 125 & 130 & 117 & 136 & 119 & 135 & 121 & 127 \\
\hline $11: 14: \odot \odot$ & 674 & 136 & 125 & 130 & 117 & 135 & 119 & 135 & 121 & 127 \\
\hline $11: 15: \odot \odot$ & 675 & 135 & 125 & 130 & 117 & 135 & 119 & 135 & 121 & 127 \\
\hline $11: 16: \odot \odot$ & 676 & 135 & 125 & 130 & 117 & 135 & 119 & 135 & 121 & 127 \\
\hline $11: 17: \odot \odot$ & 677 & 135 & 125 & 130 & 117 & 135 & 119 & 135 & 119 & 127 \\
\hline $11: 18: \odot \odot$ & 678 & 135 & 125 & 130 & 117 & 135 & 119 & 135 & 119 & 127 \\
\hline $11: 19: \odot \odot$ & 679 & 135 & 124 & 130 & 117 & 135 & 119 & 134 & 119 & 127 \\
\hline $11: 20: \odot \odot$ & 680 & 135 & 124 & 130 & 117 & 135 & 119 & 134 & 119 & 127 \\
\hline $11: 21: \odot \odot$ & 681 & 135 & 124 & 130 & 117 & 134 & 119 & 134 & 119 & 127 \\
\hline $11: 22: \odot \odot$ & 682 & 135 & 124 & 130 & 117 & 134 & 119 & 134 & 119 & 127 \\
\hline $11: 23: \odot \odot$ & 683 & 135 & 124 & 130 & 117 & 134 & 119 & 134 & 119 & 127 \\
\hline $11: 24: \odot \odot$ & 684 & 135 & 124 & 130 & 116 & 134 & 118 & 134 & 119 & 126 \\
\hline $11: 25: 0 \odot$ & 685 & 135 & 124 & 130 & 116 & 134 & 118 & 134 & 119 & 126 \\
\hline $11: 26: \odot \odot$ & 686 & 134 & 124 & 130 & 116 & 134 & 118 & 134 & 119 & 126 \\
\hline $11: 27: \odot \odot$ & 687 & 134 & 124 & 130 & 116 & 134 & 118 & 134 & 119 & 126 \\
\hline $11: 28: \odot \odot$ & 688 & 134 & 124 & 129 & 116 & 134 & 118 & 133 & 119 & 126 \\
\hline $11: 29: \odot \odot$ & 689 & 134 & 123 & 129 & 116 & 134 & 118 & 133 & 119 & 126 \\
\hline $11: 30: \odot \odot$ & 690 & 134 & 123 & 129 & 116 & 134 & 118 & 133 & 119 & 126 \\
\hline $11: 31: 0 \odot$ & 691 & 134 & 123 & 129 & 116 & 134 & 118 & 133 & 118 & 126 \\
\hline $11: 32: \odot \odot$ & 692 & 134 & 123 & 129 & 116 & 134 & 118 & 133 & 118 & 126 \\
\hline $11: 33: \odot \odot$ & 693 & 134 & 123 & 129 & 116 & 132 & 118 & 133 & 118 & 125 \\
\hline $11: 34: \odot \odot$ & 694 & 134 & 123 & 129 & 116 & 132 & 118 & 133 & 118 & 125 \\
\hline $11: 35: \odot \odot$ & 695 & 134 & 123 & 129 & 116 & 132 & 117 & 133 & 118 & 125 \\
\hline $11: 36: \odot \odot$ & 696 & 134 & 123 & 129 & 116 & 132 & 117 & 133 & 118 & 125 \\
\hline $11: 37: \odot \odot$ & 697 & 133 & 123 & 129 & 116 & 132 & 117 & 133 & 118 & 125 \\
\hline $11: 38: 0 \odot$ & 698 & 133 & 123 & 129 & 116 & 132 & 117 & 133 & 118 & 125 \\
\hline $11: 39: \odot \odot$ & 699 & 133 & 123 & 129 & 116 & 132 & 117 & 133 & 118 & 125 \\
\hline $11: 40: \odot \odot$ & 700 & 133 & 123 & 129 & 116 & 132 & 117 & 133 & 118 & 125 \\
\hline $11: 41: \odot \odot$ & 701 & 133 & 123 & 129 & 116 & 132 & 117 & 132 & 118 & 125 \\
\hline $11: 42: \odot \odot$ & 702 & 133 & 123 & 129 & 116 & 132 & 117 & 132 & 118 & 125 \\
\hline $11: 43: \odot \odot$ & 703 & 133 & 123 & 129 & 116 & 132 & 117 & 132 & 118 & 125 \\
\hline $11: 44: \odot \odot$ & $7 \odot 4$ & 133 & 123 & 129 & 116 & 132 & 117 & 132 & 118 & 125 \\
\hline $11: 45: \odot \odot$ & 705 & 133 & 123 & 129 & 114 & 131 & 117 & 132 & 118 & 125 \\
\hline $11: 46: \odot \odot$ & 706 & 132 & 121 & 128 & 114 & 131 & 116 & 132 & 118 & 124 \\
\hline $11: 47: \odot \odot$ & 707 & 132 & 121 & 128 & 114 & 131 & 116 & 132 & 118 & 124 \\
\hline $11: 48: \odot \odot$ & 708 & 132 & 121 & 128 & 114 & 131 & 116 & 132 & 118 & 124 \\
\hline $11: 49: \odot \odot$ & 709 & 132 & 121 & 128 & 114 & 131 & 116 & 132 & 118 & 124 \\
\hline $11: 50: \odot \odot$ & 710 & 132 & 121 & 128 & 114 & 131 & 116 & 132 & 118 & 124 \\
\hline $11: 51: \odot \odot$ & 711 & 132 & 121 & 128 & 114 & 131 & 116 & 131 & 117 & 124 \\
\hline $11: 52: \odot \odot$ & 712 & 132 & 121 & 128 & 114 & 131 & 116 & 131 & 117 & 124 \\
\hline $11: 53: \odot \odot$ & 713 & 132 & 121 & 128 & 114 & 131 & 116 & 131 & 117 & 124 \\
\hline $11: 54: \odot \odot$ & 714 & 132 & 121 & 128 & 114 & 131 & 116 & 131 & 117 & 124 \\
\hline $11: 55: \odot \odot$ & 715 & 132 & 121 & 128 & 114 & 130 & 116 & 131 & 117 & 124 \\
\hline $11: 56: \odot \odot$ & 716 & 132 & 121 & 128 & 114 & 130 & 116 & 131 & 117 & 124 \\
\hline $11: 57: \odot \odot$ & 717 & 132 & 121 & 128 & 114 & 130 & 116 & 131 & 117 & 124 \\
\hline $11: 58: \odot \odot$ & 718 & 132 & 120 & 128 & 114 & 130 & 116 & 131 & 117 & 124 \\
\hline $11: 59: \odot \odot$ & 719 & 130 & 120 & 128 & 114 & 130 & 115 & 130 & 116 & 123 \\
\hline 12: $\bullet \odot: \odot \odot$ & 720 & 130 & 120 & 128 & 114 & 130 & 115 & 130 & 116 & 123 \\
\hline
\end{tabular}




\begin{tabular}{|c|c|c|c|c|c|c|c|c|c|c|}
\hline $12: 01: \odot \odot$ & 721 & 130 & 120 & 128 & 114 & 130 & 115 & 130 & 116 & 123 \\
\hline $12: \odot 2: \odot \odot$ & 722 & 130 & 120 & 128 & 114 & 130 & 115 & 130 & 116 & 123 \\
\hline $12: \odot 3: \odot \odot$ & 723 & 130 & 120 & 128 & 114 & 130 & 115 & 130 & 116 & 123 \\
\hline $12: \odot 4: \odot \odot$ & 724 & 130 & 120 & 128 & 114 & 130 & 115 & 130 & 116 & 123 \\
\hline $12: \odot 5: \odot \odot$ & 725 & 130 & 120 & 127 & 114 & 130 & 115 & 130 & 116 & 123 \\
\hline $12: \odot 6: \odot \odot$ & 726 & 130 & 120 & 127 & 114 & 130 & 115 & 130 & 116 & 123 \\
\hline $12: \odot 7: \odot \odot$ & 727 & 130 & 120 & 127 & 114 & 130 & 115 & 130 & 116 & 123 \\
\hline 12: $: 8: \odot \odot$ & 728 & 130 & 119 & 127 & 114 & 129 & 115 & 130 & 116 & 123 \\
\hline 12:๑9:๑๑ & 729 & 130 & 119 & 127 & 114 & 129 & 115 & 130 & 116 & 123 \\
\hline $12: 10: \odot \odot$ & 730 & 130 & 119 & 127 & 114 & 129 & 115 & 130 & 116 & 123 \\
\hline $12: 11: \odot \odot$ & 731 & 129 & 119 & 127 & 114 & 129 & 114 & 130 & 116 & 122 \\
\hline $12: 12: \odot \odot$ & 732 & 129 & 119 & 127 & 114 & 129 & 114 & 130 & 116 & 122 \\
\hline $12: 13: \odot \odot$ & 733 & 129 & 119 & 127 & 114 & 129 & 114 & 130 & 116 & 122 \\
\hline $12: 14: \odot \odot$ & 734 & 129 & 119 & 127 & 114 & 129 & 114 & 129 & 116 & 122 \\
\hline $12: 15: \odot \odot$ & 735 & 129 & 119 & 127 & 114 & 129 & 114 & 129 & 116 & 122 \\
\hline $12: 16: \odot \odot$ & 736 & 129 & 119 & 126 & 113 & 129 & 114 & 129 & 115 & 122 \\
\hline $12: 17: \odot \odot$ & 737 & 129 & 119 & 126 & 113 & 129 & 114 & 129 & 115 & 122 \\
\hline $12: 18: \odot \odot$ & 738 & 129 & 119 & 126 & 113 & 129 & 114 & 129 & 115 & 122 \\
\hline $12: 19: \odot \odot$ & 739 & 129 & 119 & 126 & 113 & 128 & 114 & 129 & 115 & 122 \\
\hline $12: 2 \odot: \odot \odot$ & 740 & 129 & 119 & 126 & 113 & 128 & 114 & 129 & 115 & 122 \\
\hline $12: 21: \odot \odot$ & 741 & 129 & 119 & 126 & 113 & 128 & 114 & 129 & 115 & 122 \\
\hline $12: 22: \odot \odot$ & 742 & 129 & 118 & 126 & 113 & 128 & 114 & 129 & 115 & 122 \\
\hline $12: 23: \odot \odot$ & 743 & 128 & 118 & 126 & 113 & 128 & 114 & 129 & 115 & 121 \\
\hline $12: 24: \odot \odot$ & 744 & 128 & 118 & 126 & 113 & 128 & 114 & 129 & 115 & 121 \\
\hline $12: 25: \odot \odot$ & 745 & 128 & 118 & 126 & 113 & 128 & 114 & 129 & 115 & 121 \\
\hline $12: 26: \odot \odot$ & 746 & 128 & 118 & 126 & 113 & 128 & 114 & 128 & 115 & 121 \\
\hline $12: 27: \odot \odot$ & 747 & 128 & 118 & 126 & 113 & 128 & 114 & 128 & 114 & 121 \\
\hline $12: 28: \odot \odot$ & 748 & 128 & 118 & 126 & 113 & 128 & 114 & 128 & 114 & 121 \\
\hline $12: 29: \odot \odot$ & 749 & 128 & 118 & 126 & 113 & 127 & 114 & 128 & 114 & 121 \\
\hline $12: 3 \odot: \odot \odot$ & 750 & 128 & 118 & 126 & 112 & 127 & 114 & 128 & 114 & 121 \\
\hline $12: 31: \odot \odot$ & 751 & 128 & 118 & 126 & 112 & 127 & 114 & 128 & 114 & 121 \\
\hline $12: 32: \odot \odot$ & 752 & 128 & 118 & 125 & 112 & 127 & 114 & 128 & 114 & 121 \\
\hline $12: 33: \odot \odot$ & 753 & 128 & 118 & 125 & 112 & 127 & 114 & 128 & 114 & 121 \\
\hline $12: 34: 0 \odot$ & 754 & 128 & 118 & 125 & 112 & 127 & 114 & 128 & 114 & 121 \\
\hline $12: 35: \odot \odot$ & 755 & 128 & 118 & 125 & 112 & 127 & 114 & 128 & 114 & 121 \\
\hline $12: 36: \odot \odot$ & 756 & 127 & 117 & 125 & 112 & 127 & 114 & 127 & 114 & 120 \\
\hline $12: 37: \odot \odot$ & 757 & 127 & 117 & 125 & 112 & 127 & 114 & 127 & 114 & 120 \\
\hline $12: 38: \odot \odot$ & 758 & 127 & 117 & 125 & 112 & 126 & 114 & 127 & 114 & 120 \\
\hline $12: 39: \odot \odot$ & 759 & 127 & 117 & 125 & 112 & 126 & 114 & 127 & 114 & 120 \\
\hline $12: 4 \odot: \odot \odot$ & 760 & 127 & 117 & 125 & 112 & 126 & 114 & 127 & 114 & 120 \\
\hline $12: 41: \odot \odot$ & 761 & 127 & 117 & 125 & 112 & 126 & 114 & 127 & 114 & 120 \\
\hline $12: 42: \odot \odot$ & 762 & 127 & 117 & 125 & 112 & 126 & 114 & 127 & 114 & 120 \\
\hline $12: 43: \odot \odot$ & 763 & 127 & 117 & 125 & 112 & 126 & 114 & 127 & 114 & 120 \\
\hline $12: 44: \odot \odot$ & 764 & 127 & 117 & 125 & 112 & 126 & 113 & 127 & 113 & 120 \\
\hline $12: 45: \odot \odot$ & 765 & 127 & 117 & 125 & 111 & 126 & 113 & 127 & 113 & 120 \\
\hline $12: 46: \odot \odot$ & 766 & 127 & 117 & 125 & 111 & 126 & 113 & 127 & 113 & 120 \\
\hline $12: 47: \odot \odot$ & 767 & 127 & 117 & 125 & 111 & 126 & 113 & 127 & 113 & 120 \\
\hline $12: 48: \odot \odot$ & 768 & 127 & 117 & 125 & 111 & 126 & 113 & 127 & 113 & 120 \\
\hline $12: 49: \odot \odot$ & 769 & 127 & 117 & 124 & 111 & 126 & 113 & 125 & 113 & 120 \\
\hline $12: 5 \odot: \odot \odot$ & 770 & 127 & 117 & 124 & 111 & 126 & 113 & 125 & 113 & 120 \\
\hline $12: 51: \odot \odot$ & 771 & 127 & 117 & 124 & 111 & 125 & 113 & 125 & 113 & 119 \\
\hline $12: 52: \odot \odot$ & 772 & 126 & 117 & 124 & 111 & 125 & 113 & 125 & 113 & 119 \\
\hline $12: 53: \odot \odot$ & 773 & 126 & 116 & 124 & 111 & 125 & 113 & 125 & 113 & 119 \\
\hline $12: 54: \odot \odot$ & 774 & 126 & 116 & 124 & 111 & 125 & 113 & 125 & 113 & 119 \\
\hline $12: 55: \odot \odot$ & 775 & 126 & 116 & 124 & 111 & 125 & 113 & 125 & 113 & 119 \\
\hline $12: 56: \odot \odot$ & 776 & 126 & 116 & 124 & 111 & 125 & 113 & 125 & 113 & 119 \\
\hline $12: 57: \odot \odot$ & 777 & 126 & 116 & 124 & 111 & 125 & 112 & 125 & 113 & 119 \\
\hline
\end{tabular}




\begin{tabular}{|c|c|c|c|c|c|c|c|c|c|c|}
\hline $12: 58: \odot \odot$ & 778 & 126 & 116 & 124 & 111 & 125 & 112 & 125 & 113 & 119 \\
\hline $12: 59: \odot \odot$ & 779 & 126 & 116 & 124 & 111 & 125 & 112 & 125 & 112 & 119 \\
\hline 13: ๑९:๑९ & 780 & 125 & 116 & 124 & 111 & 125 & 112 & 124 & 112 & 119 \\
\hline $13: \odot 1: \odot \odot$ & 781 & 125 & 116 & 124 & 111 & 125 & 112 & 124 & 112 & 119 \\
\hline $13: \odot 2: \odot \odot$ & 782 & 125 & 116 & 123 & 111 & 125 & 112 & 124 & 112 & 118 \\
\hline $13: \odot 3: \odot \odot$ & 783 & 125 & 115 & 123 & 111 & 124 & 112 & 124 & 112 & 118 \\
\hline $13: \odot 4: \odot \odot$ & 784 & 125 & 115 & 123 & 111 & 124 & 112 & 124 & 112 & 118 \\
\hline 13: ९5:๑९ & 785 & 125 & 115 & 123 & 111 & 124 & 112 & 124 & 112 & 118 \\
\hline $13: \odot 6: \odot \odot$ & 786 & 125 & 115 & 123 & 111 & 124 & 112 & 124 & 112 & 118 \\
\hline $13: \odot 7: \odot \odot$ & 787 & 125 & 115 & 123 & 111 & 124 & 112 & 124 & 112 & 118 \\
\hline 13:०৪:०९ & 788 & 125 & 115 & 123 & 111 & 124 & 111 & 123 & 112 & 118 \\
\hline 13:๑9:๑९ & 789 & 124 & 115 & 123 & 110 & 124 & 111 & 123 & 112 & 118 \\
\hline $13: 10: \odot \odot$ & 790 & 124 & 115 & 123 & 110 & 124 & 111 & 123 & 112 & 118 \\
\hline $13: 11: \odot \odot$ & 791 & 124 & 115 & 123 & 110 & 124 & 111 & 123 & 112 & 118 \\
\hline $13: 12: \odot \odot$ & 792 & 124 & 115 & 123 & 110 & 124 & 111 & 123 & 112 & 118 \\
\hline $13: 13: \odot \odot$ & 793 & 124 & 115 & 123 & 110 & 124 & 111 & 123 & 112 & 118 \\
\hline $13: 14: \odot \odot$ & 794 & 124 & 115 & 123 & 110 & 124 & 111 & 123 & 111 & 118 \\
\hline $13: 15: \odot \odot$ & 795 & 124 & 115 & 123 & 110 & 124 & 111 & 123 & 111 & 118 \\
\hline $13: 16: \odot \odot$ & 796 & 124 & 115 & 123 & 110 & 123 & 111 & 123 & 111 & 117 \\
\hline $13: 17: \odot \odot$ & 797 & 124 & 115 & 122 & 110 & 123 & 111 & 123 & 111 & 117 \\
\hline $13: 18: \odot \odot$ & 798 & 124 & 115 & 122 & 110 & 123 & 111 & 123 & 111 & 117 \\
\hline $13: 19: \odot \odot$ & 799 & 124 & 114 & 122 & 110 & 123 & 111 & 123 & 111 & 117 \\
\hline $13: 20: \odot \odot$ & 800 & 124 & 114 & 122 & 110 & 123 & 111 & 123 & 111 & 117 \\
\hline $13: 21: \odot \odot$ & 801 & 124 & 114 & 122 & 110 & 123 & 111 & 123 & 111 & 117 \\
\hline $13: 22: 0 \odot$ & 802 & 124 & 114 & 122 & 110 & 123 & 111 & 122 & 111 & 117 \\
\hline $13: 23: \odot \odot$ & 803 & 124 & 114 & 122 & 110 & 123 & 111 & 122 & 111 & 117 \\
\hline $13: 24: 0 \odot$ & 804 & 124 & 114 & 122 & 110 & 123 & 111 & 122 & 111 & 117 \\
\hline $13: 25: \odot \odot$ & 805 & 124 & 114 & 122 & 110 & 123 & 111 & 122 & 111 & 117 \\
\hline $13: 26: \odot \odot$ & 806 & 124 & 114 & 122 & 110 & 122 & 111 & 122 & 111 & 117 \\
\hline $13: 27: \odot \odot$ & 807 & 124 & 114 & 122 & 110 & 122 & 111 & 122 & 111 & 117 \\
\hline $13: 28: \odot \odot$ & 808 & 123 & 114 & 122 & 109 & 122 & 111 & 122 & 111 & 117 \\
\hline $13: 29: \odot \odot$ & 809 & 123 & 114 & 122 & 109 & 122 & 111 & 122 & 110 & 116 \\
\hline $13: 3 \odot: \odot \odot$ & 810 & 123 & 114 & 122 & 109 & 122 & 111 & 122 & 110 & 116 \\
\hline $13: 31: 0 \odot$ & 811 & 123 & 114 & 122 & 109 & 122 & 111 & 122 & 110 & 116 \\
\hline $13: 32: \odot \odot$ & 812 & 123 & 114 & 122 & 109 & 122 & 111 & 122 & 110 & 116 \\
\hline $13: 33: \odot \odot$ & 813 & 123 & 113 & 122 & 109 & 122 & 111 & 122 & 110 & 116 \\
\hline $13: 34: \odot \odot$ & 814 & 123 & 113 & 122 & 109 & 122 & 111 & 122 & 110 & 116 \\
\hline $13: 35: 0 \odot$ & 815 & 123 & 113 & 122 & 109 & 122 & 111 & 122 & 110 & 116 \\
\hline $13: 36: \odot \odot$ & 816 & 123 & 113 & 122 & 109 & 122 & 110 & 122 & 110 & 116 \\
\hline $13: 37: \odot \odot$ & 817 & 123 & 113 & 122 & 109 & 122 & 110 & 122 & 110 & 116 \\
\hline $13: 38: \odot \odot$ & 818 & 123 & 113 & 121 & 109 & 122 & 110 & 122 & 110 & 116 \\
\hline $13: 39: \odot \odot$ & 819 & 123 & 113 & 121 & 109 & 122 & 110 & 122 & 110 & 116 \\
\hline $13: 4 \odot: \odot \odot$ & 820 & 123 & 113 & 121 & 109 & 122 & 110 & 121 & 110 & 116 \\
\hline $13: 41: \odot \odot$ & 821 & 123 & 113 & 121 & 109 & 122 & 110 & 121 & 110 & 116 \\
\hline $13: 42: \odot \odot$ & 822 & 123 & 113 & 121 & 109 & 122 & 110 & 121 & 110 & 116 \\
\hline $13: 43: \odot \odot$ & 823 & 122 & 113 & 121 & 109 & 121 & 110 & 121 & 110 & 116 \\
\hline $13: 48: \odot \odot$ & 828 & 122 & 113 & 121 & 108 & 121 & 110 & 121 & 109 & 115 \\
\hline $13: 53: \odot \odot$ & 833 & 121 & 112 & 120 & 108 & 121 & 109 & 120 & 109 & 115 \\
\hline $13: 58: \odot \odot$ & 838 & 121 & 112 & 120 & 108 & 121 & 107 & 120 & 109 & 115 \\
\hline $14: \odot 3: \odot \odot$ & 843 & 119 & 112 & 119 & 108 & 119 & 106 & 119 & 108 & 114 \\
\hline $14: \odot 8: \odot \odot$ & 848 & 119 & 112 & 119 & 108 & 119 & 106 & 119 & 108 & 114 \\
\hline $14: 13: \odot \odot$ & 853 & 118 & 111 & 119 & 107 & 118 & 105 & 118 & 107 & 113 \\
\hline $14: 18: \odot \odot$ & 858 & 118 & 111 & 118 & 107 & 118 & 104 & 118 & 107 & 113 \\
\hline $14: 23: \odot \odot$ & 863 & 118 & 110 & 118 & 107 & 118 & 104 & 118 & 107 & 112 \\
\hline $14: 28: \odot \odot$ & 868 & 118 & 110 & 118 & 106 & 117 & 104 & 117 & 107 & 112 \\
\hline $14: 33: \odot \odot$ & 873 & 117 & 110 & 118 & 106 & 117 & 104 & 117 & 105 & 112 \\
\hline $14: 38: \odot \odot$ & 878 & 117 & 109 & 116 & 106 & 116 & 105 & 117 & 105 & 111 \\
\hline
\end{tabular}




\begin{tabular}{|c|c|c|c|c|c|c|c|c|c|c|}
\hline $14: 43: \odot \odot$ & 883 & 117 & 109 & 116 & 105 & 116 & 105 & 116 & 105 & 111 \\
\hline $14: 48: \odot \odot$ & 888 & 117 & 109 & 116 & 105 & 116 & 105 & 116 & 105 & 111 \\
\hline $14: 53: \odot \odot$ & 893 & 116 & 107 & 116 & 105 & 116 & 105 & 116 & 105 & 111 \\
\hline $14: 58: \odot \odot$ & 898 & 116 & 107 & 115 & 104 & 115 & 105 & 116 & 104 & 110 \\
\hline $15: \odot 3: \odot \odot$ & 903 & 116 & 107 & 115 & 104 & 115 & 105 & 115 & 104 & 110 \\
\hline $15: \odot 8: \odot \odot$ & 908 & 115 & 107 & 115 & 104 & 115 & 105 & 115 & 104 & 110 \\
\hline $15: 13: \odot \odot$ & 913 & 115 & 106 & 114 & 104 & 114 & 104 & 115 & 104 & 110 \\
\hline $15: 18: \odot \odot$ & 918 & 115 & 106 & 114 & 104 & 114 & 104 & 114 & 103 & 109 \\
\hline $15: 23: \odot \odot$ & 923 & 114 & 106 & 114 & 103 & 113 & 104 & 114 & 103 & 109 \\
\hline $15: 28: \odot \odot$ & 928 & 114 & 105 & 113 & 103 & 113 & 104 & 114 & 103 & 109 \\
\hline $15: 33: \odot \odot$ & 933 & 114 & 105 & 113 & 103 & 113 & 104 & 114 & 103 & 109 \\
\hline $15: 38: \odot \odot$ & 938 & 114 & 105 & 113 & 103 & 113 & 104 & 112 & 102 & 108 \\
\hline $15: 43: \odot \odot$ & 943 & 113 & 105 & 113 & 103 & 112 & 103 & 112 & 102 & 108 \\
\hline $15: 48: \odot \odot$ & 948 & 113 & 104 & 112 & 101 & 112 & 103 & 111 & 102 & 107 \\
\hline $15: 53: \odot \odot$ & 953 & 112 & 104 & 112 & 101 & 111 & 103 & 111 & 102 & 107 \\
\hline $15: 58: \odot \odot$ & 958 & 112 & 103 & 111 & 101 & 111 & 102 & 110 & 101 & 106 \\
\hline $16: \odot 3: \odot \odot$ & 963 & 112 & 103 & 111 & 101 & 111 & 102 & 110 & 101 & 106 \\
\hline 16: ๑8:๑९ & 968 & 111 & 103 & 110 & 100 & 109 & 102 & 109 & 101 & 106 \\
\hline $16: 13: \odot \odot$ & 973 & 111 & 102 & 110 & 100 & 109 & 101 & 109 & 100 & 105 \\
\hline $16: 18: \odot \odot$ & 978 & 111 & 102 & 110 & 100 & 109 & 101 & 108 & 100 & 105 \\
\hline $16: 23: \odot \odot$ & 983 & 110 & 102 & 109 & 100 & 108 & 100 & 108 & 100 & 105 \\
\hline $16: 28: \odot \odot$ & 988 & 110 & 101 & 109 & 100 & 108 & 100 & 107 & 99 & 104 \\
\hline $16: 33: \odot \odot$ & 993 & 109 & 101 & 109 & 100 & 108 & 100 & 107 & 99 & 104 \\
\hline $16: 38: \odot \odot$ & 998 & 109 & 101 & 108 & 99 & 107 & 100 & 107 & 99 & 104 \\
\hline $16: 43: \odot \odot$ & 1003 & 109 & 101 & 108 & 99 & 107 & 99 & 107 & 98 & 103 \\
\hline $16: 48: \odot \odot$ & 1008 & 108 & 100 & 108 & 99 & 107 & 99 & 106 & 98 & 103 \\
\hline $16: 53: \odot \odot$ & 1013 & 108 & 100 & 107 & 99 & 106 & 99 & 106 & 98 & 103 \\
\hline $16: 58: 0 \odot$ & 1018 & 108 & 100 & 107 & 98 & 106 & 99 & 105 & 98 & 102 \\
\hline $17: \odot 3: \odot \odot$ & 1023 & 108 & 100 & 107 & 98 & 106 & 99 & 105 & 98 & 102 \\
\hline $17: \odot 8: \odot \odot$ & 1028 & 108 & 100 & 107 & 98 & 106 & 99 & 105 & 97 & 102 \\
\hline $17: 13: \odot \odot$ & 1033 & 107 & 100 & 107 & 98 & 106 & 99 & 105 & 97 & 102 \\
\hline $17: 18: \odot \odot$ & 1038 & 107 & 100 & 107 & 98 & 105 & 99 & 105 & 97 & 102 \\
\hline $17: 23: \odot \odot$ & 1043 & 107 & 99 & 107 & 98 & 105 & 99 & 105 & 97 & 102 \\
\hline $17: 28: 0 \odot$ & 1048 & 107 & 99 & 107 & 98 & 105 & 99 & 105 & 97 & 102 \\
\hline $17: 33: \odot \odot$ & 1053 & 107 & 99 & 107 & 98 & 105 & 98 & 105 & 97 & 102 \\
\hline $17: 38: \odot \odot$ & 1058 & 107 & 99 & 107 & 98 & 105 & 98 & 105 & 97 & 102 \\
\hline $17: 43: \odot \odot$ & 1063 & 107 & 99 & 107 & 97 & 105 & 98 & 105 & 97 & 102 \\
\hline $17: 48: \odot \odot$ & 1068 & 107 & 99 & 106 & 97 & 105 & 98 & 105 & 97 & 102 \\
\hline $17: 53: \odot \odot$ & 1073 & 107 & 99 & 106 & 97 & 105 & 98 & 105 & 96 & 101 \\
\hline $17: 58: \odot \odot$ & 1078 & 105 & 99 & 106 & 97 & 105 & 98 & 105 & 96 & 101 \\
\hline 18: ๑3: ๑९ & 1083 & 105 & 99 & 106 & 97 & 104 & 98 & 105 & 96 & 101 \\
\hline 18: ๑8:๑९ & 1088 & 105 & 99 & 106 & 97 & 104 & 98 & 105 & 96 & 101 \\
\hline $18: 13: \odot \odot$ & 1093 & 105 & 98 & 106 & 97 & 104 & 97 & 104 & 96 & 101 \\
\hline $18: 18: \odot \odot$ & 1098 & 105 & 98 & 106 & 97 & 104 & 97 & 104 & 96 & 101 \\
\hline $18: 23: \odot \odot$ & 1103 & 105 & 98 & 106 & 97 & 104 & 97 & 104 & 96 & 101 \\
\hline $18: 28: \odot \odot$ & 1108 & 105 & 98 & 105 & 96 & 104 & 97 & 104 & 96 & 100 \\
\hline $18: 33: \odot \odot$ & 1113 & 104 & 98 & 105 & 96 & 103 & 97 & 104 & 96 & 100 \\
\hline 18: $38: \odot \odot$ & 1118 & 104 & 98 & 105 & 96 & 103 & 96 & 104 & 96 & 100 \\
\hline $18: 43: \odot \odot$ & 1123 & 104 & 96 & 103 & 96 & 103 & 96 & 103 & 95 & 99 \\
\hline $18: 48: \odot \odot$ & 1128 & 103 & 96 & 103 & 96 & 102 & 94 & 103 & 95 & 99 \\
\hline $18: 53: 00$ & 1133 & 103 & 96 & 103 & 95 & 102 & 94 & 103 & 95 & 99 \\
\hline $18: 58: \odot \odot$ & 1138 & 103 & 96 & 103 & 95 & 102 & 94 & 102 & 94 & 99 \\
\hline 19:๑3:๑๑ & 1143 & 103 & 96 & 103 & 95 & 102 & 94 & 102 & 94 & 99 \\
\hline 19: ๑৪:०९ & 1148 & 103 & 96 & 103 & 95 & 102 & 94 & 102 & 94 & 99 \\
\hline $19: 13: \odot \odot$ & 1153 & 103 & 96 & 103 & 95 & 102 & 94 & 102 & 94 & 99 \\
\hline $19: 18: \odot \odot$ & 1158 & 102 & 95 & 102 & 95 & 101 & 94 & 102 & 94 & 98 \\
\hline $19: 23: \odot \odot$ & 1163 & 102 & 95 & 102 & 95 & 101 & 94 & 102 & 94 & 98 \\
\hline
\end{tabular}




\begin{tabular}{|c|c|c|c|c|c|c|c|c|c|c|}
\hline $19: 28: \odot \odot$ & 1168 & 102 & 95 & 102 & 95 & 101 & 94 & 102 & 94 & 98 \\
\hline $19: 33: \odot \odot$ & 1173 & 102 & 95 & 102 & 95 & 101 & 94 & 102 & 94 & 98 \\
\hline $19: 38: \odot \odot$ & 1178 & 102 & 95 & 101 & 94 & 101 & 94 & 101 & 94 & 98 \\
\hline $19: 43: \odot \odot$ & 1183 & 102 & 95 & 101 & 94 & 101 & 94 & 101 & 94 & 98 \\
\hline $19: 48: \odot \odot$ & 1188 & 101 & 94 & 101 & 94 & 100 & 94 & 101 & 92 & 97 \\
\hline $19: 53: \odot \odot$ & 1193 & 101 & 94 & 101 & 94 & 100 & 93 & 101 & 92 & 97 \\
\hline $19: 58: \odot \odot$ & 1198 & 101 & 94 & 101 & 94 & 100 & 93 & 100 & 92 & 97 \\
\hline $2 \odot: \odot 3: \odot \odot$ & 1203 & 101 & 94 & 100 & 94 & 100 & 93 & 100 & 92 & 97 \\
\hline 2०:๑৪:๑९ & 1208 & 101 & 94 & 100 & 94 & 100 & 93 & 100 & 92 & 97 \\
\hline $20: 13: \odot \odot$ & 1213 & 101 & 94 & 100 & 94 & 100 & 93 & 100 & 92 & 97 \\
\hline $20: 18: 0 \odot$ & 1218 & 100 & 94 & 100 & 93 & 100 & 93 & 100 & 92 & 97 \\
\hline $20: 23: \odot \odot$ & 1223 & 100 & 94 & 100 & 93 & 100 & 92 & 100 & 92 & 96 \\
\hline $20: 28: \odot \odot$ & 1228 & 100 & 93 & 100 & 93 & 99 & 92 & 100 & 92 & 96 \\
\hline $2 \odot: 33: \odot \odot$ & 1233 & 100 & 93 & 99 & 93 & 99 & 92 & 98 & 91 & 96 \\
\hline $20: 38: \odot \odot$ & 1238 & 100 & 93 & 99 & 93 & 99 & 92 & 98 & 91 & 96 \\
\hline $2 \odot: 43: \odot \odot$ & 1243 & 99 & 93 & 99 & 93 & 99 & 92 & 98 & 91 & 96 \\
\hline $2 \odot: 48: \odot \odot$ & 1248 & 99 & 93 & 99 & 92 & 98 & 92 & 97 & 91 & 95 \\
\hline $2 \odot: 53: \odot \odot$ & 1253 & 99 & 93 & 99 & 92 & 98 & 92 & 97 & 91 & 95 \\
\hline $2 \odot: 58: \odot \odot$ & 1258 & 99 & 92 & 99 & 92 & 98 & 92 & 97 & 91 & 95 \\
\hline 21:०3:๑९ & 1263 & 99 & 92 & 99 & 92 & 98 & 91 & 97 & 90 & 95 \\
\hline 21: ๑৪:๑९ & 1268 & 99 & 92 & 99 & 92 & 98 & 91 & 97 & $9 \odot$ & 95 \\
\hline $21: 13: \odot \odot$ & 1273 & 98 & 92 & 98 & 92 & 98 & 91 & 97 & 90 & 95 \\
\hline $21: 18: 0 \odot$ & 1278 & 98 & 92 & 98 & 92 & 98 & 91 & 97 & $9 \odot$ & 95 \\
\hline $21: 23: \odot \odot$ & 1283 & 98 & 92 & 98 & 92 & 98 & 91 & 97 & $9 \odot$ & 95 \\
\hline $21: 28: \odot \odot$ & 1288 & 98 & 91 & 97 & 91 & 96 & 91 & 96 & 90 & 94 \\
\hline $21: 33: \odot \odot$ & 1293 & 98 & 91 & 97 & 91 & 96 & 91 & 96 & 89 & 94 \\
\hline $21: 38: \odot \odot$ & 1298 & 97 & 91 & 97 & 91 & 96 & 91 & 96 & 89 & 94 \\
\hline $21: 43: \odot \odot$ & 1303 & 97 & 91 & 97 & 91 & 96 & $9 \odot$ & 96 & 89 & 93 \\
\hline $21: 48: \odot \odot$ & 1308 & 97 & 91 & 97 & 91 & 96 & $9 \odot$ & 96 & 89 & 93 \\
\hline $21: 53: \odot \odot$ & 1313 & 97 & 91 & 96 & 91 & 96 & $9 \odot$ & 95 & 89 & 93 \\
\hline $21: 58: \odot \odot$ & 1318 & 97 & 91 & 96 & 91 & 95 & 90 & 95 & 89 & 93 \\
\hline $22: \odot 3: \odot \odot$ & 1323 & 97 & 91 & 96 & 91 & 95 & 90 & 95 & 89 & 93 \\
\hline $22: \odot 8: \odot \odot$ & 1328 & 97 & $9 \odot$ & 96 & 91 & 95 & 90 & 95 & 89 & 93 \\
\hline $22: 13: \odot \odot$ & 1333 & 96 & 90 & 96 & 91 & 95 & $9 \odot$ & 95 & 89 & 93 \\
\hline $22: 18: \odot \odot$ & 1338 & 96 & 90 & 96 & 89 & 95 & 90 & 95 & 89 & 93 \\
\hline $22: 23: \odot \odot$ & 1343 & 96 & 90 & 96 & 89 & 95 & 90 & 95 & 89 & 93 \\
\hline $22: 28: \odot \odot$ & 1348 & 96 & 90 & 96 & 89 & 95 & 90 & 95 & 89 & 93 \\
\hline $22: 33: \odot \odot$ & 1353 & 96 & $9 \odot$ & 96 & 89 & 95 & 90 & 95 & 89 & 93 \\
\hline $22: 38: \odot \odot$ & 1358 & 96 & $9 \odot$ & 96 & 89 & 95 & 90 & 94 & 88 & 92 \\
\hline $22: 43: \odot \odot$ & 1363 & 96 & 90 & 96 & 89 & 95 & 90 & 94 & 88 & 92 \\
\hline $22: 48: \odot \odot$ & 1368 & 96 & $9 \odot$ & 96 & 89 & 94 & $9 \odot$ & 94 & 88 & 92 \\
\hline $22: 53: \odot \odot$ & 1373 & 96 & $9 \odot$ & 96 & 89 & 94 & 90 & 94 & 88 & 92 \\
\hline $22: 58: \odot \odot$ & 1378 & 96 & 90 & 96 & 89 & 94 & $9 \odot$ & 94 & 88 & 92 \\
\hline $23: \odot 3: \odot \odot$ & 1383 & 96 & $9 \odot$ & 96 & 89 & 94 & 89 & 94 & 88 & 92 \\
\hline $23: \odot 8: \odot \odot$ & 1388 & 96 & $9 \odot$ & 96 & 89 & 94 & 89 & 94 & 88 & 92 \\
\hline $23: 13: \odot \odot$ & 1393 & 96 & 90 & 96 & 89 & 94 & 89 & 94 & 88 & 92 \\
\hline $23: 18: \odot \odot$ & 1398 & 96 & $9 \odot$ & 96 & 89 & 94 & 89 & 94 & 88 & 92 \\
\hline $23: 23: \odot \odot$ & 1403 & 96 & 90 & 96 & 89 & 94 & 89 & 94 & 88 & 92 \\
\hline $23: 28: \odot \odot$ & 1408 & 96 & 90 & 96 & 89 & 94 & 89 & 94 & 88 & 92 \\
\hline $23: 33: \odot \odot$ & 1413 & 96 & 90 & 96 & 89 & 94 & 89 & 94 & 88 & 92 \\
\hline $23: 38: \odot \odot$ & 1418 & 96 & 90 & 96 & 89 & 94 & 89 & 94 & 88 & 92 \\
\hline $23: 43: 0 \odot$ & 1423 & 95 & 90 & 96 & 89 & 94 & 89 & 94 & 88 & 92 \\
\hline $23: 48: \odot \odot$ & 1428 & 95 & 90 & 95 & 89 & 94 & 89 & 94 & 88 & 92 \\
\hline $23: 53: \odot \odot$ & 1433 & 95 & 90 & 95 & 89 & 94 & 89 & 94 & 88 & 92 \\
\hline $23: 58: \odot \odot$ & 1438 & 95 & $9 \odot$ & 95 & 89 & 94 & 89 & 94 & 88 & 92 \\
\hline ๑: ०३:०९ & 1443 & 95 & 90 & 95 & 89 & 94 & 89 & 94 & 88 & 92 \\
\hline$\odot: \odot ৪: \odot \odot ~$ & 1448 & 95 & 90 & 95 & 89 & 94 & 89 & 94 & 88 & 92 \\
\hline
\end{tabular}




\begin{tabular}{|c|c|c|c|c|c|c|c|c|c|c|}
\hline$\odot: 13: \odot \odot$ & 1453 & 95 & 90 & 95 & 89 & 94 & 89 & 94 & 88 & 92 \\
\hline$\odot: 18: \odot \odot$ & 1458 & 95 & 90 & 95 & 89 & 94 & 89 & 94 & 88 & 92 \\
\hline$\odot: 23: \odot \odot$ & 1463 & 95 & 90 & 95 & 89 & 94 & 89 & 94 & 88 & 92 \\
\hline$\odot: 28: \odot \odot$ & 1468 & 95 & 90 & 95 & 89 & 94 & 89 & 93 & 88 & 92 \\
\hline$\odot: 33: \odot \odot$ & 1473 & 95 & 90 & 95 & 89 & 94 & 89 & 93 & 88 & 92 \\
\hline$\odot: 38: \odot \odot$ & 1478 & 95 & 90 & 95 & 89 & 94 & 89 & 93 & 88 & 92 \\
\hline$\odot: 43: \odot \odot$ & 1483 & 95 & 90 & 94 & 89 & 93 & 89 & 93 & 88 & 91 \\
\hline$\odot: 48: \odot \odot$ & 1488 & 94 & 90 & 94 & 89 & 93 & 89 & 92 & 88 & 91 \\
\hline$\odot: 53: \odot \odot$ & 1493 & 94 & 89 & 94 & 89 & 93 & 89 & 92 & 87 & 91 \\
\hline$\odot: 58: \odot \odot$ & 1498 & 94 & 89 & 94 & 89 & 93 & 89 & 92 & 87 & 91 \\
\hline 1:๑3:๑९ & 1503 & 94 & 89 & 94 & 89 & 93 & 89 & 92 & 87 & 91 \\
\hline 1:०৪:๑९ & 1508 & 94 & 89 & 94 & 88 & 93 & 88 & 92 & 87 & 91 \\
\hline 1:13:०๑ & 1513 & 94 & 89 & 94 & 88 & 93 & 88 & 92 & 87 & 91 \\
\hline $1: 18: \odot \odot$ & 1518 & 92 & 89 & 93 & 88 & 92 & 88 & 92 & 87 & 90 \\
\hline $1: 23: 0 \odot$ & 1523 & 92 & 89 & 93 & 88 & 92 & 88 & 92 & 87 & 90 \\
\hline $1: 28: \odot \odot$ & 1528 & 92 & 89 & 93 & 88 & 92 & 88 & 92 & 87 & 90 \\
\hline 1: 33:०९ & 1533 & 92 & 89 & 93 & 88 & 92 & 88 & 91 & 87 & 90 \\
\hline $1: 38: \odot \odot$ & 1538 & 92 & 89 & 93 & 88 & 92 & 88 & 91 & 87 & 90 \\
\hline $1: 43: \odot \odot$ & 1543 & 92 & 88 & 92 & 88 & 92 & 88 & 91 & 87 & 90 \\
\hline $1: 48: \odot \odot$ & 1548 & 92 & 88 & 92 & 88 & 92 & 88 & 91 & 87 & 90 \\
\hline $1: 53: \odot \odot$ & 1553 & 92 & 88 & 92 & 88 & 91 & 88 & 91 & 86 & 90 \\
\hline 1:58:०९ & 1558 & 92 & 88 & 92 & 88 & 91 & 88 & 91 & 86 & 90 \\
\hline $2: \odot 3: \odot \odot$ & 1563 & 92 & 88 & 92 & 88 & 91 & 88 & 91 & 86 & 90 \\
\hline 2:๑8:๑९ & 1568 & 92 & 88 & 92 & 88 & 91 & 88 & 91 & 86 & 90 \\
\hline $2: 13: \odot \odot$ & 1573 & 92 & 88 & 92 & 88 & 91 & 88 & 91 & 86 & 90 \\
\hline $2: 18: \odot \odot$ & 1578 & 92 & 88 & 92 & 88 & 91 & 88 & 91 & 86 & 90 \\
\hline $2: 23: \odot \odot$ & 1583 & 92 & 88 & 92 & 88 & 91 & 87 & 91 & 86 & 89 \\
\hline $2: 28: \odot \odot$ & 1588 & 91 & 88 & 92 & 88 & 91 & 87 & 91 & 86 & 89 \\
\hline $2: 33: \odot \odot$ & 1593 & 91 & 88 & 92 & 88 & 91 & 87 & 91 & 86 & 89 \\
\hline $2: 38: \odot \odot$ & 1598 & 91 & 88 & 92 & 88 & 91 & 87 & 91 & 86 & 89 \\
\hline $2: 43: \odot \odot$ & 1603 & 91 & 88 & 92 & 88 & 91 & 87 & 90 & 86 & 89 \\
\hline $2: 48: \odot \odot$ & 1608 & 91 & 88 & 92 & 87 & 91 & 87 & 90 & 86 & 89 \\
\hline $2: 53: \odot \odot$ & 1613 & 91 & 88 & 92 & 87 & 91 & 87 & 90 & 86 & 89 \\
\hline $2: 58: \odot \odot$ & 1618 & 91 & 88 & 92 & 87 & 91 & 87 & 90 & 86 & 89 \\
\hline $3: \odot 3: \odot \odot$ & 1623 & 91 & 88 & 92 & 87 & 91 & 87 & 90 & 86 & 89 \\
\hline 3:०८:๑९ & 1628 & 91 & 87 & 92 & 87 & 91 & 87 & 90 & 86 & 89 \\
\hline $3: 13: \odot \odot$ & 1633 & 91 & 87 & 92 & 87 & 91 & 87 & 90 & 86 & 89 \\
\hline $3: 18: \odot \odot$ & 1638 & 91 & 87 & 91 & 87 & 90 & 87 & 90 & 86 & 89 \\
\hline $3: 23: \odot \odot$ & 1643 & 91 & 87 & 91 & 87 & 90 & 87 & 90 & 86 & 89 \\
\hline $3: 28: \odot \odot$ & 1648 & 91 & 87 & 91 & 87 & 90 & 87 & 90 & 86 & 89 \\
\hline $3: 33: \odot \odot$ & 1653 & 91 & 87 & 91 & 87 & 90 & 87 & 90 & 86 & 89 \\
\hline $3: 38: \odot \odot$ & 1658 & 90 & 87 & 91 & 87 & 90 & 87 & 90 & 85 & 88 \\
\hline $3: 43: \odot \odot$ & 1663 & 90 & 87 & 91 & 86 & 90 & 87 & 90 & 85 & 88 \\
\hline $3: 48: \odot \odot$ & 1668 & $9 \odot$ & 87 & 91 & 86 & 90 & 87 & 89 & 85 & 88 \\
\hline $3: 53: 00$ & 1673 & 90 & 87 & 91 & 86 & 90 & 87 & 89 & 85 & 88 \\
\hline $3: 58: \odot \odot$ & 1678 & 90 & 87 & 91 & 86 & 90 & 86 & 89 & 85 & 88 \\
\hline 4:०3:๑९ & 1683 & 90 & 87 & 89 & 86 & 90 & 86 & 89 & 85 & 88 \\
\hline $4: \odot 8: \odot \odot$ & 1688 & 90 & 86 & 89 & 86 & 89 & 86 & 89 & 85 & 87 \\
\hline $4: 13: \odot \odot$ & 1693 & 90 & 86 & 89 & 86 & 89 & 86 & 89 & 85 & 87 \\
\hline $4: 18: \odot \odot$ & 1698 & 90 & 86 & 89 & 86 & 89 & 86 & 89 & 85 & 87 \\
\hline $4: 23: \odot \odot$ & 1703 & 89 & 86 & 89 & 86 & 89 & 86 & 89 & 85 & 87 \\
\hline $4: 28: \odot \odot$ & 1708 & 89 & 86 & 89 & 86 & 89 & 86 & 89 & 85 & 87 \\
\hline $4: 33: \odot \odot$ & 1713 & 89 & 86 & 89 & 86 & 89 & 86 & 89 & 85 & 87 \\
\hline $4: 38: \odot \odot$ & 1718 & 89 & 86 & 89 & 86 & 89 & 86 & 88 & 85 & 87 \\
\hline $4: 43: \odot \odot$ & 1723 & 89 & 86 & 89 & 86 & 89 & 86 & 88 & 85 & 87 \\
\hline $4: 48: \odot \odot$ & 1728 & 89 & 86 & 88 & 86 & 89 & 86 & 88 & 85 & 87 \\
\hline $4: 53: \odot \odot$ & 1733 & 89 & 86 & 88 & 86 & 88 & 85 & 88 & 85 & 87 \\
\hline
\end{tabular}




\begin{tabular}{|c|c|c|c|c|c|c|c|c|c|c|}
\hline $4: 58: 00$ & 1738 & 89 & 86 & 88 & 86 & 88 & 85 & 88 & 85 & 87 \\
\hline $5: \odot 3: \odot \odot$ & 1743 & 89 & 86 & 88 & 85 & 88 & 85 & 88 & 85 & 87 \\
\hline $5: \odot 8: 0 \odot$ & 1748 & 89 & 86 & 88 & 85 & 88 & 85 & 88 & 84 & 87 \\
\hline $5: 13: 00$ & 1753 & 89 & 86 & 88 & 85 & 88 & 85 & 88 & 84 & 87 \\
\hline $5: 18: \odot \odot$ & 1758 & 88 & 86 & 88 & 85 & 88 & 85 & 88 & 84 & 86 \\
\hline $5: 23: 0 \odot$ & 1763 & 88 & 86 & 88 & 85 & 88 & 85 & 88 & 84 & 86 \\
\hline $5: 28: 0 \odot$ & 1768 & 88 & 85 & 88 & 85 & 88 & 85 & 87 & 84 & 86 \\
\hline $5: 33: \odot \odot$ & 1773 & 88 & 85 & 88 & 85 & 88 & 85 & 87 & 84 & 86 \\
\hline $5: 38: 0 \odot$ & 1778 & 88 & 85 & 88 & 85 & 88 & 85 & 87 & 84 & 86 \\
\hline $5: 43: \odot \odot$ & 1783 & 88 & 85 & 88 & 85 & 88 & 85 & 87 & 84 & 86 \\
\hline $5: 48: \odot \odot$ & 1788 & 88 & 85 & 87 & 85 & 88 & 85 & 87 & 84 & 86 \\
\hline $5: 53: \odot \odot$ & 1793 & 88 & 85 & 87 & 85 & 88 & 85 & 87 & 84 & 86 \\
\hline $5: 58: \odot \odot$ & 1798 & 88 & 85 & 87 & 85 & 88 & 85 & 87 & 84 & 86 \\
\hline $6: 03: 0 \odot$ & 1803 & 88 & 85 & 87 & 85 & 88 & 85 & 87 & 84 & 86 \\
\hline 6:๑৪:๑९ & 1808 & 88 & 85 & 87 & 85 & 88 & 85 & 87 & 84 & 86 \\
\hline $6: 13: 0 \odot$ & 1813 & 88 & 85 & 87 & 85 & 88 & 85 & 87 & 84 & 86 \\
\hline $6: 18: \odot \odot$ & 1818 & 88 & 85 & 87 & 85 & 87 & 85 & 87 & 84 & 86 \\
\hline $6: 23: \odot \odot$ & 1823 & 88 & 85 & 87 & 85 & 87 & 85 & 87 & 84 & 86 \\
\hline $6: 28: 0 \odot$ & 1828 & 88 & 85 & 87 & 85 & 87 & 85 & 87 & 84 & 86 \\
\hline $6: 33: \odot \odot$ & 1833 & 87 & 85 & 87 & 85 & 87 & 85 & 87 & 84 & 86 \\
\hline $6: 38: \odot \odot$ & 1838 & 87 & 85 & 87 & 85 & 87 & 85 & 87 & 84 & 86 \\
\hline $6: 43: 0 \odot$ & 1843 & 87 & 85 & 87 & 85 & 87 & 85 & 87 & 84 & 86 \\
\hline $6: 48: \odot \odot$ & 1848 & 87 & 85 & 87 & 84 & 87 & 85 & 87 & 83 & 85 \\
\hline $6: 53: \odot \odot$ & 1853 & 87 & 85 & 87 & 84 & 87 & 85 & 87 & 83 & 85 \\
\hline $6: 58: \odot \odot$ & 1858 & 87 & 85 & 87 & 84 & 87 & 84 & 87 & 83 & 85 \\
\hline 7:०3:०९ & 1863 & 87 & 85 & 87 & 84 & 87 & 84 & 87 & 83 & 85 \\
\hline 7:๑৪:๑९ & 1868 & 87 & 85 & 87 & 84 & 87 & 84 & 87 & 83 & 85 \\
\hline $7: 13: \odot \odot$ & 1873 & 87 & 85 & 87 & 84 & 87 & 84 & 87 & 83 & 85 \\
\hline $7: 18: \odot \odot$ & 1878 & 87 & 85 & 87 & 84 & 87 & 84 & 87 & 83 & 85 \\
\hline $7: 23: \odot \odot$ & 1883 & 87 & 83 & 86 & 84 & 87 & 84 & 85 & 83 & 85 \\
\hline $7: 28: \odot \odot$ & 1888 & 87 & 83 & 86 & 84 & 87 & 84 & 85 & 83 & 85 \\
\hline $7: 33: \odot \odot$ & 1893 & 87 & 83 & 86 & 84 & 87 & 84 & 85 & 83 & 85 \\
\hline $7: 38: \odot \odot$ & 1898 & 87 & 83 & 86 & 84 & 87 & 84 & 85 & 83 & 85 \\
\hline $7: 43: \odot \odot$ & 1903 & 87 & 83 & 86 & 84 & 87 & 84 & 85 & 83 & 85 \\
\hline $7: 48: \odot \odot$ & 1908 & 87 & 83 & 86 & 84 & 87 & 84 & 85 & 83 & 85 \\
\hline $7: 53: \odot \odot$ & 1913 & 87 & 83 & 86 & 84 & 87 & 84 & 85 & 83 & 85 \\
\hline $7: 58: \odot \odot$ & 1918 & 87 & 83 & 86 & 84 & 87 & 84 & 85 & 83 & 85 \\
\hline ৪:०3:०๑ & 1923 & 87 & 83 & 86 & 84 & 87 & 84 & 85 & 83 & 85 \\
\hline ৪:०৪:०९ & 1928 & 86 & 83 & 86 & 84 & 87 & 84 & 85 & 83 & 85 \\
\hline $8: 13: 0 \odot$ & 1933 & 86 & 83 & 86 & 84 & 87 & 84 & 85 & 83 & 85 \\
\hline 8:18:०๑ & 1938 & 86 & 83 & 86 & 84 & 87 & 84 & 85 & 83 & 85 \\
\hline $8: 23: \odot \odot$ & 1943 & 86 & 83 & 86 & 84 & 86 & 84 & 85 & 83 & 85 \\
\hline ৪:28:๑९ & 1948 & 86 & 83 & 86 & 83 & 86 & 84 & 85 & 82 & 84 \\
\hline 8: $33: \odot \odot$ & 1953 & 86 & 83 & 86 & 83 & 86 & 84 & 85 & 82 & 84 \\
\hline $8: 38: 0 \odot$ & 1958 & 86 & 82 & 86 & 83 & 86 & 82 & 85 & 82 & 84 \\
\hline $8: 43: \odot \odot$ & 1963 & 86 & 82 & 86 & 83 & 86 & 82 & 85 & 82 & 84 \\
\hline ৪:48:๑९ & 1968 & 86 & 82 & 86 & 83 & 86 & 82 & 85 & 82 & 84 \\
\hline $8: 53: \odot \odot$ & 1973 & 86 & 82 & 86 & 83 & 86 & 82 & 85 & 82 & 84 \\
\hline 8:58:๑९ & 1978 & 86 & 82 & 86 & 83 & 86 & 82 & 84 & 82 & 84 \\
\hline 9:०३:०९ & 1983 & 86 & 82 & 86 & 83 & 86 & 82 & 84 & 82 & 84 \\
\hline 9:०८:०० & 1988 & 85 & 82 & 85 & 83 & 86 & 82 & 84 & 82 & 84 \\
\hline $9: 13: \odot \odot$ & 1993 & 85 & 82 & 85 & 83 & 86 & 82 & 84 & 82 & 84 \\
\hline $9: 18: \odot \odot$ & 1998 & 85 & 82 & 85 & 83 & 86 & 82 & 84 & 82 & 84 \\
\hline $9: 23: \odot \odot$ & 2003 & 85 & 82 & 85 & 83 & 86 & 82 & 84 & 82 & 84 \\
\hline $9: 28: \odot \odot$ & 2008 & 85 & 82 & 85 & 83 & 85 & 82 & 84 & 82 & 84 \\
\hline $9: 33: \odot \odot$ & 2013 & 85 & 82 & 85 & 83 & 85 & 82 & 84 & 82 & 84 \\
\hline $9: 38: \odot \odot$ & 2018 & 85 & 82 & 85 & 82 & 85 & 82 & 84 & 82 & 83 \\
\hline
\end{tabular}




\begin{tabular}{|c|c|c|c|c|c|c|c|c|c|c|}
\hline $9: 43: \odot \odot$ & 2023 & 85 & 81 & 84 & 82 & 85 & 82 & 84 & 82 & 83 \\
\hline $9: 48: \odot \odot$ & 2028 & 85 & 81 & 84 & 82 & 85 & 82 & 83 & 82 & 83 \\
\hline $9: 53: 0 \odot$ & 2033 & 85 & 81 & 84 & 82 & 85 & 82 & 83 & 82 & 83 \\
\hline $9: 58: \odot \odot$ & 2038 & 85 & 81 & 84 & 82 & 85 & 82 & 83 & 82 & 83 \\
\hline 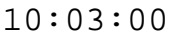 & 2043 & 85 & 81 & 84 & 82 & 85 & 82 & 83 & 81 & 83 \\
\hline 1०: ๑৪:๑९ & 2048 & 85 & 81 & 84 & 82 & 85 & 81 & 83 & 81 & 83 \\
\hline $10: 13: \odot \odot$ & 2053 & 85 & 81 & 84 & 82 & 85 & 81 & 83 & 81 & 83 \\
\hline 1०: $18: \odot \odot$ & 2058 & 85 & 81 & 84 & 82 & 85 & 81 & 83 & 81 & 83 \\
\hline $10: 23: \odot \odot$ & 2063 & 85 & 81 & 84 & 82 & 85 & 81 & 83 & 81 & 83 \\
\hline $10: 28: \odot \odot$ & 2068 & 84 & 81 & 84 & 82 & 83 & 81 & 83 & 81 & 82 \\
\hline $10: 33: \odot \odot$ & 2073 & 84 & 81 & 84 & 82 & 83 & 81 & 83 & 81 & 82 \\
\hline $10: 38: 0 \odot$ & 2078 & 84 & 81 & 84 & 82 & 83 & 81 & 83 & 81 & 82 \\
\hline $10: 43: \odot \odot$ & 2083 & 84 & 81 & 84 & 82 & 83 & 81 & 83 & 81 & 82 \\
\hline $10: 48: \odot \odot$ & 2088 & 84 & 81 & 84 & 82 & 83 & 81 & 83 & 81 & 82 \\
\hline $10: 53: \odot \odot$ & 2093 & 84 & 81 & 84 & 82 & 83 & 81 & 83 & 81 & 82 \\
\hline $10: 58: \odot \odot$ & 2098 & 84 & 81 & 84 & 81 & 83 & 81 & 82 & 81 & 82 \\
\hline $11: \odot 3: \odot \odot$ & 2103 & 84 & 81 & 84 & 81 & 83 & 81 & 82 & 81 & 82 \\
\hline $11: \odot 8: \odot \odot$ & 2108 & 84 & 81 & 83 & 81 & 83 & 81 & 82 & 81 & 82 \\
\hline $11: 13: \odot \odot$ & 2113 & 84 & 81 & 83 & 81 & 83 & 81 & 82 & 81 & 82 \\
\hline $11: 18: 0 \odot$ & 2118 & 84 & 80 & 83 & 81 & 83 & 81 & 82 & 81 & 82 \\
\hline $11: 23: \odot \odot$ & 2123 & 84 & 80 & 83 & 81 & 82 & 81 & 82 & 80 & 82 \\
\hline $11: 28: \odot \odot$ & 2128 & 84 & 80 & 83 & 81 & 82 & 81 & 82 & 80 & 82 \\
\hline $11: 33: \odot \odot$ & 2133 & 83 & 80 & 83 & 81 & 82 & 81 & 82 & 80 & 82 \\
\hline $11: 38: \odot \odot$ & 2138 & 83 & 80 & 83 & 81 & 82 & 81 & 82 & 80 & 82 \\
\hline $11: 43: \odot \odot$ & 2143 & 83 & 80 & 83 & 81 & 82 & 81 & 82 & 80 & 82 \\
\hline $11: 48: \odot \odot$ & 2148 & 83 & 80 & 83 & 81 & 82 & 81 & 82 & 80 & 82 \\
\hline $11: 53: \odot \odot$ & 2153 & 83 & 80 & 83 & 81 & 82 & 81 & 82 & 80 & 82 \\
\hline $11: 58: \odot \odot$ & 2158 & 83 & 80 & 83 & 81 & 82 & 80 & 82 & 80 & 81 \\
\hline $12: \odot 3: \odot \odot$ & 2163 & 83 & 80 & 83 & 81 & 82 & 80 & 82 & 80 & 81 \\
\hline 12: ९৪: :९ & 2168 & 83 & 80 & 83 & 81 & 82 & 80 & 82 & 80 & 81 \\
\hline $12: 13: \odot \odot$ & 2173 & 83 & 80 & 82 & 81 & 82 & 80 & 82 & 80 & 81 \\
\hline $12: 18: \odot \odot$ & 2178 & 83 & 80 & 82 & 81 & 82 & 80 & 82 & 80 & 81 \\
\hline $12: 23: 00$ & 2183 & 83 & 80 & 82 & 81 & 82 & 80 & 81 & 80 & 81 \\
\hline $12: 28: \odot \odot$ & 2188 & 83 & 80 & 82 & 81 & 82 & 80 & 81 & 80 & 81 \\
\hline $12: 33: \odot \odot$ & 2193 & 82 & 80 & 82 & 81 & 82 & 80 & 81 & 80 & 81 \\
\hline $12: 38: 0 \odot$ & 2198 & 82 & 79 & 82 & 80 & 82 & 80 & 81 & 80 & 81 \\
\hline $12: 43: \odot \odot$ & 2203 & 82 & 79 & 82 & 80 & 81 & 80 & 81 & 80 & 81 \\
\hline $12: 48: \odot \odot$ & 2208 & 82 & 79 & 82 & 80 & 81 & 80 & 81 & 78 & 80 \\
\hline $12: 53: \odot \odot$ & 2213 & 82 & 79 & 82 & 80 & 81 & 80 & 81 & 78 & 80 \\
\hline $12: 58: \odot \odot$ & 2218 & 82 & 79 & 82 & 80 & 81 & 80 & 81 & 78 & 80 \\
\hline 13: ๑3:๑९ & 2223 & 82 & 79 & 82 & 80 & 81 & 80 & 81 & 78 & 80 \\
\hline 13: ๑৪:๑९ & 2228 & 82 & 79 & 82 & 80 & 81 & 80 & 81 & 78 & 80 \\
\hline $13: 13: 00$ & 2233 & 82 & 79 & 82 & 80 & 81 & 79 & 81 & 78 & 80 \\
\hline 13: $18: \odot \odot$ & 2238 & 82 & 79 & 82 & 80 & 81 & 79 & 81 & 78 & 80 \\
\hline $13: 23: \odot \odot$ & 2243 & 82 & 79 & 81 & 80 & 81 & 79 & 81 & 78 & 80 \\
\hline $13: 28: \odot \odot$ & 2248 & 82 & 79 & 81 & 80 & 81 & 79 & 80 & 78 & 80 \\
\hline $13: 33: \odot \odot$ & 2253 & 82 & 79 & 81 & 80 & 81 & 79 & 80 & 78 & 80 \\
\hline $13: 38: \odot \odot$ & 2258 & 82 & 79 & 81 & 80 & 81 & 79 & 80 & 78 & 80 \\
\hline $13: 43: \odot \odot$ & 2263 & 82 & 79 & 81 & 80 & 81 & 79 & 80 & 78 & 80 \\
\hline $13: 48: \odot \odot$ & 2268 & 82 & 79 & 81 & 80 & 81 & 79 & 80 & 78 & 80 \\
\hline $13: 53: \odot \odot$ & 2273 & 82 & 79 & 81 & 80 & 81 & 79 & 80 & 78 & 80 \\
\hline $13: 58: \odot \odot$ & 2278 & 82 & 79 & 81 & 80 & 81 & 79 & 80 & 78 & 80 \\
\hline $14: \odot 3: \odot \odot$ & 2283 & 82 & 79 & 81 & 80 & 81 & 79 & 80 & 78 & 80 \\
\hline $14: \odot 8: \odot \odot$ & 2288 & 82 & 79 & 81 & 80 & 81 & 79 & 80 & 78 & 80 \\
\hline $14: 13: \odot \odot$ & 2293 & 82 & 78 & 81 & 80 & 81 & 79 & 80 & 78 & 80 \\
\hline $14: 18: \odot \odot$ & 2298 & 81 & 78 & 81 & 80 & 81 & 79 & 80 & 78 & 80 \\
\hline $14: 23: 0 \odot$ & 2303 & 81 & 78 & 81 & 80 & 81 & 79 & 80 & 78 & 80 \\
\hline
\end{tabular}




\begin{tabular}{|c|c|c|c|c|c|c|c|c|c|}
\hline $14: 28: \odot \odot$ & 2308 & 81 & 78 & 81 & 79 & 81 & 79 & 80 & 78 \\
\hline $14: 33: \odot \odot$ & 2313 & 81 & 78 & 81 & 79 & 81 & 79 & 80 & 78 \\
\hline $14: 38: \odot \odot$ & 2318 & 81 & 78 & 81 & 79 & 81 & 79 & 80 & 78 \\
\hline $14: 43: \odot \odot$ & 2323 & 81 & 78 & 81 & 79 & 80 & 78 & 80 & 78 \\
\hline $14: 48: \odot \odot$ & 2328 & 81 & 78 & 81 & 79 & 80 & 78 & 80 & 78 \\
\hline $14: 53: \odot \odot$ & 2333 & 81 & 78 & 81 & 79 & 80 & 78 & 80 & 78 \\
\hline $14: 58: \odot \odot$ & 2338 & 81 & 78 & 81 & 79 & 80 & 78 & 80 & 78 \\
\hline $15: \odot 3: \odot \odot$ & 2343 & 81 & 78 & 81 & 79 & 80 & 78 & 80 & 78 \\
\hline $15: \odot 8: \odot \odot$ & 2348 & 81 & 78 & 81 & 79 & 80 & 78 & 80 & 78 \\
\hline $15: 13: 0 \odot$ & 2353 & 81 & 78 & 80 & 79 & 80 & 78 & 80 & 78 \\
\hline $15: 18: \odot \odot$ & 2358 & 81 & 78 & 80 & 79 & 80 & 78 & 80 & 77 \\
\hline $15: 23: \odot \odot$ & 2363 & 81 & 78 & 80 & 79 & 80 & 78 & 80 & 77 \\
\hline $15: 28: \odot \odot$ & 2368 & 81 & 78 & 80 & 79 & 80 & 78 & 80 & 77 \\
\hline $15: 33: \odot \odot$ & 2373 & 81 & 78 & 80 & 79 & 80 & 78 & 79 & 77 \\
\hline $15: 38: \odot \odot$ & 2378 & 81 & 78 & 80 & 79 & 80 & 78 & 79 & 77 \\
\hline $15: 43: \odot \odot$ & 2383 & 81 & 78 & 80 & 79 & 80 & 78 & 79 & 77 \\
\hline $15: 48: \odot \odot$ & 2388 & 81 & 78 & 80 & 79 & 80 & 78 & 79 & 77 \\
\hline $15: 53: \odot \odot$ & 2393 & 81 & 78 & 80 & 79 & 80 & 78 & 79 & 77 \\
\hline $15: 58: \odot \odot$ & 2398 & 81 & 78 & 80 & 79 & 80 & 78 & 79 & 77 \\
\hline $16: \odot 3: \odot \odot$ & 2403 & 81 & 78 & 80 & 79 & 80 & 78 & 79 & 77 \\
\hline $16: \odot 8: \odot \odot$ & 2408 & 81 & 78 & 80 & 79 & 80 & 78 & 79 & 77 \\
\hline $16: 13: \odot \odot$ & 2413 & 80 & 78 & 80 & 78 & 80 & 78 & 79 & 77 \\
\hline $16: 18: \odot \odot$ & 2418 & 80 & 78 & 80 & 78 & 80 & 78 & 79 & 77 \\
\hline $16: 23: \odot \odot$ & 2423 & 80 & 78 & 80 & 78 & 79 & 78 & 79 & 77 \\
\hline $16: 28: \odot \odot$ & 2428 & 80 & 78 & 80 & 78 & 79 & 78 & 79 & 77 \\
\hline $16: 33: \odot \odot$ & 2433 & 80 & 78 & 80 & 78 & 79 & 78 & 79 & 77 \\
\hline $16: 38: \odot \odot$ & 2438 & 80 & 78 & 80 & 78 & 79 & 78 & 79 & 77 \\
\hline $16: 43: \odot \odot$ & 2443 & 80 & 78 & 80 & 78 & 79 & 78 & 79 & 77 \\
\hline $16: 48: \odot \odot$ & 2448 & 80 & 77 & $8 \odot$ & 78 & 79 & 78 & 79 & 77 \\
\hline $16: 53: \odot \odot$ & 2453 & 80 & 77 & 80 & 78 & 79 & 78 & 79 & 77 \\
\hline $16: 58: \odot \odot$ & 2458 & 80 & 77 & 80 & 78 & 79 & 78 & 79 & 77 \\
\hline $17: \odot 3: \odot \odot$ & 2463 & 80 & 77 & 80 & 78 & 79 & 77 & 79 & 77 \\
\hline $17: \odot 8: \odot \odot$ & 2468 & 80 & 77 & 80 & 78 & 79 & 77 & 79 & 77 \\
\hline $17: 13: \odot \odot$ & 2473 & 80 & 77 & 80 & 78 & 79 & 77 & 79 & 77 \\
\hline $17: 18: \odot \odot$ & 2478 & 80 & 77 & $8 \odot$ & 78 & 79 & 77 & 78 & 76 \\
\hline $17: 23: \odot \odot$ & 2483 & 80 & 77 & 79 & 78 & 79 & 77 & 78 & 76 \\
\hline $17: 28: \odot \odot$ & 2488 & 80 & 77 & 79 & 78 & 79 & 77 & 78 & 76 \\
\hline $17: 33: \odot \odot$ & 2493 & 80 & 77 & 79 & 78 & 78 & 77 & 78 & 76 \\
\hline $17: 38: \odot \odot$ & 2498 & 80 & 77 & 79 & 78 & 78 & 77 & 78 & 76 \\
\hline
\end{tabular}




\subsection{Steel Thermocouple Temperature Data}

\begin{tabular}{|c|c|c|c|c|c|c|c|c|c|c|c|c|c|c|c|c|c|}
\hline & C \#1 & & & \#4 & & & & & & & & & & & & & \\
\hline in & $\begin{array}{l}\text { Deg. F } \\
71.4\end{array}$ & $\begin{array}{l}\text { Deg. F } \\
70.3\end{array}$ & $\begin{array}{l}\text { Deg. F } \\
71.6\end{array}$ & $\begin{aligned}= & \text { Deg. F } \\
& 68.9\end{aligned}$ & $\begin{array}{l}\text { Deg. F } \\
69.6\end{array}$ & $\begin{array}{l}\text { Deg. F } \\
69.1\end{array}$ & $\begin{array}{l}\text { Deg. F } \\
70.2\end{array}$ & $\begin{array}{l}\text { Deg. F } \\
68.9\end{array}$ & $\begin{array}{l}\text { Deg. } \\
67.6\end{array}$ & $\begin{array}{l}\text { Deg. } \\
69.3\end{array}$ & $\begin{array}{l}\text { Deg. } \\
67.6\end{array}$ & $\begin{array}{l}\text { Deg. } \\
66.4\end{array}$ & $\begin{array}{l}\text { Deg. } \\
66.7\end{array}$ & $\begin{array}{l}\text { Deg. } \\
65.5\end{array}$ & $\begin{array}{l}\text { Deg. } \\
66.7\end{array}$ & $\begin{array}{l}\text { Deg. } \\
70.6\end{array}$ & $\begin{array}{l}\text { Deg. F } \\
68.8\end{array}$ \\
\hline & 85.3 & 80.4 & 85.8 & & .2 & 77.0 & 82.9 & 81.3 & 75.4 & 81.0 & 77.4 & 72.1 & 77.5 & & 3.4 & 81.6 & \\
\hline & 7.5 & 124.2 & 7.5 & 102.4 & 1.8 & 8.6 & 3.3 & 4.2 & 5.9 & 31.9 & 7.6 & 113.2 & 5.2 & & & 5.4 & 2.0 \\
\hline & 0.1 & 165.7 & 5.8 & 131.0 & 7.5 & 0.0 & 3.3 & 1.4 & 8.9 & 9.0 & 5.7 & 52.4 & 74.2 & 3.0 & 27.0 & 63.2 & 60.6 \\
\hline & 6.9 & 94.3 & 4.7 & 54.2 & 0.7 & 7.8 & 4.0 & 7.3 & 5.6 & 01.7 & 1.2 & 185.7 & 12.2 & 8.7 & 6.2 & 00.0 & 96.7 \\
\hline & 9.7 & 35.9 & 11.9 & 77.4 & 6.7 & 29.1 & 4.9 & 3.3 & 5.7 & 29.3 & 2.2 & 13.6 & 38.8 & 7.5 & 33.4 & 28.7 & 25.6 \\
\hline & 2.5 & 74.8 & 78.1 & 208.2 & 5.6 & 65.1 & 89.2 & 0.1 & 63.8 & 66.5 & $\odot .7$ & 46.6 & 83.5 & 3.4 & 13.4 & 65.9 & 62.5 \\
\hline & 7.6 & 97.2 & 309.4 & 235.6 & 2.0 & 298.6 & 326.8 & 6.6 & 97.7 & 97.7 & 3.6 & 77.9 & 19.3 & 8.4 & 2.8 & 97.5 & 94.9 \\
\hline & 5.0 & 35.7 & 34.8 & 260.2 & 1.3 & 327.6 & 5.1 & 8.0 & 326.5 & 22.0 & 0.4 & 4.3 & 49.9 & 1.8 & 1.2 & 23.9 & 22.2 \\
\hline & 9.2 & 366.8 & 366.4 & 290.5 & 1.4 & 357.4 & 392.9 & 0.7 & 356.9 & 56.7 & 1.4 & 32.1 & 77.8 & 5.7 & $\odot .6$ & & 94.4 \\
\hline$\Theta$ & 33.8 & 396.1 & 394.5 & 319.1 & 33.0 & 385.9 & 431.8 & 5.3 & 387.0 & 388.8 & 93.4 & 358.9 & 4.5 & 0.9 & 8.8 & 5.9 & 83.6 \\
\hline 11 & 7.4 & 425.7 & 430.7 & 346.5 & 468.7 & 418.6 & 460.4 & 485.4 & 417.6 & 421.2 & 423.9 & 387.0 & 46.0 & 0.3 & 2.1 & 7.6 & 15.6 \\
\hline 12 & 0.4 & 459.5 & 465.3 & 375.6 & 511.7 & 452.5 & 496.4 & 518.0 & 451.0 & 456.3 & 53.4 & 414.0 & 474.6 & 6.6 & 95.1 & 50.2 & 48.5 \\
\hline 3 & 31.5 & 491.2 & 501.4 & 405.5 & 550.2 & 485.2 & 528.6 & 549.9 & 482.0 & 494.4 & 487.9 & 442.6 & 500.7 & 61.9 & 427.5 & 82.4 & 480.7 \\
\hline 4 & 58.4 & 526.5 & 542.5 & 437.9 & 599.5 & 521.8 & 562.6 & 586.4 & 516.9 & 533.7 & 524.8 & 473.0 & 537.3 & $9 \odot . \odot$ & 462.4 & 518.8 & 517.1 \\
\hline 15 & 06.4 & 561.0 & 582.1 & 468.5 & 640.2 & 557.1 & 594.5 & 623.7 & 550.6 & 570.7 & 560.8 & 503.2 & 565.7 & 19.0 & 497.5 & 54.5 & 51.8 \\
\hline 16 & 45.3 & 596.3 & 627.1 & 502.5 & 693.9 & 596.1 & 625.1 & 660.9 & 583.9 & 625.6 & 601.5 & 534.0 & 92.9 & 445.8 & 536.2 & 92.8 & 89.5 \\
\hline 17 & 37.7 & 638.4 & 680.5 & 541.2 & 742.1 & 636.6 & 669.4 & 710.8 & 630.3 & 678.0 & 643.1 & 574.2 & 680.5 & 486.1 & 573.4 & 37.0 & 35.5 \\
\hline 18 & 25.9 & 678.9 & 732.4 & 578.1 & 790.5 & 678.9 & 716.0 & 761.0 & 677.5 & 729.1 & 682.5 & 618.3 & 772.5 & 528.1 & 611.1 & 678.8 & 681.5 \\
\hline 19 & $7 \odot .4$ & 722.1 & 781.2 & 614.3 & 839.7 & 721.6 & 765.0 & 811.8 & 725.9 & 783.0 & 722.1 & 663.8 & 849.9 & 572.5 & 8.7 & 722.0 & 727.8 \\
\hline 20 & 27.4 & 774.0 & 830.8 & 652.8 & 887.9 & 772.5 & 837.9 & 872.8 & 783.7 & 838.0 & 765.5 & 714.6 & 9.7 & 621.5 & 688.8 & 771.3 & 780.8 \\
\hline 21 & 69.4 & 817.5 & 879.1 & 686.1 & 932.9 & 821.5 & 885.9 & 924.1 & 833.7 & 889.2 & 806.7 & 762.3 & 1006.2 & 663.1 & 726.4 & 813.0 & 826.3 \\
\hline 22 & 94.2 & 853.2 & 923.2 & 717.6 & 977.7 & 867.4 & 907.0 & 950.4 & 870.6 & 939.6 & 851.0 & 808.5 & 1022.0 & 701.2 & 766.0 & 847.0 & 862.8 \\
\hline 23 & 27.1 & 889.0 & 966.0 & 749.8 & 1022.7 & 903.6 & 934.5 & 986.5 & 908.6 & 982.9 & 896.2 & 844.5 & 1046.8 & 735.3 & 803.1 & 883.0 & 9.4 \\
\hline 24 & 64.8 & 931.5 & 1010.3 & 3787.5 & 1072.8 & 946.4 & 966.9 & 1024.3 & 950.4 & 1031.4 & 947.5 & 7.5 & 1074.6 & 772.7 & 846.0 & 923.5 & 940.8 \\
\hline 25 & ๑७ . 8 & 974.5 & 1045.8 & 3824.9 & 1108.4 & 986.9 & 1014.8 & 1082.7 & 1001.7 & 1068.6 & 991.8 & 1.6 & 1138.6 & 817.5 & 887.4 & 963.2 & 984.7 \\
\hline 26 & 046.1 & 1012.1 & 1077.6 & 862.2 & 1142.2 & 1027.2 & 1054.6 & 1131.3 & 1046.5 & 1105.3 & 1036.9 & 979.3 & 1186.9 & 860.7 & 929.7 & 999.5 & 1025.4 \\
\hline 7 & 081.0 & 1045.9 & 1104.1 & L 898.7 & 1170.7 & 1063.9 & 1086.8 & 1167.3 & 1084.5 & 1135.9 & 1083.0 & 1025.8 & 1210.8 & 4.8 & 972.0 & 1032.4 & 1061.2 \\
\hline 8 & 125.3 & 1083.7 & 1125.5 & 934.5 & 1197.3 & 1098.3 & 1127.3 & 1206.1 & 1121.9 & 1164.6 & 19.9 & 1066.6 & 1238.0 & 5.9 & 1010.3 & 1067. & 1096.5 \\
\hline 9 & 4.1 & 1123.0 & 1148.0 & 969.6 & 1218.7 & 1131.1 & 1175.0 & 1241.8 & 11 & 1187.6 & 50.0 & 1100.5 & 258.0 & 982.6 & 1044.5 & $\odot 3.7$ & 1129.8 \\
\hline$\odot$ & 209.7 & 1156.3 & 1170.9 & 1006.5 & 1243.2 & 1165.8 & 1212.3 & 1278.3 & 1 & 1213.5 & 84.0 & 1136.8 & 1287.3 & $\odot 2 \odot . \odot$ & 1080. & 35.9 & 1162.7 \\
\hline 1 & 245.0 & 1190.3 & 1195.3 & 31041.6 & 1267.7 & 99.7 & 1250.6 & 1311.3 & 12 & 1239.3 & 14.8 & 1171.6 & 315.6 & 56 & 14 & 68 & 194.6 \\
\hline 2 & 267.2 & 1215 & 1219.1 & L 10 & 1288.0 & 1229.5 & 1278.0 & 4.3 & 12 & 3.2 & 243.2 & 1204.0 & & & & 4. & 1221.8 \\
\hline 3 & & 124 & 1243.4 & 8.0 & 131 & & & 13 & & 12 & & & & & & 122 & 1249.4 \\
\hline 4 & & & 1267 & & & & & & & & & & & & & & 1273.1 \\
\hline 5 & & 127 & 1289.5 & & & & & & & & & & & & & & 1292.9 \\
\hline 6 & 1328.0 & 1296.7 & 309.6 & & & & & & & & & & & & & & 14.3 \\
\hline 37 & 1338.8 & & $32 \varepsilon$ & 1206.3 & & & & & & & & & & & & & 1332.5 \\
\hline 38 & 3.2 & & 344 & 1229.2 & 1400 & & & & & & & & & & & & 1352.5 \\
\hline 39 & & & & 1249.3 & & & & & & & & & & & & & 369.5 \\
\hline C & 1385.1 & 1359.5 & $13 / 2.0$ & $1 \angle 09 . \perp$ & & I. & كי . & 1410.0 & $14+2.1$ & $14<0.0$ & $14+0$. & (3) & (4) & ז I & טצט. & 1040.0 & 1387.1 \\
\hline
\end{tabular}


1402.01375 .51387 .01288 .41445 .01400 .41429 .71492 .51431 .71440 .11435 .61399 .51512 .51322 .81347 .61363 .21403 .2 1419.31391 .01401 .61306 .91460 .8 1419.4 1450.4 1511.4 1453.3 1457.1 1454.51419 .41532 .31340 .11363 .81379 .71421 .5 1435.6 1407.6 1416.7 1323.7 1476.5 1439.4 1469.1 1527.3 1472.4 1473.6 1472.7 1441.2 1547.1 1357.0 1380.0 1395.9 1438.6 1453.3 1427.0 1433.5 1340.11492 .71459 .4 1490.2 1546.01493 .41489 .31492 .71461 .41566 .01374 .61396 .61413 .51457 .7 1466.61444 .11448 .61354 .81504 .61478 .11506 .41558 .21512 .01504 .91508 .41481 .21583 .11392 .11415 .11428 .51474 .0 1477.81459 .21462 .11367 .11517 .41494 .01520 .41568 .51525 .31519 .51520 .11499 .01593 .01408 .81434 .21441 .51488 .0 1489.31474 .01473 .61379 .11525 .61508 .41533 .61577 .31538 .61530 .51531 .81512 .11604 .11427 .41451 .11454 .01501 .0 1500.4 1488.2 1485.5 1392.1 1536.8 1521.0 1546.9 1587.4 1552.3 1541.1 1542.7 1525.3 1619.2 1446.8 1467.11466 .71514 .5 1512.51502 .81498 .51405 .61549 .21535 .71559 .81601 .41565 .11553 .91554 .81540 .91632 .71466 .21483 .91479 .81529 .4 1522.21514 .71510 .01420 .31557 .01547 .11570 .61609 .71574 .81564 .31564 .91552 .61644 .61483 .21499 .21491 .81540 .8 1532.51526 .41519 .51435 .11566 .01557 .51581 .61621 .41585 .81573 .91574 .61564 .21655 .21499 .21512 .51503 .41552 .3 1541.51537 .31531 .61449 .91575 .11568 .31593 .11631 .51597 .51583 .21584 .91575 .01670 .51514 .81525 .31514 .31564 .6 1551.21548 .01541 .71464 .81584 .71578 .4 1605.6 1644.6 1612.8 1594.4 1595.1 1588.6 1681.9 1530.71537 .51526 .91576 .6 1562.01559 .71550 .51479 .41594 .41590 .31617 .31655 .81627 .01607 .01607 .41603 .61694 .71545 .11549 .21538 .01589 .3 1569.2 1568.7 1559.3 1493.4 1604.5 1602.9 1628.6 1662.8 1638.1 1616.5 1617.8 1617.8 1701.1 1558.0 1558.2 1548.1 1599.5 1576.0 1575.7 1568.5 1505. 31612.81613 .71634 .51669 .11647 .11626 .61626 .81630 .21704 .91569 .41569 .41555 .51607 .6 1579.11580 .01570 .61512 .11615 .31621 .01636 .91668 .71650 .71631 .51629 .51635 .61706 .01579 .11576 .01560 .51612 .1 1578.91582 .51570 .81518 .81615 .81623 .41637 .11666 .61651 .81631 .11630 .61638 .71698 .31587 .91584 .31563 .11613 .2 1581.31586 .51574 .41526 .41618 .01625 .91637 .11665 .51652 .91634 .91633 .81641 .91700 .81597 .81593 .11566 .91617 .2 1587.91593 .51582 .01536 .61626 .31630 .01645 .31672 .51657 .01641 .61639 .61646 .41706 .51610 .21604 .11575 .21624 .1 1601.81603 .61588 .81545 .61637 .11638 .71656 .11684 .81666 .01652 .01648 .21653 .41715 .01621 .01614 .71585 .01633 .2 1607.51609 .51597 .51553 .71641 .91646 .41661 .91690 .01671 .81659 .01655 .41658 .81719 .01631 .11625 .71592 .41639 .9 1617.4 1620.5 1604.8 1560.4 1651.6 1652.9 1672.7 1698.1 1678.3 1665.0 1661.2 1665.7 1723.6 1643.4 1634.0 1600.1 1647.6 1624.81626 .41613 .31568 .11659 .61660 .81677 .21703 .31684 .91674 .11668 .61673 .61730 .31652 .41644 .41608 .21654 .9 1634.41636 .31623 .61576 .81668 .91669 .51685 .51711 .41692 .71682 .61675 .61680 .41737 .71664 .11654 .91617 .81663 .9 1641.91643 .01631 .51585 .21678 .31676 .81690 .21717 .71699 .01691 .41683 .11689 .41745 .11674 .51664 .21625 .81670 .8 1649.8 1651.8 1640.7 1594.9 1687.1 1684.6 1699.๑ 1724.0 1705.6 1700.4 1690.2 1696.5 1748.7 1683.5 1672.7 1634.5 1678.9 1658.11658 .71648 .21605 .21696 .61691 .11703 .81730 .11713 .01708 .31696 .51703 .11756 .41693 .01680 .41642 .21686 .8 1660.81661 .91652 .91612 .81699 .71695 .21705 .31730 .11714 .51710 .71699 .71707 .11756 .41699 .21686 .41647 .11689 .8 1663.5 1666.0 1654.2 1620.1 1702.6 1696.5 1707.1 1731.0 1717.๑ 1711.6 1701.1 1708.5 1755.7 1705.3 1692.3 1651.1 1692.5 1666.2 1668.4 1657.9 1629.0 1706.7 1700.2 1709.2 1731.0 1718.4 1715.9 1704.4 1712.1 1758.9 1709.6 1696.1 1655.7 1696.2 1671.31672 .51662 .11636 .31708 .71702 .61712 .31735 .21722 .91718 .11707 .41714 .51763 .61714 .51700 .41659 .61699 .3 1677.01677 .21667 .51642 .61713 .61706 .21718 .41739 .81726 .01719 .91709 .21717 .31767 .71719 .71704 .91666 .31704 .0 1681.2 1681.0 1669.8 1648.2 1714.6 1709.8 1723.3 1743.4 1729.2 1722.7 1711.81720 .81773 .31723 .81708 .71670 .41707 .7 1686.4 1685.1 1673.6 1654.2 1719.0 1713.7 1727.11746 .71731 .71725 .61713 .21723 .51774 .01726 .71712 .51675 .11710 .8 1627.71642 .51620 .31628 .81653 .61682 .21656 .11673 .11684 .21655 .21652 .41682 .41686 .41703 .71685 .81629 .81659 .9 1564.91585 .91560 .61594 .61584 .71620 .31585 .01601 .11618 .51587 .21584 .01615 .81604 .71669 .51653 .41576 .51600 .1 1513.2 1534.3 1510.3 1563.3 1531.9 1563.4 1529.8 1541.1 1560.0 1530.9 1528.9 1556.1 1540.0 1630.8 1617.4 1530.3 1549.0 1468.6 1486.6 1467.0 1531.9 1485.7 1511.2 1480.5 1490.0 1506.2 1480.5 1479.9 1501.5 1483.5 1590.6 1579.3 1488.5 1502.5 1445.41453 .51445 .51503 .51451 .71469 .31447 .91453 .11463 .01446 .31447 .71459 .61445 .71555 .01545 .81462 .01469 .1 1422.91439 .11423 .61474 .51430 .41452 .01425 .41430 .11442 .31423 .91425 .21441 .81421 .11517 .41509 .81440 .01445 .6 1401.31418 .51402 .21456 .91409 .71433 .51402 .31407 .61423 .81401 .61402 .71421 .21396 .81483 .31477 .61419 .71423 .3 1380.2 1395.7 1382.0 1442.8 1388.8 1410.1 1380.6 1385.4 1400.5 1380.0 1382.0 1397.1 1374.1 1460.11455 .11400 .21402 .1 1360.61374 .61362 .61429 .51370 .71387 .81361 .11364 .91378 .21359 .11363 .11374 .81354 .31453 .61446 .41381 .81383 .9 1342.2 1356.3 1344.6 1415.7 1352.7 1368.3 1341.9 1345.3 1358.8 1341.0 1344.2 1354.8 1333.91440 .71435 .31364 .71366 .1 1323.51337 .01326 .41400 .91334 .81348 .51322 .21326 .01338 .61321 .91325 .31333 .81313 .81424 .71419 .81346 .91347 .7 1304.81317 .41307 .51385 .81317 .61327 .81303 .31306 .41317 .61301 .21306 .61312 .01293 .31406 .31402 .31328 .91328 .6 
1286.6 1297.8 1290.2 1371.4 1300.5 1307.5 1284.8 1287.3 1297.01283.4 1288.2 1291.11274.01388.8 1385.61311 .51310 .4 1269.11278 .71272 .21357 .01283 .21287 .51266 .41268 .81276 .91265 .21270 .01269 .91254 .61373 .41370 .11294 .31292 .4 1251.01259 .11254 .41342 .61265 .71267 .21247 .71250 .41256 .41246 .81251 .51249 .21234 .81357 .31355 .01276 .71274 .3 1235.11242 .01238 .71329 .41250 .21249 .01231 .71233 .71238 .41231 .01234 .21229 .91217 .71341 .91340 .41261 .31258 .3 1217.71223 .11221 .41314 .51233 .91229 .71213 .91215 .71218 .61212 .11216 .91209 .91199 .11324 .41324 .21244 .21240 .4 1202.71206 .91206 .71301 .01219 .11212 .61198 .41199 .71201 .61196 .81201 .11192 .11183 .61308 .41308 .91229 .31224 .2 1186.3 1189.0 1189.9 1285.2 1202.4 1194.1 1180.9 1182.7 1182.4 1180.4 1183.5 1173.0 1165.3 1290.4 1292.0 1212.6 1207.1 1170.31172 .11174 .31270 .01187 .11176 .81165 .11166 .41165 .51163 .81167 .11155 .21148 .91272 .71275 .31196 .71191 .3 1155.21155 .91158 .81254 .41172 .51160 .21150 .01150 .91149 .11148 .91151 .61139 .51134 .51254 .91258 .31181 .11175 .7 1140.81141 .31144 .91238 .21156 .31145 .11135 .61136 .51134 .51135 .41136 .51125 .01119 .91236 .61241 .21166 .31160 .6 1127.81128 .91132 .51223 .41143 .11132 .51123 .31122 .31121 .21122 .81122 .41110 .41106 .21220 .21225 .21153 .21146 .7 1114.51114 .91119 .01207 .21129 .61117 .61108 .41107 .71105 .51109 .31107 .71094 .21090 .81202 .01207 .91138 .91132 .1 1101.21100 .51106 .21191 .41116 .71102 .61094 .51092 .71089 .91096 .01093 .81078 .51076 .21184 .51191 .41124 .61117 .6 1087.71086 .1 1092.0 1176.1 1103.4 1087.9 1081.0 1078.5 1075.3 1081.9 1080.0 1063.2 1062.1 1167.81175 .21110 .51103 .4 1074.2 1071.9 1078.3 1160.2 1089.9 1072.9 1067.0 1064.3 1060.2 1067.7 1065.6 1048.3 1047.2 1151.6 1159.0 1096.2 1088.8 1061. 21058.41065 .61146 .61076 .71059 .11053 .91049 .71046 .11054 .91051 .21033 .51033 .51137 .91144 .41082 .91075 .1 1049.21045 .91053 .11134 .91065 .01045 .91041 .31037 .51033 .31042 .01040 .01020 .91020 .71125 .01132 .31070 .81063 .1 1036. 2 1032. 4 1040.2 1122. 1 1051.9 1032. 3 1027.9 1024.0 1019.7 1028.7 1025.1 1006.7 1006.5 1109.1 1118.5 1057.7 1049.4 1023.6 1019.8 1028.3 1108.9 1039.1 1019.3 1015.5 1010.8 1006.3 1016.2 1012.5 993.6 994.3 1093.5 1104.3 1045.2 1036.4 1011.71007 .21016 .1 1095.3 1027.6 1006.7 1003.3 998.8 993.7 $1003.11000 .0980 .8 \quad 982.2 \quad 1078.01089 .51032 .61023 .7$

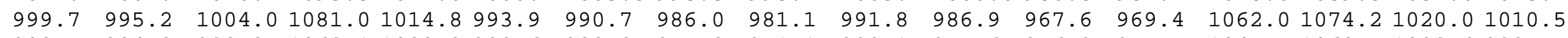

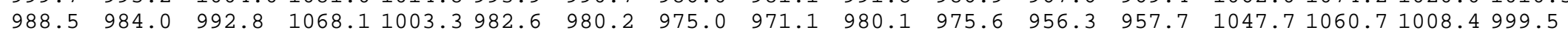

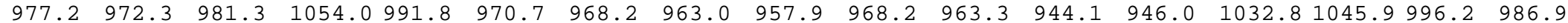

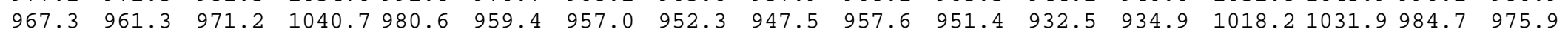

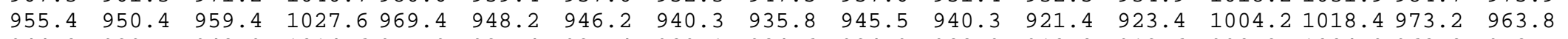

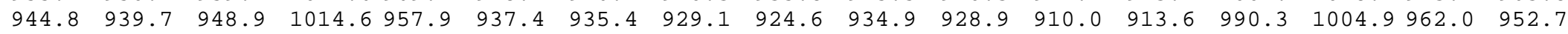

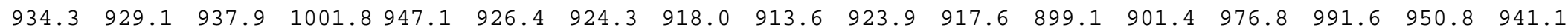

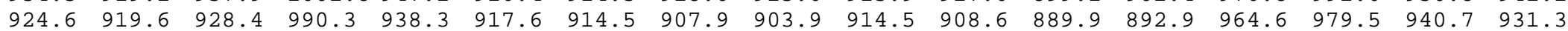

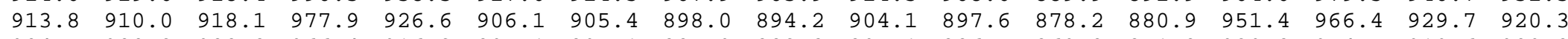

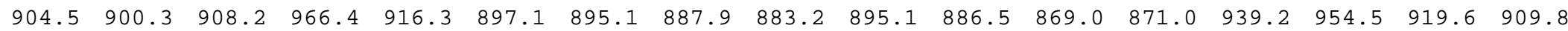

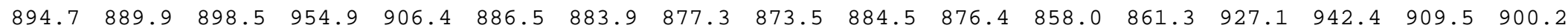

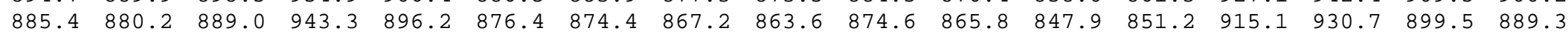
$\begin{array}{lllllllllllllllll}876.0 & 871.0 & 879.6 & 932.4 & 886.5 & 867.0 & 864.7 & 857.8 & 853.9 & 865.6 & 856.2 & 838.0 & 842.2 & 903.6 & 919.2 & 889.7 & 879.0\end{array}$

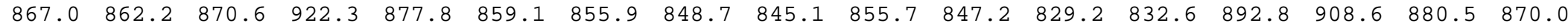

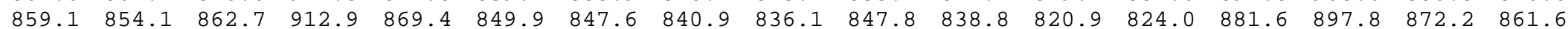

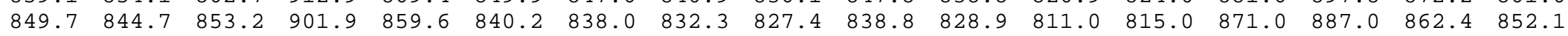

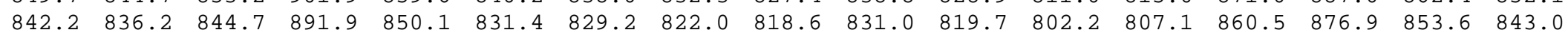

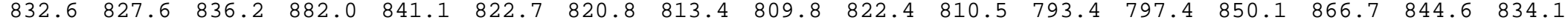

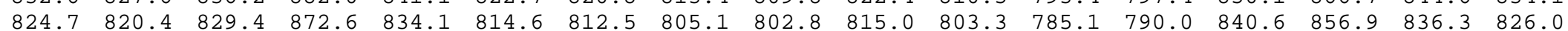

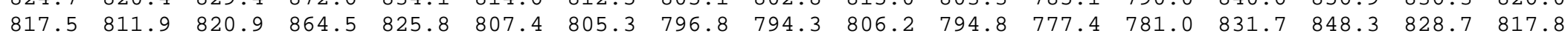

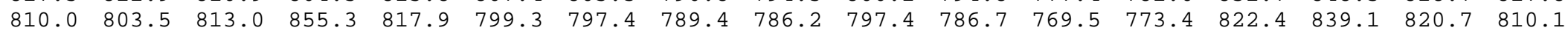

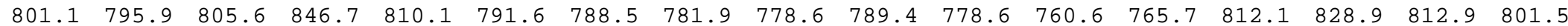

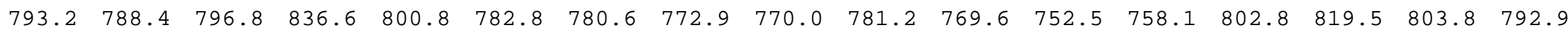

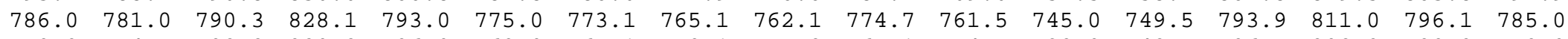

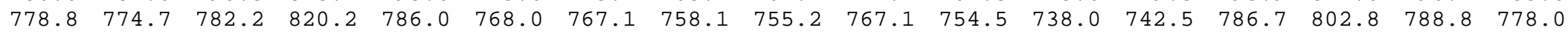

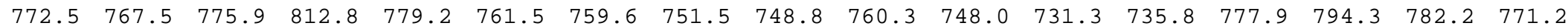

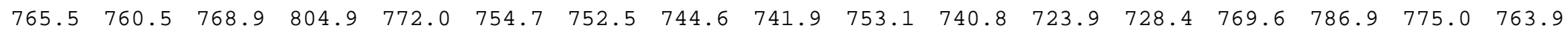




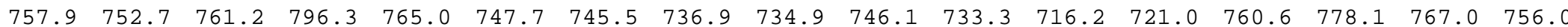

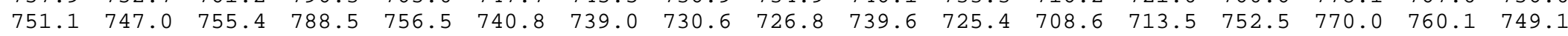

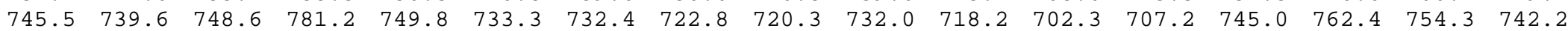

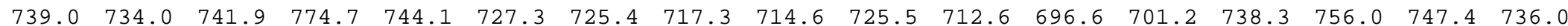

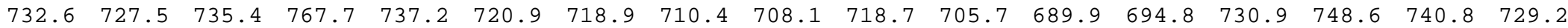

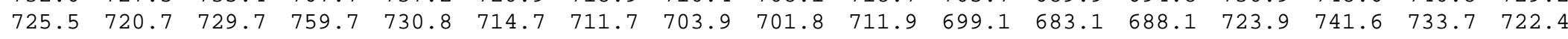

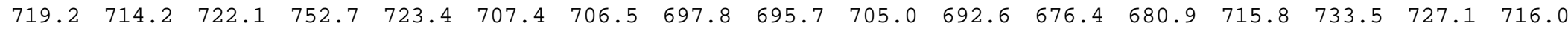

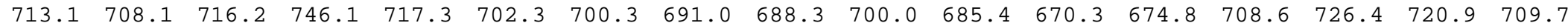

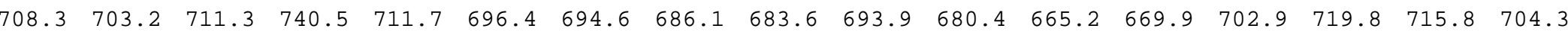

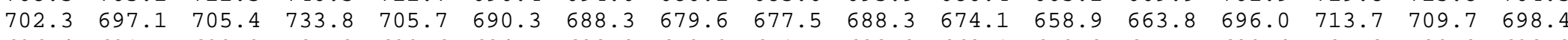

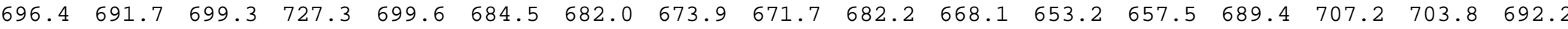

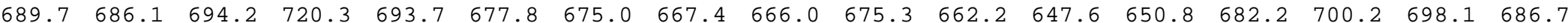

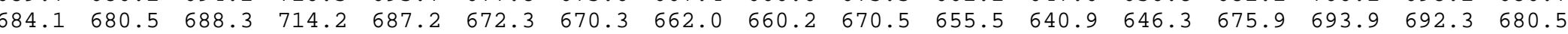

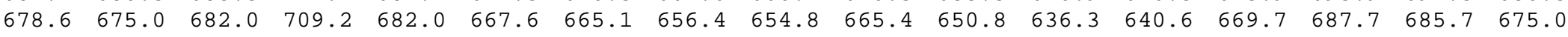

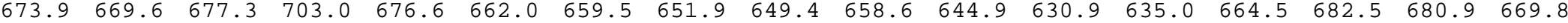

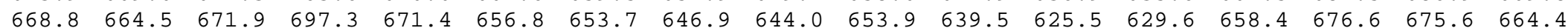

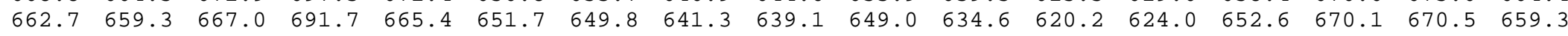

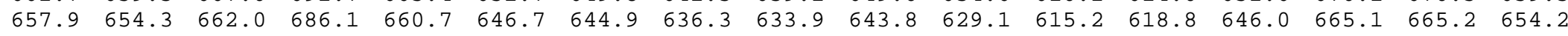

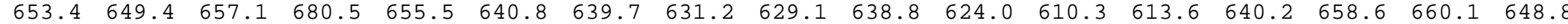

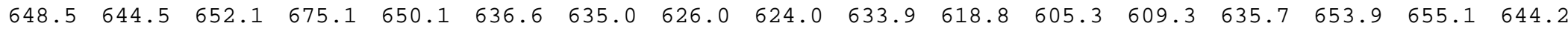

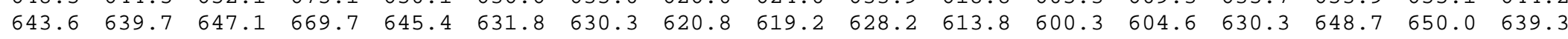

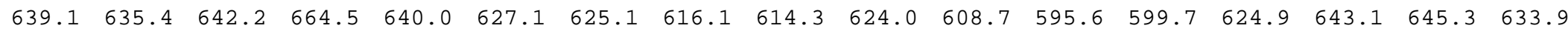

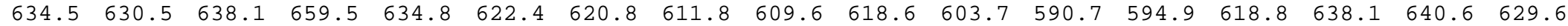

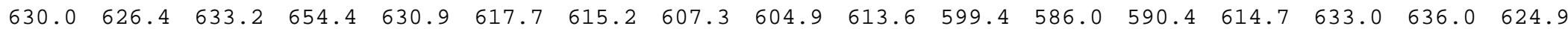

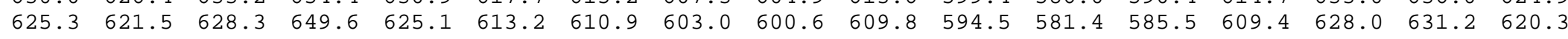

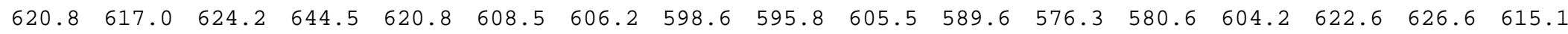

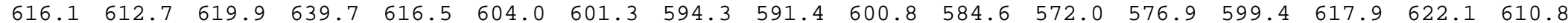

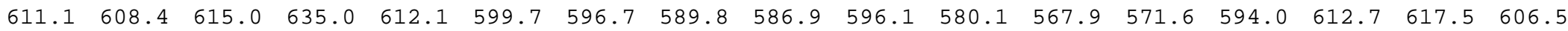

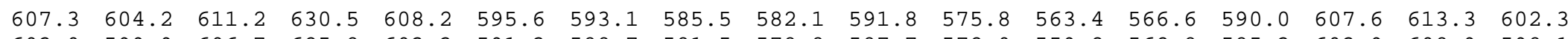

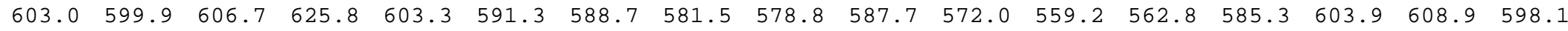

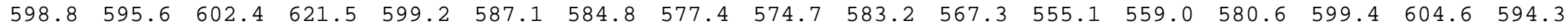

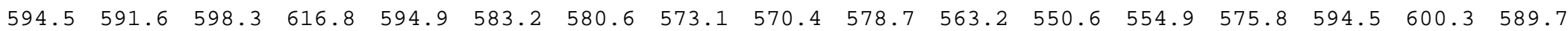

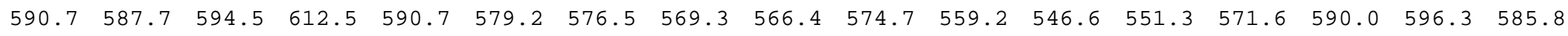

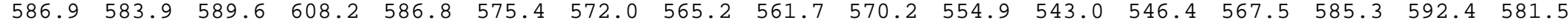

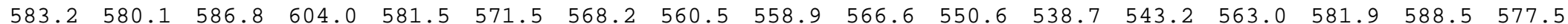
$\begin{array}{lllllllllllllllll}578.8 & 576.3 & 582.6 & 599.7 & 578.1 & 567.5 & 565.0 & 557.4 & 554.7 & 563.4 & 546.8 & 534.9 & 539.1 & 558.9 & 577.4 & 584.6 & 573.7\end{array}$

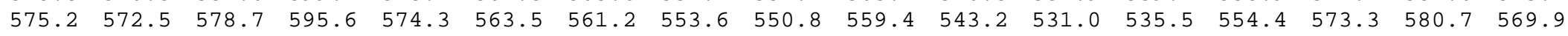

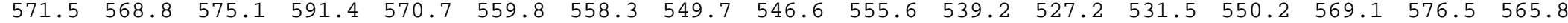

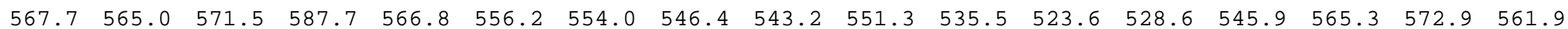

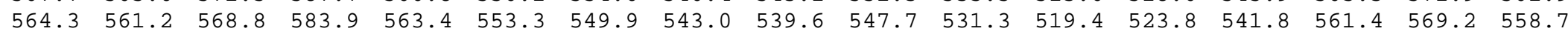

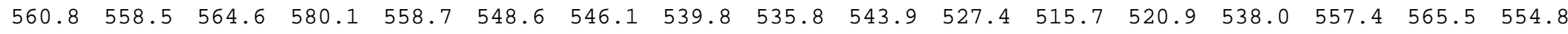

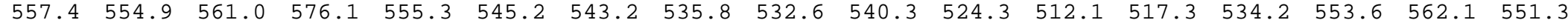

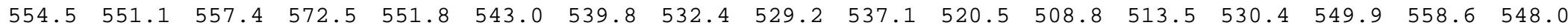

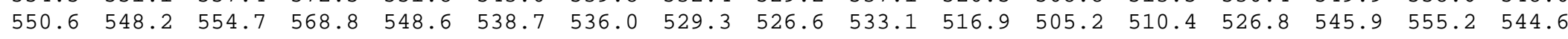

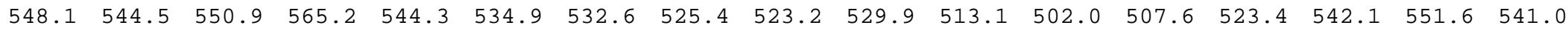

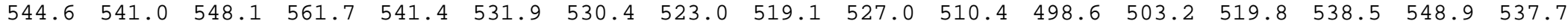
$\begin{array}{llllllllllllllllll}540.9 & 538.0 & 544.3 & 557.8 & 537.8 & 528.6 & 526.1 & 519.1 & 516.0 & 523.2 & 506.8 & 495.3 & 500.0 & 515.7 & 534.9 & 545.5 & 534.3\end{array}$ 
$\begin{array}{llllllllllllllllll}537.4 & 535.8 & 541.0 & 554.4 & 534.6 & 525.7 & 523.2 & 515.8 & 512.4 & 519.6 & 503.4 & 492.4 & 497.7 & 512.4 & 531.9 & 542.0 & 531.2\end{array}$

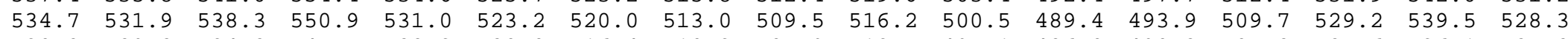

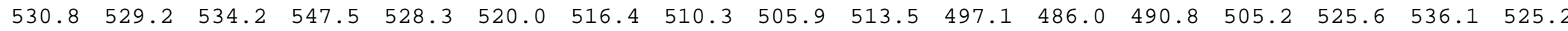

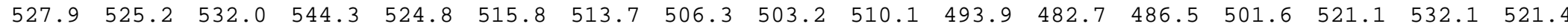

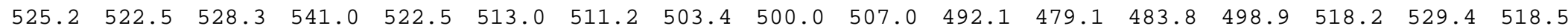

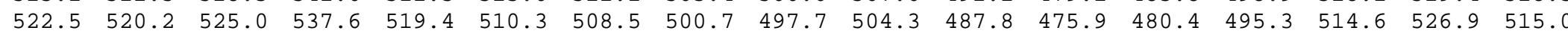

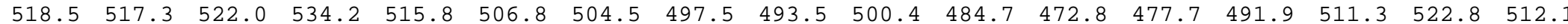

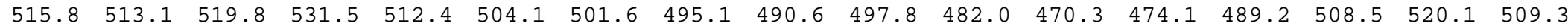

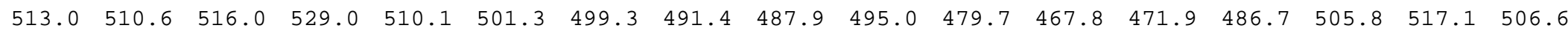

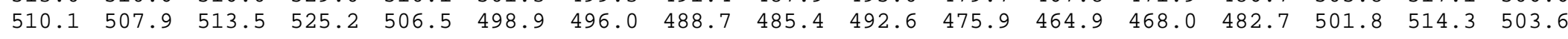

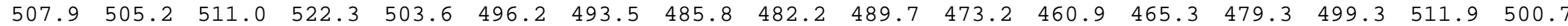

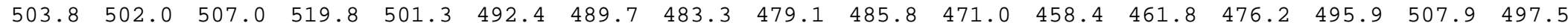

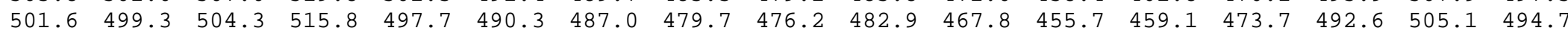

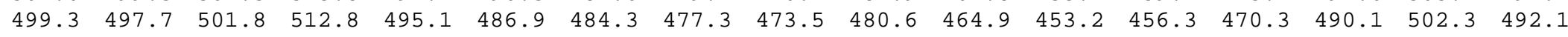

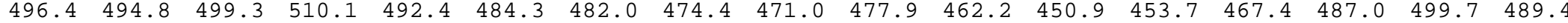

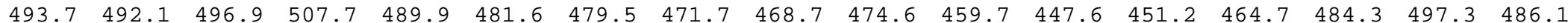

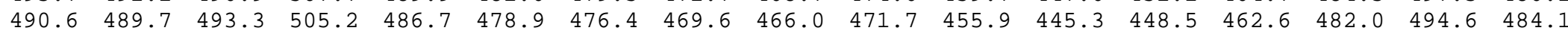

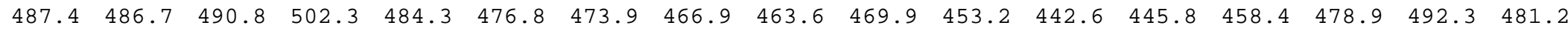

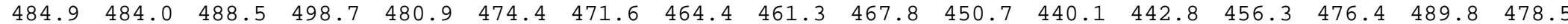

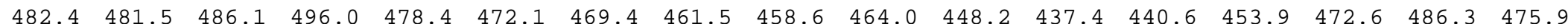

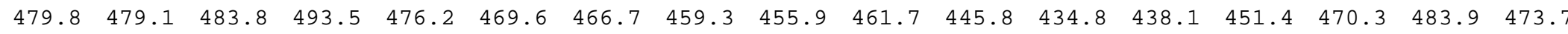

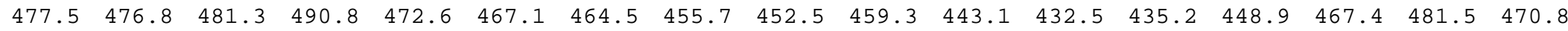

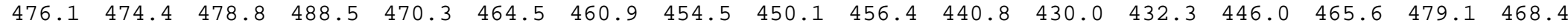

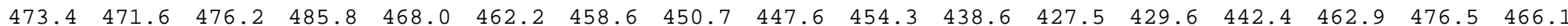

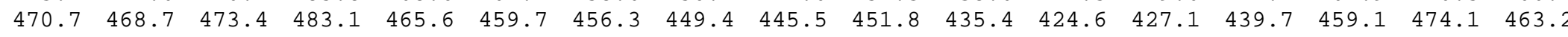

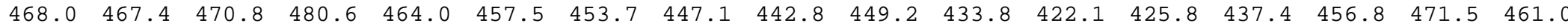

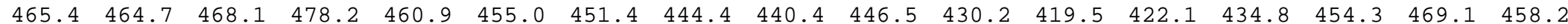

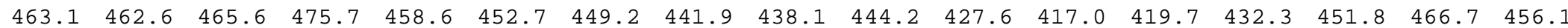

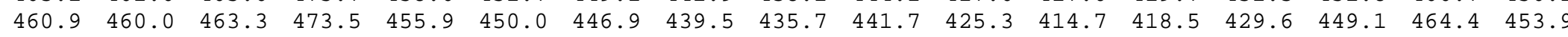

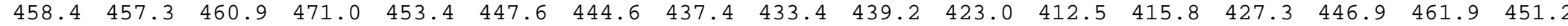

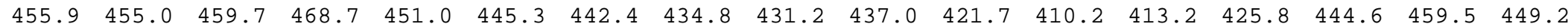

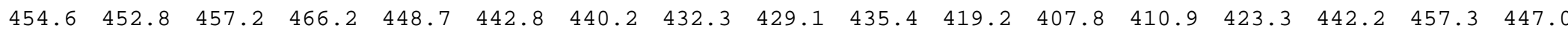

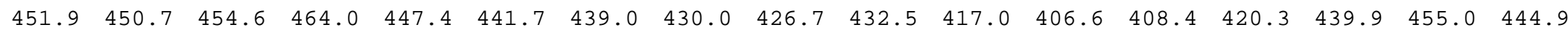

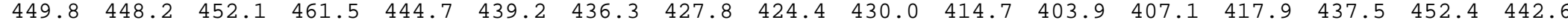

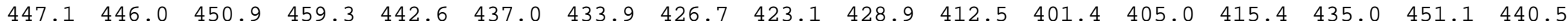

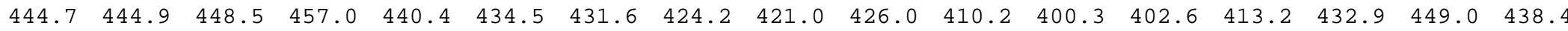

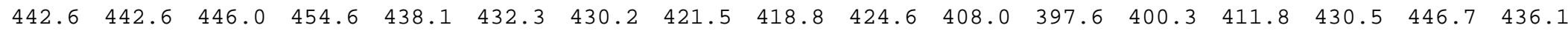

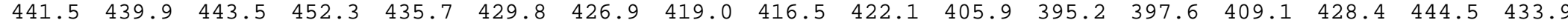

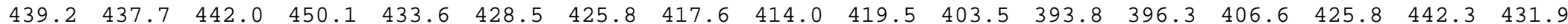

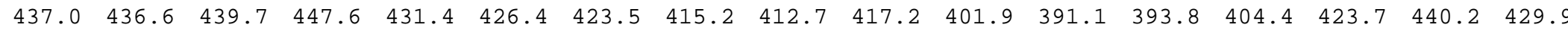

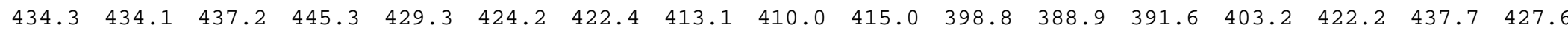

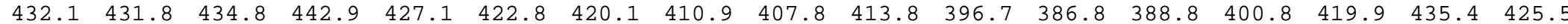

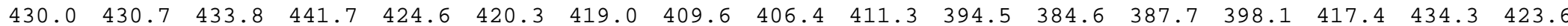

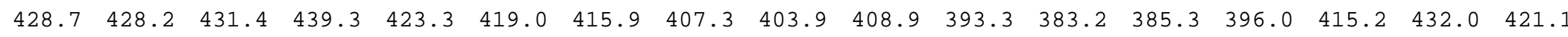

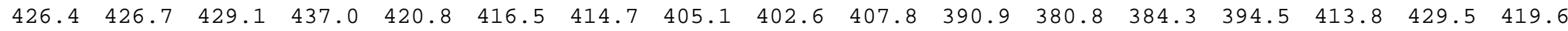

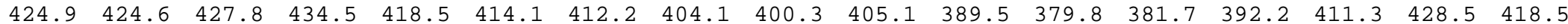

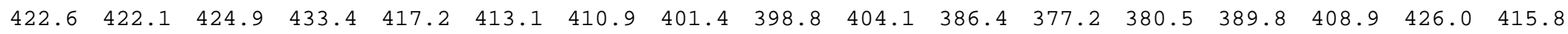




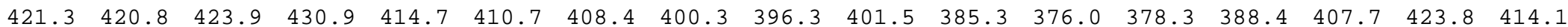

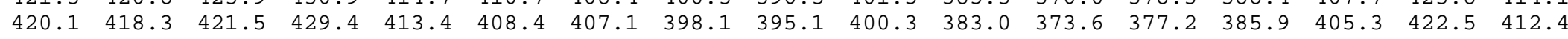

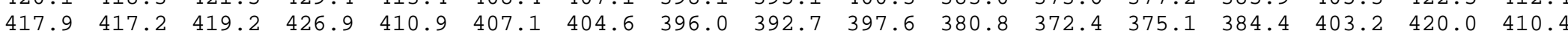

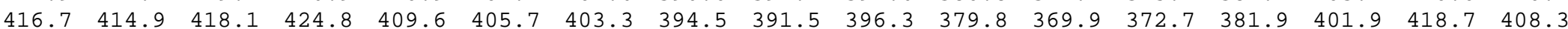

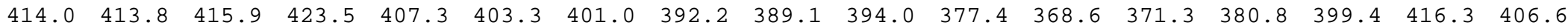

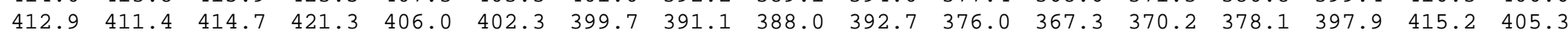

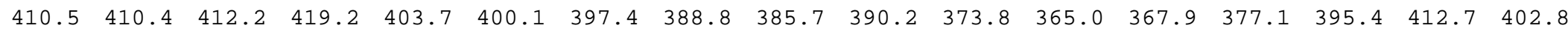

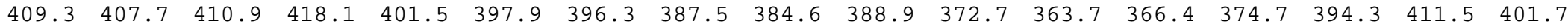

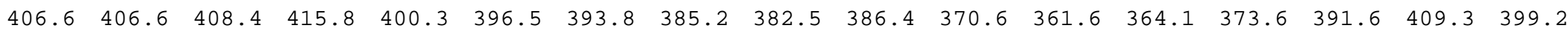

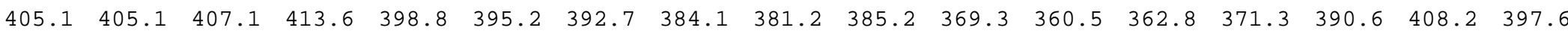

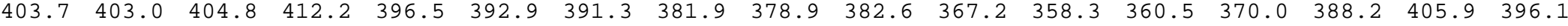

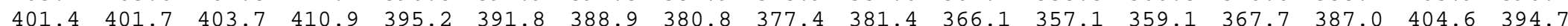

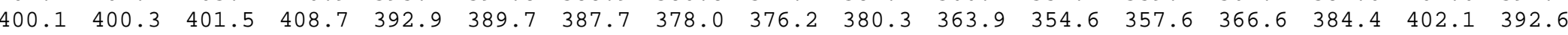

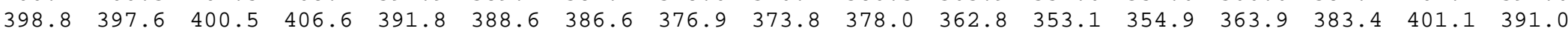
$\begin{array}{lllllllllllllllll}396.5 & 396.5 & 398.3 & 405.5 & 389.5 & 386.2 & 384.4 & 375.4 & 372.7 & 376.7 & 360.1 & 352.0 & 353.8 & 362.7 & 380.8 & 399.9 & 389.4\end{array}$

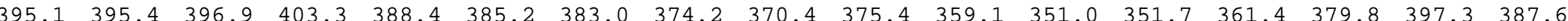

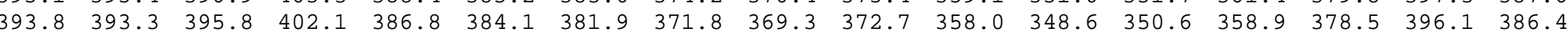

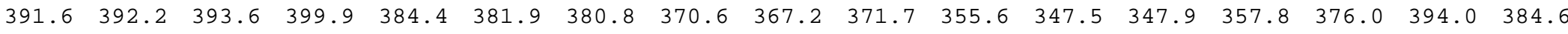

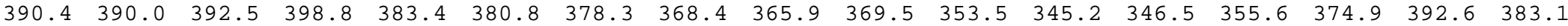

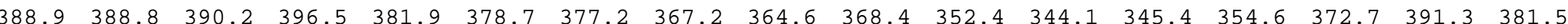

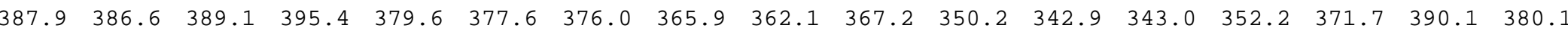

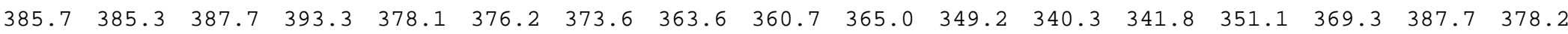

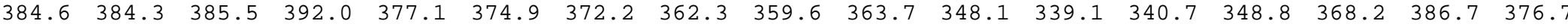

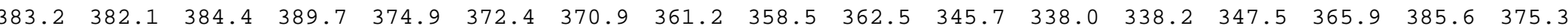

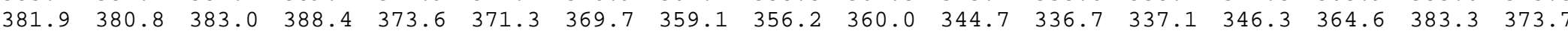

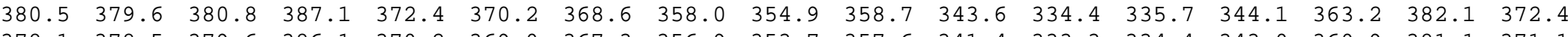

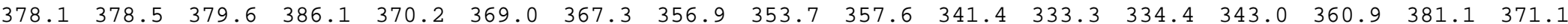

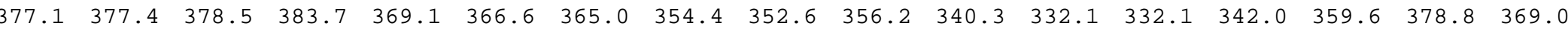

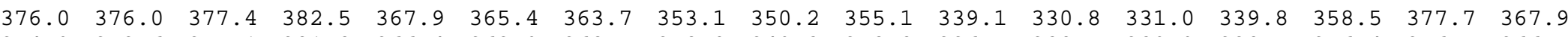

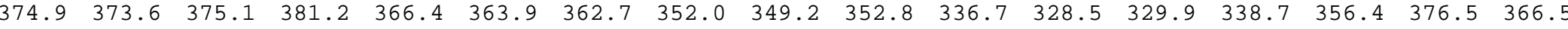

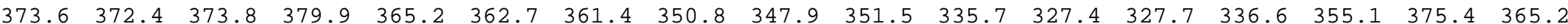

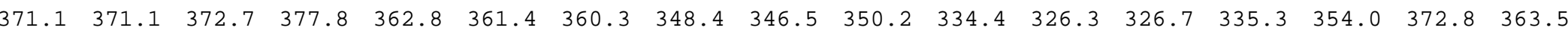

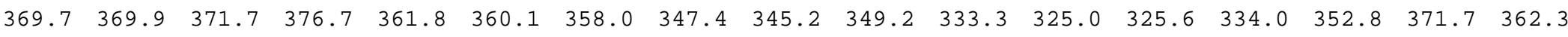

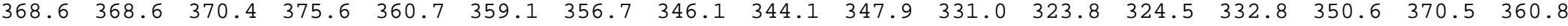

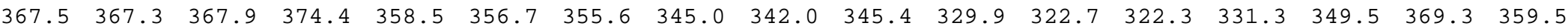

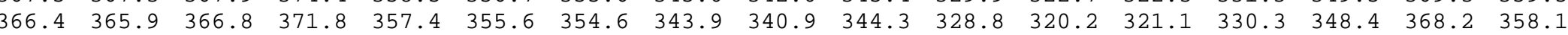
$\begin{array}{lllllllllllllllll}364.1 & 364.8 & 365.7 & 370.6 & 356.4 & 354.6 & 353.5 & 341.4 & 339.6 & 343.2 & 327.6 & 319.1 & 320.0 & 328.8 & 346.1 & 365.9 & 356.6\end{array}$

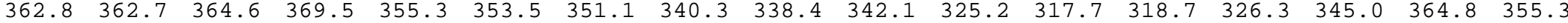

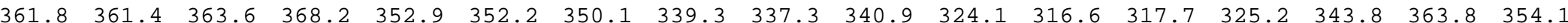

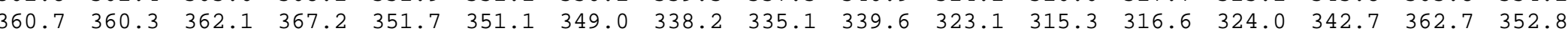

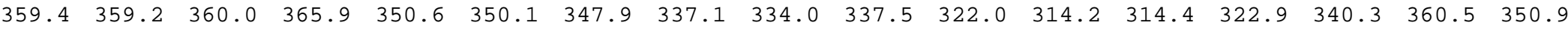

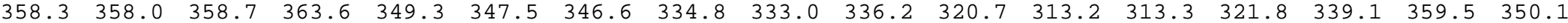

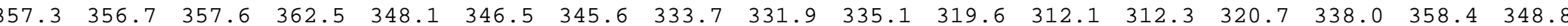

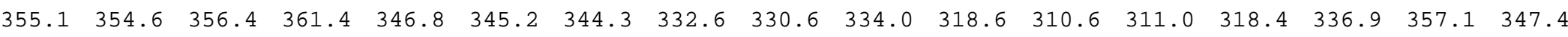
$\begin{array}{lllllllllllllllll}353.8 & 353.5 & 355.3 & 360.1 & 345.6 & 344.1 & 342.1 & 331.5 & 328.3 & 331.9 & 317.1 & 309.6 & 309.7 & 317.1 & 335.8 & 356.0 & 346.1\end{array}$

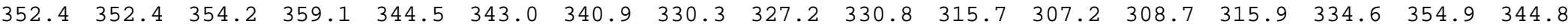

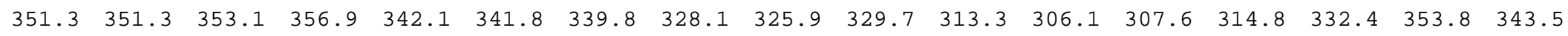


$\begin{array}{lllllllllllllllllll}350.2 & 350.2 & 351.7 & 355.8 & 341.1 & 340.5 & 338.5 & 327.0 & 324.9 & 328.3 & 313.3 & 305.1 & 306.5 & 313.5 & 331.3 & 352.5 & 342.3\end{array}$

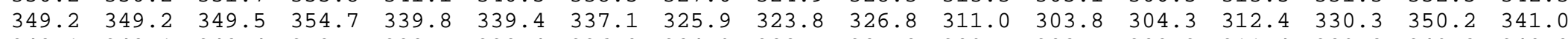

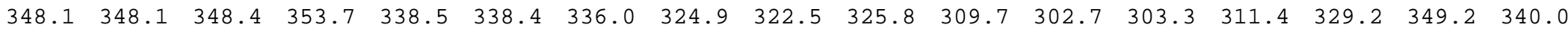

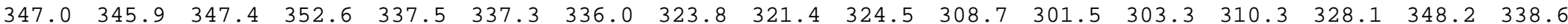

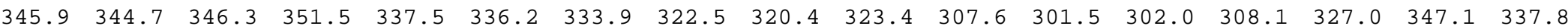

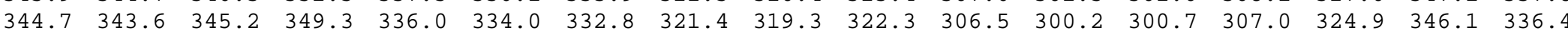

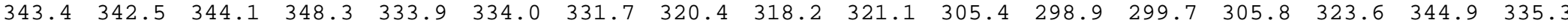

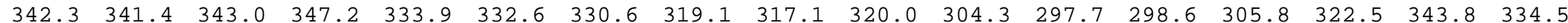

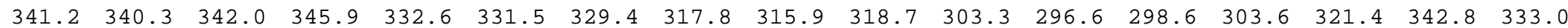

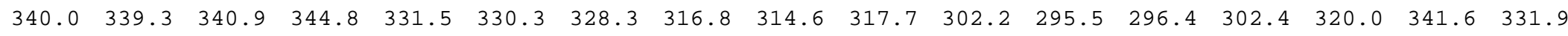

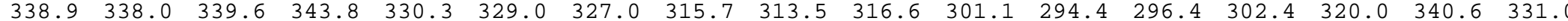

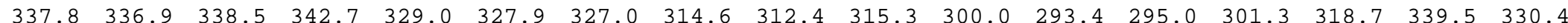

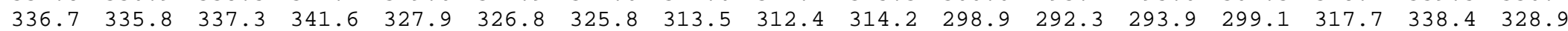

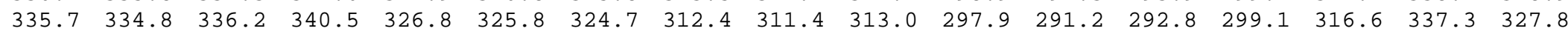

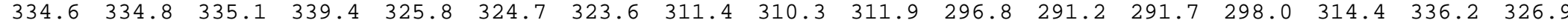

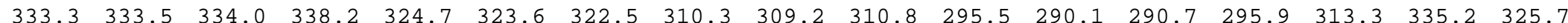

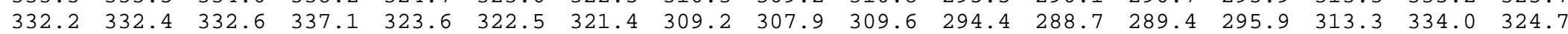

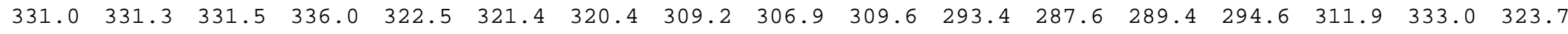

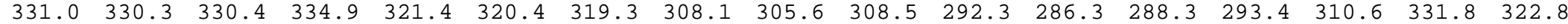

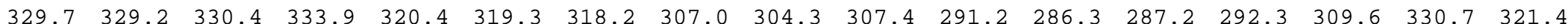

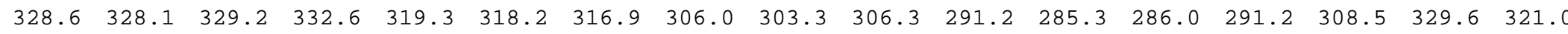

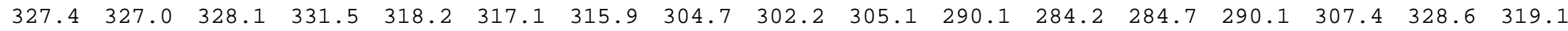

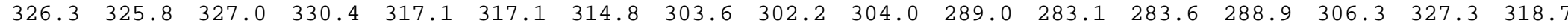

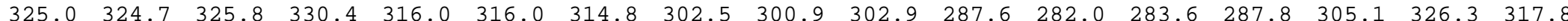

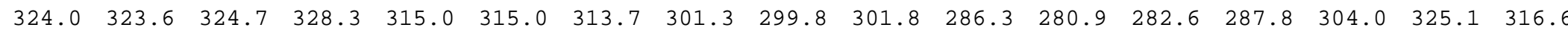

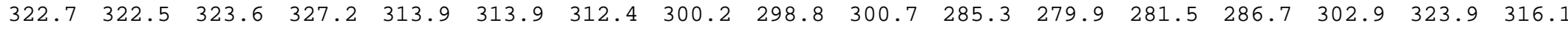

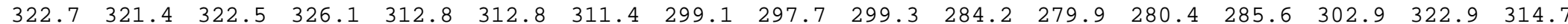

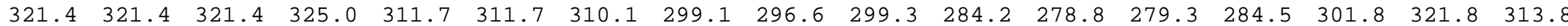

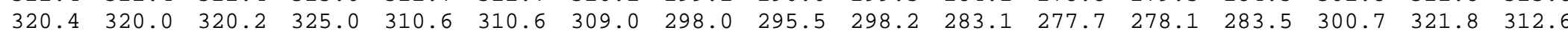

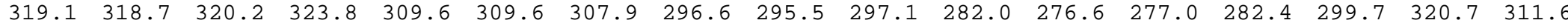

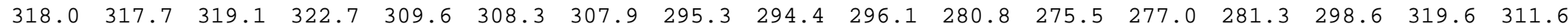

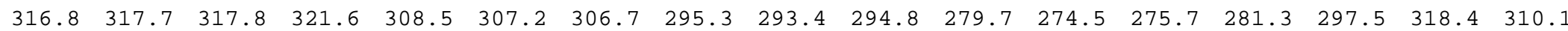

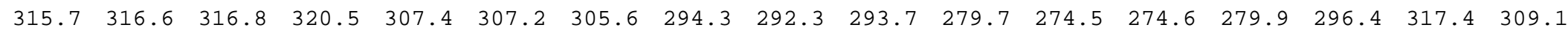

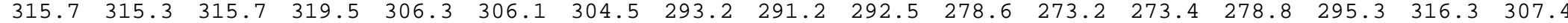

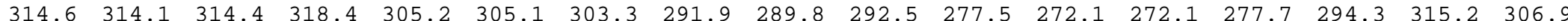

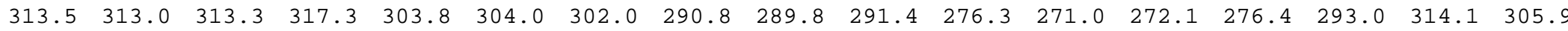

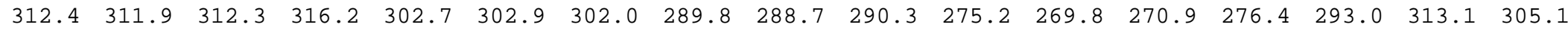

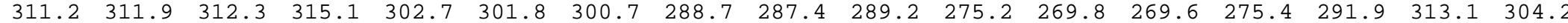

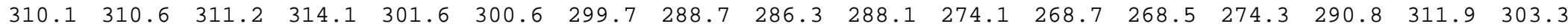

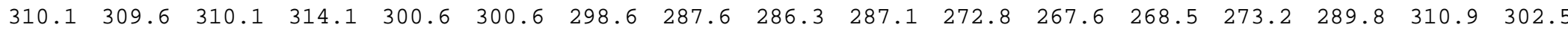

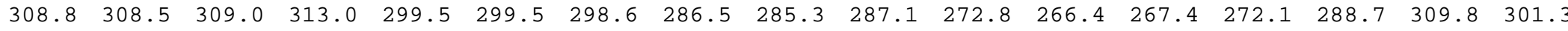

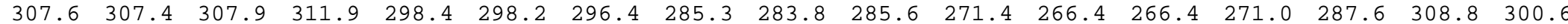

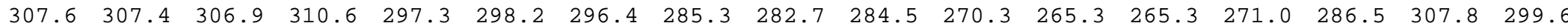

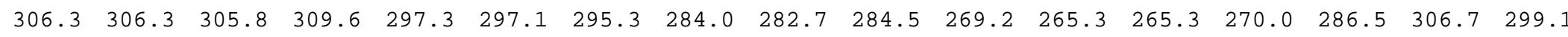

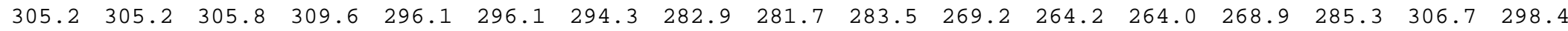

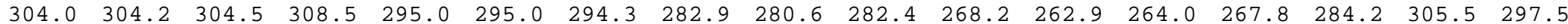

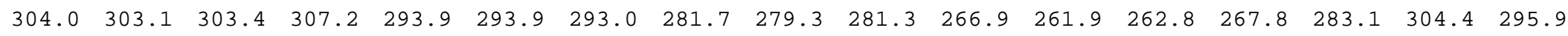


$\begin{array}{llllllllllllllllllll}302.7 & 302.0 & 303.4 & 306.1 & 293.9 & 293.9 & 291.9 & 280.6 & 279.3 & 280.2 & 266.9 & 261.9 & 261.7 & 266.7 & 283.1 & 303.4 & 295.6\end{array}$

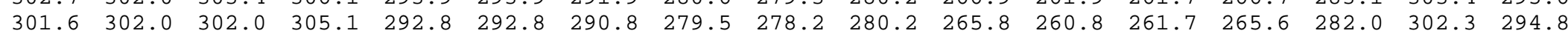

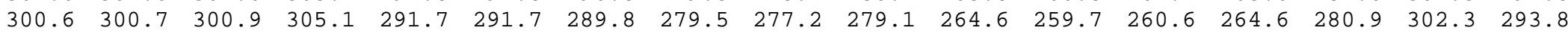

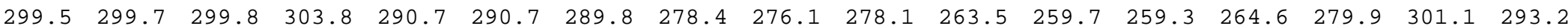

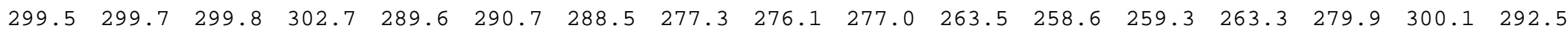

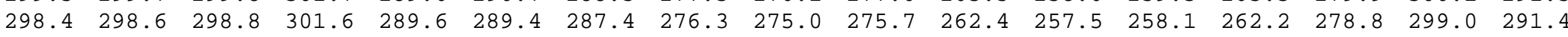

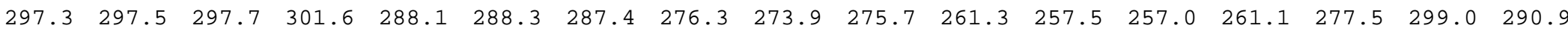

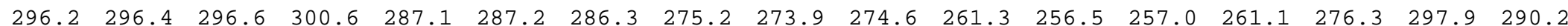

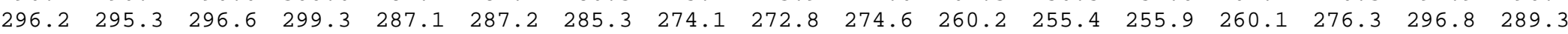

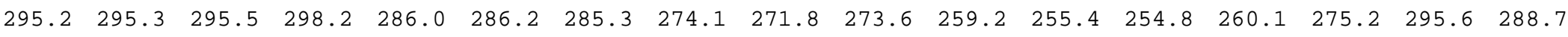

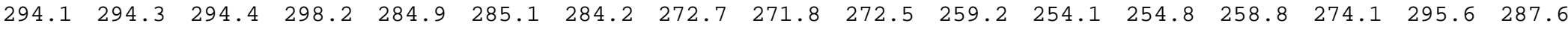

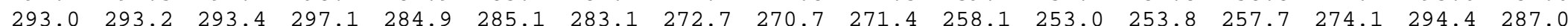

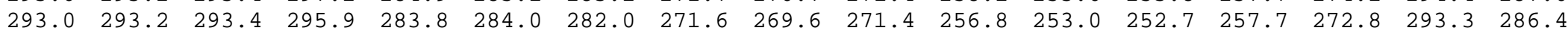

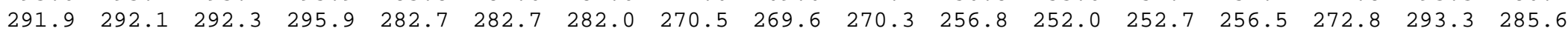

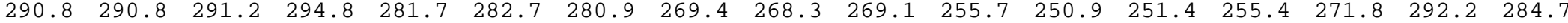

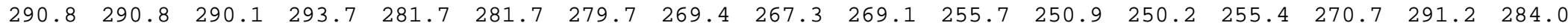

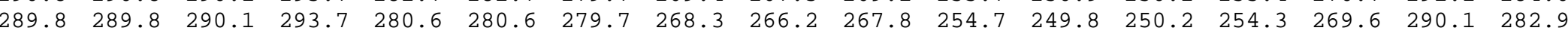

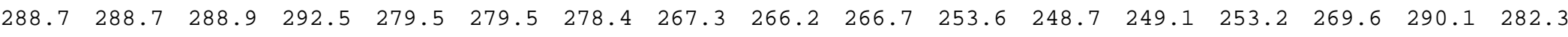

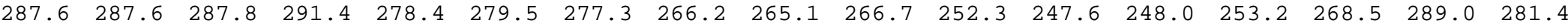

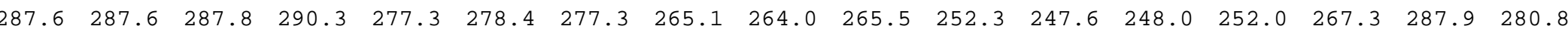

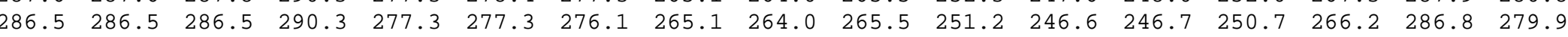

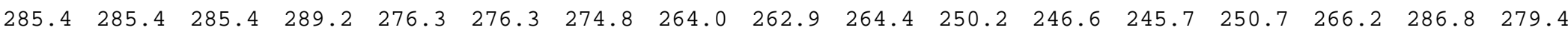

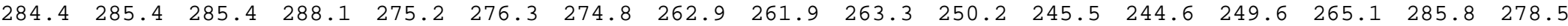

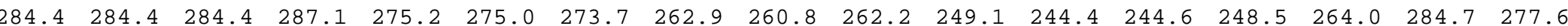

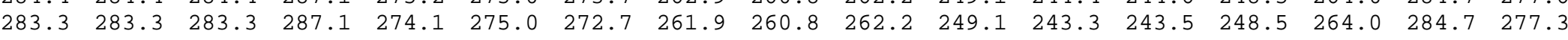

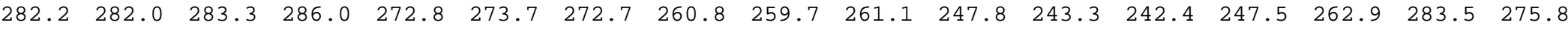

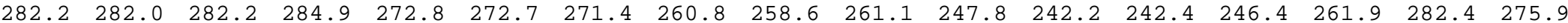

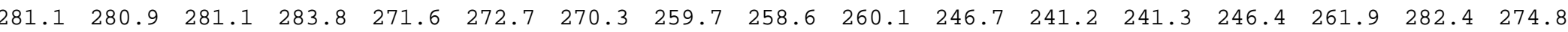

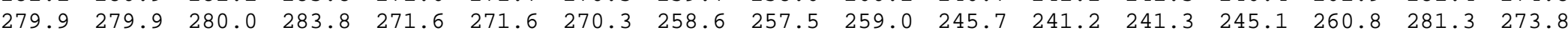

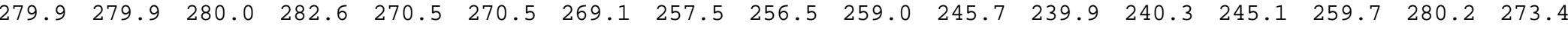

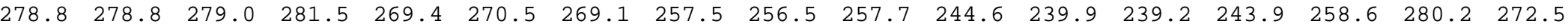

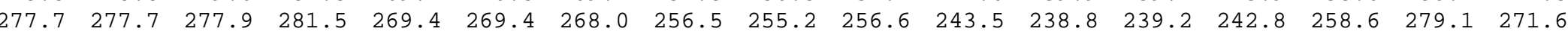

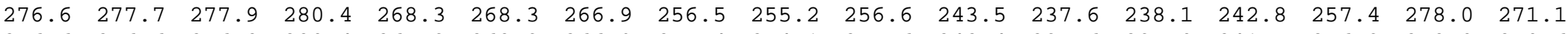

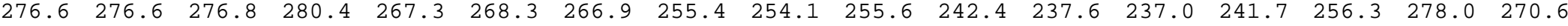

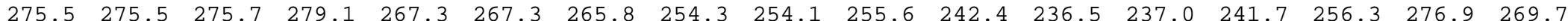

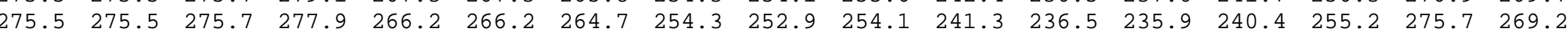

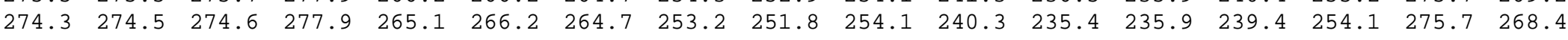

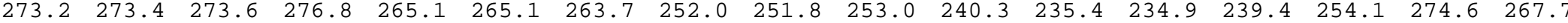

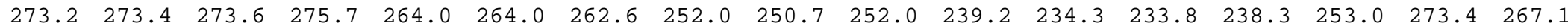
$\begin{array}{lllllllllllllllll}272.1 & 272.3 & 272.3 & 275.7 & 262.8 & 264.0 & 262.6 & 250.9 & 249.6 & 252.0 & 238.1 & 233.2 & 233.8 & 238.3 & 253.0 & 273.4 & 266.6\end{array}$

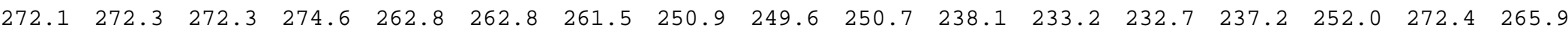

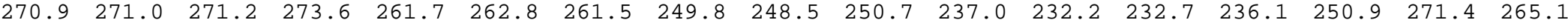

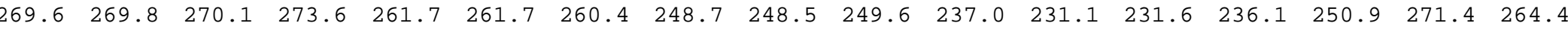

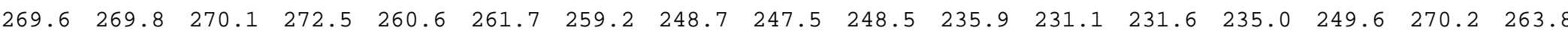

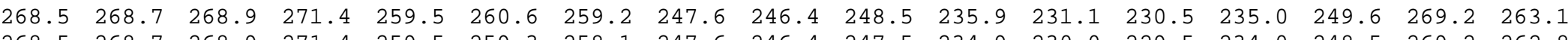

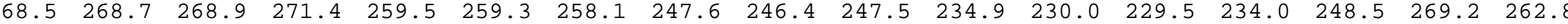

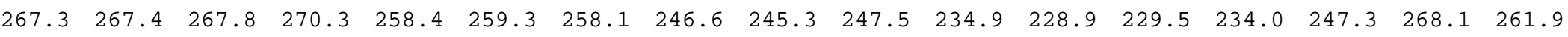


$\begin{array}{lllllllllllllllllll}266.2 & 267.4 & 266.7 & 269.2 & 257.4 & 258.3 & 257.0 & 245.5 & 245.3 & 246.4 & 233.8 & 228.9 & 228.4 & 232.9 & 247.3 & 268.1 & 260.9\end{array}$

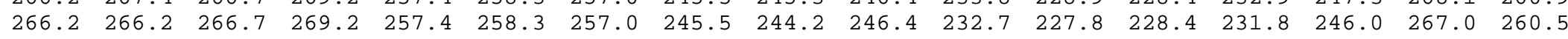

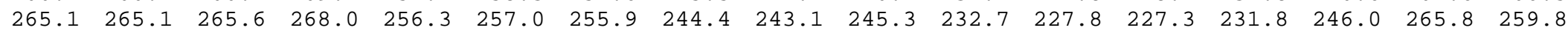

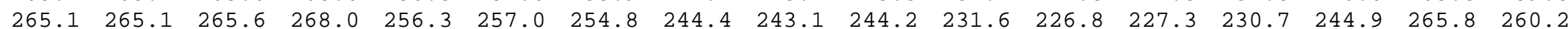

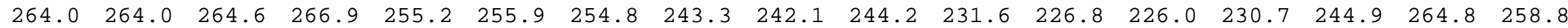

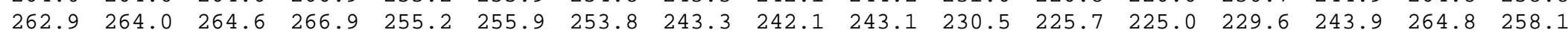

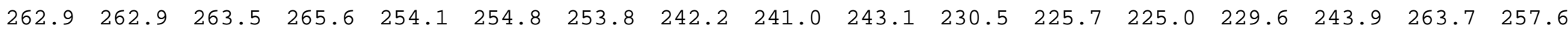

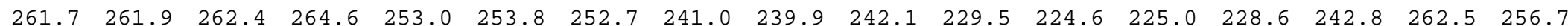

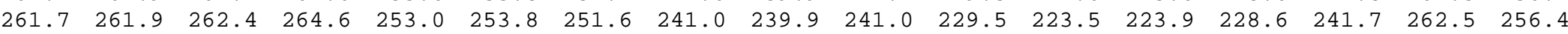

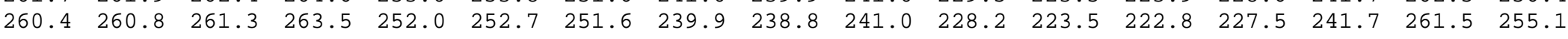

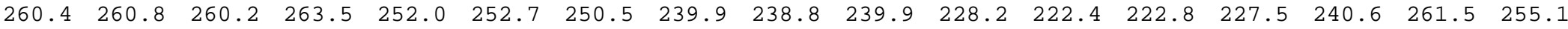

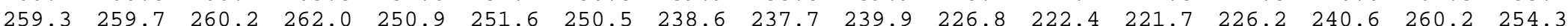

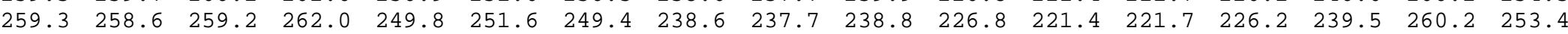

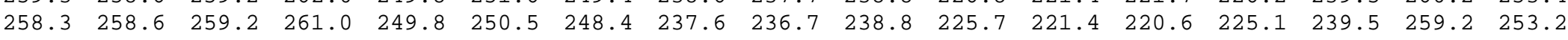

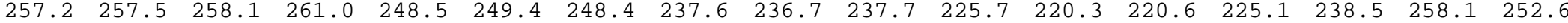

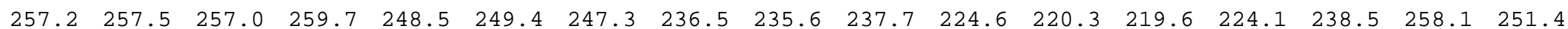

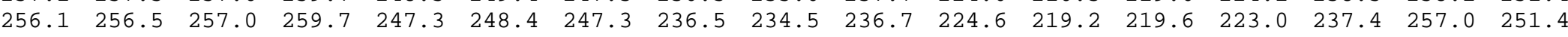

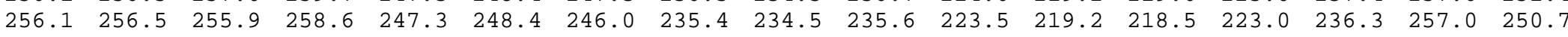

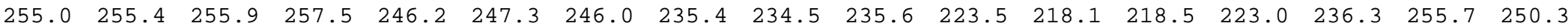

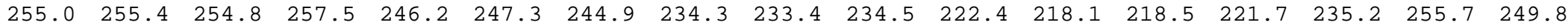

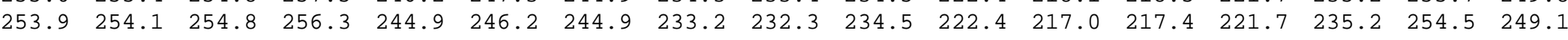

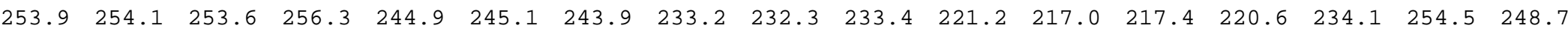

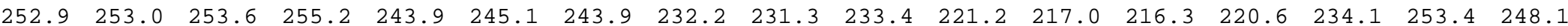

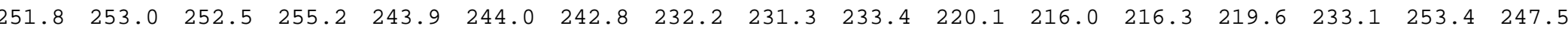

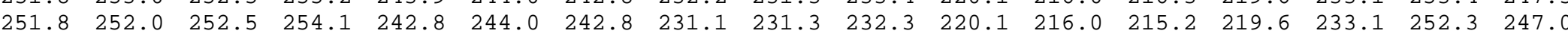

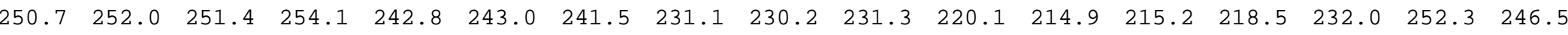

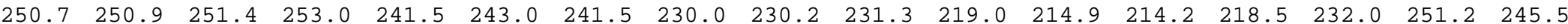

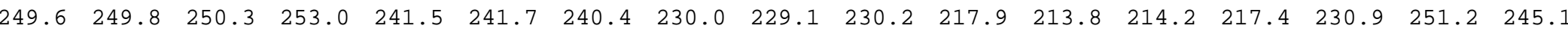

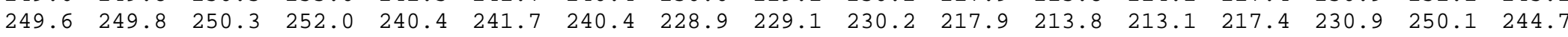

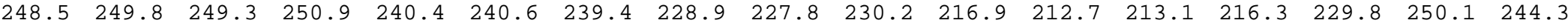

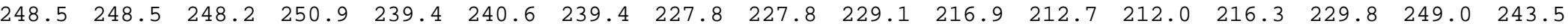

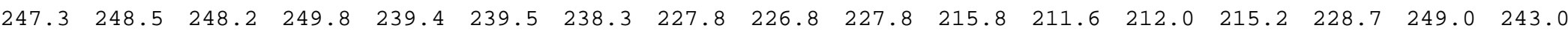

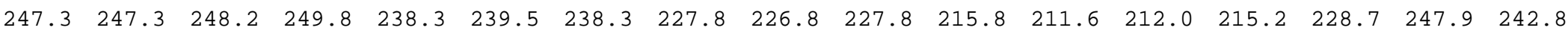

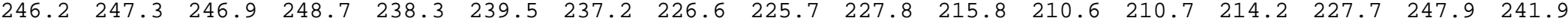

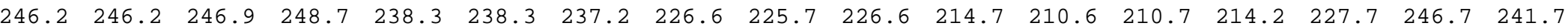

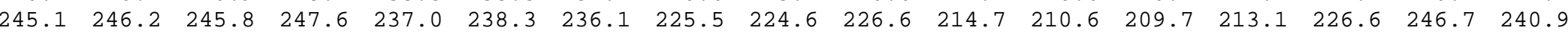

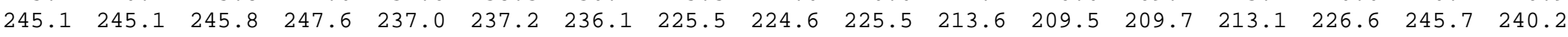

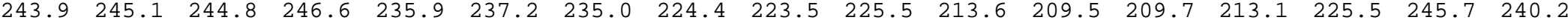

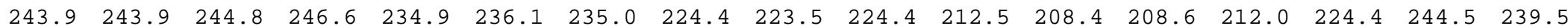

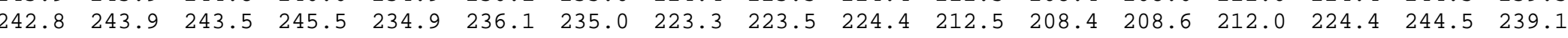

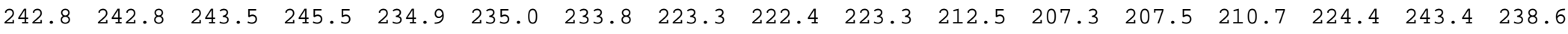

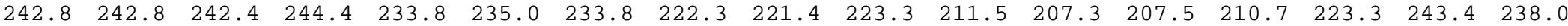

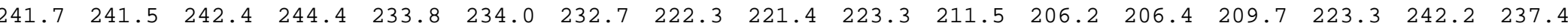

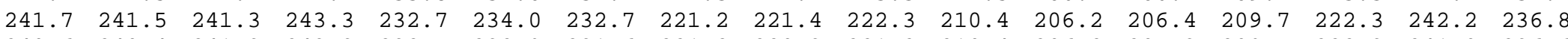

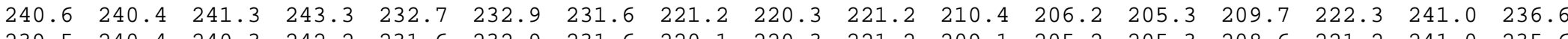

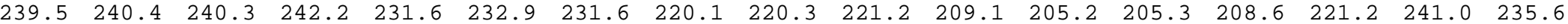

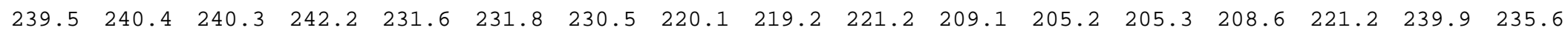


$\begin{array}{llllllllllllllllll}238.5 & 239.4 & 239.2 & 241.2 & 230.5 & 231.8 & 230.5 & 220.1 & 219.2 & 220.1 & 209.1 & 204.1 & 204.3 & 207.5 & 220.1 & 239.9 & 234.5\end{array}$

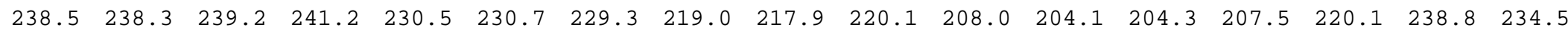

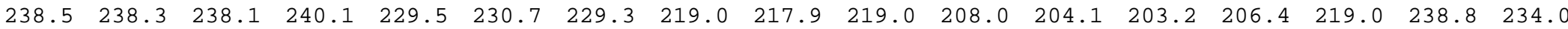

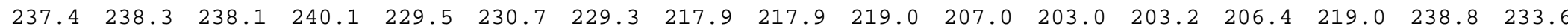

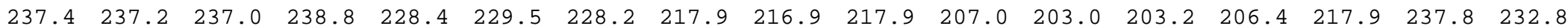

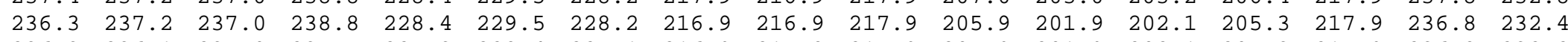

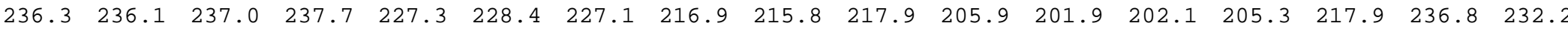

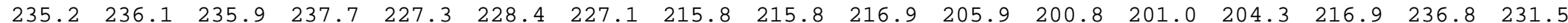

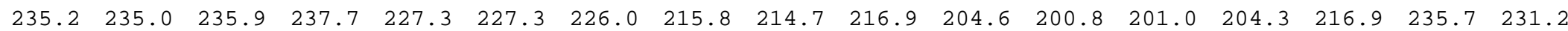

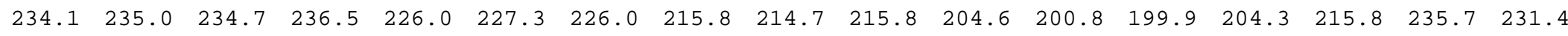

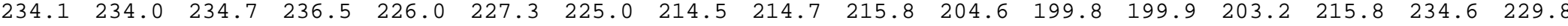

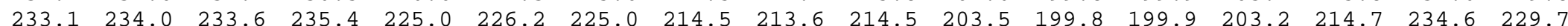

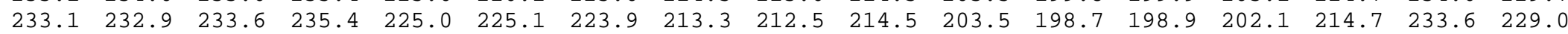

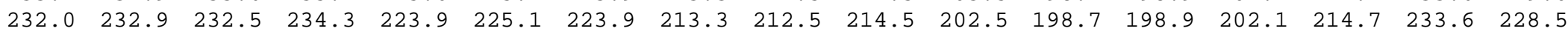

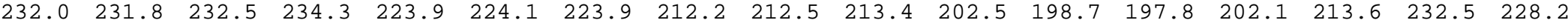

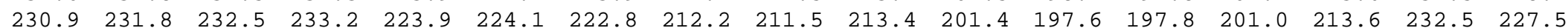

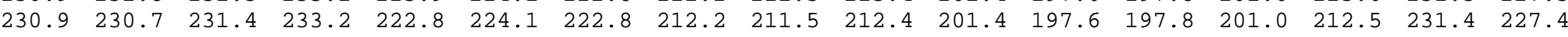

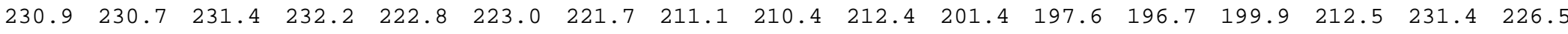

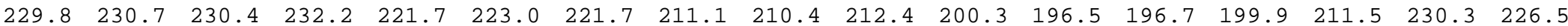

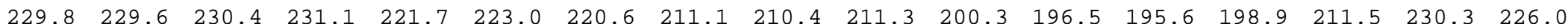

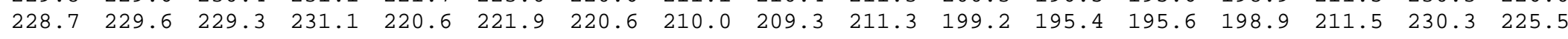

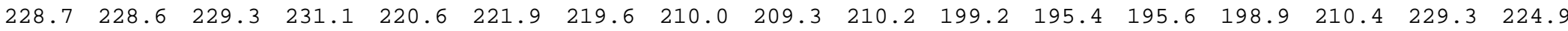

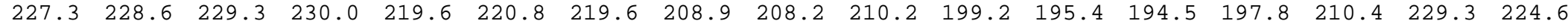

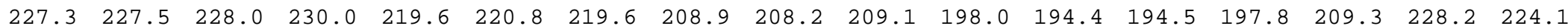

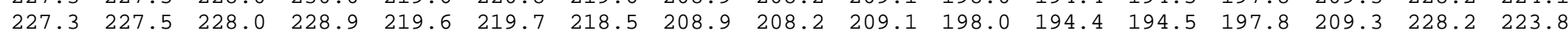

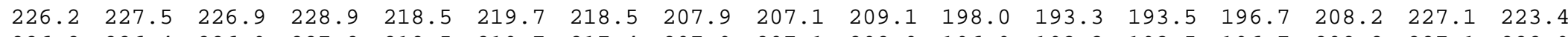

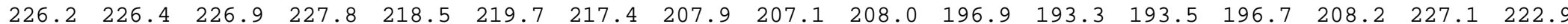

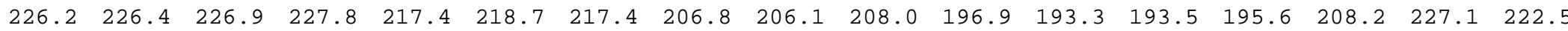

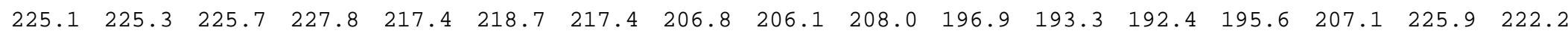

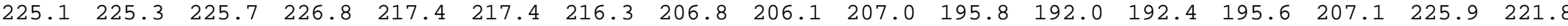

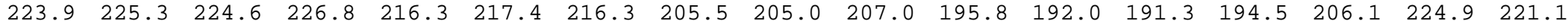

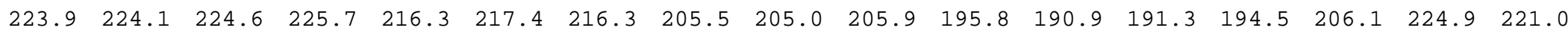

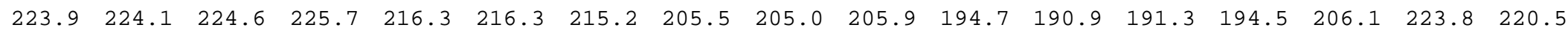

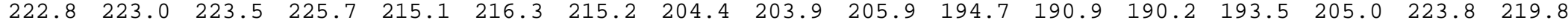

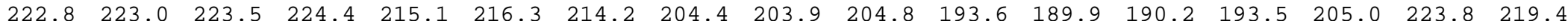

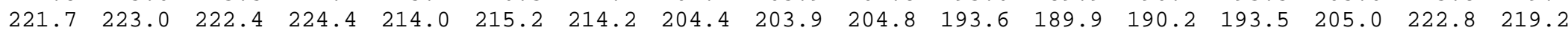

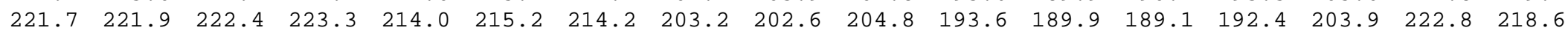

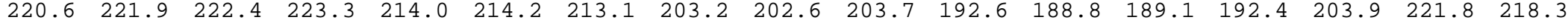

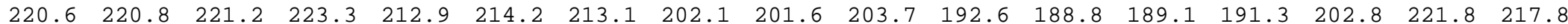

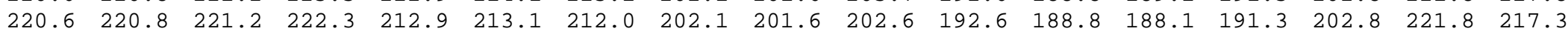

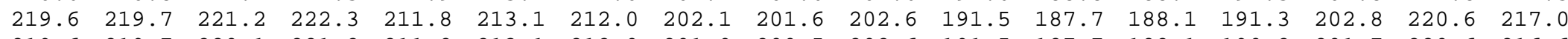

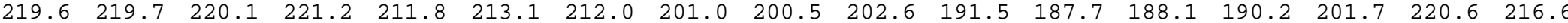

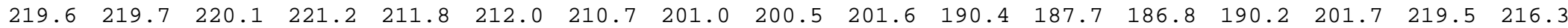

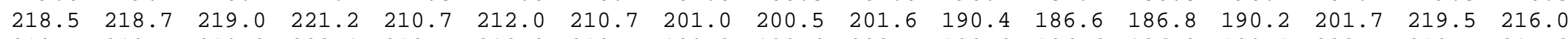

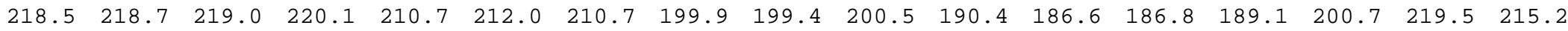

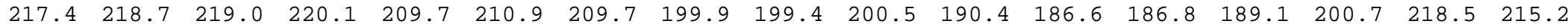

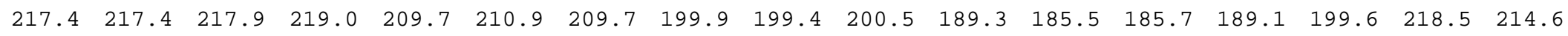


$\begin{array}{llllllllllllllllll}217.4 & 217.4 & 217.9 & 219.0 & 209.7 & 209.8 & 209.7 & 198.9 & 198.3 & 199.4 & 189.3 & 185.5 & 185.7 & 188.1 & 199.6 & 217.4 & 214.2\end{array}$

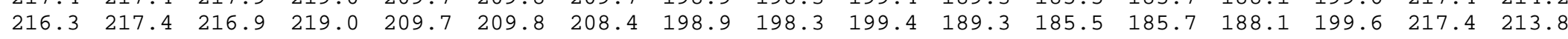

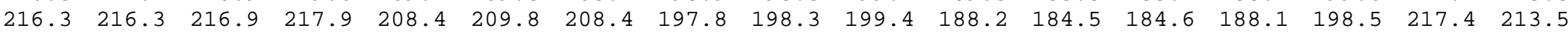

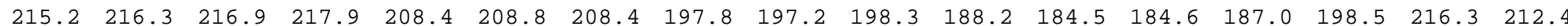

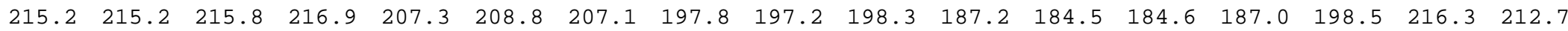

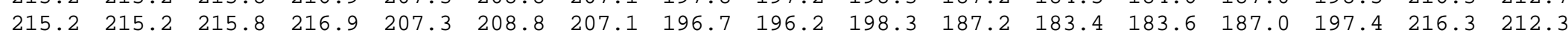

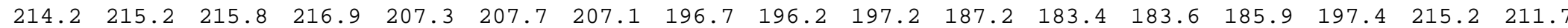

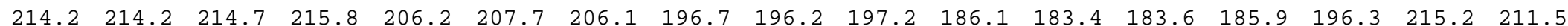

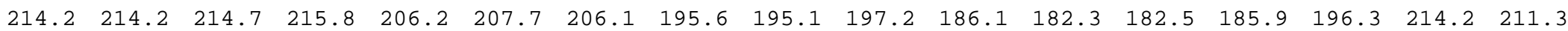

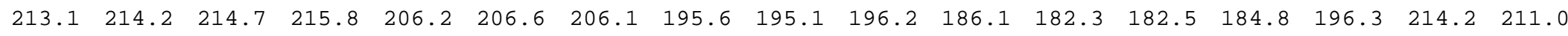

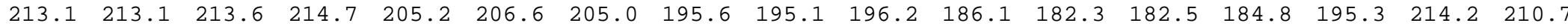

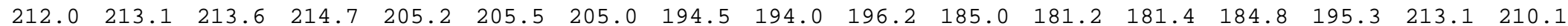

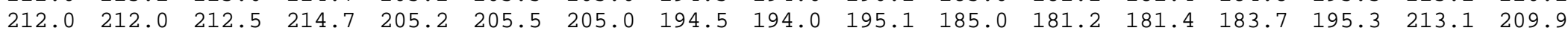

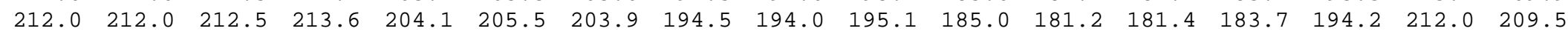

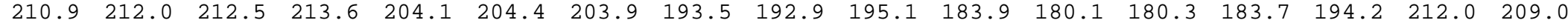

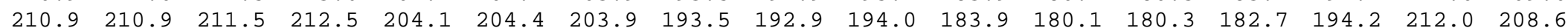

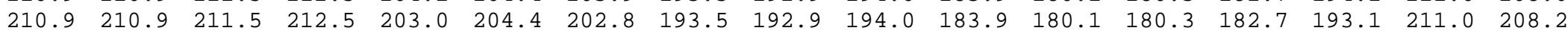

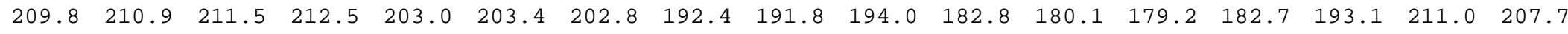

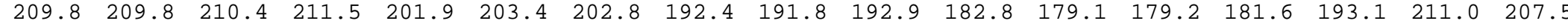

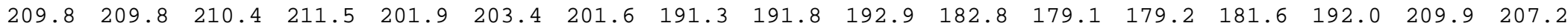

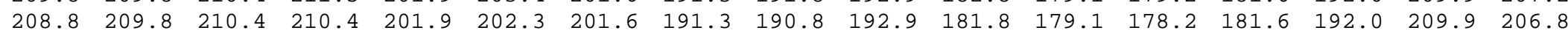

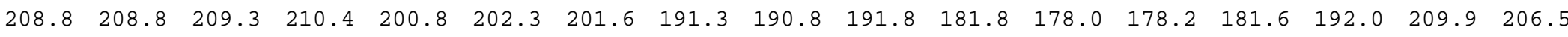

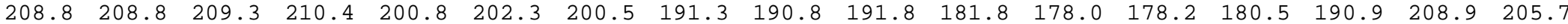

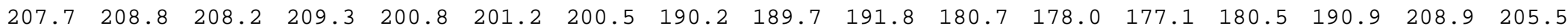

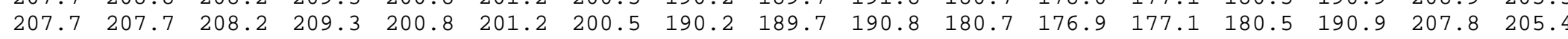

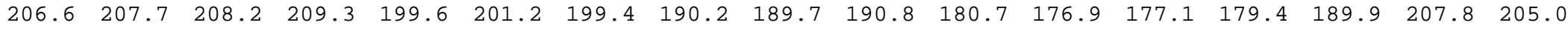

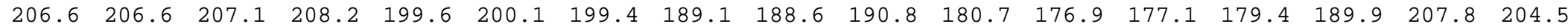

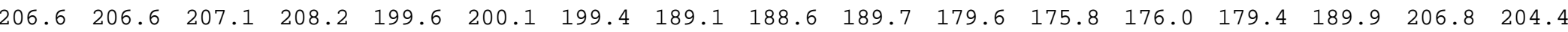

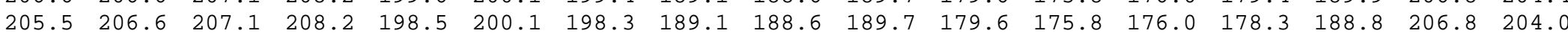

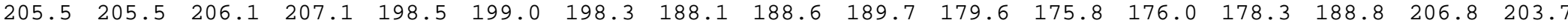

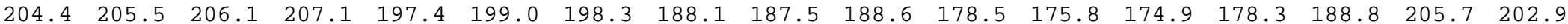

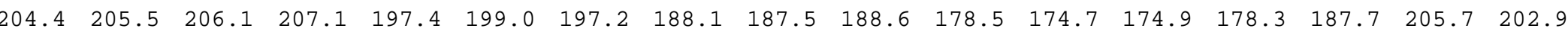

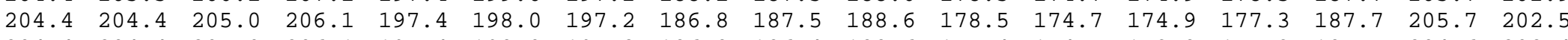

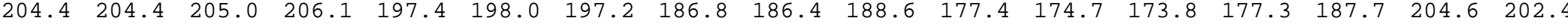

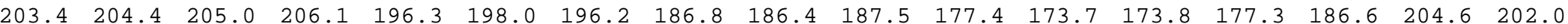

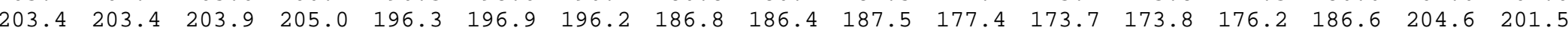

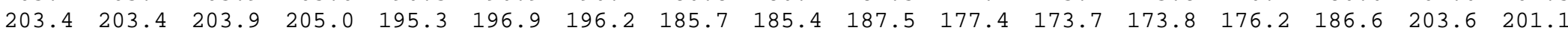

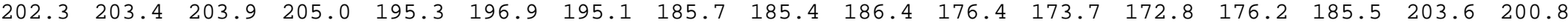

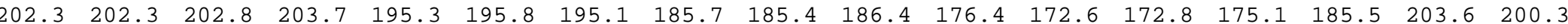

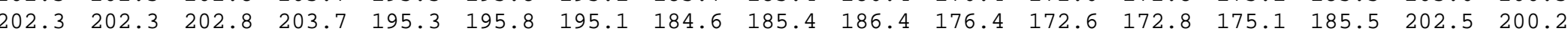

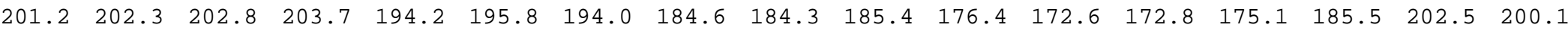

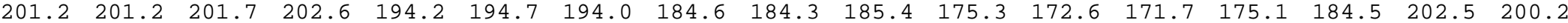

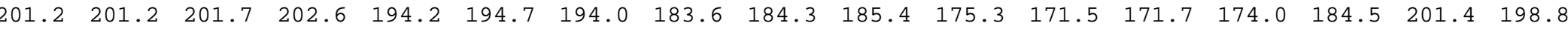

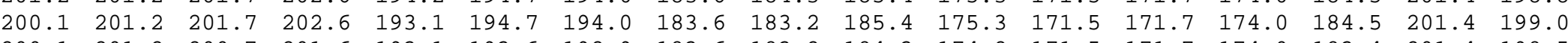

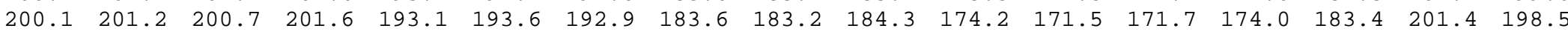

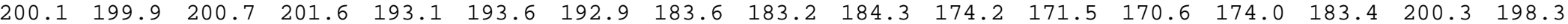

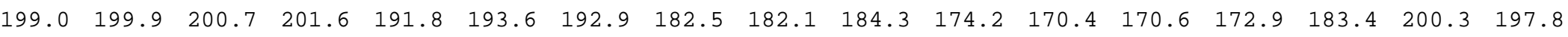


$\begin{array}{lllllllllllllllll}199.0 & 199.9 & 200.7 & 201.6 & 191.8 & 193.6 & 191.8 & 182.5 & 182.1 & 183.2 & 174.2 & 170.4 & 170.6 & 172.9 & 182.3 & 200.3 & 197.5\end{array}$

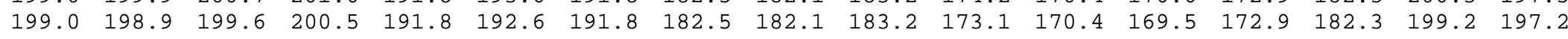

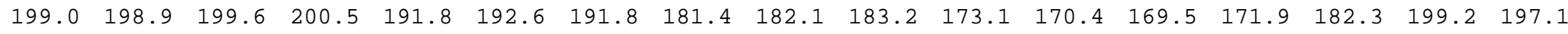

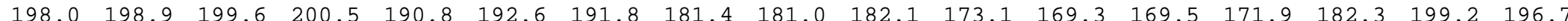

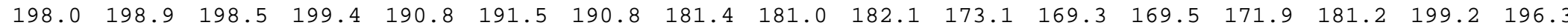

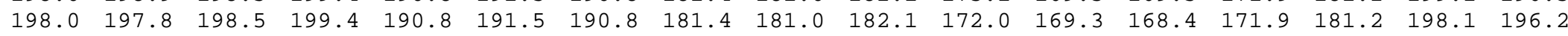

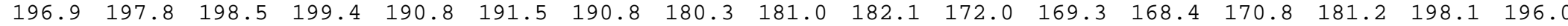

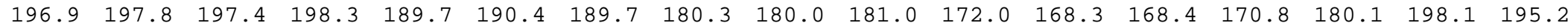

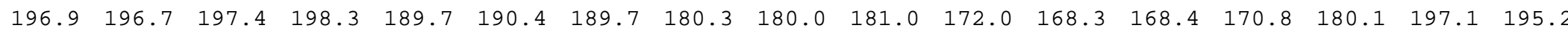

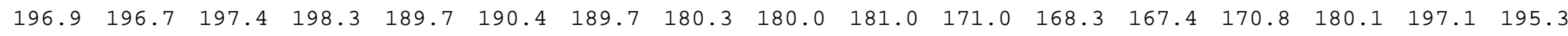

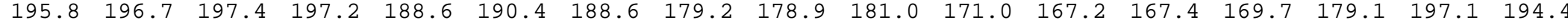

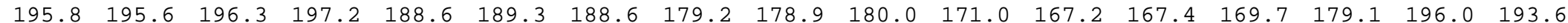

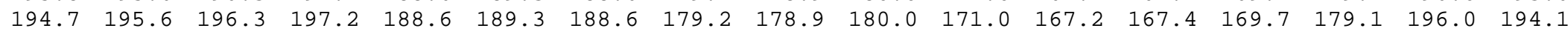

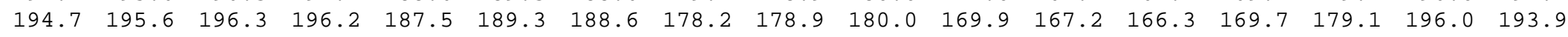

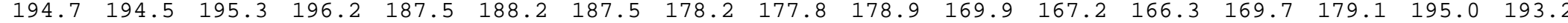

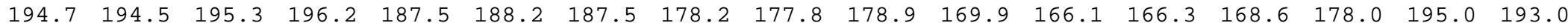

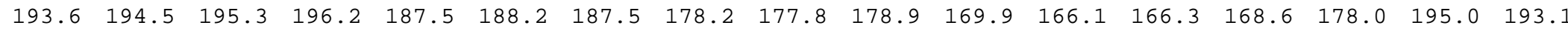

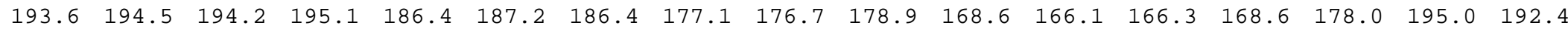

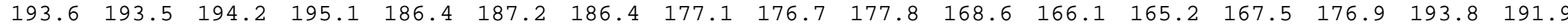

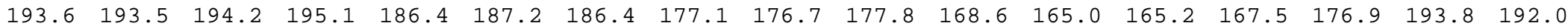

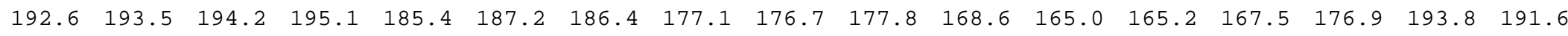

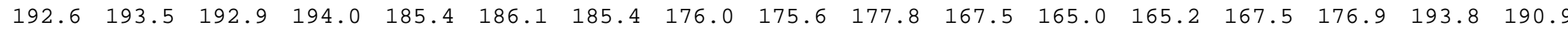

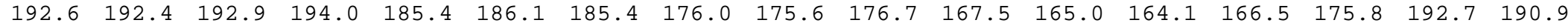

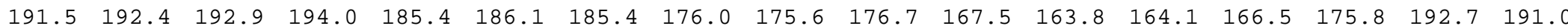

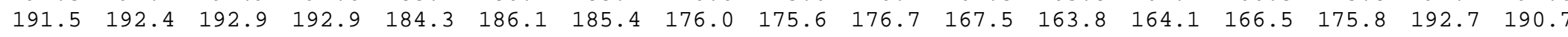

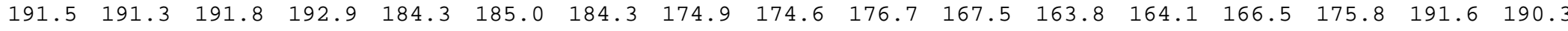

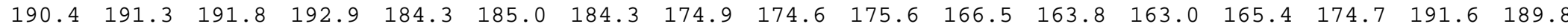

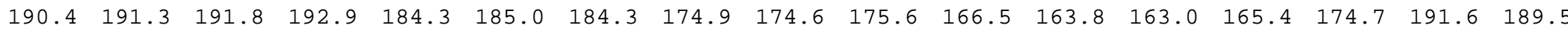

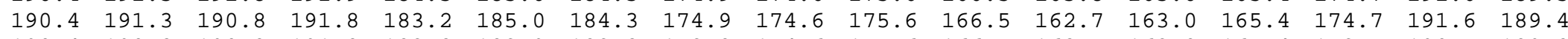

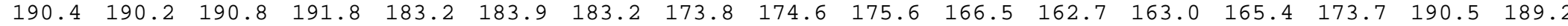

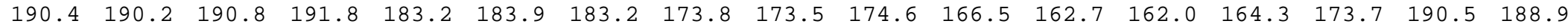

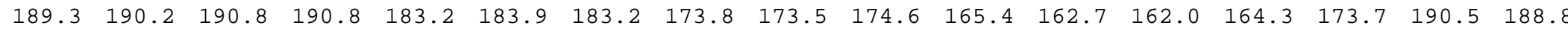

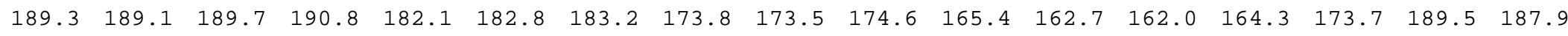

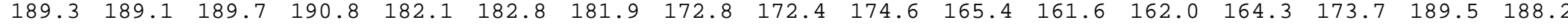

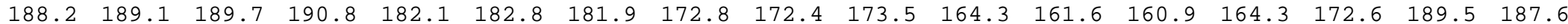

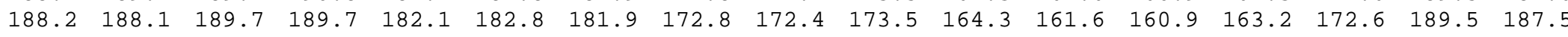

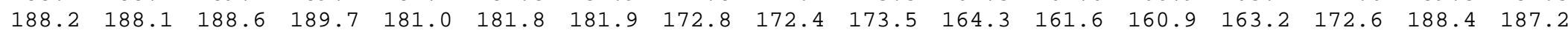

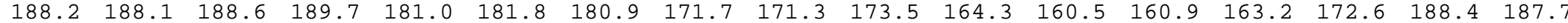

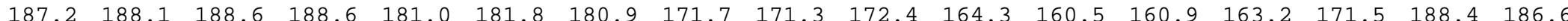

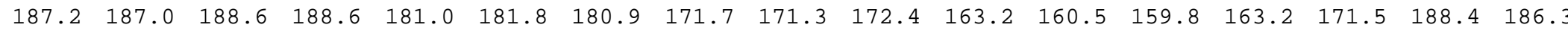

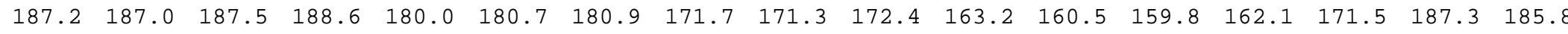

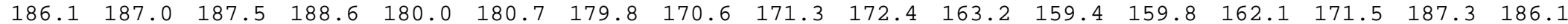

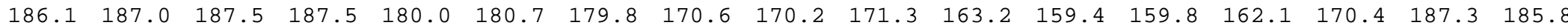

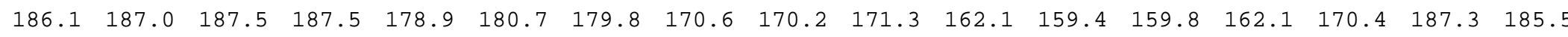

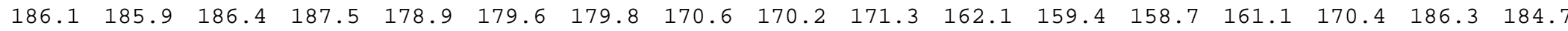

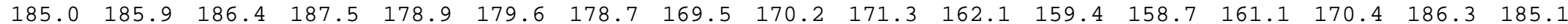

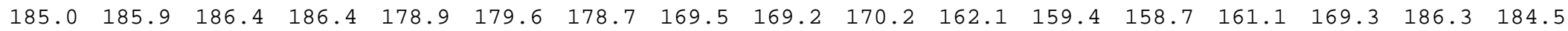




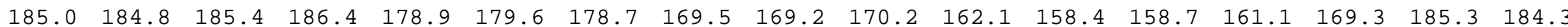

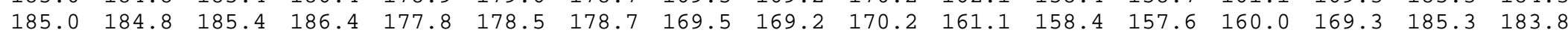

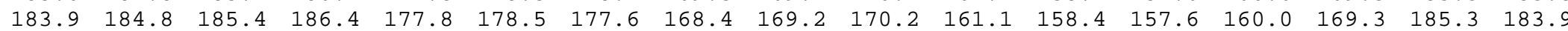

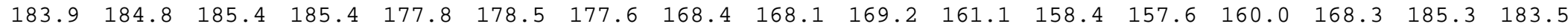

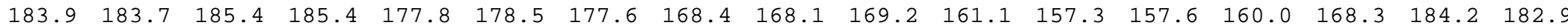

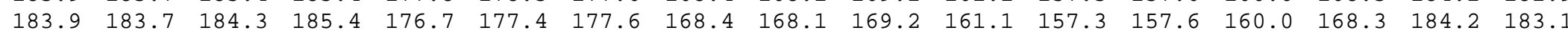

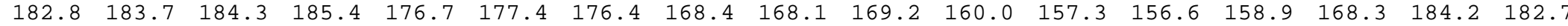

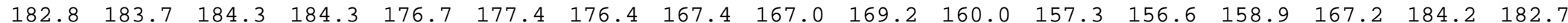

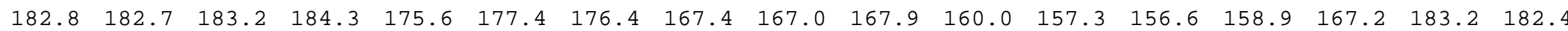

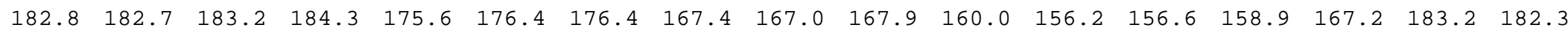

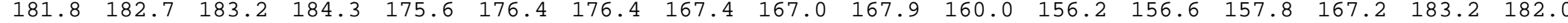

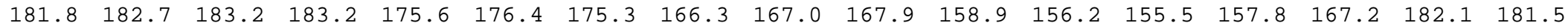

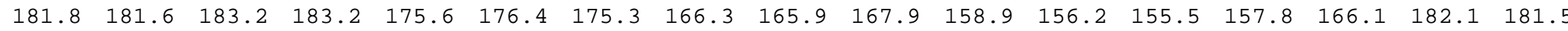

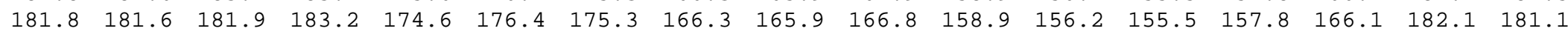

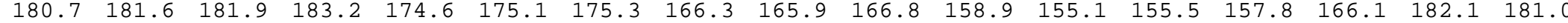

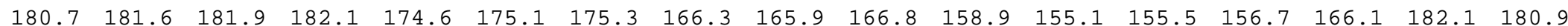

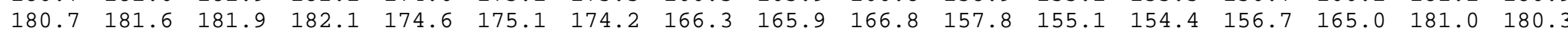

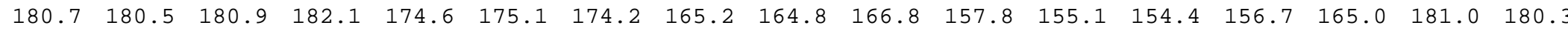

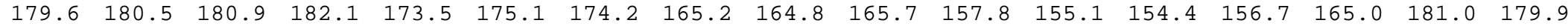

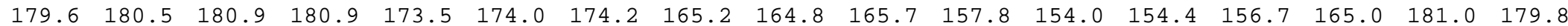

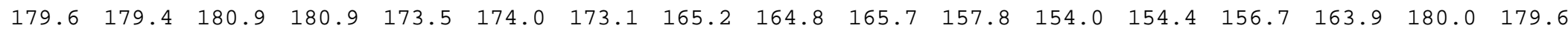

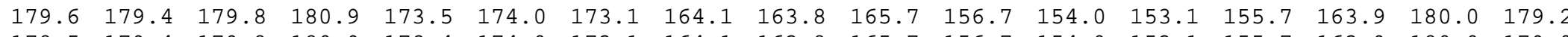

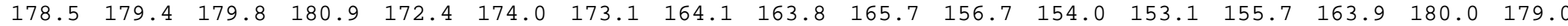

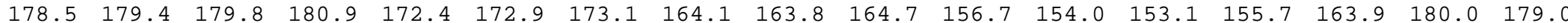

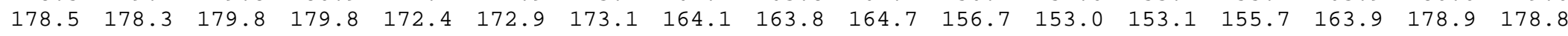

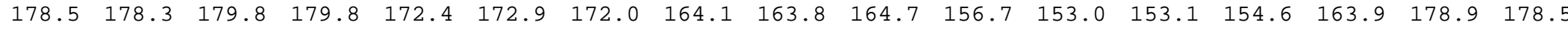

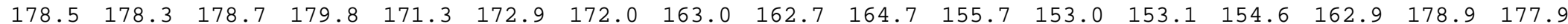

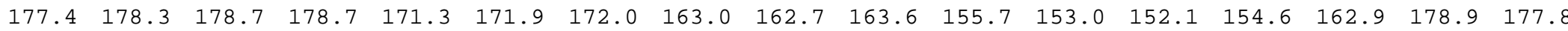

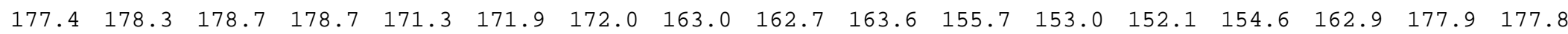

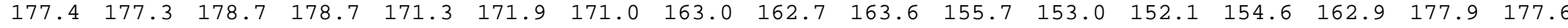

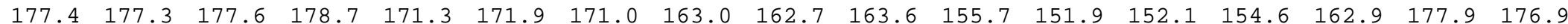

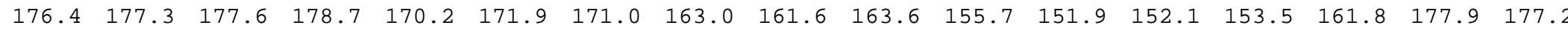

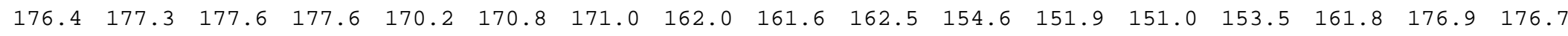

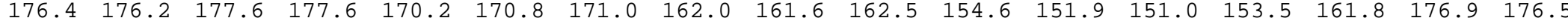

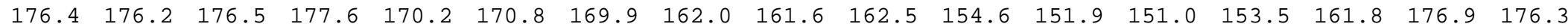

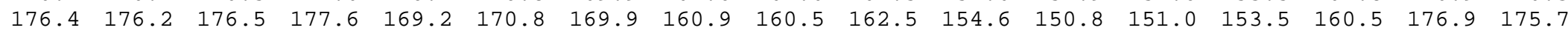

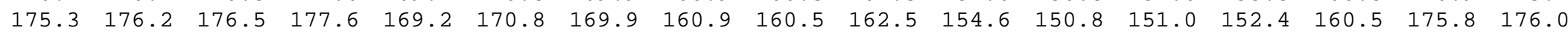

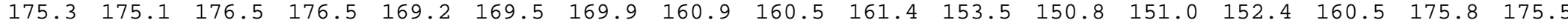

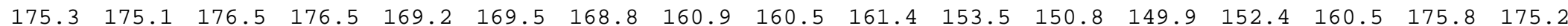

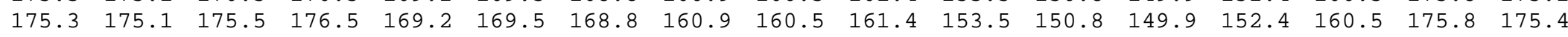

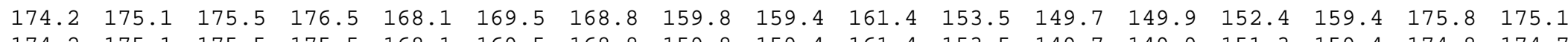

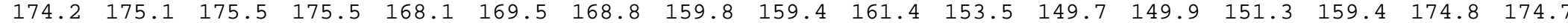

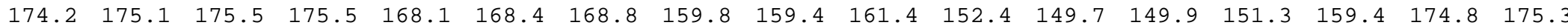

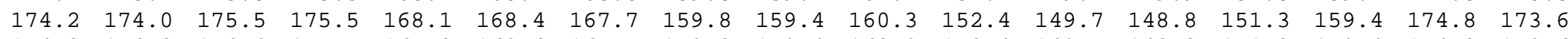

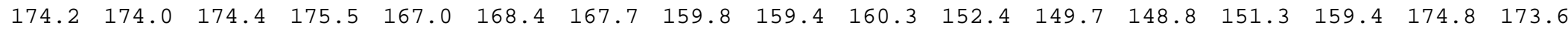

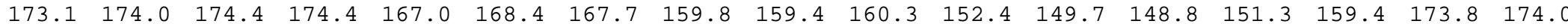

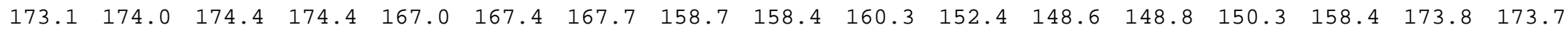


$\begin{array}{lllllllllllllllllll}173.1 & 172.8 & 174.4 & 174.4 & 167.0 & 167.4 & 167.7 & 158.7 & 158.4 & 160.3 & 152.4 & 148.6 & 148.8 & 150.3 & 158.4 & 173.8 & 173.5\end{array}$

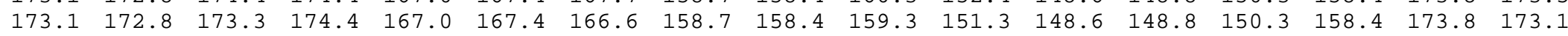

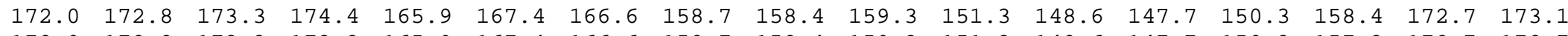

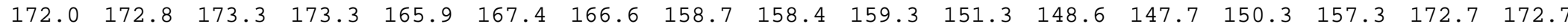

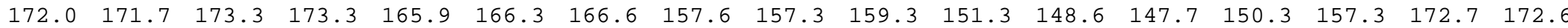

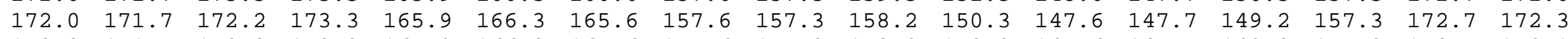

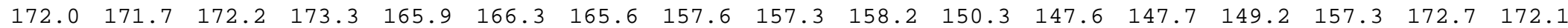

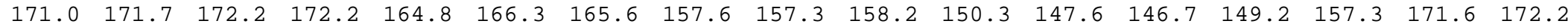

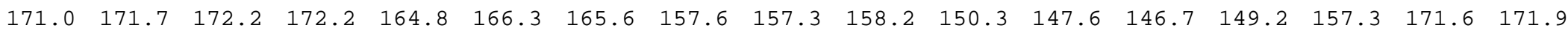

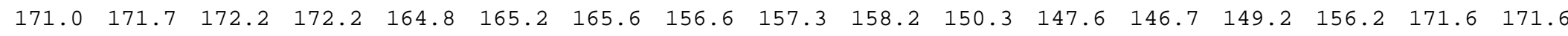

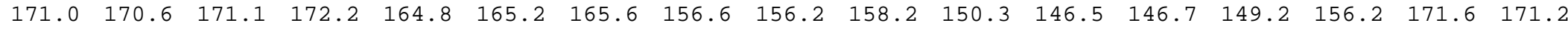

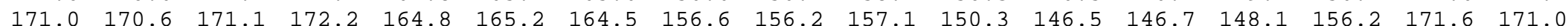

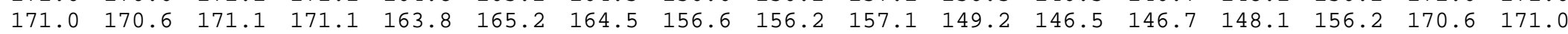

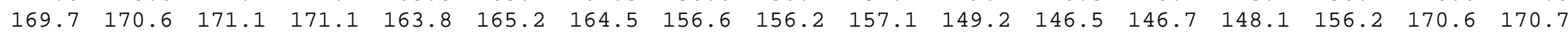

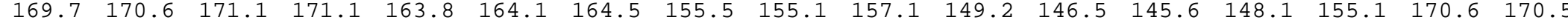

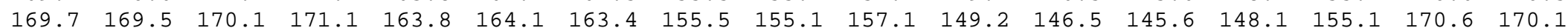

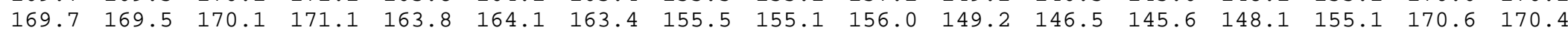

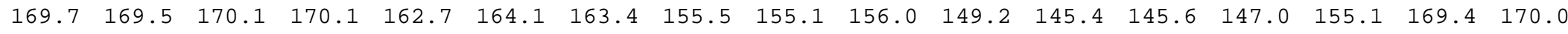

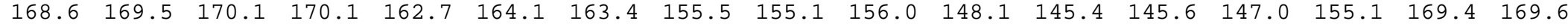

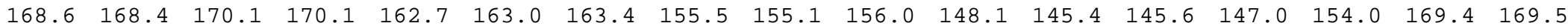

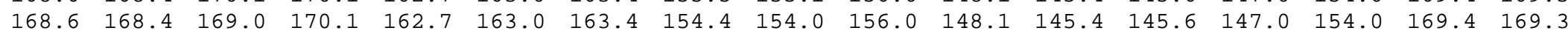

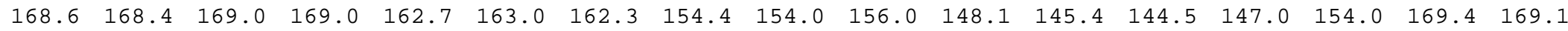

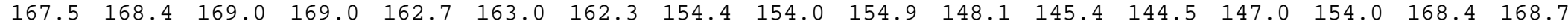

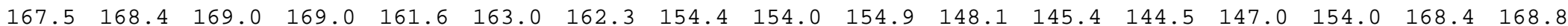

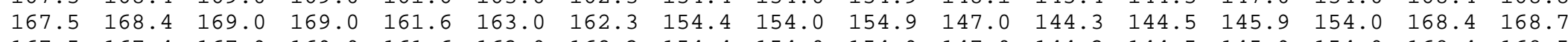

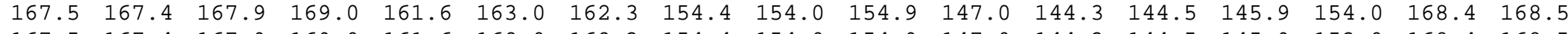

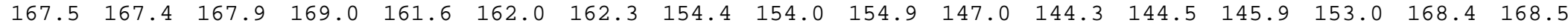

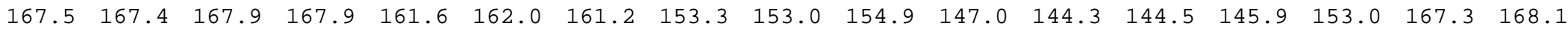

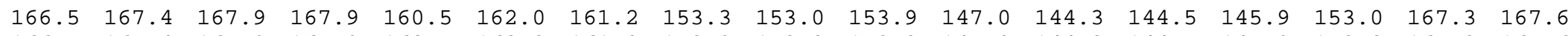

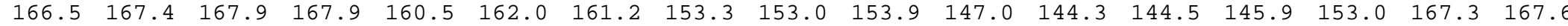

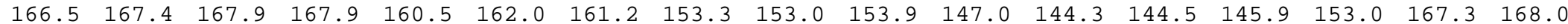

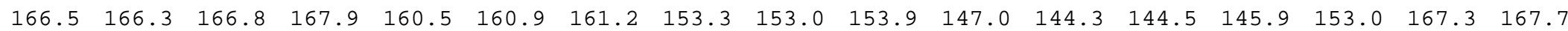

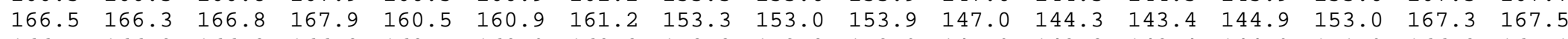

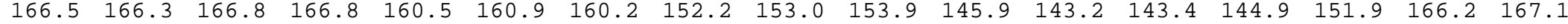

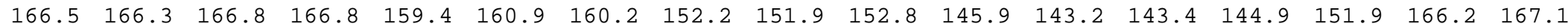

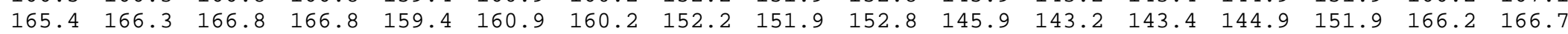

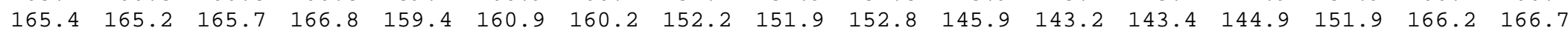

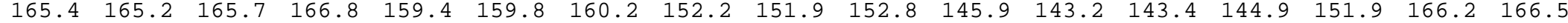

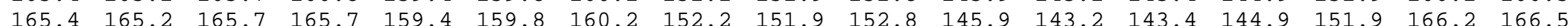

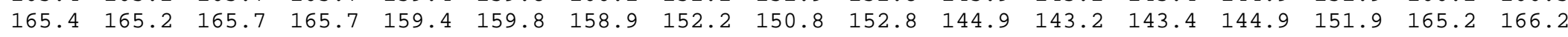

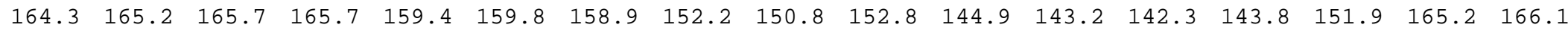

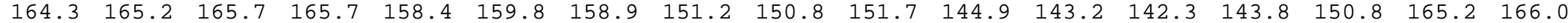

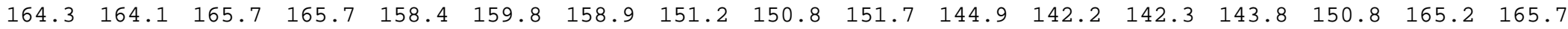

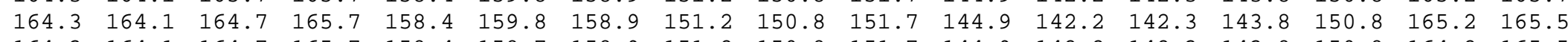

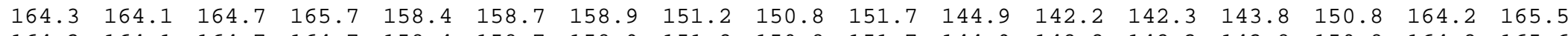

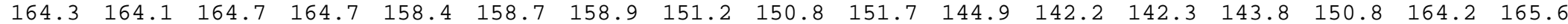

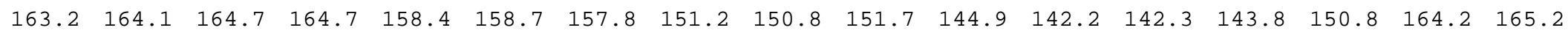




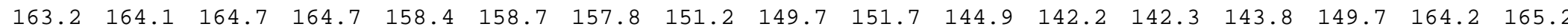

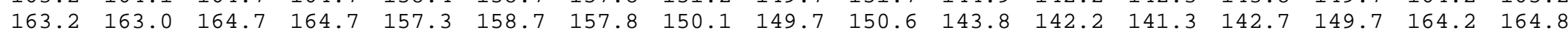

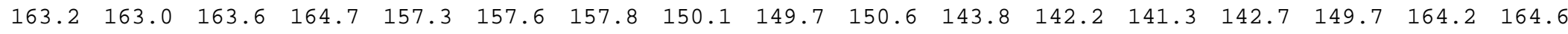

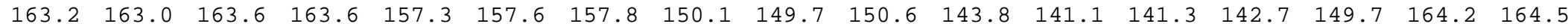

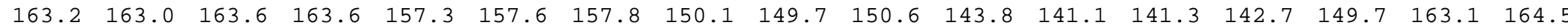

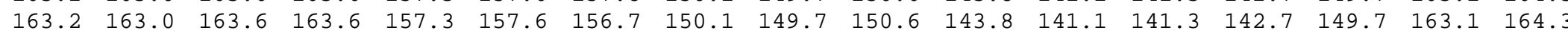

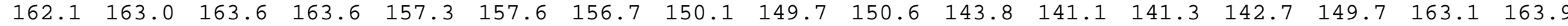

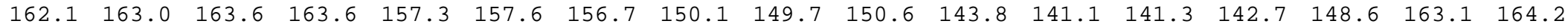

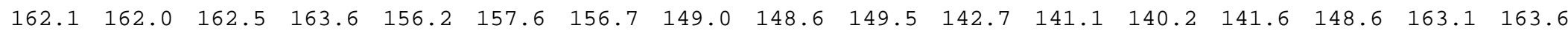

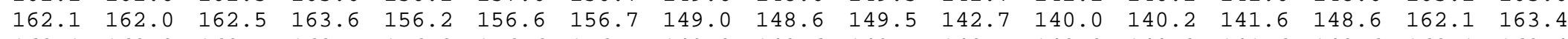

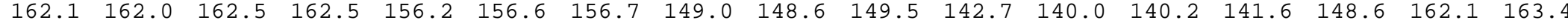

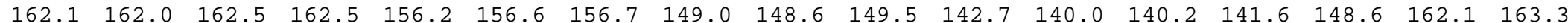

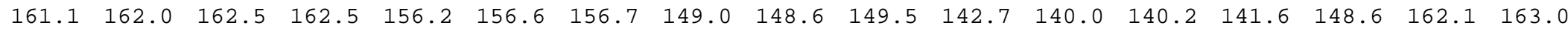

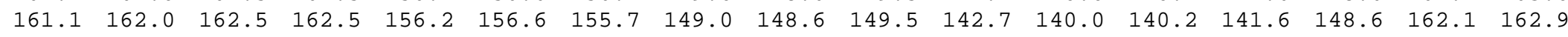

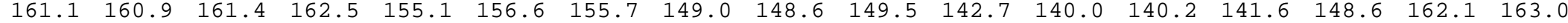

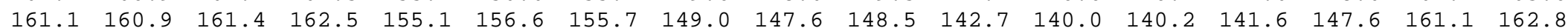

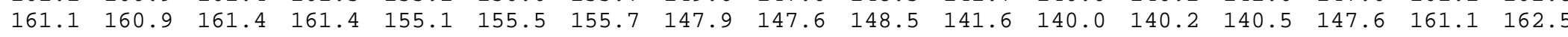

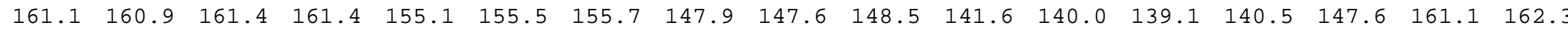

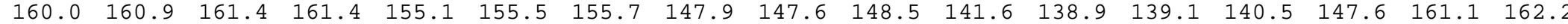

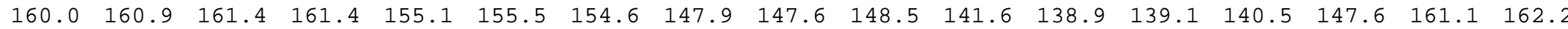

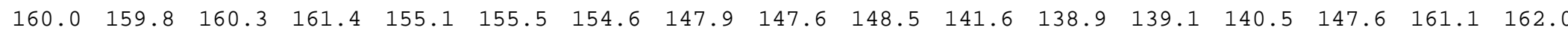

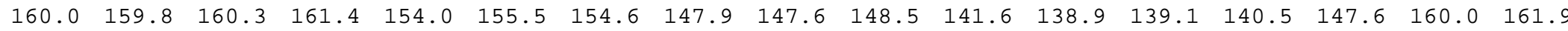

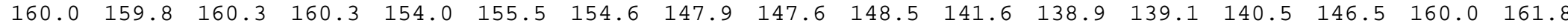

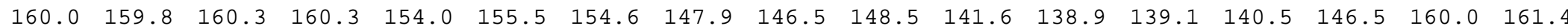

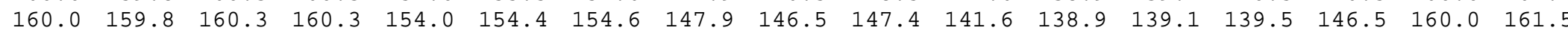

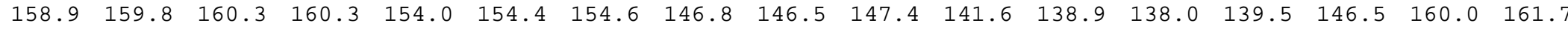

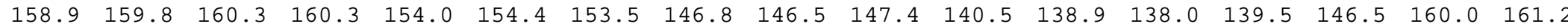

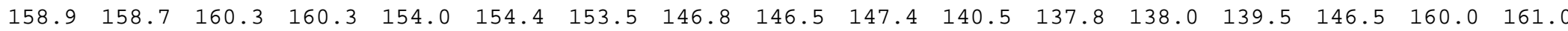

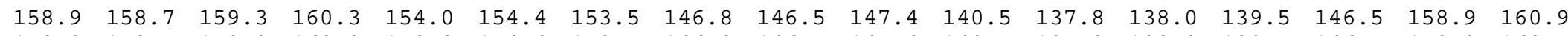

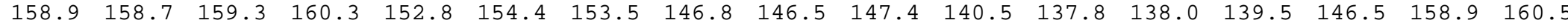

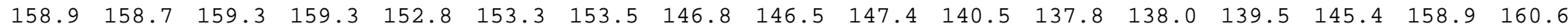

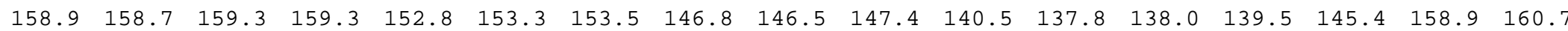

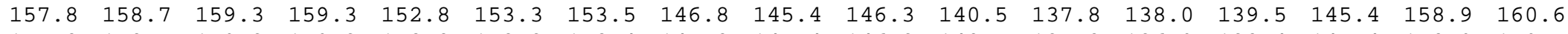

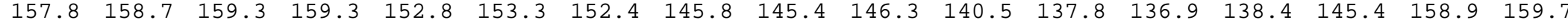

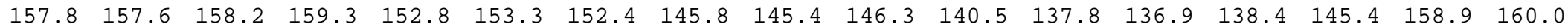

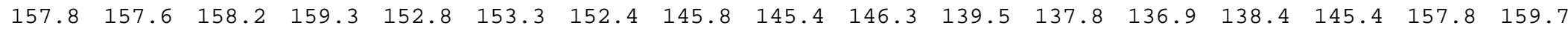

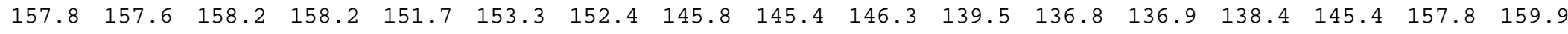

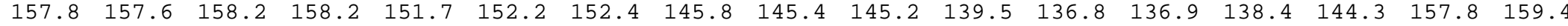

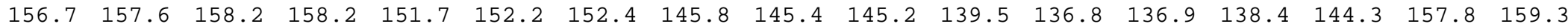

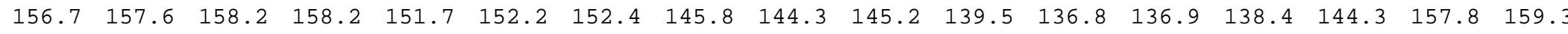

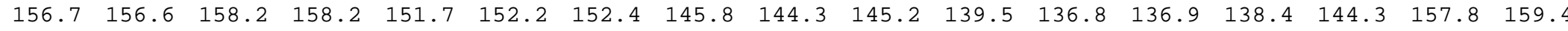

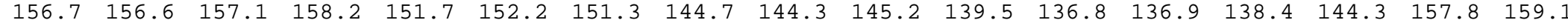

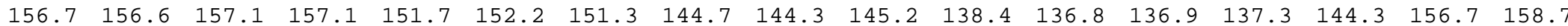

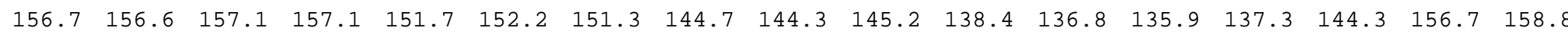

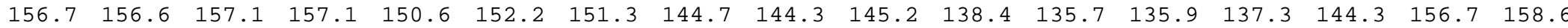

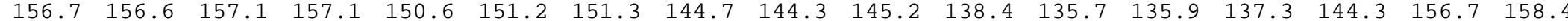

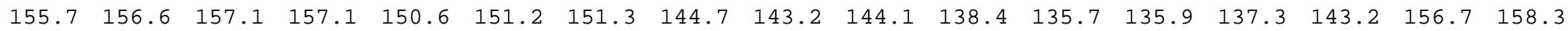




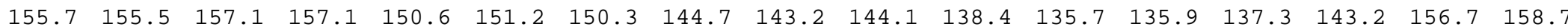

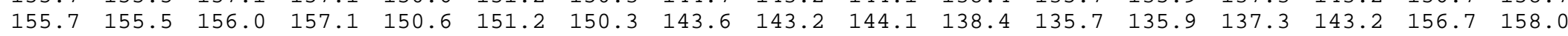

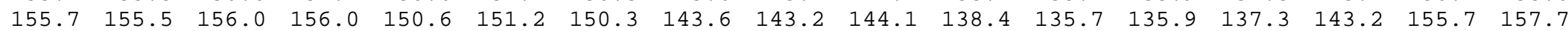

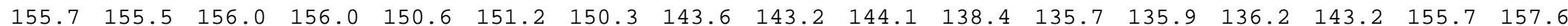

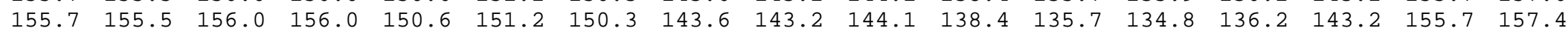

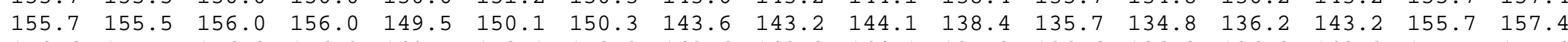

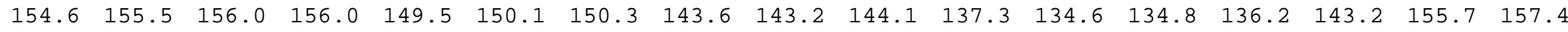

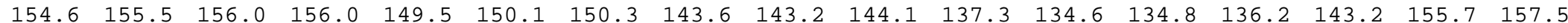

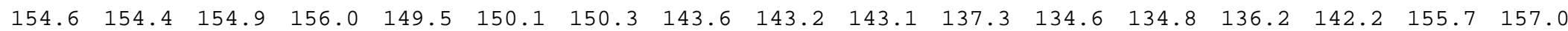

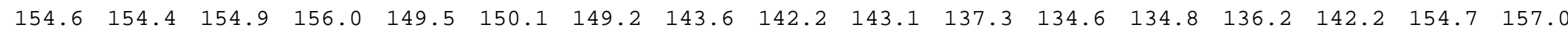

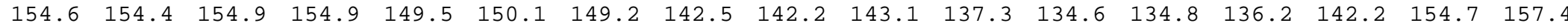

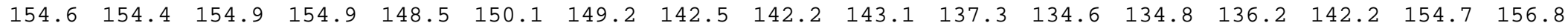

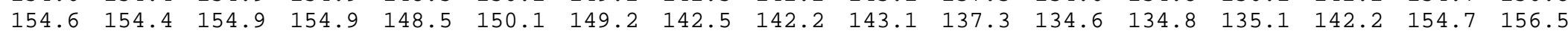

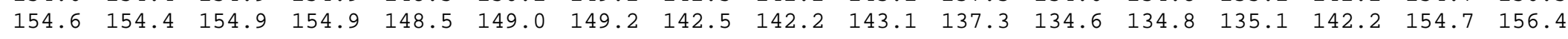

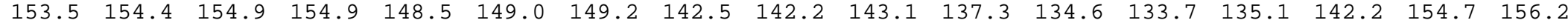

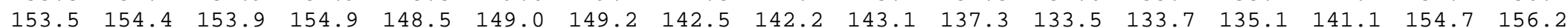

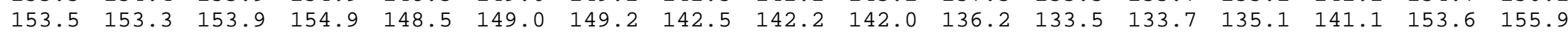

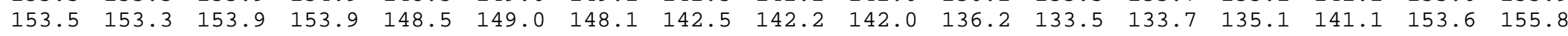

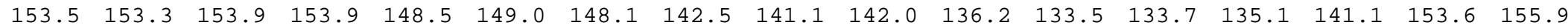

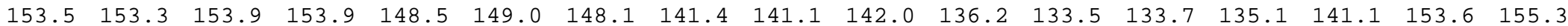

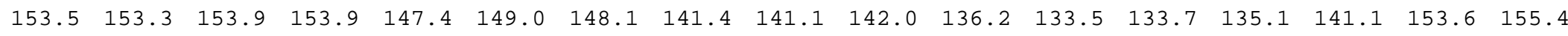

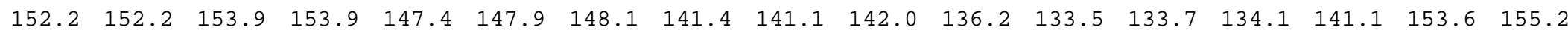

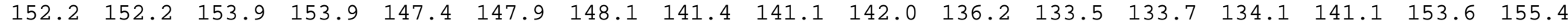

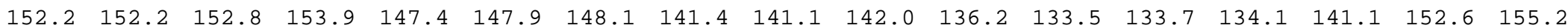

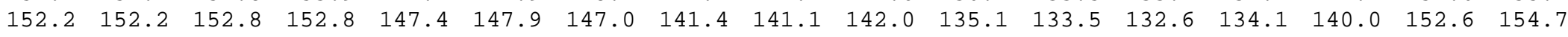

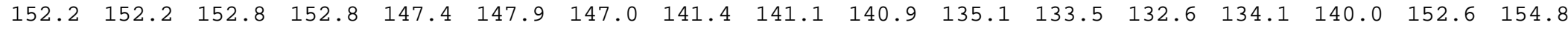

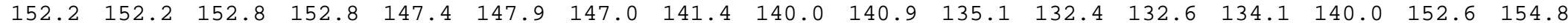

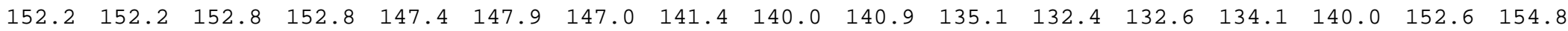

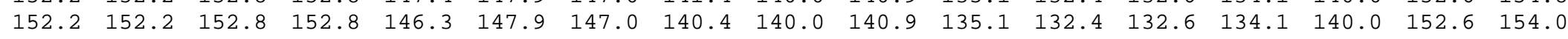

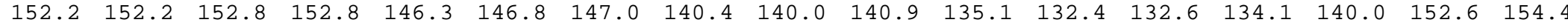

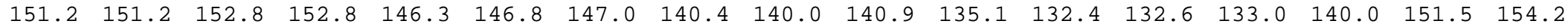

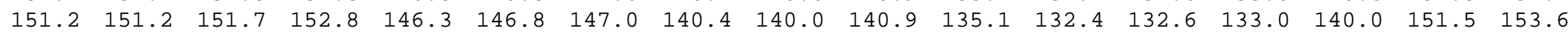

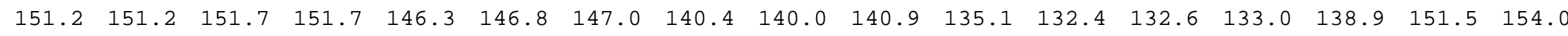

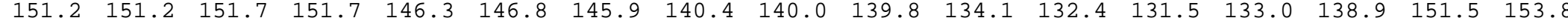

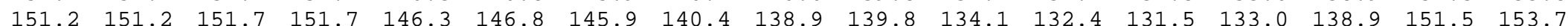

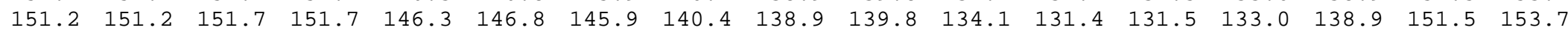

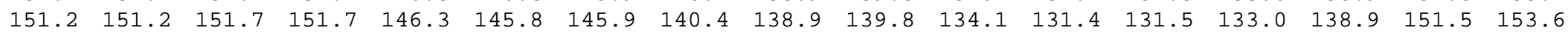

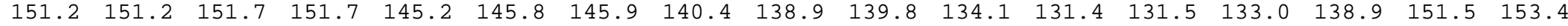

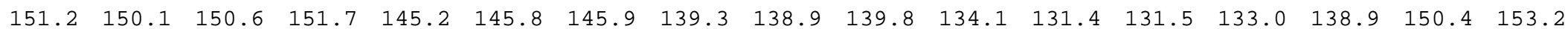

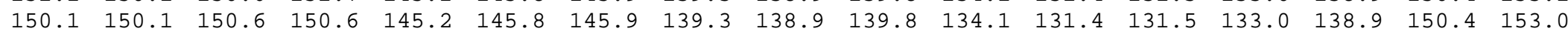

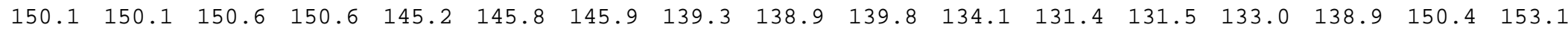

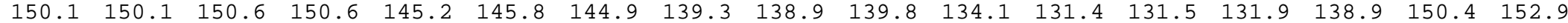

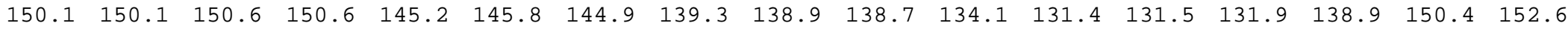

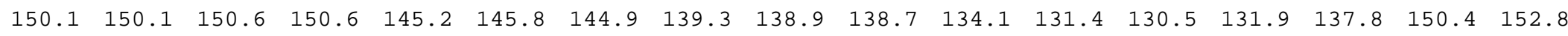

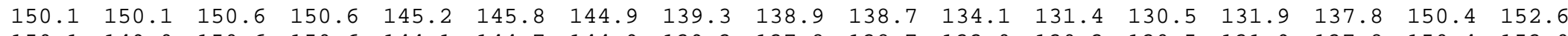

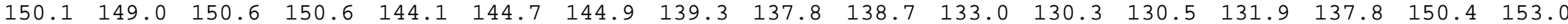

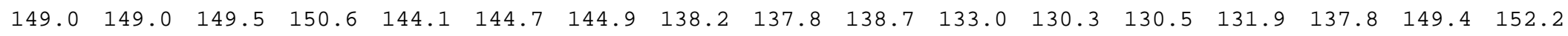




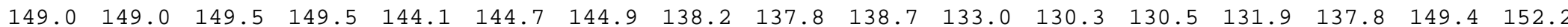

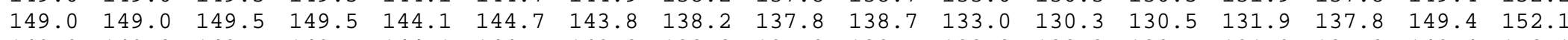

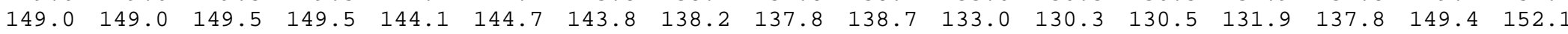

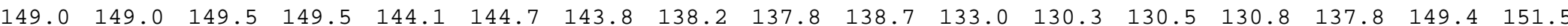

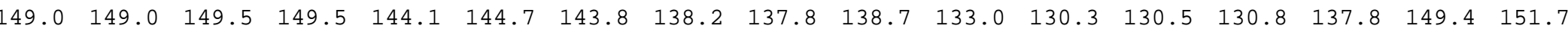

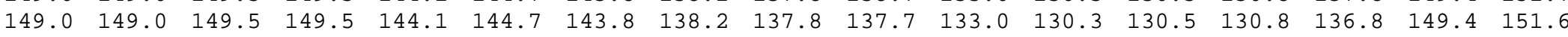

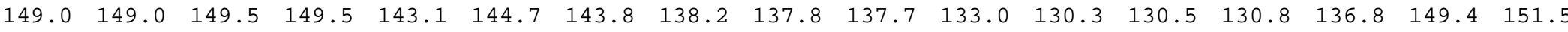

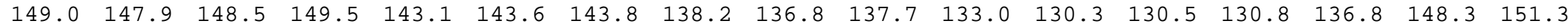

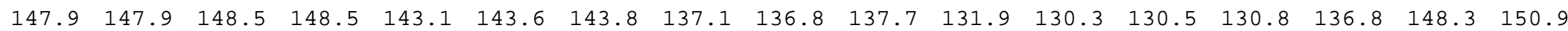

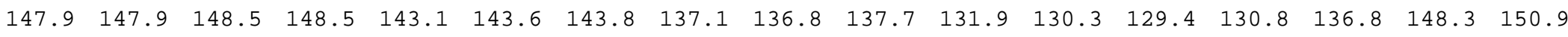

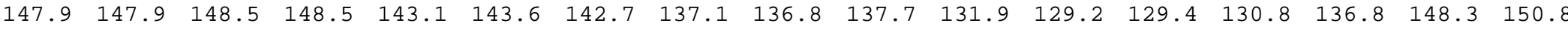

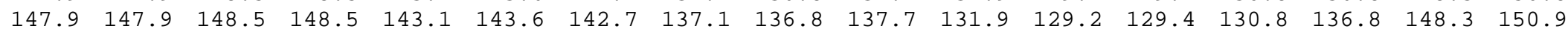

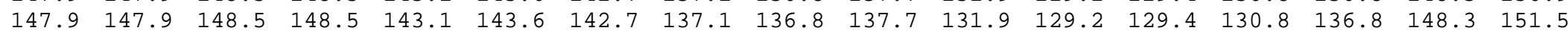

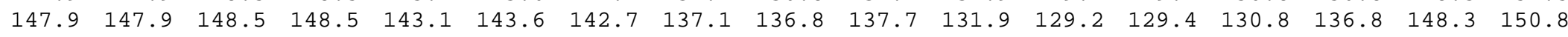

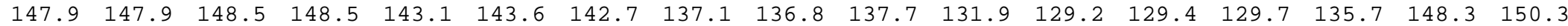

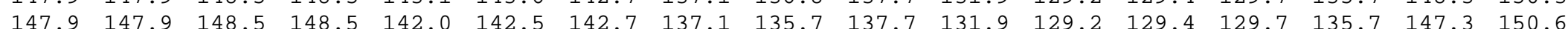

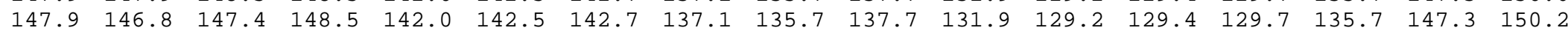

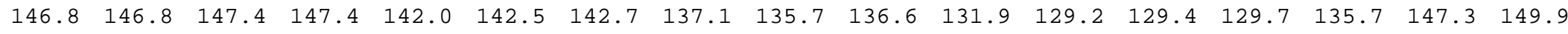

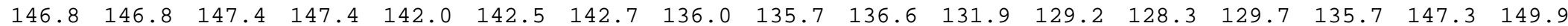

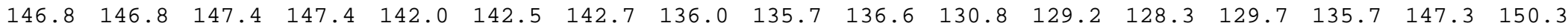

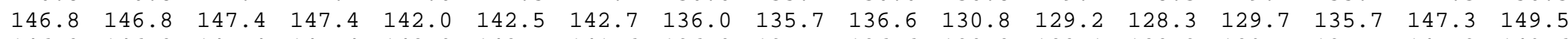

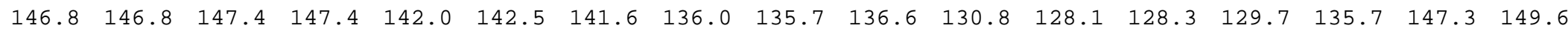

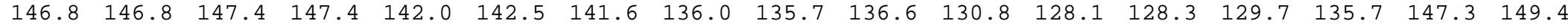

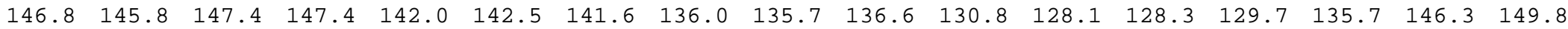

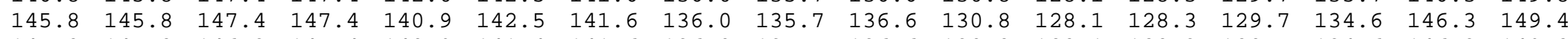

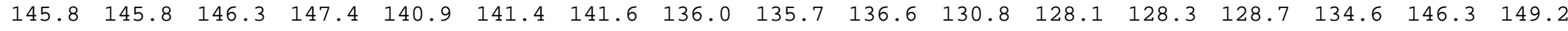

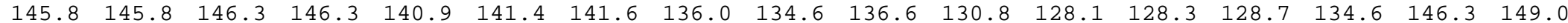

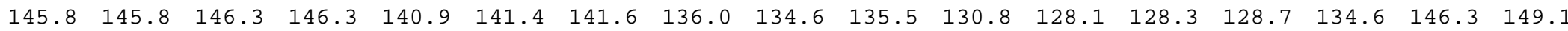

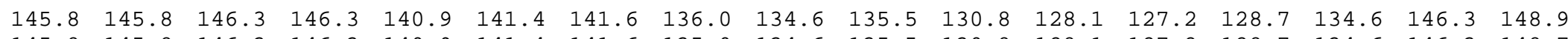

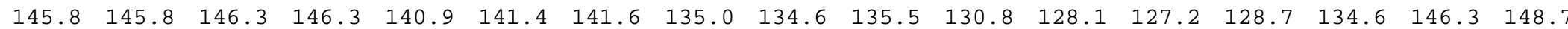

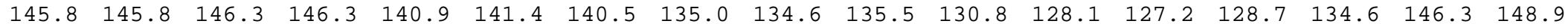

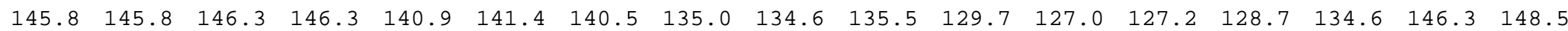

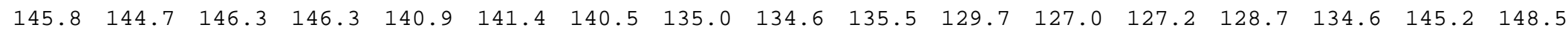

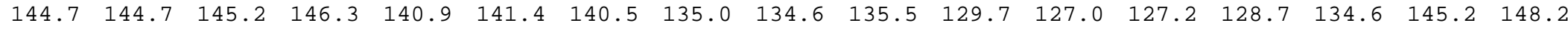

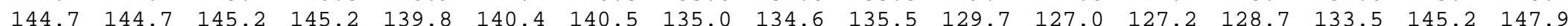

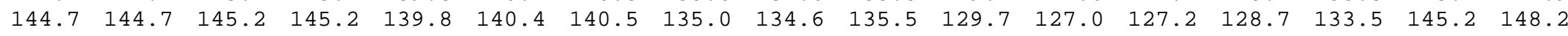

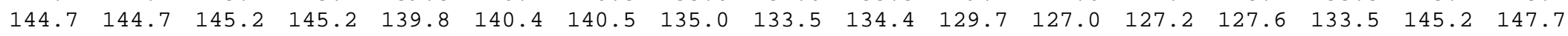

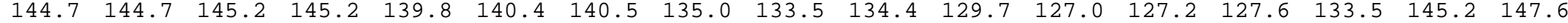

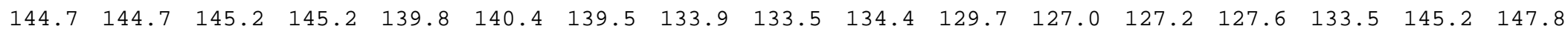

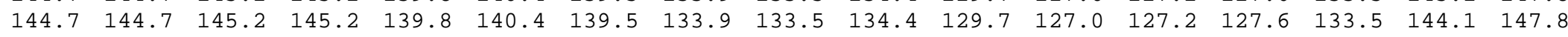

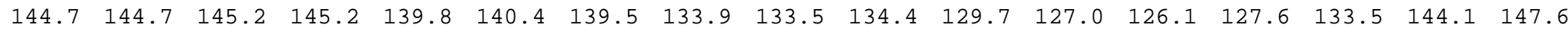

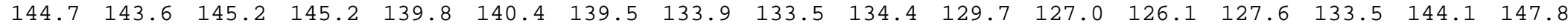

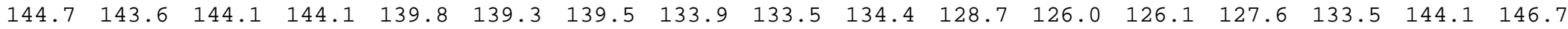

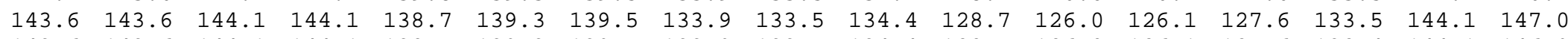

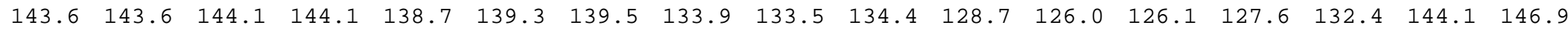

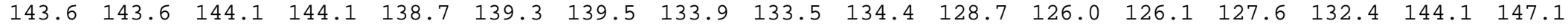

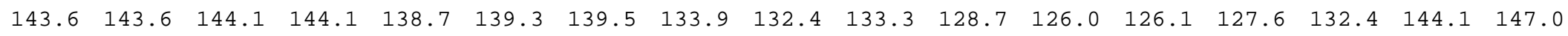


$\begin{array}{llllllllllllllllllll}143.6 & 143.6 & 144.1 & 144.1 & 138.7 & 139.3 & 138.4 & 133.9 & 132.4 & 133.3 & 128.7 & 126.0 & 126.1 & 126.5 & 132.4 & 144.1 & 147.0\end{array}$

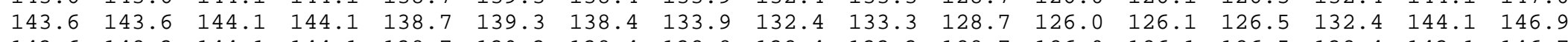

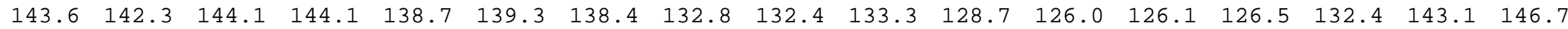

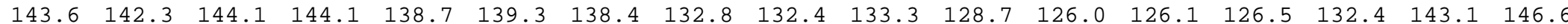

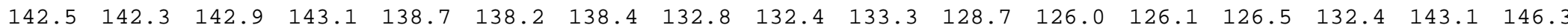

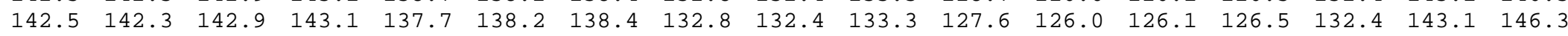

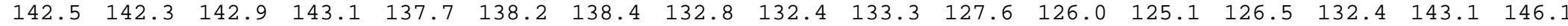

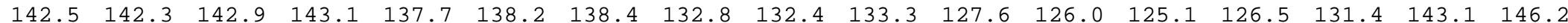

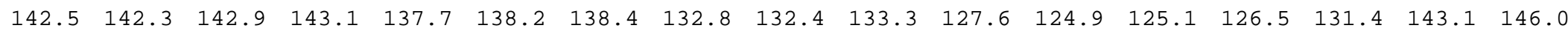

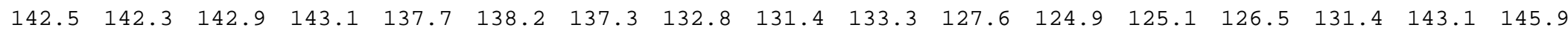

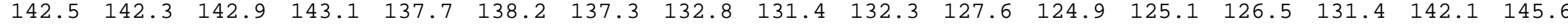

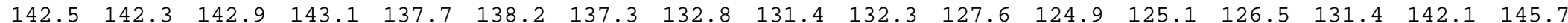

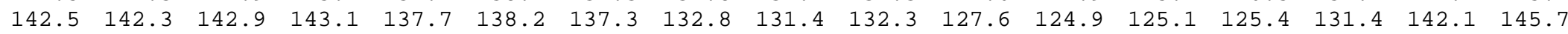

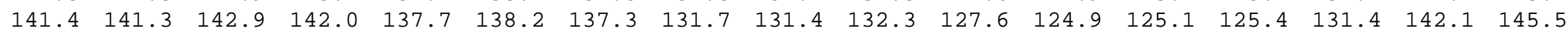

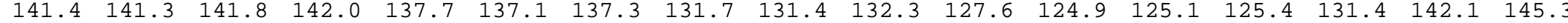

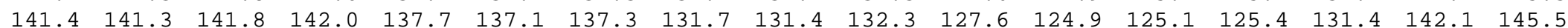

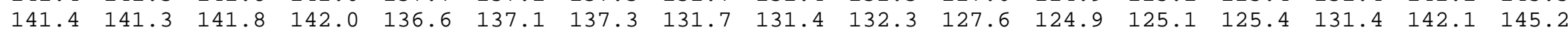

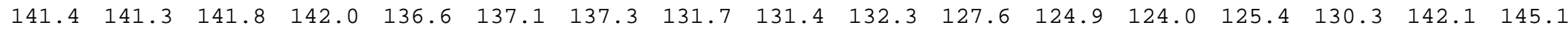

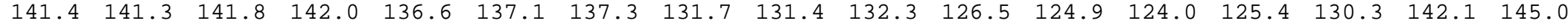

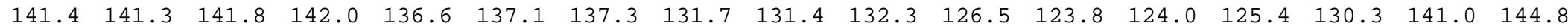

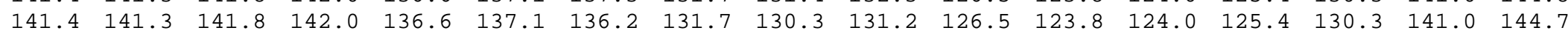

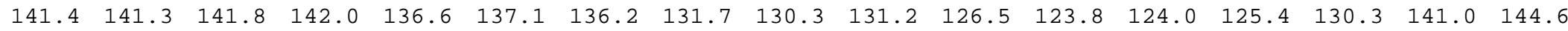

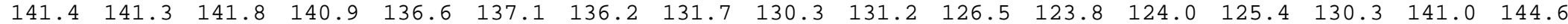

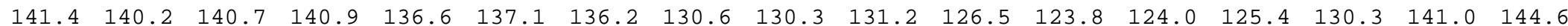

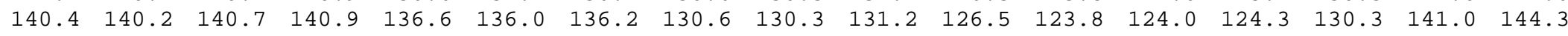

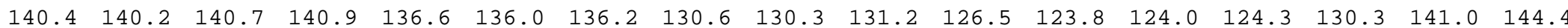

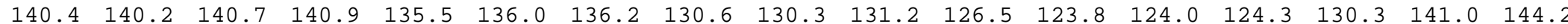

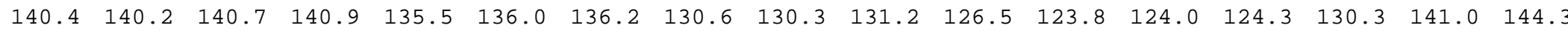

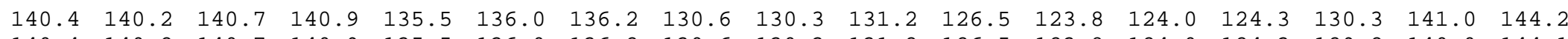

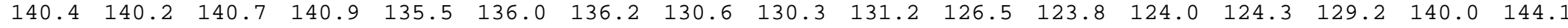

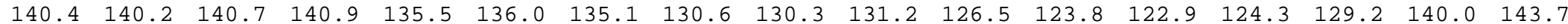

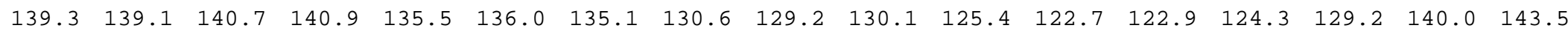

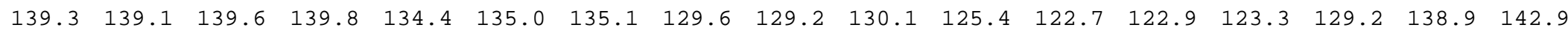

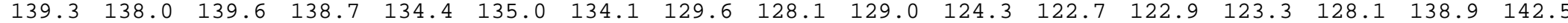

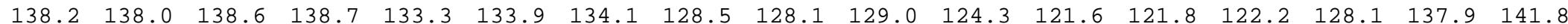

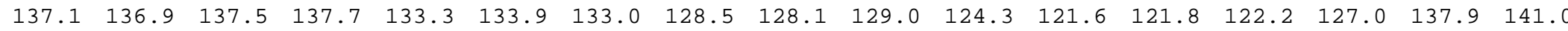

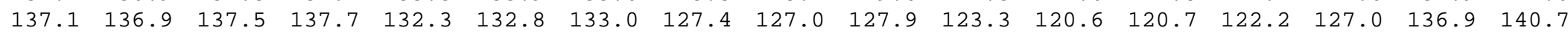

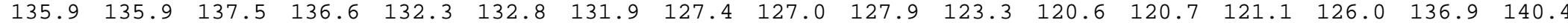

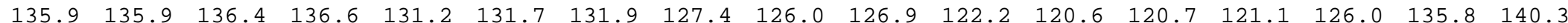

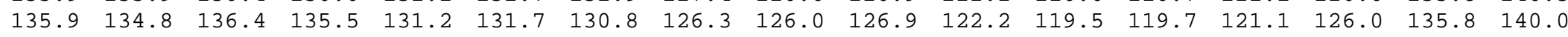

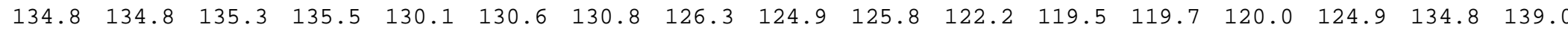

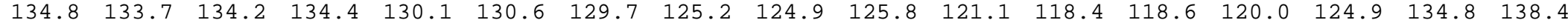

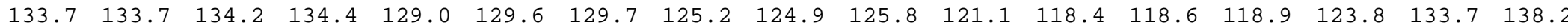

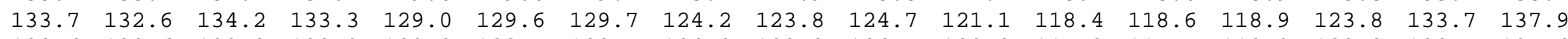

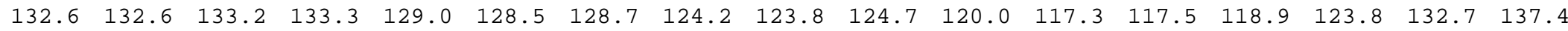

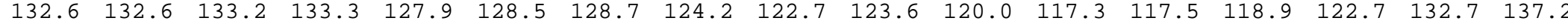

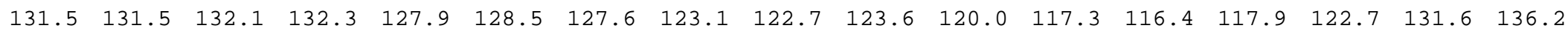




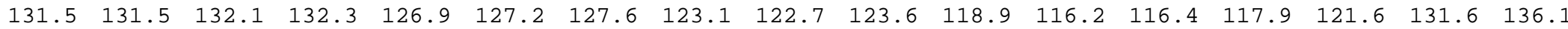

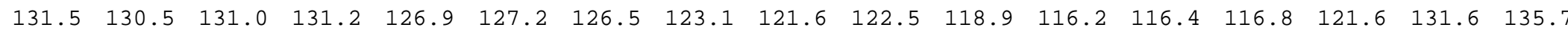

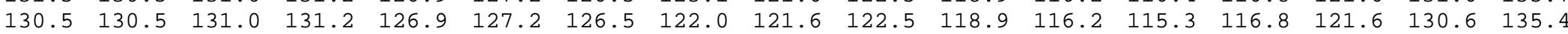

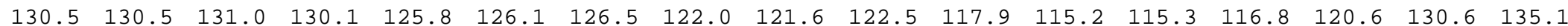

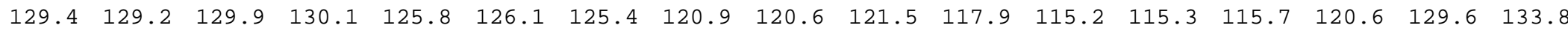

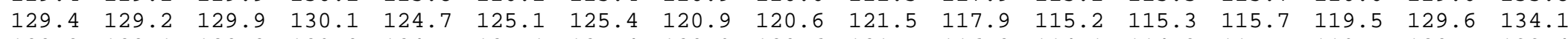

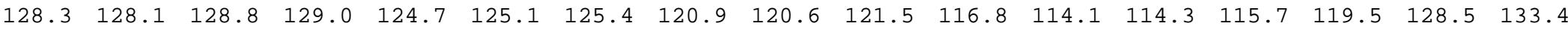

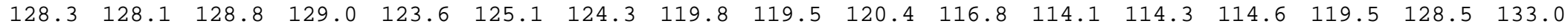

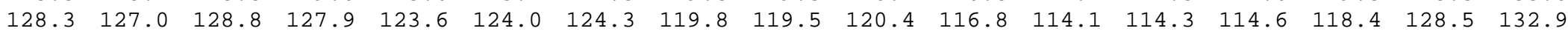

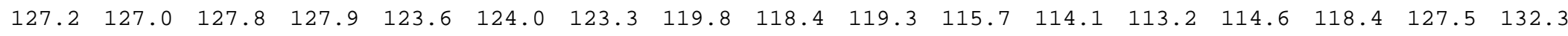

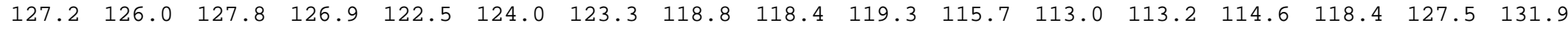

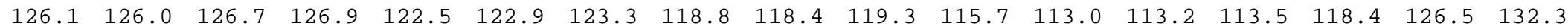

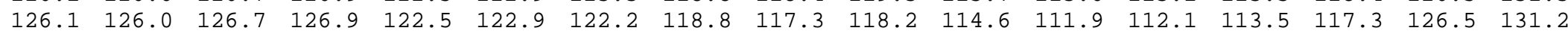

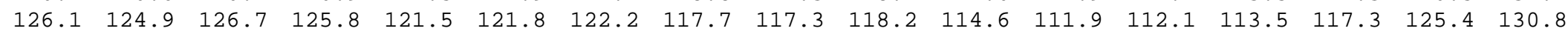

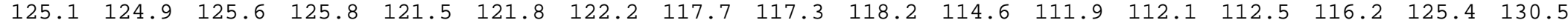

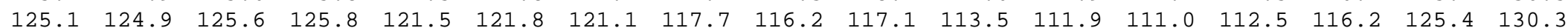

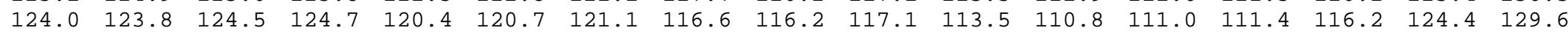

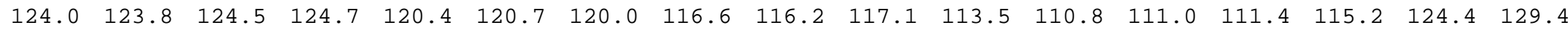

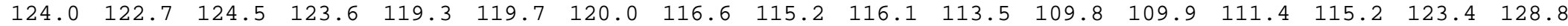

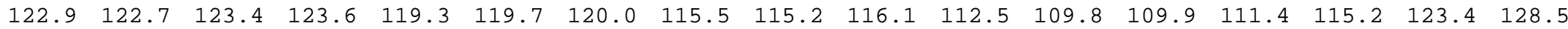

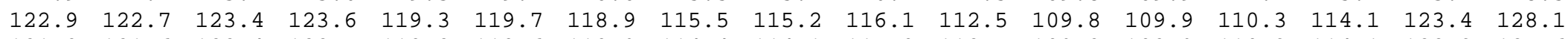

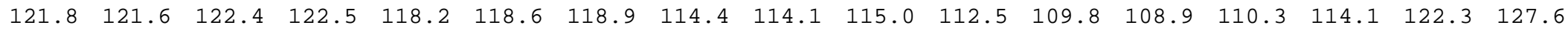

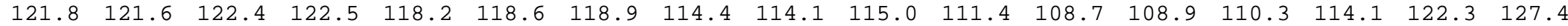

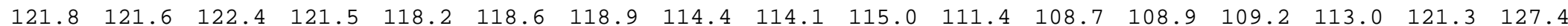

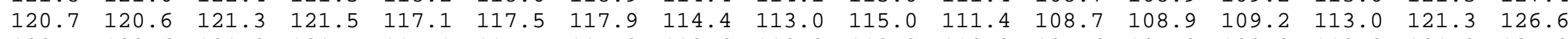

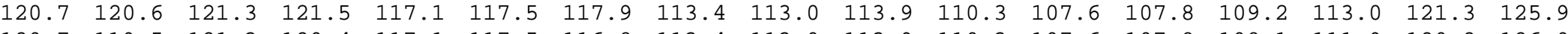

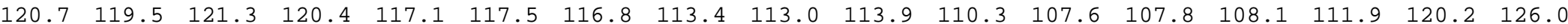

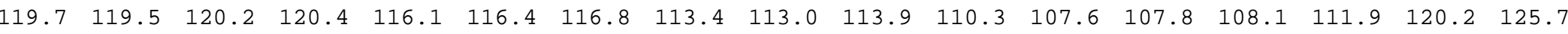

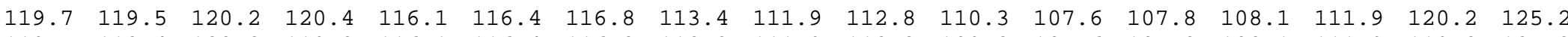

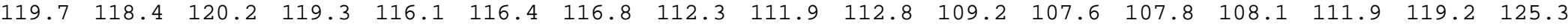

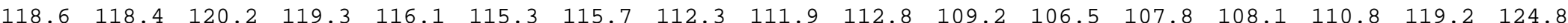

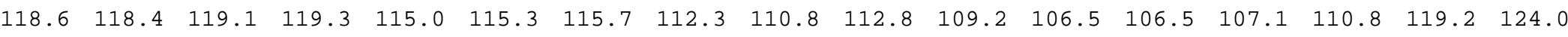

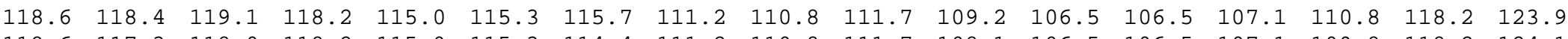

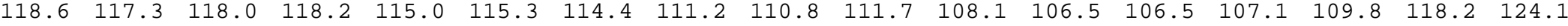

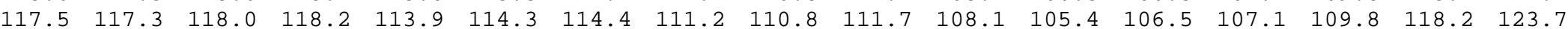

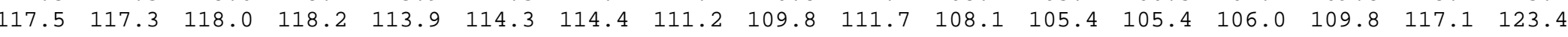

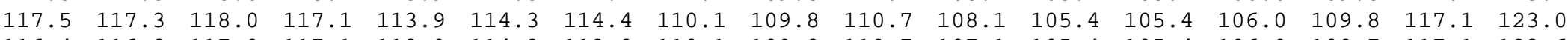

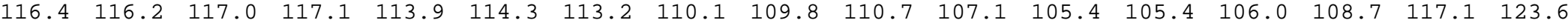

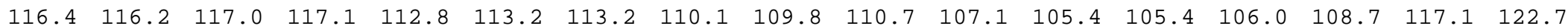

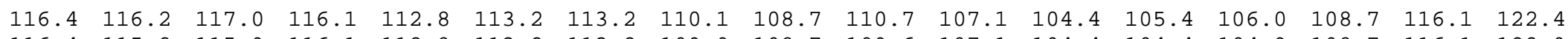

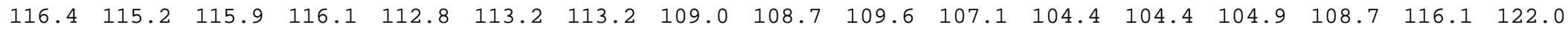

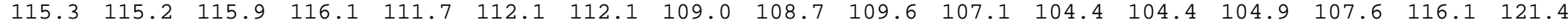

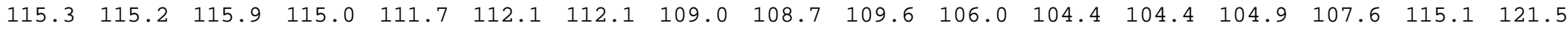

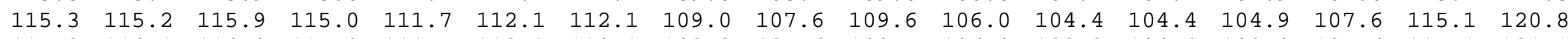

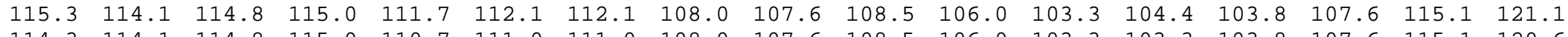

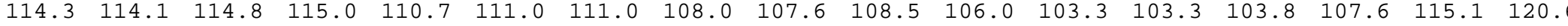

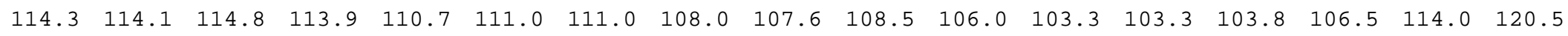




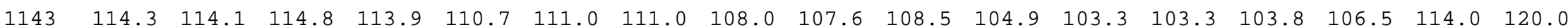

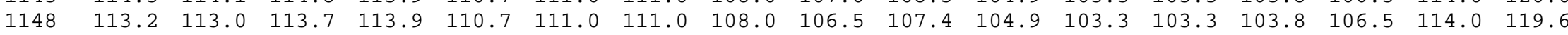

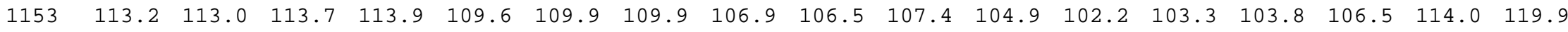

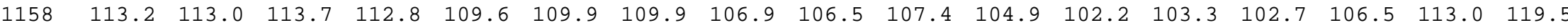

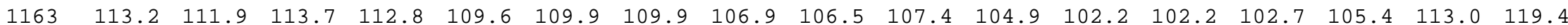

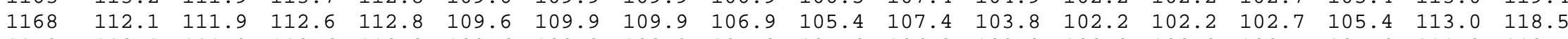

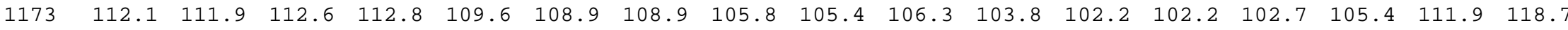

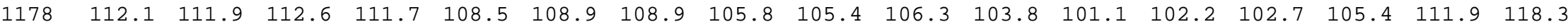

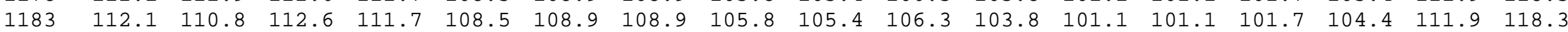

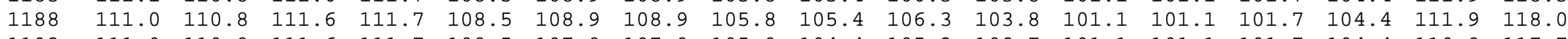

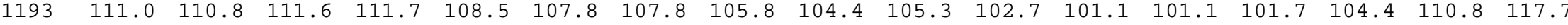

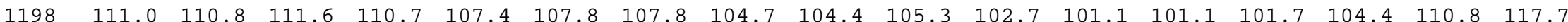

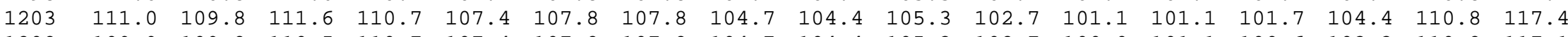

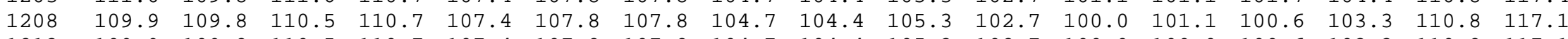

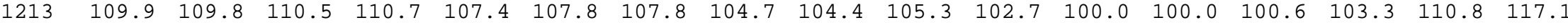

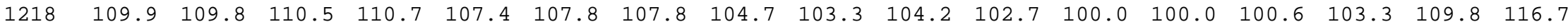

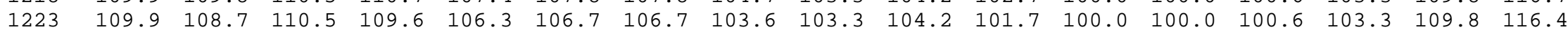

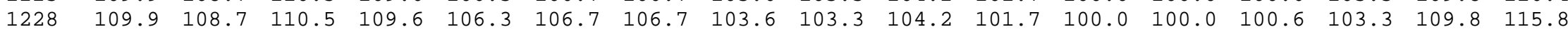

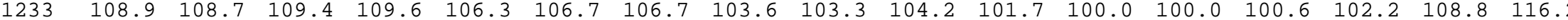

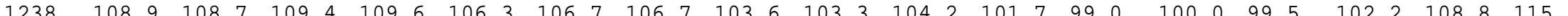

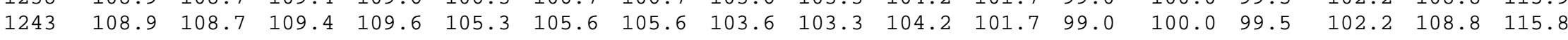

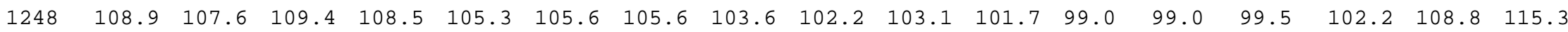

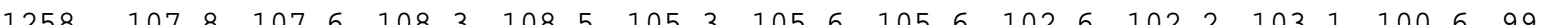

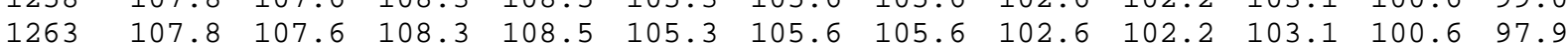

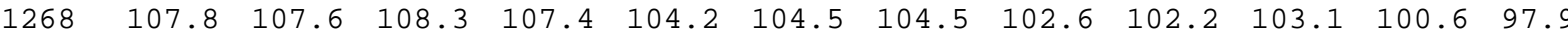

$\begin{array}{lllllllllllll}1273 & 107.8 & 107.6 & 108.3 & 107.4 & 104.2 & 104.5 & 104.5 & 102.6 & 101.1 & 102.0 & 100.6 & 97.9\end{array}$

$\begin{array}{lllllllllllll}1278 & 106.7 & 106.3 & 107.2 & 107.4 & 104.2 & 104.5 & 104.5 & 101.5 & 101.1 & 102.0 & 100.6 & 97.9\end{array}$

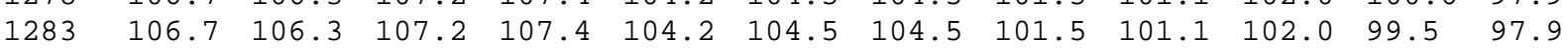

$\begin{array}{lllllllllllll}1288 & 106.7 & 106.3 & 107.2 & 107.4 & 104.2 & 104.5 & 104.5 & 101.5 & 101.1 & 102.0 & 99.5 & 97.9\end{array}$

$\begin{array}{lllllllllllll}1293 & 106.7 & 106.3 & 107.2 & 106.3 & 104.2 & 103.5 & 103.5 & 101.5 & 101.1 & 102.0 & 99.5 & 97.9\end{array}$

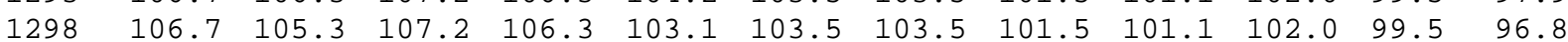

$\begin{array}{lllllllllllll}1303 & 105.6 & 105.3 & 106.2 & 106.3 & 103.1 & 103.5 & 103.5 & 100.4 & 100.0 & 100.9 & 99.5 & 96.8\end{array}$

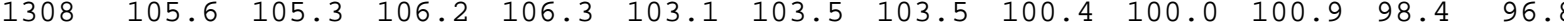

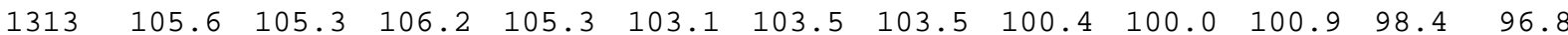

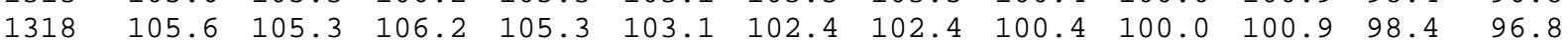

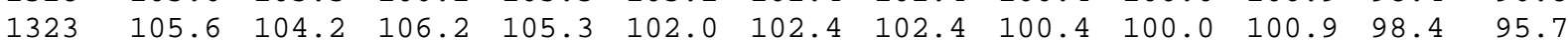

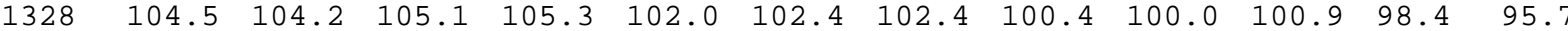

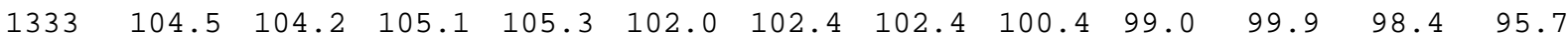

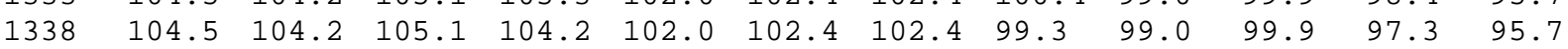

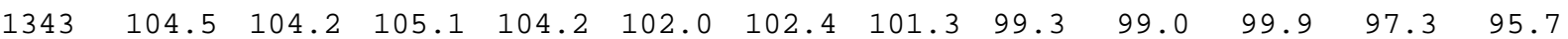

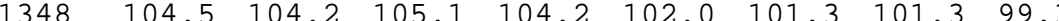

$\begin{array}{lllllllll}1353 & 104.5 & 104.2 & 104.0 & 104.2 & 100.9 & 101.3 & 101.3 & 99.3\end{array}$

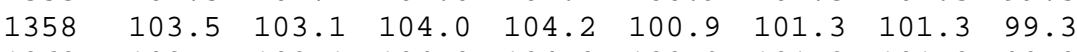

$\begin{array}{lllllllll}1363 & 103.5 & 103.1 & 104.0 & 104.2 & 100.9 & 101.3 & 101.3 & 99.3\end{array}$

$\begin{array}{lllllllll}1368 & 103.5 & 103.1 & 104.0 & 103.1 & 100.9 & 101.3 & 101.3 & 98.2\end{array}$

$\begin{array}{llll}99.0 & 99.9 & 97.3 & 95.7\end{array}$

$99.0 \quad 99.9 \quad 97.3 \quad 95.7$

$99.0 \quad 99.9 \quad 97.3 \quad 95.7$

$97.9 \quad 98.8 \quad 97.3 \quad 94.6$

1373

103.5 103.1 104.0 103.1100 .9 101

$97.9 \quad 98.8 \quad 97.3 \quad 94.6$

$\begin{array}{lllll}99.0 & 99.5 & 102.2 & 107.7 & 115.1\end{array}$

$\begin{array}{ccccc}99.0 & 99.5 & 101.1 & 107.7 & 115.1\end{array}$

$\begin{array}{lllll}99.0 & 98.4 & 101.1 & 107.7 & 114.4\end{array}$

$97.9 \quad 98.4 \quad 101.1 \quad 107.7 \quad 114.5$

$\begin{array}{lllll}97.9 & 98.4 & 101.1 & 107.7 & 114.1\end{array}$

$\begin{array}{lllll}97.9 & 98.4 & 101.1 & 106.7 & 114.1\end{array}$

$\begin{array}{lllll}97.9 & 98.4 & 101.1 & 106.7 & 114.1\end{array}$

$\begin{array}{lllll}97.9 & 98.4 & 100.0 & 106.7 & 113.1\end{array}$

$\begin{array}{lllll}97.9 & 97.3 & 100.0 & 106.7 & 113.3\end{array}$

$\begin{array}{lllll}96.8 & 97.3 & 100.0 & 106.7 & 113.2\end{array}$

$\begin{array}{lllll}96.8 & 97.3 & 100.0 & 105.6 & 113.2\end{array}$

$\begin{array}{lllll}96.8 & 97.3 & 100.0 & 105.6 & 112.6\end{array}$

$\begin{array}{lllll}96.8 & 97.3 & 99.0 & 105.6 & 112.5\end{array}$

$\begin{array}{lllll}96.8 & 97.3 & 99.0 & 105.6 & 112.5\end{array}$

$\begin{array}{lllll}96.8 & 96.3 & 99.0 & 105.6 & 112.4\end{array}$

$\begin{array}{lllll}95.7 & 96.3 & 99.0 & 104.5 & 112.0\end{array}$

$\begin{array}{lllll}95.7 & 96.3 & 99.0 & 104.5 & 111.2\end{array}$

$\begin{array}{lllll}95.7 & 96.3 & 99.0 & 104.5 & 111.8\end{array}$

$\begin{array}{lllll}95.7 & 96.3 & 97.9 & 104.5 & 111.7\end{array}$

$\begin{array}{lllll}95.7 & 96.3 & 97.9 & 104.5 & 111.5\end{array}$

$\begin{array}{lllll}95.7 & 96.3 & 97.9 & 103.5 & 111.3\end{array}$

$\begin{array}{lllll}95.7 & 96.3 & 97.9 & 103.5 & 111.0\end{array}$ 


\begin{tabular}{|c|c|c|c|c|c|c|c|c|c|c|c|c|c|c|c|c|c|}
\hline 1378 & 103.5 & 103.1 & 104.0 & 103.1 & 100.9 & 100.2 & 100.2 & 98.2 & 97.9 & 98.8 & 96.3 & 94.6 & 94.6 & 95.2 & 96.8 & 103.5 & 110.6 \\
\hline 1383 & 102.4 & 102.0 & 102.9 & 103.1 & 100.9 & 100.2 & 100.2 & 98.2 & 97.9 & 98.8 & 96.3 & 94.6 & 94.6 & 95.2 & 96.8 & 103.5 & 110.3 \\
\hline 1388 & 102.4 & 102.0 & 102.9 & 103.1 & 99.9 & 100.2 & 100.2 & 98.2 & 97.9 & 98.8 & 96.3 & 94.6 & 94.6 & 95.2 & 96.8 & 102.5 & 109.8 \\
\hline 393 & 102.4 & 102.0 & 102.9 & 103.1 & 99.9 & 100.2 & 100.2 & 98.2 & 97.9 & 98.8 & 96.3 & 94.6 & 94.6 & 95.2 & 96.8 & 102.5 & 109.8 \\
\hline 398 & 102.4 & 102.0 & 102.9 & 103.1 & 99.9 & 100.2 & 100.2 & 98.2 & 97.9 & 98.8 & 96.3 & 94.6 & 94.6 & 95.2 & 96.8 & 102.5 & 110.1 \\
\hline 403 & 102.4 & 102.0 & 102.9 & 102.0 & 99.9 & 100.2 & 100.2 & 98.2 & 96.8 & 97.7 & 96.3 & 94.6 & 94.6 & 95.2 & 96.8 & 102.5 & 109.8 \\
\hline 408 & 102.4 & 102.0 & 102.9 & 102.0 & 99.9 & 100.2 & 100.2 & 97.2 & 96.8 & 97.7 & 96.3 & 94.6 & 94.6 & 95.2 & 96.8 & 102.5 & 109.9 \\
\hline 413 & 102.4 & 102.0 & 102.9 & 102.0 & 99.9 & 100.2 & 100.2 & 97.2 & 96.8 & 97.7 & 96.3 & 94.6 & 94.6 & 95.2 & 96.8 & 102.5 & 109.8 \\
\hline 418 & 102.4 & 100.9 & 101.8 & 102.0 & 99.9 & 100.2 & 100.2 & 97.2 & 96.8 & 97.7 & 96.3 & 93.6 & 94.6 & 95.2 & 96.8 & 102.5 & 109.6 \\
\hline 423 & 101.3 & 100.9 & 101.8 & 102.0 & 99.9 & 99.1 & 99.1 & 97.2 & 96.8 & 97.7 & 96.3 & 93.6 & 93.6 & 94.1 & 95.7 & 101.4 & 109.4 \\
\hline 428 & 101.3 & 100.9 & 101.8 & 102.0 & 99.9 & 99.1 & 99.1 & 97.2 & 96.8 & 97.7 & 96.3 & 93.6 & 93.6 & 94.1 & 95.7 & 101.4 & 109.2 \\
\hline 433 & 101.3 & 100.9 & 101.8 & 102.0 & 98.8 & 99.1 & 99.1 & 97.2 & 96.8 & 97.7 & 96.3 & 93.6 & 93.6 & 94.1 & 95.7 & 101.4 & 109.2 \\
\hline 438 & 101.3 & 100.9 & 101.8 & 102.0 & 98.8 & 99.1 & 99.1 & 97.2 & 96.8 & 97.7 & 95.2 & 93.6 & 93.6 & 94.1 & & 101.4 & 109.0 \\
\hline 443 & 101.3 & 100.9 & 101.8 & 100.9 & 98.8 & 99.1 & 99.1 & 97.2 & 96.8 & & 95.2 & & 93.6 & 94.1 & & 101.4 & 109.2 \\
\hline 448 & 101.3 & 100.9 & 101.8 & 100.9 & 98.8 & 99.1 & 99.1 & 97.2 & & & 95.2 & & & 94.1 & & 101.4 & 108.8 \\
\hline 453 & 01.3 & 100.9 & 101.8 & 100.9 & 98.8 & 99.1 & 99.1 & 97.2 & & & 95.2 & & & 94.1 & & 101.4 & 9.0 \\
\hline 458 & 101.3 & 100.9 & 100.8 & 100.9 & 98.8 & 99.1 & 99.1 & 97.2 & & & 95.2 & & & 94.1 & & 1.4 & 108.9 \\
\hline 463 & 100.2 & 99.9 & 100.8 & 100.9 & 98.8 & 99.1 & 99.1 & 96.1 & & & 95.2 & & & & & 0.4 & \\
\hline 468 & 100.2 & 99.9 & 100.8 & 100.9 & 98.8 & 98.1 & 98.1 & 96.1 & & & 95.2 & & 93.6 & 94.1 & & .4 & \\
\hline 473 & 100.2 & 99.9 & 100.8 & 100.9 & 98.8 & 98.1 & 98.1 & 96.1 & 95 & 96.6 & 95.2 & & 93.6 & 94.1 & & 100.4 & 108.7 \\
\hline 478 & 00.2 & 99.9 & 100.8 & 100.9 & 97.7 & 98.1 & 98.1 & 96.1 & 95.7 & 96.6 & 95.2 & 93.6 & 93.6 & 93.0 & 94.6 & 100.4 & 108.4 \\
\hline 483 & $0 \odot .2$ & 99.9 & 100.8 & 99.9 & 97.7 & 98.1 & 98.1 & 96.1 & 95.7 & 96.6 & 95.2 & 93.6 & 93.6 & 93.0 & 94.6 & 100.4 & 108.3 \\
\hline 488 & $\odot \odot .2$ & 9.9 & 100.8 & 9.9 & 97.7 & 98.1 & 98.1 & 96.1 & 95 & 96.6 & 95.2 & & 93.6 & 93.0 & 94.6 & 100.4 & 108.9 \\
\hline 493 & $\odot \odot .2$ & 99.9 & 100.8 & 9.9 & 97.7 & 98.1 & 98.1 & 96.1 & 95 & 96. & 95.2 & .5 & 92.5 & 93.0 & 94.6 & 100.4 & 108.3 \\
\hline 498 & 100.2 & 99.9 & 99.7 & 99.9 & 97.7 & 98.1 & 98.1 & 96.1 & 95.7 & 96.6 & 95.2 & 92.5 & 92.5 & 93.0 & 94.6 & 100.4 & 108.1 \\
\hline 503 & 100.2 & 99.9 & 99.7 & 9.9 & 97.7 & 98.1 & 98.1 & 96.1 & 95.7 & 95.5 & 94.1 & 92.5 & 92.5 & 93.0 & 94.6 & 100.4 & 107.9 \\
\hline 508 & 99.1 & 98.8 & 99.7 & 9.9 & 97.7 & 98.1 & 98.1 & 96.1 & 95.7 & 95.5 & 94.1 & 92.5 & 92.5 & $93 . \odot$ & 94.6 & 9.3 & 107.6 \\
\hline 513 & 9.1 & 98.8 & 99.7 & 9.9 & 97.7 & 97.0 & 97.0 & 95.0 & 94.6 & 95. & 94.1 & .5 & 92.5 & $93 . \odot$ & 94.6 & 99.3 & 107.5 \\
\hline 518 & 9.1 & 98.8 & 99.7 & 9.9 & 96.6 & 97.0 & 97.0 & 95.0 & 94.6 & 95.5 & 94.1 & .5 & 92.5 & $93 . \odot$ & 94.6 & 9.3 & 107.4 \\
\hline 523 & 9.1 & 98.8 & 99.7 & 9.9 & 96.6 & 97.0 & 97.0 & $95 . \odot$ & 94 & 95 & 94.1 & & 92.5 & 93.0 & 94.6 & 9.3 & 107.5 \\
\hline 528 & 9.1 & 98.8 & 99.7 & 9.9 & 96.6 & $97 . \odot$ & 97.0 & $95 . \odot$ & 94 & 95 & 94.1 & & 92 & .9 & .6 & 9.3 & 107.2 \\
\hline 533 & 9.1 & 98.8 & 99.7 & 98.8 & 96.6 & 97.0 & 97.0 & $95 . \odot$ & 94.6 & 95. & 94.1 & .5 & 92.5 & 91.9 & 93.6 & 99.3 & 107.3 \\
\hline 538 & 9.1 & 98.8 & 99.7 & 98.8 & 96.6 & $97 . \odot$ & 97.0 & 95.0 & 94 & 95.5 & 94.1 & .4 & 92.5 & 91.9 & 93.6 & 99.3 & 107.2 \\
\hline 543 & 9.1 & 98.8 & 98.6 & 98.8 & 96.6 & 97.0 & 97.0 & 95.0 & 94.6 & 95.5 & 94.1 & 91.4 & 92.5 & 91.9 & 93.6 & 99.3 & 106.5 \\
\hline 548 & 98.1 & 98.8 & 98.6 & 98.8 & 96.6 & 97.0 & 97.0 & 95.0 & 94.6 & 95.5 & 94.1 & 91.4 & 92.5 & 91.9 & 93.6 & 98.2 & 107.0 \\
\hline 553 & 98.1 & 97.7 & 98.6 & 98.8 & 96.6 & 95.9 & 95.9 & 95.0 & 94.6 & 94.5 & 93.0 & 91.4 & 91.4 & 91.9 & 93.6 & 98.2 & 106.3 \\
\hline 558 & 98.1 & 97.7 & 98.6 & 98.8 & 95.5 & 95.9 & 95.9 & 93.9 & 93.6 & 94.5 & 93.0 & 91.4 & 91.4 & 91.9 & 93.6 & 98.2 & 107.1 \\
\hline 563 & 98.1 & 97.7 & 98.6 & 98.8 & 95.5 & 95.9 & 95.9 & 93.9 & 93.6 & 94.5 & 93.0 & 91.4 & 91.4 & 91.9 & 93.6 & 98.2 & 105.7 \\
\hline 568 & 98.1 & 97.7 & 98.6 & 98.8 & 95.5 & 95.9 & 95.9 & 93.9 & 93.6 & 94.5 & 93.0 & 91.4 & 91.4 & 91.9 & 93.6 & 98.2 & 106.2 \\
\hline 573 & 98.1 & 97.7 & 98.6 & 97.7 & 95.5 & 95.9 & 95.9 & 93.9 & 93.6 & 94.5 & 93.0 & 91.4 & 91.4 & 91.9 & 92.5 & 98.2 & 106.2 \\
\hline 578 & 98.1 & 97.7 & 98.6 & 97.7 & 95.5 & 95.9 & 95.9 & 93.9 & 93.6 & 94.5 & 93.0 & 91.4 & 91.4 & 91.9 & 92.5 & 98.2 & 106.1 \\
\hline 583 & 98.1 & 97.7 & 98.6 & 97.7 & 95.5 & 95.9 & 95.9 & 93.9 & 93.6 & 94.5 & 93.0 & 91.4 & 91.4 & 91.9 & 92.5 & 98.2 & 106.0 \\
\hline 588 & 98.1 & 97.7 & 97.5 & 97.7 & 95.5 & 95.9 & 95.9 & 93.9 & 93.6 & 94.5 & 93.0 & 91.4 & 91.4 & 91.9 & 92.5 & 97.2 & 106.2 \\
\hline 593 & 97.0 & 97.7 & 97.5 & 97.7 & 95.5 & 95.9 & 95.9 & 93.9 & 93.6 & 94.5 & 93.0 & 91.4 & 91.4 & 91.9 & 92.5 & 97.2 & 106.1 \\
\hline 598 & 97.0 & 96.6 & 97.5 & 97.7 & 95.5 & 95.9 & 95.9 & 93.9 & 93.6 & 94.5 & 93.0 & 90.3 & 91.4 & 90.9 & 92.5 & 97.2 & 105.6 \\
\hline 603 & 97.0 & 96.6 & 97.5 & 97.7 & 94.5 & 95.9 & 95.9 & 93.9 & 93.6 & 94.5 & 93.0 & 90.3 & 91.4 & 90.9 & 92.5 & 97.2 & 106.2 \\
\hline 608 & 97.0 & 96.6 & 97.5 & 97.7 & 94.5 & 94.8 & 94.8 & 93.9 & 93.6 & 94.5 & 93.0 & 90.3 & 91.4 & 90.9 & 92.5 & 97.2 & 105.7 \\
\hline
\end{tabular}




\begin{tabular}{|c|c|c|c|c|c|c|c|c|c|c|c|c|c|c|c|c|c|}
\hline 1613 & 97.0 & 96.6 & 97.5 & 96.6 & 94.5 & 94.8 & 94.8 & 92.8 & 93.6 & 93.4 & 91.9 & 90.3 & 90.3 & 90.9 & 92.5 & 97.2 & 105.2 \\
\hline 1618 & 97.0 & 96.6 & 97.5 & 96.6 & 94.5 & 94.8 & 94.8 & 92.8 & 92.5 & 93.4 & 91.9 & 90.3 & 90.3 & 90.9 & 92.5 & 97.2 & 105.3 \\
\hline 1623 & 97.0 & 96.6 & 97.5 & 96.6 & 94.5 & 94.8 & 94.8 & 92.8 & 92.5 & 93.4 & 91.9 & 90.3 & 90.3 & 90.9 & 92.5 & 97.2 & 105.3 \\
\hline 1628 & 97.0 & 96.6 & 97.5 & 96.6 & 94.5 & 94.8 & 94.8 & 92.8 & 92.5 & 93.4 & 91.9 & 90.3 & 90.3 & 90.9 & 92.5 & 97.2 & 105.3 \\
\hline 1633 & 97.0 & 96.6 & 96.4 & 96.6 & 94.5 & 94.8 & 94.8 & 92.8 & 92.5 & 93.4 & 91.9 & 90.3 & 90.3 & 90.9 & 92.5 & 96.1 & 105.2 \\
\hline L638 & 95.9 & 95.5 & 96.4 & 96.6 & 94.5 & 94.8 & 94.8 & 92.8 & 92.5 & 93.4 & 91.9 & 90.3 & 90.3 & 90.9 & 92.5 & 96.1 & 104.8 \\
\hline 1643 & 95.9 & 95.5 & 96.4 & 96.6 & 94.5 & 94.8 & 94.8 & 92.8 & 92.5 & 93.4 & 91.9 & 90.3 & 90.3 & 90.9 & 92.5 & 96.1 & 104.9 \\
\hline 1648 & 95.9 & 95.5 & 96.4 & 96.6 & 94.5 & 94.8 & 94.8 & 92.8 & 92.5 & 93.4 & 91.9 & 90.3 & 90.3 & 90.9 & 91.4 & 96.1 & 104.9 \\
\hline 1653 & 95.9 & 95.5 & 96.4 & 96.6 & 94.5 & 94.8 & 94.8 & 92.8 & 92.5 & 93.4 & 91.9 & 90.3 & 90.3 & 90.9 & 91.4 & 96.1 & 104.9 \\
\hline 1658 & 95.9 & 95.5 & 96.4 & 95.5 & 93.4 & 93.7 & 93.7 & 92.8 & 92.5 & 93.4 & 91.9 & 90.3 & 90.3 & 90.9 & 91.4 & 96.1 & 104.7 \\
\hline 1663 & 95.9 & 95.5 & 96.4 & 95.5 & 93.4 & 93.7 & 93.7 & 92.8 & 92.5 & 93.4 & 91.9 & 90.3 & 90.3 & 90.9 & 91.4 & 96.1 & 104.7 \\
\hline 1668 & 95.9 & 95.5 & 96.4 & 95.5 & 93.4 & 93.7 & 93.7 & 92.8 & 92.5 & 93.4 & 91.9 & 89.2 & 90.3 & 89.8 & 91.4 & 96.1 & 104.4 \\
\hline 1673 & 95.9 & 95.5 & 96.4 & 95.5 & 93.4 & 93.7 & 93.7 & 92.8 & 92.5 & 92.3 & 91.9 & 89.2 & 90.3 & 89.8 & 91.4 & 96.1 & 104.7 \\
\hline 1678 & 95.9 & 95.5 & 96.4 & 95.5 & 93.4 & 93.7 & 93.7 & 92.8 & 92.5 & 92.3 & 91.9 & 89.2 & 89.2 & 89.8 & 91.4 & 96.1 & 104.7 \\
\hline 1683 & 95.9 & 95.5 & 96.4 & 95.5 & 93.4 & 93.7 & 93.7 & 92.8 & 92.5 & 92.3 & 91.9 & 89.2 & 89.2 & 89.8 & 91.4 & 95.1 & 104.4 \\
\hline 1688 & 95.9 & 94.5 & 95.4 & 95.5 & 93.4 & 93.7 & 93.7 & 91.8 & 91.2 & 92.3 & $9 \odot .9$ & 89.2 & 89.2 & 89.8 & 91.4 & 95.1 & 104.0 \\
\hline 1693 & 94.8 & 94.5 & 95.4 & 95.5 & 93.4 & 93.7 & 93.7 & 91.8 & 91.2 & 92.3 & 90.9 & 89.2 & 89.2 & 89.8 & 91.4 & 95.1 & 104.0 \\
\hline 1698 & 94.8 & 94.5 & 95.4 & 95.5 & 93.4 & 93.7 & 93.7 & 91.8 & 91.2 & 92.3 & 90.9 & 89.2 & 89.2 & 89.8 & 90.3 & 95.1 & 103.4 \\
\hline 1703 & 94.8 & 94.5 & 95.4 & 95.5 & 93.4 & 93.7 & 93.7 & 91.8 & 91.2 & 92.3 & 90.9 & 89.2 & 89.2 & 89.8 & 90.3 & 95.1 & 103.9 \\
\hline 1708 & 94.8 & 94.5 & 95.4 & 94.5 & 93.4 & 93.7 & 93.7 & 91.8 & 91.2 & 92.3 & 90.9 & 89.2 & 89.2 & 89.8 & 90.3 & 95.1 & 103.8 \\
\hline 1713 & 94.8 & 94.5 & 95.4 & 94.5 & 92.3 & 92.7 & 92.7 & 91.8 & 91.2 & 92.3 & 90.9 & 89.2 & 89.2 & 89.8 & 90.3 & 95.1 & 103.6 \\
\hline 1718 & 94.8 & 94.5 & 95.4 & 94.5 & 92.3 & 92.7 & 92.7 & 91.8 & 91.2 & 92.3 & 90.9 & 89.2 & 89.2 & 89.8 & 90.3 & 95.1 & 103.5 \\
\hline 1723 & 94.8 & 94.5 & 95.4 & 94.5 & 92.3 & 92.7 & 92.7 & 91.8 & 91.2 & 92.3 & 90.9 & 89.2 & 89.2 & 89.8 & 90.3 & 95.1 & 103.3 \\
\hline 1728 & 94.8 & 94.5 & 95.4 & 94.5 & 92.3 & 92.7 & 92.7 & 91.8 & 91.2 & 92.3 & 90.9 & 88.2 & 89.2 & 88.7 & 90.3 & 94.1 & 103.4 \\
\hline 1733 & 94.8 & 94.5 & 94.3 & 94.5 & 92.3 & 92.7 & 92.7 & 91.8 & 91.2 & 91.2 & 90.9 & 88.2 & 88.2 & 88.7 & 90.3 & 94.1 & 103.0 \\
\hline 1738 & 94.8 & 93.4 & 94.3 & 94.5 & 92.3 & 92.7 & 92.7 & 90.7 & 91.2 & 91.2 & $9 \odot .9$ & 88.2 & 88.2 & 88.7 & 90.3 & 94.1 & 103.1 \\
\hline 1743 & 93.7 & 93.4 & 94.3 & 94.5 & 92.3 & 92.7 & 92.7 & 90.7 & 91.2 & 91.2 & $9 \odot .9$ & 88.2 & 88.2 & 88.7 & 90.3 & 94.1 & 103.0 \\
\hline 1748 & 93.7 & 93.4 & 94.3 & 94.5 & 92.3 & 92.7 & 92.7 & 90.7 & 90.1 & 91.2 & 89.8 & 88.2 & 88.2 & 88.7 & 90.3 & 94.1 & 102.6 \\
\hline 1753 & 93.7 & 93.4 & 94.3 & 94.5 & 92.3 & 92.7 & 92.7 & 90.7 & 90.1 & 91.2 & 89.8 & 88.2 & 88.2 & 88.7 & 90.3 & 94.1 & 102.7 \\
\hline 1758 & 93.7 & 93.4 & 94.3 & 93.4 & 92.3 & 92.7 & 92.7 & 90.7 & 90.1 & 91.2 & 89.8 & 88.2 & 88.2 & 88.7 & 89.2 & 94.1 & 102.7 \\
\hline 1763 & 93.7 & 93.4 & 94.3 & 93.4 & 92.3 & 91.6 & 91.6 & 90.7 & 90.1 & 91.2 & 89.8 & 88.2 & 88.2 & 88.7 & 89.2 & 94.1 & 103.3 \\
\hline 1768 & 93.7 & 93.4 & 94.3 & 93.4 & 92.3 & 91.6 & 91.6 & 90.7 & 90.1 & 91.2 & 89.8 & 88.2 & 88.2 & 88.7 & 89.2 & 94.1 & 102.4 \\
\hline 1773 & 93.7 & 93.4 & 94.3 & 93.4 & 91.2 & 91.6 & 91.6 & 90.7 & 90.1 & 91.2 & 89.8 & 88.2 & 88.2 & 88.7 & 89.2 & 94.1 & 102.2 \\
\hline 1778 & 93.7 & 93.4 & 94.3 & 93.4 & 91.2 & 91.6 & 91.6 & 90.7 & 90.1 & 91.2 & 89.8 & 88.2 & 88.2 & 88.7 & 89.2 & 93.0 & 102.1 \\
\hline 1783 & 93.7 & 93.4 & 94.3 & 93.4 & 91.2 & 91.6 & 91.6 & 90.7 & $9 \odot .1$ & 91.2 & 89.8 & 88.2 & 88.2 & 88.7 & 89.2 & $93 . \odot$ & 102.4 \\
\hline 1788 & 92.7 & 93.4 & 93.2 & 93.4 & 91.2 & 91.6 & 91.6 & 90.7 & 90.1 & 91.2 & 89.8 & 88.2 & 88.2 & 88.7 & 89.2 & 93.0 & 102.1 \\
\hline 1793 & 92.7 & 92.3 & 93.2 & 93.4 & 91.2 & 91.6 & 91.6 & 90.7 & 90.1 & 91.2 & 89.8 & 88.2 & 88.2 & 88.7 & 89.2 & $93 . \odot$ & 102.2 \\
\hline 1798 & 92.7 & 92.3 & 93.2 & 93.4 & 91.2 & 91.6 & 91.6 & 90.7 & 90.1 & 90.1 & 89.8 & 88.2 & 88.2 & 87.6 & 89.2 & 93.0 & 101.8 \\
\hline 1803 & 92.7 & 92.3 & 93.2 & 93.4 & 91.2 & 91.6 & 91.6 & 89.6 & 90.1 & 90.1 & 89.8 & 87.1 & 88.2 & 87.6 & 89.2 & 93.0 & 101.7 \\
\hline 1808 & 92.7 & 92.3 & 93.2 & 92.3 & 91.2 & 91.6 & 91.6 & 89.6 & $9 \odot .1$ & 90.1 & 89.8 & 87.1 & 88.2 & 87.6 & 89.2 & $93 . \odot$ & 101.7 \\
\hline 1813 & 92.7 & 92.3 & 93.2 & 92.3 & 91.2 & 91.6 & 91.6 & 89.6 & 90.1 & 90.1 & 89.8 & 87.1 & 87.1 & 87.6 & 88.2 & 93.0 & 101.5 \\
\hline 1818 & 92.7 & 92.3 & 93.2 & 92.3 & 91.2 & 91.6 & 91.6 & 89.6 & 89.1 & 90.1 & 89.8 & 87.1 & 87.1 & 87.6 & 88.2 & 93.0 & 101.6 \\
\hline 1823 & 92.7 & 92.3 & 93.2 & 92.3 & 91.2 & 91.6 & 91.6 & 89.6 & 89.1 & 90.1 & 89.8 & 87.1 & 87.1 & 87.6 & 88.2 & 93.0 & 101.7 \\
\hline 1828 & 92.7 & 92.3 & 93.2 & 92.3 & 91.2 & 91.6 & 91.6 & 89.6 & 89.1 & 90.1 & 88.7 & 87.1 & 87.1 & 87.6 & 88.2 & 93.0 & 101.6 \\
\hline 1833 & 92.7 & 92.3 & 93.2 & 92.3 & 90.1 & 90.5 & 90.5 & 89.6 & 89.1 & 90.1 & 88.7 & 87.1 & 87.1 & 87.6 & 88.2 & 91.9 & 101.1 \\
\hline 1838 & 92.7 & 92.3 & 93.2 & 92.3 & 90.1 & 90.5 & 90.5 & 89.6 & 89.1 & 90.1 & 88.7 & 87.1 & 87.1 & 87.6 & 88.2 & 91.9 & 101.1 \\
\hline 1843 & 92.7 & 92.3 & 93.2 & 92.3 & 90.1 & 90.5 & 90.5 & 89.6 & 89.1 & 90.1 & 88.7 & 87.1 & 87.1 & 87.6 & 88.2 & 91.9 & 101.4 \\
\hline
\end{tabular}




\begin{tabular}{|c|c|c|c|c|c|c|c|c|c|c|c|c|c|c|c|c|c|}
\hline 1848 & 91.6 & 92.3 & 92.1 & 92.3 & 90.1 & 90.5 & 90.5 & 89.6 & 89.1 & 90.1 & 88.7 & 87.1 & 87.1 & 87.6 & 88.2 & 91.9 & 101.2 \\
\hline 1853 & 91.6 & 91.2 & 92.1 & 92.3 & 90.1 & 90.5 & 90.5 & 89.6 & 89.1 & 90.1 & 88.7 & 87.1 & 87.1 & 87.6 & 88.2 & 91.9 & 101.1 \\
\hline 1858 & 91.6 & 91.2 & 92.1 & 92.3 & 90.1 & 90.5 & 90.5 & 89.6 & 89.1 & 90.1 & 88.7 & 87.1 & 87.1 & 87.6 & 88.2 & 91.9 & 101.0 \\
\hline 863 & 91.6 & 91.2 & 92.1 & 92.3 & 90.1 & 90.5 & 90.5 & 89.6 & 89.1 & 89.1 & 88.7 & 87.1 & 87.1 & 87.6 & 88.2 & 91.9 & 101.0 \\
\hline 868 & 91.6 & 91.2 & 92.1 & 92.3 & 90.1 & 90.5 & 90.5 & 89.6 & 89.1 & 89.1 & 88.7 & 87.1 & 87.1 & 87.6 & 88.2 & 91.9 & 101. \\
\hline 873 & 91.6 & 91.2 & 92.1 & 91.2 & 90.1 & 90.5 & 90.5 & 89.6 & 89.1 & 89.1 & 88.7 & 87.1 & 87.1 & 86.5 & 88.2 & 91.9 & 100. \\
\hline 878 & 91.6 & 91.2 & 92.1 & 91.2 & 90.1 & 90.5 & 90.5 & 88.5 & 89.1 & 89.1 & 88.7 & 87.1 & 87.1 & 86.5 & 88.2 & 91.9 & 100. \\
\hline 883 & 91.6 & 91.2 & 92.1 & 91.2 & 90.1 & 90.5 & 90.5 & 88.5 & 89.1 & 89.1 & 88.7 & 87.1 & 87.1 & 86.5 & 88.2 & 91.9 & 100. \\
\hline 888 & 91.6 & 91.2 & 92.1 & 91.2 & 90.1 & 90.5 & 89.4 & 88.5 & 89.1 & 89.1 & 88.7 & 86.0 & 87.1 & 86.5 & 88.2 & 91.9 & 100. \\
\hline 893 & 91.6 & 91.2 & 92.1 & 91.2 & 89.1 & 89.4 & 89.4 & 88.5 & 89.1 & 89.1 & 88.7 & 86.0 & 87.1 & 86.5 & 88.2 & 91.9 & 100. \\
\hline 398 & 91.6 & 91.2 & 92.1 & 91.2 & 89.1 & 89.4 & 89.4 & 88.5 & 88.0 & 89.1 & 87.6 & 86.0 & 86.0 & & 87.1 & 91.9 & 100. \\
\hline 903 & 91.6 & 91.2 & 91.0 & 91.2 & 89.1 & 89.4 & 89.4 & 88.5 & 88.0 & 89.1 & 87.6 & 86.0 & 86.0 & 86.5 & 87.1 & 90.9 & 100. \\
\hline 908 & 91.6 & 91.2 & 91.0 & 91.2 & 89.1 & 89.4 & 89.4 & 88.5 & 88.0 & 89.1 & 87.6 & 86.0 & 86.0 & & 87.1 & 90.9 & 100. \\
\hline 913 & 90.5 & 91.2 & 91.0 & 91.2 & 89.1 & 89.4 & 89.4 & 88.5 & 88.0 & & 87.6 & 86.0 & 86.0 & & 87.1 & 90.9 & 100.1 \\
\hline 918 & 90.5 & 91.2 & 91.0 & 91.2 & 89.1 & 89.4 & 89.4 & 88.5 & 88.0 & & 87.6 & 86.0 & & & 87.1 & 90.9 & 100. \\
\hline 923 & 90.5 & 90.1 & 91.0 & 91.2 & 89.1 & 89. & 89.4 & 88.5 & & & 87.6 & & & & 87.1 & 90.9 & ○. \\
\hline 928 & 90.5 & 90.1 & 91.0 & 91.2 & 89.1 & 89.4 & 89.4 & 88.5 & 38.0 & 9.1 & 87.6 & 86.0 & & & 87.1 & 90.9 & \\
\hline 933 & 90.5 & 90.1 & 91.0 & 91.2 & 9.1 & 9.4 & 89.4 & 88.5 & 8.0 & 9.1 & 87.6 & 86.0 & 86.0 & 5 & 87.1 & 90.9 & 00.1 \\
\hline 938 & 90.5 & 90.1 & 91.0 & 91.2 & 89.1 & 89.4 & 89.4 & 88.5 & 88.0 & 89.1 & 87.6 & 86.0 & 86.0 & 86.5 & 87.1 & 90.9 & 100. \\
\hline 943 & 90.5 & 90.1 & 91.0 & 90.1 & 9.1 & 89.4 & 89.4 & 88.5 & 8.0 & 89.1 & 87.6 & 86.0 & 86.0 & 86.5 & 87.1 & 90.9 & 100.1 \\
\hline 948 & 90.5 & 90.1 & 91.0 & 90.1 & 9.1 & 89.4 & 89.4 & 88.5 & 88.0 & 89.1 & 87.6 & 86.0 & 86.0 & 86.5 & 87.1 & 90.9 & 99.8 \\
\hline 953 & 90.5 & 90.1 & 91.0 & 90.1 & 89.1 & 89.4 & 89.4 & 88.5 & 88.0 & 89.1 & 87.6 & 86.0 & 86.0 & 86.5 & 87.1 & 90.9 & 100. \\
\hline 958 & 90.5 & 90.1 & 91.0 & 90.1 & 9.1 & 89.4 & 89.4 & 87.4 & 88.0 & 88.0 & 87.6 & 86.0 & 86.0 & 86.5 & 87.1 & 90.9 & 99.9 \\
\hline 963 & $9 \odot .5$ & $9 \odot .1$ & $91 . \odot$ & 90.1 & 9.1 & 89.4 & 89.4 & 87.4 & 88.0 & 88.0 & 87.6 & 86.0 & 86.0 & 85.5 & 87.1 & 90.9 & 9.9 \\
\hline 968 & 90.5 & 90.1 & 91.0 & 90.1 & 89.1 & 88.3 & 88.3 & 87.4 & 86.9 & 88.0 & 87.6 & 84.9 & 86.0 & 85.5 & 87.1 & 89.9 & 9.6 \\
\hline 973 & 90.5 & 90.1 & 90.0 & 90.1 & 88.0 & 88.3 & 88.3 & 87.4 & 86.9 & 88.0 & 87.6 & 84.9 & 86.0 & 85.5 & 87.1 & 89.9 & 9.3 \\
\hline 978 & 90.5 & 90.1 & 90.0 & 90.1 & 88.0 & 88.3 & 88.3 & 87.4 & 86.9 & 88.0 & 87.6 & 84.9 & 86.0 & 85.5 & 87.1 & 89.9 & 99.3 \\
\hline 983 & 90.5 & 90.1 & 90.0 & 90.1 & 88.0 & 88.3 & 88.3 & 87.4 & 86.9 & 88.0 & 87.6 & 84.9 & 86.0 & 85.5 & 86.0 & 89.9 & 99.1 \\
\hline 988 & 89.4 & 89.1 & 90.0 & 90.1 & 88.0 & 88.3 & 88.3 & 87.4 & 86.9 & 88.0 & 86.5 & 84.9 & 84.9 & 85.5 & 86.0 & 89.9 & 99.1 \\
\hline 3 & 89.4 & 89.1 & 90.0 & 90.1 & 88.0 & 88.3 & 88.3 & 87.4 & 86.9 & 88.0 & 86.5 & 84.9 & 84.9 & 85.5 & 86.0 & 89.9 & 98.9 \\
\hline 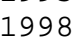 & 89.4 & 89.1 & 90.0 & 90.1 & 88.0 & 88.3 & 88.3 & 87.4 & 86.9 & 88.0 & 86.5 & 84.9 & 84.9 & 85.5 & 86.0 & 89.9 & 98.9 \\
\hline 2003 & 89.4 & 89.1 & 90.0 & 89.1 & 88.0 & 88.3 & 88.3 & 87.4 & 86.9 & 88.0 & 86.5 & 84.9 & 84.9 & 85.5 & 86.0 & 89.9 & 98.8 \\
\hline 2008 & 89.4 & 89.1 & 90.0 & 89.1 & 88.0 & 88.3 & 88.3 & 87.4 & 86.9 & 88.0 & 86.5 & 84.9 & 84.9 & 85.5 & 86.0 & 89.9 & 99.0 \\
\hline 2013 & 89.4 & 89.1 & 90.0 & 89.1 & 88.0 & 88.3 & 88.3 & 87.4 & 86.9 & 88.0 & 86.5 & 84.9 & 84.9 & 85.5 & 86.0 & 89.9 & 98.9 \\
\hline 2018 & 89.4 & 89.1 & 90.0 & 89.1 & 88.0 & 88.3 & 88.3 & 87.4 & 86.9 & 88.0 & 86.5 & 84.9 & 84.9 & 85.5 & 86.0 & 89.9 & 99.0 \\
\hline 2023 & 89.4 & 89.1 & 90.0 & 89.1 & 88.0 & 88.3 & 88.3 & 87.4 & 86.9 & 86.9 & 86.5 & 84.9 & 84.9 & 85.5 & 86.0 & 88.8 & 98.9 \\
\hline 2028 & 89.4 & 89.1 & 90.0 & 89.1 & 88.0 & 88.3 & 88.3 & 86.4 & 86.9 & 86.9 & 86.5 & 84.9 & 84.9 & 85.5 & 86.0 & 88.8 & 98.8 \\
\hline 2033 & 89.4 & 89.1 & 90.0 & 89.1 & 88.0 & 88.3 & 88.3 & 86.4 & 86.9 & 86.9 & 86.5 & 84.9 & 84.9 & 85.5 & 86.0 & 88.8 & 99.3 \\
\hline 2038 & 88.3 & 89.1 & 88.9 & 89.1 & 88.0 & 88.3 & 88.3 & 86.4 & 86.9 & 86.9 & 86.5 & 84.9 & 84.9 & 85.5 & 86.0 & 88.8 & 98.6 \\
\hline 2043 & 88.3 & 89.1 & 88.9 & 89.1 & 86.9 & 88.3 & 87.3 & 86.4 & 86.9 & 86.9 & 86.5 & 84.9 & 84.9 & 85.5 & 86.0 & 88.8 & 98.2 \\
\hline 2048 & 88.3 & 89.1 & 88.9 & 89.1 & 86.9 & 87.3 & 87.3 & 86.4 & 85.8 & 86.9 & 86.5 & 84.9 & 84.9 & 84.4 & 86.0 & 88.8 & 98.1 \\
\hline 2053 & 88.3 & 89.1 & 88.9 & 89.1 & 86.9 & 87.3 & 87.3 & 86.4 & 85.8 & 86.9 & 86.5 & 84.9 & 84.9 & 84.4 & 84.9 & 88.8 & 98.3 \\
\hline 2058 & 88.3 & 89.1 & 88.9 & 89.1 & 86.9 & 87.3 & 87.3 & 86.4 & 85.8 & 86.9 & 86.5 & 83.8 & 84.9 & 84.4 & 84.9 & 88.8 & 98.0 \\
\hline 2063 & 88.3 & 89.1 & 88.9 & 89.1 & 86.9 & 87.3 & 87.3 & 86.4 & 85.8 & 86.9 & 85.5 & 83.8 & 84.9 & 84.4 & 84.9 & 88.8 & 97.9 \\
\hline 2068 & 88.3 & 88.0 & 88.9 & 89.1 & 86.9 & 87.3 & 87.3 & 86.4 & 85.8 & 86.9 & 85.5 & 83.8 & 83.8 & 84.4 & 84.9 & 88.8 & 97.9 \\
\hline 2073 & 88.3 & 88.0 & 88.9 & 89.1 & 86.9 & 87.3 & 87.3 & 86.4 & 85.8 & 86.9 & 85.5 & 83.8 & 83.8 & 84.4 & 84.9 & 88.8 & 98.0 \\
\hline 2078 & 88.3 & 88.0 & 88.9 & 88.0 & 86.9 & 87.3 & 87.3 & 86.4 & 85.8 & 86.9 & 85.5 & 83.8 & 83.8 & 84.4 & 84.9 & 88.8 & 97.9 \\
\hline
\end{tabular}




\begin{tabular}{|c|c|c|c|c|c|c|c|c|c|c|c|c|c|c|c|c|c|}
\hline 2083 & 88.3 & 88.0 & 88.9 & 88.0 & 86.9 & 87.3 & 87.3 & 86.4 & 85.8 & 86.9 & 85.5 & 83.8 & 83.8 & 84.4 & 84.9 & 88.8 & 98.2 \\
\hline 2088 & 88.3 & 88.0 & 88.9 & 88.0 & 86.9 & 87.3 & 87.3 & 86.4 & 85.8 & 86.9 & 85.5 & 83.8 & 83.8 & 84.4 & 84.9 & 87.8 & 97.7 \\
\hline 2093 & 88.3 & 88.0 & 88.9 & 88.0 & 86.9 & 87.3 & 87.3 & 86.4 & 85.8 & 85.8 & 85.5 & 83.8 & 83.8 & 84.4 & 84.9 & 87.8 & 97.8 \\
\hline 2098 & 88.3 & 88.0 & 88.9 & 88.0 & 86.9 & 87.3 & 87.3 & 86.4 & 85.8 & 85.8 & 85.5 & 83.8 & 83.8 & 84.4 & 84.9 & 87.8 & 97.7 \\
\hline 2103 & 88.3 & 88.0 & 87.8 & 88.0 & 85.8 & 87.3 & 86.2 & 86.4 & 85.8 & 85.8 & 85.5 & 83.8 & 83.8 & 84.4 & 84.9 & 87.8 & 97.2 \\
\hline 2108 & 88.3 & 88.0 & 87.8 & 88.0 & 85.8 & 86.2 & 86.2 & 85.3 & 85.8 & 85.8 & 85.5 & 83.8 & 83.8 & 84.4 & 84.9 & 87.8 & 97.4 \\
\hline 113 & 88.3 & 88.0 & 87.8 & 88.0 & 85.8 & 86.2 & 86.2 & 85.3 & 84.7 & 85.8 & 85.5 & 83.8 & 83.8 & 84.4 & 84.9 & 87.8 & 97.4 \\
\hline 118 & 88.3 & 88.0 & 87.8 & 88.0 & 85.8 & 86.2 & 86.2 & 85.3 & 84.7 & 85.8 & 85.5 & 83.8 & 83.8 & 84.4 & 84.9 & 87.8 & 97.0 \\
\hline 123 & 87.3 & 86.9 & 87.8 & 88.0 & 85.8 & 86.2 & 86.2 & 85.3 & 84.7 & 85.8 & 85.5 & 83.8 & 83.8 & 84.4 & 84.9 & 87.8 & 97.0 \\
\hline 128 & 87.3 & 86.9 & 87.8 & 88.0 & 85.8 & 86.2 & 86.2 & 85.3 & 84.7 & 85.8 & 85.5 & 83.8 & 83.8 & 83.3 & 83.8 & 87.8 & 97.7 \\
\hline 133 & 87.3 & 86.9 & 87.8 & 86.9 & 85.8 & 86.2 & 86.2 & 85.3 & 84.7 & 85.8 & 85.5 & 83.8 & 83.8 & 83.3 & 83.8 & 87.8 & 97.1 \\
\hline 138 & 87.3 & 86.9 & 87.8 & 86.9 & 85.8 & 86.2 & 86.2 & 85.3 & 84.7 & 85.8 & 85.5 & 83.8 & 83.8 & 83.3 & 83.8 & 87.8 & 96.9 \\
\hline 143 & 87.3 & 86.9 & 87.8 & 86.9 & 85.8 & 86.2 & 86.2 & 85.3 & 84.7 & 85.8 & 85.5 & 83.8 & 83.8 & 83.3 & 83.8 & 87.8 & 97.1 \\
\hline 148 & 87.3 & 86.9 & 87.8 & 86.9 & 85.8 & 86.2 & 86.2 & 85.3 & 84.7 & 85.8 & 85.5 & 82.8 & 82.8 & 83.3 & 83.8 & & 97.5 \\
\hline 153 & 87.3 & 86.9 & 87.8 & 86.9 & 85.8 & 86.2 & 86.2 & 85.3 & 84.7 & 85.8 & 85.5 & 82.8 & 82.8 & 83.3 & 83.8 & 87.8 & 96.9 \\
\hline 158 & 87.3 & 86.9 & 87.8 & 86.9 & 85.8 & 86. & 86.2 & 85.3 & 84.7 & 85.8 & 84.4 & 82.8 & 82.8 & 83.3 & 83.8 & 86.8 & 96.7 \\
\hline 163 & 87.3 & 86.9 & 87.8 & 86.9 & 85.8 & 86.2 & 86.2 & 35.3 & 84.7 & 85.8 & 84.4 & 82.8 & 82.8 & 83.3 & 83.8 & 86.8 & 96.6 \\
\hline 168 & 87.3 & 86.9 & 87.8 & 86.9 & 85.8 & 86.2 & 86.2 & 5.3 & 84.7 & 85.8 & 34.4 & 82.8 & 82.8 & 83.3 & 83.8 & 86.8 & 96.7 \\
\hline 173 & 87.3 & 86.9 & 87.8 & 86.9 & 85.8 & 86.2 & 86.2 & 5.3 & 84.7 & 84.7 & 84.4 & 82.8 & 82.8 & 83.3 & 83.8 & 86.8 & 96.5 \\
\hline 178 & 87.3 & 86.9 & 86.7 & 86.9 & 84.7 & 86.2 & 85.1 & 5.3 & 84.7 & 84.7 & 84.4 & 82.8 & 82.8 & 83.3 & 83.8 & 86.8 & 96.4 \\
\hline 183 & 86.2 & 85.8 & 86.7 & 86.9 & 84.7 & 86.2 & 85.1 & 5.3 & 84.7 & 84.7 & 84.4 & 82.8 & 82.8 & 83.3 & 83.8 & 86.8 & 96.2 \\
\hline 188 & 86.2 & 85.8 & 86.7 & 86.9 & 84.7 & 86.2 & 85.1 & 5.3 & 84.7 & 84.7 & 84.4 & 82.8 & 82.8 & 83.3 & 83.8 & 86.8 & 96.2 \\
\hline 193 & 86.2 & 85.8 & 86.7 & 86.9 & 84.7 & 85.1 & 85.1 & 84.2 & 84.7 & 84.7 & 84.4 & 82.8 & 82.8 & 83.3 & 83.8 & 86.8 & 96.1 \\
\hline 198 & 86.2 & 85.8 & 86.7 & 85.8 & 84.7 & 85.1 & 85.1 & 84.2 & 83.7 & 84.7 & 84.4 & 82.8 & 82.8 & 83.3 & 83.8 & 86.8 & 96.1 \\
\hline 203 & 86.2 & 85.8 & 86.7 & 85.8 & 84.7 & 85.1 & 85.1 & 84.2 & 83.7 & 84.7 & 84.4 & 82.8 & 82.8 & 82.2 & 82.8 & 86.8 & 95.7 \\
\hline 208 & 86.2 & 85.8 & 86.7 & 85.8 & 84.7 & 85.1 & 85.1 & 84.2 & 83.7 & 84.7 & 84.4 & 82.8 & 82.8 & 82.2 & 82.8 & 86.8 & 95.8 \\
\hline 213 & 86.2 & 85.8 & 86.7 & 85.8 & 84.7 & 85.1 & 85.1 & 84.2 & 83.7 & 84.7 & 84.4 & 81.7 & 82.8 & 82.2 & 82.8 & 85.7 & 95.7 \\
\hline 218 & 36.2 & 85.8 & 86.7 & 85.8 & 84.7 & 85.1 & 85.1 & 84.2 & 83.7 & 84.7 & 84.4 & 81.7 & 82.8 & 82.2 & 82.8 & 85.7 & 95.9 \\
\hline 223 & 86.2 & 85.8 & 86.7 & 85.8 & 84.7 & 85.1 & 85.1 & 84.2 & 83.7 & 84.7 & 84.4 & 81.7 & 82.8 & 82.2 & 82.8 & 85.7 & 95.9 \\
\hline 228 & 6.2 & 85.8 & 86.7 & 85.8 & 84.7 & 85.1 & 85.1 & 84.2 & 83.7 & 84.7 & 83.3 & 81.7 & 81.7 & 82.2 & 82.8 & 85.7 & 95.6 \\
\hline 233 & 86.2 & 85.8 & 86.7 & 85.8 & 84.7 & 85.1 & 85.1 & 84.2 & 83.7 & 84.7 & 83.3 & 81.7 & 81.7 & 82.2 & 82.8 & 85.7 & 95.6 \\
\hline 238 & 86.2 & 85.8 & 86.7 & 85.8 & 84.7 & 85.1 & 85.1 & 84.2 & 83.7 & 84.7 & 83.3 & 81.7 & 81.7 & 82.2 & 82.8 & 85.7 & 95.4 \\
\hline 243 & 86.2 & 85.8 & 86.7 & 85.8 & 84.7 & 85.1 & 85.1 & 84.2 & 83.7 & 84.7 & 83.3 & 81.7 & 81.7 & 82.2 & 82.8 & & 95.5 \\
\hline 2248 & 86.2 & 85.8 & 85.6 & 85.8 & 84.7 & 85.1 & 84.0 & 84.2 & 83.7 & 84.7 & 83.3 & 81.7 & 81.7 & 82.2 & 82.8 & & 95.7 \\
\hline 2253 & 85.1 & 84.7 & 85.6 & 85.8 & 84.7 & 85.1 & 84.0 & 84.2 & 83.7 & 84.7 & 83.3 & 81.7 & 81.7 & 82.2 & 82.8 & & 95.3 \\
\hline 2258 & 85.1 & 84.7 & 85.6 & 85.8 & 83.7 & 84.0 & 84.0 & 84.2 & 83.7 & 83.7 & 83.3 & 81.7 & 81.7 & 82.2 & 82.8 & .7 & 95.0 \\
\hline 2263 & 85.1 & 84.7 & 85.6 & 85.8 & 83.7 & 84.0 & 84.0 & 84.2 & 83.7 & 83.7 & 83.3 & 81.7 & 81.7 & 82.2 & 82.8 & 85.7 & 95.0 \\
\hline 2268 & 85.1 & 84.7 & 85.6 & 84.7 & 83.7 & 84.0 & 84.0 & 83.1 & 83.7 & 83.7 & 83.3 & 81.7 & 81.7 & 82.2 & 82.8 & 85.7 & 94.9 \\
\hline 2273 & 85.1 & 84.7 & 85.6 & 84.7 & 83.7 & 84.0 & 84.0 & 83.1 & 83.7 & 83.7 & 83.3 & 81.7 & 81.7 & 82.2 & 82.8 & 85.7 & 95.0 \\
\hline 2278 & 85.1 & 84.7 & 85.6 & 84.7 & 83.7 & 84.0 & 84.0 & 83.1 & 83.7 & 83.7 & 83.3 & 81.7 & 81.7 & 82.2 & 82.8 & 85.7 & 95.1 \\
\hline 2283 & 85.1 & 84.7 & 85.6 & 84.7 & 83.7 & 84.0 & 84.0 & 83.1 & 83.7 & 83.7 & 83.3 & 81.7 & 81.7 & 82.2 & 82.8 & 85.7 & 94.9 \\
\hline 2288 & 85.1 & 84.7 & 85.6 & 84.7 & 83.7 & 84.0 & 84.0 & 83.1 & 82.6 & 83.7 & 83.3 & 81.7 & 81.7 & 82.2 & 82.8 & 84.7 & 94.8 \\
\hline 2293 & 85.1 & 84.7 & 85.6 & 84.7 & 83.7 & 84.0 & 84.0 & 83.1 & 82.6 & 83.7 & 83.3 & 81.7 & 81.7 & 81.1 & 81.7 & 84.7 & 95.2 \\
\hline 2298 & 85.1 & 84.7 & 85.6 & 84.7 & 83.7 & 84.0 & 84.0 & 83.1 & 82.6 & 83.7 & 83.3 & 81.7 & 81.7 & 81.1 & 81.7 & 84.7 & 94.6 \\
\hline 303 & 85.1 & 84.7 & 85.6 & 84.7 & 83.7 & 84.0 & 84.0 & 83.1 & 82.6 & 83.7 & 83.3 & 81.7 & 81.7 & 81.1 & 81.7 & 84.7 & 94.7 \\
\hline 308 & 85.1 & 84.7 & 84.6 & 84.7 & 83.7 & 84.0 & 84.0 & 83.1 & 82.6 & 83.7 & 83.3 & 81.7 & 81.7 & 81.1 & 81.7 & 84.7 & 94.4 \\
\hline 313 & 85.1 & 84.7 & 84.6 & 84.7 & 83.7 & 84.0 & 84.0 & 83.1 & 82.6 & 83.7 & 83.3 & 81.7 & 81.7 & 81.1 & 81.7 & 84.7 & 94.5 \\
\hline
\end{tabular}




\begin{tabular}{|c|c|c|c|c|c|c|c|c|c|c|c|c|c|c|c|c|c|}
\hline 2318 & 85.1 & 84.7 & 84.6 & 84.7 & 83.7 & 84.0 & 84.0 & 83.1 & 82.6 & 83.7 & 82.2 & 80.6 & 81.7 & 81.1 & 81.7 & 84.7 & 94.5 \\
\hline 2323 & 85.1 & 84.7 & 84.6 & 84.7 & 83.7 & 84.0 & 82.9 & 83.1 & 82.6 & 83.7 & 82.2 & 80.6 & 81.7 & 81.1 & 81.7 & 84.7 & 94.6 \\
\hline 328 & 84.0 & 84.7 & 84.6 & 84.7 & 83.7 & 84.0 & 82.9 & 83.1 & 82.6 & 83.7 & 82.2 & 80.6 & 80.6 & 81.1 & 81.7 & 84.7 & 94.3 \\
\hline 333 & 84.0 & 84.7 & 84.6 & 84.7 & 83.7 & 82.9 & 82.9 & 83.1 & 82.6 & 83.7 & 82.2 & 80.6 & 80.6 & 81.1 & 81.7 & 84.7 & 94.3 \\
\hline 338 & 84.0 & 84.7 & 84.6 & 84.7 & 83.7 & 82.9 & 82.9 & 83.1 & 82.6 & 83.7 & 82.2 & 80.6 & 80.6 & 81.1 & 81.7 & 84.7 & 94.3 \\
\hline 343 & 84.0 & 83.7 & 84.6 & 84.7 & 82.6 & 82.9 & 82.9 & 83.1 & 82.6 & 83.7 & 82.2 & 80.6 & 80.6 & 81.1 & 81.7 & 84.7 & 94.1 \\
\hline 348 & 84.0 & 83.7 & 84.6 & 84.7 & 82.6 & 82.9 & 82.9 & 83.1 & 82.6 & 82.6 & 82.2 & 80.6 & 80.6 & 81.1 & 81.7 & 84.7 & 94.0 \\
\hline 353 & 84.0 & 83.7 & 84.6 & 83.7 & 82.6 & 82.9 & 82.9 & 83.1 & 82.6 & 82.6 & 82.2 & 80.6 & 80.6 & 81.1 & 81.7 & 84.7 & 93.7 \\
\hline 358 & 84.0 & 83.7 & 84.6 & 83.7 & 82.6 & 82.9 & 82.9 & 83.1 & 82.6 & 82.6 & 82.2 & 80.6 & 80.6 & 81.1 & 81.7 & 84.7 & 93.8 \\
\hline 363 & 84.0 & 83.7 & 84.6 & 83.7 & 82.6 & 82.9 & 82.9 & 82.0 & 82.6 & 82.6 & 82.2 & 80.6 & 80.6 & 81.1 & 81.7 & 83.7 & 93.9 \\
\hline 368 & 84.0 & 83.7 & 84.6 & 83.7 & 82.6 & 82.9 & 82.9 & 82.0 & 82.6 & 82.6 & 82.2 & 80.6 & 80.6 & 81.1 & 81.7 & 83.7 & 93.8 \\
\hline 373 & 84.0 & 83.7 & 84.6 & 83.7 & 82.6 & 82.9 & 82.9 & 82.0 & 82.6 & 82.6 & 82.2 & 80.6 & 80.6 & 81.1 & 81.7 & 83.7 & 94.0 \\
\hline 378 & 84.0 & 83.7 & 84.6 & 83.7 & 82.6 & 82.9 & 82.9 & 82.0 & 82.6 & 82.6 & 82.2 & 80.6 & 80.6 & 81.1 & 80.6 & 83.7 & 93.8 \\
\hline 383 & 84.0 & 83.7 & 84.6 & 83.7 & 82.6 & 82.9 & 82.9 & 82.0 & 82.6 & 82.6 & 82.2 & 80.6 & 80.6 & 81.1 & 80.6 & 83.7 & 93.6 \\
\hline 388 & 84.0 & 83.7 & 84.6 & 83.7 & 82.6 & 82.9 & 82.9 & 82.0 & 81.5 & 82.6 & 82.2 & 80.6 & 80.6 & 81.1 & 80.6 & 83.7 & 93.7 \\
\hline 393 & 84.0 & 83.7 & 84.6 & 83.7 & 82.6 & 82.9 & 82.9 & 82.0 & 81.5 & 82.6 & 82.2 & 80.6 & 80.6 & 81.1 & 80.6 & 83.7 & 93.2 \\
\hline 2398 & 84.0 & 83.7 & 83.5 & 83.7 & 82.6 & 82.9 & 82.9 & 82.0 & 81.5 & 82.6 & 82.2 & 80.6 & 80.6 & 81.1 & 80.6 & 83.7 & 93.7 \\
\hline 403 & 84.0 & 83.7 & 83.5 & 83.7 & 82.6 & 82.9 & 82.9 & 82.0 & 81.5 & 82.6 & 82.2 & 80.6 & 80.6 & 80.1 & 80.6 & 83.7 & 93.6 \\
\hline 408 & 84.0 & 83.7 & 83.5 & 83.7 & 82.6 & 82.9 & 82.9 & 82.0 & 81.5 & 82.6 & 82.2 & 80.6 & 80.6 & 80.1 & 80.6 & 83.7 & 93.5 \\
\hline 413 & 82.9 & 82.6 & 83.5 & 83.7 & 82.6 & 82.9 & 82.9 & 82.0 & 81.5 & 82.6 & 82.2 & 80.6 & 80.6 & 80.1 & 80.6 & 83.7 & 92.9 \\
\hline 418 & 82.9 & 82.6 & 83.5 & 83.7 & 82.6 & 82.9 & 82.9 & 82.0 & 81.5 & 82.6 & 82.2 & 80.6 & 80.6 & 80.1 & 80.6 & 83.7 & 94.0 \\
\hline 423 & 82.9 & 82.6 & 83.5 & 83.7 & 82.6 & 82.9 & 82.9 & 82.0 & 81.5 & 82.6 & 82.2 & 80.6 & 80.6 & 80.1 & 80.6 & 83.7 & 93.5 \\
\hline 428 & 82.9 & 82.6 & 83.5 & 83.7 & 82.6 & 82.9 & 81.9 & 82.0 & 81.5 & 82.6 & 81.1 & 80.6 & 80.6 & 80.1 & 80.6 & 83.7 & 93.3 \\
\hline 433 & 82.9 & 82.6 & 83.5 & 83.7 & 81.5 & 81.9 & 81.9 & 82.0 & 81.5 & 82.6 & 81.1 & 80.6 & 80.6 & 80.1 & 80.6 & 83.7 & 93.2 \\
\hline 438 & 82.9 & 82.6 & 83.5 & 82.6 & 81.5 & 81.9 & 81.9 & 82.0 & 81.5 & 82.6 & 81.1 & 79.5 & 80.6 & 80.1 & 80.6 & 83.7 & 92.9 \\
\hline 443 & 82.9 & 82.6 & 83.5 & 82.6 & 81.5 & 81.9 & 81.9 & 82.0 & 81.5 & 82.6 & 81.1 & 79.5 & 80.6 & 80.1 & 80.6 & 83.7 & 93.0 \\
\hline 448 & 82.9 & 82.6 & 83.5 & 82.6 & 81.5 & 81.9 & 81.9 & 82.0 & 81.5 & 82.6 & 81.1 & 79.5 & 79.5 & 80.1 & 80.6 & 82.6 & 93.4 \\
\hline 453 & 82.9 & 82.6 & 83.5 & 82.6 & 81.5 & 81.9 & 81.9 & 82.0 & 81.5 & 81.5 & 81.1 & 79.5 & 79.5 & 80.1 & 80.6 & 82.6 & 92.8 \\
\hline 458 & 82.9 & 82.6 & 83.5 & 82.6 & 81.5 & 81.9 & 81.9 & 82.0 & 81.5 & 81.5 & 81.1 & 79.5 & 79.5 & 80.1 & 80.6 & 82.6 & 92.5 \\
\hline 463 & 82.9 & 82.6 & 83.5 & 82.6 & 81.5 & 81.9 & 81.9 & 82.0 & 81.5 & 81.5 & 81.1 & 79.5 & 79.5 & 80.1 & 80.6 & 82.6 & 92.8 \\
\hline 468 & 82.9 & 82.6 & 83.5 & 82.6 & 81.5 & 81.9 & 81.9 & 81.0 & 81.5 & 81.5 & 81.1 & 79.5 & 79.5 & 80.1 & 80.6 & 82.6 & 92.8 \\
\hline 473 & 82.9 & 82.6 & 83.5 & 82.6 & 81.5 & 81.9 & 81.9 & 81.0 & 81.5 & 81.5 & 81.1 & 79.5 & 79.5 & 80.1 & 80.6 & 82.6 & 92.8 \\
\hline 478 & 82.9 & 82.6 & 83.5 & 82.6 & 81.5 & 81.9 & 81.9 & 81.0 & 81.5 & 81.5 & 81.1 & 79.5 & 79.5 & 80.1 & 80.6 & 82.6 & 92.8 \\
\hline 483 & 82.9 & 82.6 & 82.4 & 82.6 & 81.5 & 81.9 & 81.9 & 81.0 & 81.5 & 81.5 & 81.1 & 79.5 & 79.5 & 80.1 & 80.6 & 82.6 & 92.3 \\
\hline & 82.9 & 82.6 & 82.4 & 82.6 & 81.5 & 81.9 & 81.9 & 81.0 & 81.5 & 81.5 & 81.1 & 79.5 & 79.5 & 80.1 & 80.6 & 82.6 & 92.8 \\
\hline & 82.9 & 82.6 & 82.4 & 82.6 & 81.5 & 81.9 & 81.9 & 81.0 & 80.4 & 81.5 & 81.1 & 79.5 & 79.5 & 80.1 & 79.5 & 82.6 & 92.3 \\
\hline 498 & 82.9 & 82.6 & 82.4 & 82.6 & 81.5 & 81.9 & 81.9 & 81.0 & 80.4 & 81.5 & 81.1 & 79.5 & 79.5 & 80.1 & 79.5 & 82.6 & 92.5 \\
\hline
\end{tabular}




\subsection{Wall Thermocouple and Plate Thermometer Data}

Note: The data below indicates considerable differences between the temperatures measured on the north and west walls. The former appear to closely follow the temperatures measured for the structural steel column while the latter somewhat follow the furnace temperature curve, but with enhanced heating (higher temperatures) at early times. The reasons for these discrepancies are unknown at this time and resolving them was beyond the scope of the present study. It is therefore recommended that the wall temperature data be used with caution. Conversely, the values measured for the two plate thermometers appear quite reasonable in comparison to the average conventionally measured furnace temperature.

\begin{tabular}{|c|c|c|c|c|c|c|c|c|c|c|}
\hline \multirow{4}{*}{$\begin{array}{l}\text { Time } \\
\text { hr:min:sec } \\
\odot: \odot \odot: \odot \odot\end{array}$} & \multicolumn{3}{|c|}{ North wall } & \multicolumn{3}{|c|}{ West wall } & \multicolumn{4}{|c|}{ Plate thermometers } \\
\hline & Time & TC \#19 & TC \#20 & AVE. & TC \#21 & TC \#22 & AVE. & $\mathrm{C} \# 23 \mathrm{~T}$ & TC \#24 & AVE \\
\hline & Min & $\operatorname{Deg} . F$ & $\operatorname{Deg} . \mathrm{F}$ & $\operatorname{Deg} \cdot \mathrm{F}$ & $\operatorname{Deg} . F$ & $\mathrm{Deg} \cdot \mathrm{F}$ & $\operatorname{Deg} \cdot F \quad$ & $\operatorname{Deg} . F \quad$ & $\operatorname{Deg} \cdot \mathrm{F}$ & Deg.F \\
\hline & $\odot$ & 68.8 & 67.2 & 68.0 & 108.3 & 219.0 & 163.7 & 91.6 & 82.8 & 87.2 \\
\hline$\odot: \odot 1: \odot \odot$ & 1 & 78.8 & 75.5 & 77.1 & 231.6 & 503.2 & 367.4 & 160.0 & 131.2 & 145.6 \\
\hline$\odot: \odot 2: \odot \odot$ & 2 & 127.4 & 108.7 & 118.1 & $750 . \odot$ & 928.2 & 839.1 & 550.6 & 394.2 & 472.4 \\
\hline$\odot: \odot 3: \odot \odot$ & 3 & 168.8 & 144.4 & 156.6 & 898.0 & 1038.4 & 968.2 & 846.9 & 643.1 & 745.0 \\
\hline$\odot: \odot 4: \odot \odot$ & 4 & 206.5 & 176.8 & 191.6 & 966.6 & 1043.8 & 1005.2 & 1038.6 & 812.7 & 925.6 \\
\hline$\odot: \odot 5: \odot \odot$ & 5 & 237.6 & 202.1 & 219.9 & 961.3 & 1017.3 & 989.3 & 1135.8 & 3913.1 & 1024.4 \\
\hline$\odot: \odot 6: \odot \odot$ & 6 & 277.8 & 236.5 & 257.2 & 935.1 & 1011.4 & 973.2 & 1233.7 & 71059.4 & 1146.6 \\
\hline$\odot: \odot 7: \odot \odot$ & 7 & 311.4 & 267.0 & 289.2 & 932.0 & 992.8 & 962.4 & 1282.3 & 31131.3 & 1206.8 \\
\hline ๑:०८:०० & 8 & 339.2 & 294.2 & 316.7 & 964.9 & 975.0 & 970.0 & 1244.1 & 11150.2 & 1197.1 \\
\hline$\odot: \odot 9: \odot \odot$ & 9 & 371.4 & 322.5 & 346.9 & 959.0 & 1052.8 & 1005.9 & 1256.9 & 91205.2 & 1231.1 \\
\hline$\odot: 1 \odot: \odot \odot$ & 10 & 403.6 & 352.5 & 378.1 & 973.9 & 1080.7 & 1027.3 & 31312.7 & 71254.9 & 1283.8 \\
\hline$\odot: 11: 0 \odot$ & 11 & 437.0 & 384.0 & 410.5 & 1073.7 & 1089.3 & 1081.5 & 51312.0 & 1255.3 & 1283.6 \\
\hline$: 12: \odot \odot$ & 12 & 469.7 & 414.1 & 441.9 & 1052.1 & 1122.8 & 1087.4 & 1340.8 & 31259.8 & 1300.3 \\
\hline : ๑९ & 13 & 503.6 & 443.1 & 473.3 & 1087.2 & 1170.5 & 1128.8 & 31373.2 & 21268.6 & 1320.9 \\
\hline$\odot \odot$ & 14 & 540.5 & 477.1 & 508.8 & 1146.0 & 1211.2 & 1178.6 & 1424.3 & 31306.9 & 1365.6 \\
\hline$\odot: 15: \odot \odot$ & 15 & 576.5 & 509.2 & 542.8 & 1225.8 & 1207.0 & 1216.4 & +1453.3 & 31303.2 & 1378.2 \\
\hline$\odot: 16: \odot \odot$ & 16 & 618.0 & 541.9 & 580.0 & 1291.6 & 1312.9 & 1302.3 & 31491.3 & 31357.9 & 1424.6 \\
\hline$\odot: 17: \odot \odot$ & 17 & 665.6 & 594.3 & 629.9 & 1334.7 & 1323.3 & 1329.0 & 1489.5 & 51452.2 & 1470.8 \\
\hline$\odot: 18: \odot \odot$ & 18 & 712.5 & 647.6 & 680.1 & 1351.0 & 1359.7 & 1355.4 & 1470.6 & 1507.6 & 1489.1 \\
\hline$: \odot \odot$ & 19 & 760.7 & 698.3 & 729.5 & 1361.7 & 1371.2 & 1366.4 & 1477. & 41504. & 1491.0 \\
\hline : ๑९ & 20 & 815.0 & 755.3 & 785.2 & 1360.9 & 1374.1 & 1367.5 & 51466.1 & 114 & 1476.1 \\
\hline$\odot: 21: \odot \odot$ & 21 & 863.4 & 802.2 & 832.8 & 1379.5 & 1385.8 & 1382.6 & 1487.3 & 31477.4 & 1482.4 \\
\hline$\odot: 22: \odot \odot$ & 22 & 902.9 & 834.1 & 868.5 & 1351.8 & 1339.3 & 1345.6 & 1484.6 & 1406.3 & 1445.5 \\
\hline$\odot: 23: \odot \odot$ & 23 & 943.6 & 867.1 & 905.3 & 1413.7 & 1416.0 & 1414.9 & 1519 . & 31405.0 & 1462.2 \\
\hline$\odot: 24: \odot \odot$ & 24 & 988.4 & 904.2 & 946.3 & 1390.6 & 1401.1 & 1395.9 & 1538.2 & 1425.2 & 1481.7 \\
\hline : ๑९ & 25 & 1036.2 & 951.7 & 993.9 & 1434.0 & 1414.8 & 1424.4 & 1548.7 & 71440.7 & 1494.7 \\
\hline$\odot: 2$ & 26 & 1080.0 & 994.2 & 1037.1 & 1429.0 & 1410.3 & 1419.6 & 1558. & 1447.2 & 1502.6 \\
\hline$: \odot \odot$ & 27 & 1117.7 & 1030.0 & 1073.8 & 1427.7 & 1427.7 & 1427.7 & 71573.0 & 1434.4 & 1503.7 \\
\hline$\odot: 28: \odot \odot$ & 28 & 1153.1 & 1065.4 & 1109.2 & 1419.1 & 1419.4 & 1419.3 & 31573.7 & 71432.8 & 1503.2 \\
\hline$\odot: 29: \odot \odot$ & 29 & 1183.9 & 1097.2 & 1140.5 & 1412.1 & 1429.0 & 1420.5 & 51580.2 & 21438.3 & 1509.3 \\
\hline$\odot: 30: \odot \odot$ & 30 & 1216.7 & 1130.8 & 1173.8 & 1402.3 & 1416.6 & 1409.5 & 1564.9 & 1462.3 & 1513.6 \\
\hline$\odot \odot$ & 31 & 1247.5 & 1163.6 & 1205.6 & 1441.8 & 1444.5 & 1443.1 & 1559.7 & 71481.2 & 1520.4 \\
\hline$\odot \odot$ & 32 & 12 & 1192.4 & 1232.9 & 1449.7 & 1443.9 & 1446.8 & 31555.0 & 1501.5 & 1528.3 \\
\hline $0: 33$ & 33 & 1300.5 & 1221.2 & 1260.9 & 1438.9 & 1464.3 & 1451.6 & 15 & 31515.9 & 1539.9 \\
\hline$\odot: 34: 0 \odot$ & 34 & 1322.8 & 1246.7 & 1284.7 & 1452.7 & 1481.2 & 1467.0 & 1569.0 & 1518.4 & 1543.7 \\
\hline$\odot: 35: \odot \odot$ & 35 & 1342.0 & 1269.3 & 1305.6 & 1483.5 & 1462.6 & 1473.1 & 1583.1 & 11528.3 & 1555.7 \\
\hline$\odot: 36: \odot \odot$ & 36 & 1361.4 & 1291.7 & 1326.5 & 1487.1 & 1477.4 & 1482.3 & 31586.5 & 51531.0 & 1558.8 \\
\hline : ๑९ & 37 & 1377.4 & 1312.7 & 1345.0 & 1507.5 & 1486.9 & 1497.2 & 1596.9 & 91537.2 & 1567.0 \\
\hline$\odot \odot$ & 38 & 1397.5 & 1334.3 & 1365.9 & 1493.6 & 1513.9 & 1503.8 & 1596.6 & 1552.3 & 1574.4 \\
\hline$\odot: 39: \odot \odot$ & 39 & 1413.3 & 1353.1 & 1383.2 & 1512.5 & 1503.9 & 1508.2 & 21608.4 & 41553.7 & 1581.1 \\
\hline$\odot: 4 \odot: \odot \odot$ & 40 & 1432.0 & 1370.8 & 1401.4 & 1511.1 & 1516.5 & 1513.8 & 31612.0 & פ 1563.3 & 1587.7 \\
\hline$\odot: 41: \odot \odot$ & 41 & 1450.0 & 1386.5 & 1418.3 & 1510.9 & 1514.7 & 1512.8 & 31614.4 & 41571.5 & 1593.0 \\
\hline$\odot: 42: \odot \odot$ & 42 & 1469.1 & 1404.2 & 1436.6 & 1516.6 & 1517.9 & 1517.3 & 31617.1 & 11582.9 & 1600.0 \\
\hline & 43 & 1486.5 & 1420.2 & 1453.4 & 1549.8 & 1539.9 & 1544.8 & 31629.5 & 51589.0 & 1609.3 \\
\hline$\odot: 44: \odot \odot$ & 44 & 1505.3 & 1437.7 & 1471.5 & 1535.9 & 1538.6 & 1537.3 & 31620.1 & 11611.7 & 1615.9 \\
\hline
\end{tabular}




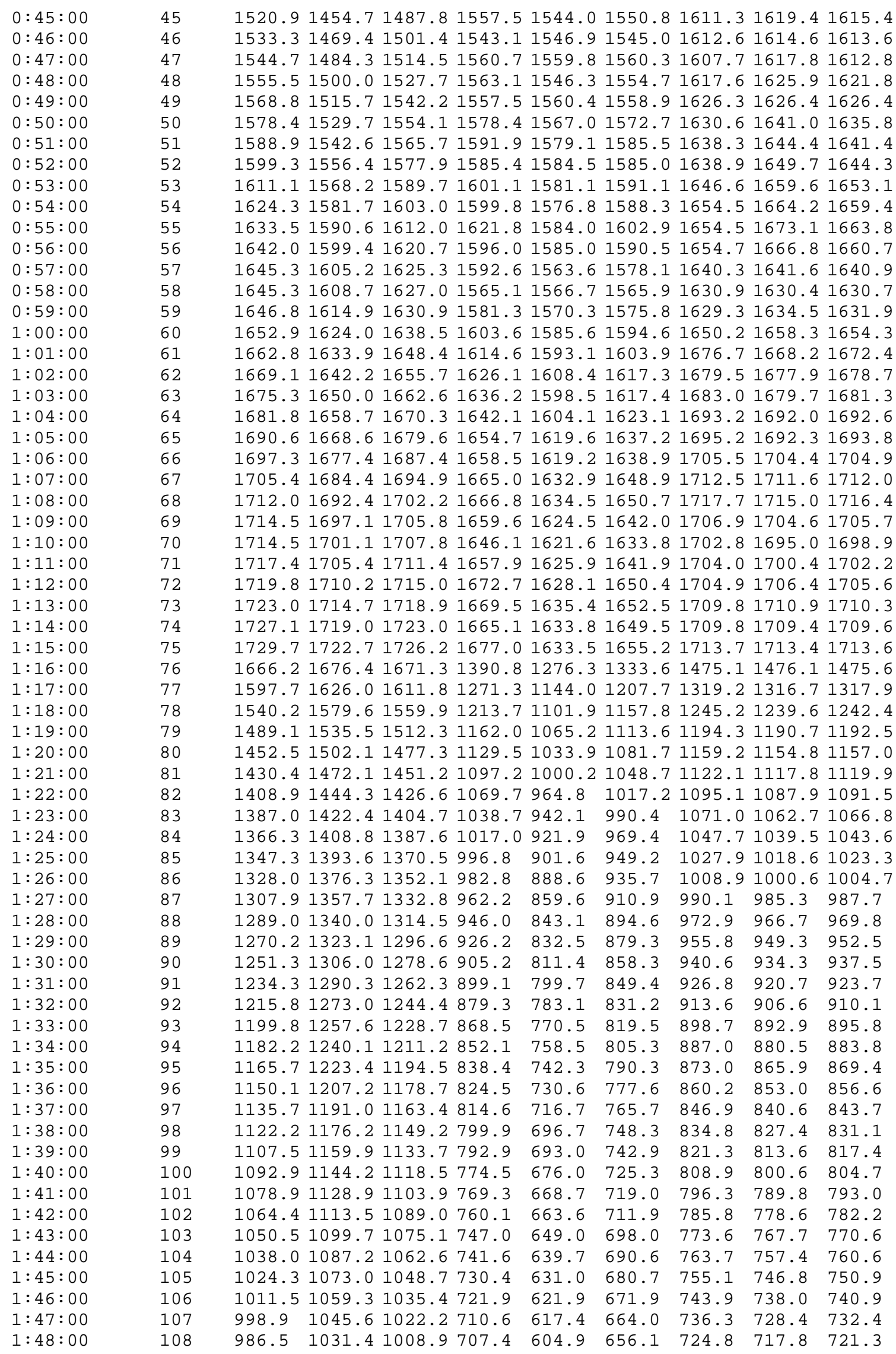




\begin{tabular}{|c|c|c|c|c|c|c|c|c|c|c|}
\hline $1: 49: \odot \odot$ & 109 & 975.2 & 1018.6 & 996.9 & 699.4 & 600.3 & 649.8 & 717.3 & 710.1 & 713.7 \\
\hline $1: 50: \odot \odot$ & 110 & 963.1 & 1005.2 & 984.2 & 686.1 & 591.6 & 638.9 & 708.8 & 701.2 & 705.0 \\
\hline $1: 51: \odot \odot$ & 111 & 952.5 & 992.4 & 972.5 & 680.5 & 585.9 & 633.2 & 698.4 & 692.8 & 695.6 \\
\hline $1: 52: \odot \odot$ & 112 & 940.5 & 979.8 & 960.1 & 671.4 & 574.5 & 622.9 & 689.9 & 683.2 & 686.6 \\
\hline $1: 53: \odot \odot$ & 113 & 929.4 & 967.5 & 948.4 & 662.5 & 566.6 & 614.6 & 683.1 & 675.3 & 679.2 \\
\hline $1: 54: \odot \odot$ & 114 & 918.3 & 955.2 & 936.7 & 659.5 & 560.5 & 610.0 & 672.3 & 665.8 & 669.0 \\
\hline $1: 55: \odot \odot$ & 115 & 908.5 & 944.3 & 926.4 & 655.0 & 555.6 & 605.3 & 664.5 & 657.7 & 661.1 \\
\hline $1: 56: \odot \odot$ & 116 & 898.7 & 932.1 & 915.4 & 647.6 & 545.9 & 596.8 & 655.0 & 650.8 & 652.9 \\
\hline $1: 57: \odot \odot$ & 117 & 888.8 & 921.1 & 904.9 & 642.0 & 544.8 & 593.4 & 647.1 & 642.2 & 644.6 \\
\hline $1: 58: \odot \odot$ & 118 & 877.9 & 910.1 & 894.0 & 631.8 & 537.1 & 584.4 & 639.9 & 633.9 & 636.9 \\
\hline $1: 59: \odot \odot$ & 119 & 867.8 & 899.1 & 883.5 & 624.0 & 528.6 & 576.3 & 631.0 & 625.3 & 628.2 \\
\hline $2: \odot \odot: \odot \odot$ & 120 & 858.4 & 888.7 & 873.5 & 618.3 & 524.3 & 571.3 & 623.7 & 620.2 & 622.0 \\
\hline$: 01: 00$ & 121 & 849.2 & 878.6 & 863.9 & 613.0 & 531.5 & 572.3 & 617.4 & 613.6 & 615.5 \\
\hline $2: \odot 2: \odot \odot$ & 122 & 841.1 & 868.5 & 854.8 & 607.5 & 512.1 & 559.8 & 609.4 & 604.9 & 607.2 \\
\hline 2:๑3:๑९ & 123 & 831.6 & 858.8 & 845.2 & 600.3 & 507.7 & 554.0 & 601.7 & 597.7 & 599.7 \\
\hline $2: \odot 4: \odot \odot$ & 124 & 822.7 & 849.3 & 836.0 & 596.7 & 500.5 & 548.6 & 595.4 & 591.8 & 593.6 \\
\hline $2: \odot 5: \odot \odot$ & 125 & 813.9 & 839.7 & 826.8 & 592.9 & 503.1 & 548.0 & 587.7 & 585.9 & 586.8 \\
\hline $2: \odot 6: \odot \odot$ & 126 & 806.8 & 830.8 & 818.8 & 590.7 & 490.8 & 540.8 & 582.1 & 578.3 & 580.2 \\
\hline $2: \odot 7: \odot \odot$ & 127 & 798.3 & 822.7 & 810.5 & 582.1 & 489.6 & 535.8 & 575.6 & 573.1 & 574.3 \\
\hline $2: \odot ৪: \odot \odot$ & 128 & 789.9 & 813.9 & 801.9 & 576.0 & 485.6 & 530.8 & 568.8 & 567.1 & 568.0 \\
\hline $2: 09: \odot \odot$ & 129 & 782.2 & 804.5 & 793.3 & 572.2 & 483.6 & 527.9 & 563.2 & 561.0 & 562.1 \\
\hline $2: 10: \odot \odot$ & 130 & 773.4 & 795.8 & 784.6 & 568.0 & 475.2 & 521.6 & 556.2 & 554.9 & 555.5 \\
\hline $2: 11: \odot \odot$ & 131 & 765.8 & 787.7 & 776.7 & 561.9 & 476.6 & 519.3 & 551.5 & 549.3 & 550.4 \\
\hline $2: 12: \odot \odot$ & 132 & 758.6 & 780.0 & 769.3 & 559.6 & 467.4 & 513.5 & 544.6 & 544.3 & 544.5 \\
\hline $2: 13: \odot \odot$ & 133 & 752.1 & 772.8 & 762.5 & 553.8 & 461.8 & 507.8 & 539.6 & 538.0 & 538.8 \\
\hline $2: 14: \odot \odot$ & 134 & 745.1 & 765.0 & 755.0 & 549.7 & 459.5 & 504.6 & 533.5 & 533.1 & 533.3 \\
\hline $2: 15: \odot \odot$ & 135 & 738.1 & 756.7 & 747.4 & 544.3 & 458.4 & 501.4 & 527.9 & 527.2 & 527.5 \\
\hline $2: 16: \odot \odot$ & 136 & 731.2 & 749.0 & 740.1 & 542.7 & 453.2 & 497.9 & 523.0 & 523.6 & 523.3 \\
\hline $2: 17: \odot \odot$ & 137 & 723.2 & 742.0 & 732.6 & 536.9 & 456.1 & 496.5 & 518.7 & 517.8 & 518.3 \\
\hline $2: 18: \odot \odot$ & 138 & 717.5 & 735.7 & 726.6 & 532.0 & 450.9 & 491.5 & 513.3 & 512.8 & 513.0 \\
\hline $2: 19: \odot \odot$ & 139 & 710.7 & 728.8 & 719.8 & 527.2 & 444.2 & 485.7 & 508.5 & 508.3 & 508.4 \\
\hline $2: 20: \odot \odot$ & 140 & 703.6 & 722.0 & 712.8 & 526.5 & 440.6 & 483.5 & 503.1 & 503.4 & 503.2 \\
\hline $2: 21: \odot \odot$ & 141 & $698 . \odot$ & 714.3 & 706.2 & 524.1 & 434.8 & 479.5 & 498.6 & 499.3 & 498.9 \\
\hline $2: 22: 0 \odot$ & 142 & 690.9 & 707.7 & 699.3 & 517.6 & 434.1 & 475.9 & 493.5 & 494.1 & 493.8 \\
\hline $2: 23: \odot \odot$ & 143 & 686.0 & 702.4 & 694.2 & 514.8 & 434.1 & 474.4 & 489.7 & 489.4 & 489.6 \\
\hline $2: 24: \odot \odot$ & 144 & 679.9 & 695.8 & 687.8 & 514.0 & 427.3 & 470.7 & 484.5 & 484.9 & 484.7 \\
\hline $2: 25: \odot \odot$ & 145 & 674.0 & 689.5 & 681.7 & 507.2 & 429.1 & 468.1 & 480.4 & 480.4 & 480.4 \\
\hline $2: 26: \odot \odot$ & 146 & 667.3 & 682.6 & 674.9 & 507.2 & 418.5 & 462.8 & 474.8 & 476.2 & 475.5 \\
\hline $2: 27: \odot \odot$ & 147 & 662.6 & 676.6 & 669.6 & 499.3 & 412.9 & 456.1 & 469.8 & 471.4 & 470.6 \\
\hline $2: 28: \odot \odot$ & 148 & 657.1 & 670.7 & 663.9 & 496.9 & 406.4 & 451.7 & 466.3 & 467.2 & 466.8 \\
\hline $2: 29: \odot \odot$ & 149 & 651.5 & 665.7 & 658.6 & 494.6 & 409.5 & 452.0 & 460.8 & 463.3 & 462.0 \\
\hline $2: 30: \odot \odot$ & 150 & 646.1 & 660.1 & 653.1 & 490.3 & 408.9 & 449.6 & 458.1 & 459.3 & 458.7 \\
\hline $2: 31: \odot \odot$ & 151 & 641.0 & 654.7 & 647.8 & 489.7 & 404.1 & 446.9 & 453.4 & 455.4 & 454.4 \\
\hline $2: 32: \odot \odot$ & 152 & 635.8 & 649.3 & 642.5 & 484.9 & 408.4 & 446.6 & 448.9 & 452.7 & 450.8 \\
\hline $2: 33: \odot \odot$ & 153 & 630.8 & 643.9 & 637.3 & 483.4 & 403.7 & 443.6 & 447.1 & 449.1 & 448.1 \\
\hline $2: 34: \odot \odot$ & 154 & 625.7 & 638.5 & 632.1 & 479.5 & 400.8 & 440.2 & 442.9 & 445.3 & 444.1 \\
\hline $2: 35: \odot \odot$ & 155 & 620.5 & 633.4 & 626.9 & 481.5 & 396.0 & 438.7 & 439.2 & 441.9 & 440.5 \\
\hline $2: 36: \odot \odot$ & 156 & 615.9 & 628.3 & 622.1 & 476.2 & 398.7 & 437.5 & 437.9 & 439.0 & 438.4 \\
\hline $2: 37: \odot \odot$ & 157 & 611.2 & 623.4 & 617.3 & 475.2 & 396.0 & 435.6 & 433.8 & 434.8 & 434.3 \\
\hline $2: 38: 00$ & 158 & 606.6 & 618.5 & 612.5 & 471.2 & 392.4 & 431.8 & 429.6 & 432.5 & 431.1 \\
\hline $2: 39: \odot \odot$ & 159 & $6 \odot 2 . \odot$ & 613.5 & 607.7 & 468.1 & 389.5 & 428.8 & 427.6 & 428.5 & 428.1 \\
\hline $2: 4 \odot: \odot \odot$ & 160 & 597.4 & 608.5 & 602.9 & 465.3 & 383.0 & 424.1 & 424.9 & 424.2 & 424.6 \\
\hline $2: 41: \odot \odot$ & 161 & 592.9 & 603.9 & 598.4 & 460.9 & 381.9 & 421.4 & 420.6 & 420.6 & 420.6 \\
\hline $2: 42: \odot \odot$ & 162 & 588.4 & 598.9 & 593.6 & 460.0 & 380.1 & 420.1 & 416.3 & 417.4 & 416.8 \\
\hline $2: 43: \odot \odot$ & 163 & 584.2 & 594.9 & 589.6 & 455.4 & 377.4 & 416.4 & 413.6 & 414.7 & 414.1 \\
\hline $2: 44: \odot \odot$ & 164 & 580.0 & 590.3 & 585.2 & 452.5 & 375.1 & 413.8 & 411.1 & 410.5 & 410.8 \\
\hline $2: 45: \odot \odot$ & 165 & 575.9 & 585.9 & 580.9 & 451.9 & 372.7 & 412.3 & 407.3 & 408.2 & 407.8 \\
\hline $2: 46: 00$ & 166 & 571.5 & 581.4 & 576.4 & 450.7 & 369.7 & 410.2 & 404.6 & 405.0 & 404.8 \\
\hline $2: 47: \odot \odot$ & 167 & 567.6 & 577.4 & 572.5 & 447.3 & 372.7 & 410.0 & 402.4 & 402.6 & 402.5 \\
\hline $2: 48: \odot \odot$ & 168 & 563.8 & 573.3 & 568.5 & 448.0 & 366.8 & 407.4 & 399.7 & 400.1 & 399.9 \\
\hline $2: 49: \odot \odot$ & 169 & 559.7 & 569.2 & 564.4 & 444.4 & 365.5 & 405.0 & 397.2 & 397.8 & 397.5 \\
\hline $2: 5 \odot: \odot \odot$ & 170 & 555.8 & 565.1 & 560.4 & 443.5 & 363.2 & 403.3 & 394.5 & 395.1 & 394.8 \\
\hline $2: 51: 00$ & 171 & 551.9 & 561.0 & 556.4 & 441.5 & 364.1 & 402.8 & 393.3 & 392.7 & 393.0 \\
\hline $2: 52: \odot \odot$ & 172 & 548.1 & 556.9 & 552.5 & 439.7 & 364.3 & 402.0 & 390.7 & $390 . \odot$ & 390.4 \\
\hline
\end{tabular}




\begin{tabular}{|c|c|c|c|c|c|c|c|c|c|c|}
\hline 2:53:๑๑ & 173 & 544.1 & 553.3 & 548.7 & 437.4 & 356.9 & 397.1 & 387.0 & 387.5 & 387.2 \\
\hline $2: 54: \odot \odot$ & 174 & 540.3 & 549.5 & 544.9 & 435.0 & 365.9 & 400.5 & 384.4 & 385.0 & 384.7 \\
\hline $2: 55: \odot \odot$ & 175 & 536.6 & 545.7 & 541.2 & 432.7 & 359.6 & 396.1 & 381.9 & 382.8 & 382.4 \\
\hline $2: 56: \odot \odot$ & 176 & 533.1 & 542.0 & 537.6 & 433.2 & 360.0 & 396.6 & 380.7 & 381.4 & 381.0 \\
\hline $2: 57: \odot \odot$ & 177 & 529.9 & 538.3 & 534.1 & 431.8 & 354.2 & 393.0 & 378.0 & 378.9 & 378.4 \\
\hline $2: 58: 0 \odot$ & 178 & 526.2 & 534.4 & 530.3 & 427.8 & 353.1 & 390.5 & 375.6 & 376.3 & 376.0 \\
\hline $2: 59: \odot \odot$ & 179 & 522.7 & 531.9 & 527.3 & 423.7 & 350.8 & 387.2 & 373.5 & 374.2 & 373.8 \\
\hline 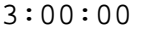 & 180 & 520.5 & 527.3 & 523.9 & 424.4 & 348.3 & 386.3 & 371.1 & 370.9 & 371.0 \\
\hline $3: 01: 00$ & 181 & 516.6 & 523.9 & 520.3 & 420.6 & 346.6 & 383.6 & 369.0 & 369.7 & 369.3 \\
\hline $3: \odot 2: \odot \odot$ & 182 & 512.8 & 520.9 & 516.8 & 420.6 & 341.2 & 380.9 & 366.6 & 367.2 & 366.9 \\
\hline $3: \odot 3: \odot \odot$ & 183 & 509.6 & 518.1 & 513.8 & 417.6 & 342.1 & 379.9 & 364.8 & 364.8 & 364.8 \\
\hline $3: \odot 4: \odot \odot$ & 184 & 506.8 & 514.7 & 510.7 & 416.3 & 343.6 & 379.9 & 361.6 & 362.7 & 362.1 \\
\hline $3: \odot 5: \odot \odot$ & 185 & 503.2 & 510.3 & 506.8 & 413.8 & 339.4 & 376.6 & 360.3 & 360.3 & 360.3 \\
\hline $3: \odot 6: \odot \odot$ & 186 & 500.4 & 507.6 & 504.0 & 411.3 & 336.9 & 374.1 & 357.8 & 356.7 & 357.3 \\
\hline $3: \odot 7: \odot \odot$ & 187 & 497.7 & 504.1 & 500.9 & 411.3 & 337.1 & 374.2 & 355.6 & 355.6 & 355.6 \\
\hline 3:०৪:๑९ & 188 & 493.9 & 501.3 & 497.6 & 406.6 & 332.6 & 369.6 & 352.9 & 352.9 & 352.9 \\
\hline $3: \odot 9: \odot \odot$ & 189 & 491.2 & 498.1 & 494.6 & 406.4 & 332.6 & 369.5 & 351.7 & 351.7 & 351.7 \\
\hline $3: 10: 00$ & 190 & 488.4 & 495.5 & 492.0 & 405.7 & 333.7 & 369.7 & 350.1 & 349.2 & 349.6 \\
\hline $3: 11: \odot \odot$ & 191 & 485.7 & 491.8 & 488.7 & 403.2 & 332.6 & 367.9 & 346.8 & 346.8 & 346.8 \\
\hline $3: 12: 0 \odot$ & 192 & 483.2 & 488.9 & 486.1 & 402.6 & 328.8 & 365.7 & 344.3 & 344.5 & 344.4 \\
\hline $3: 13: \odot \odot$ & 193 & 479.4 & 485.5 & 482.4 & 399.4 & 325.0 & 362.2 & 343.2 & 343.2 & 343.2 \\
\hline $3: 14: 0 \odot$ & 194 & 476.5 & 482.9 & 479.7 & 399.6 & 326.3 & 362.9 & 342.1 & 341.1 & 341.6 \\
\hline $3: 15: \odot \odot$ & 195 & 473.8 & 479.9 & 476.9 & 397.0 & 325.8 & 361.4 & 339.4 & 338.7 & 339.1 \\
\hline $3: 16: \odot \odot$ & 196 & 471.2 & 477.0 & 474.1 & 398.8 & 323.8 & 361.3 & 338.2 & 336.6 & 337.4 \\
\hline $3: 17: \odot \odot$ & 197 & 468.7 & 474.3 & 471.5 & 397.8 & 320.2 & 359.0 & 336.7 & 335.3 & 336.0 \\
\hline $3: 18: \odot \odot$ & 198 & 466.2 & 472.1 & 469.1 & 394.5 & 318.9 & 356.7 & 334.0 & 333.0 & 333.5 \\
\hline $3: 19: \odot \odot$ & 199 & 463.7 & 469.1 & 466.4 & 392.2 & 318.2 & 355.2 & 332.6 & 331.9 & 332.2 \\
\hline $3: 20: \odot \odot$ & 200 & 461.3 & 466.6 & 463.9 & 387.5 & 323.2 & 355.4 & 331.0 & 329.7 & 330.4 \\
\hline $3: 21: \odot \odot$ & 201 & 458.5 & 463.0 & 460.7 & 388.0 & 317.5 & 352.8 & 328.6 & 327.4 & 328.0 \\
\hline $3: 22: 0 \odot$ & 202 & 455.9 & 460.6 & 458.2 & 387.9 & 318.7 & 353.3 & 327.6 & 326.3 & 326.9 \\
\hline $3: 23: \odot \odot$ & 203 & 453.4 & 458.1 & 455.7 & 385.9 & 318.6 & 352.2 & 325.0 & 325.2 & 325.1 \\
\hline $3: 24: 0 \odot$ & 204 & 450.9 & 455.4 & 453.1 & 383.0 & 316.0 & 349.5 & 323.8 & 323.1 & 323.4 \\
\hline $3: 25: \odot \odot$ & 205 & 448.3 & 452.9 & 450.6 & 381.7 & 310.6 & 346.2 & 322.7 & 320.7 & 321.7 \\
\hline $3: 26: \odot \odot$ & 206 & 445.3 & 450.4 & 447.9 & 381.2 & 310.6 & 345.9 & 321.3 & 319.5 & 320.4 \\
\hline $3: 27: \odot \odot$ & 207 & 443.7 & 447.7 & 445.7 & 379.9 & 311.9 & 345.9 & 318.7 & 318.4 & 318.6 \\
\hline $3: 28: \odot \odot$ & 208 & 441.1 & 445.0 & 443.0 & 379.8 & 306.3 & 343.0 & 316.2 & 316.9 & 316.6 \\
\hline $3: 29: 0 \odot$ & 209 & 438.3 & 442.5 & .4 & 378.3 & 305.8 & 342.1 & 314.6 & 314.1 & 314.3 \\
\hline $3: 30: \odot \odot$ & 210 & 435.9 & 440.0 & 438.0 & 374.2 & 306.3 & 340.3 & 313.3 & 312.8 & 313.1 \\
\hline $3: 31: 0 \odot$ & 211 & 433.5 & 437.8 & 435.7 & 375.1 & 305.8 & 340.4 & 312.3 & 311.5 & 311.9 \\
\hline $3: 32: 0 \odot$ & 212 & 431.3 & 436.3 & 433.8 & 373.5 & 303.4 & 338.5 & 310.6 & 310.1 & 310.4 \\
\hline $3: 33: \odot \odot$ & 213 & 428.9 & 433.8 & 431.3 & 373.1 & 306.3 & 339.7 & 309.6 & 308.8 & 309.2 \\
\hline $3: 34: \odot \odot$ & 214 & 426.3 & 431.4 & 428.9 & 372.9 & 304.9 & 338.9 & 307.8 & 306.5 & 307.1 \\
\hline $3: 35: \odot \odot$ & 215 & 424.0 & 429.2 & 426.6 & 371.3 & 307.4 & 339.4 & 306.5 & 305.2 & 305.9 \\
\hline $3: 36: \odot \odot$ & 216 & 422.7 & 426.4 & 424.6 & 369.7 & 304.7 & 337.2 & 305.4 & 304.2 & 304.8 \\
\hline $3: 37: 0 \odot$ & 217 & 420.5 & 424.4 & 422.4 & 367.0 & 297.1 & 332.1 & 304.3 & 303.1 & 303.7 \\
\hline $3: 38: 00$ & 218 & 418.3 & 422.1 & 420.2 & 365.5 & 295.2 & 330.4 & 302.0 & 301.8 & 301.9 \\
\hline $3: 39: 0 \odot$ & 219 & 416.1 & 419.8 & 417.9 & 367.7 & 295.7 & 331.7 & 299.8 & 299.7 & 299.8 \\
\hline $3: 40: \odot \odot$ & 220 & 413.9 & 417.5 & 415.7 & 366.6 & 296.4 & 331.5 & 298.4 & 298.6 & 298.5 \\
\hline $3: 41: 00$ & 221 & 411.6 & 415.2 & 413.4 & 360.5 & 293.9 & 327.2 & 298.4 & 295.9 & 297.1 \\
\hline $3: 42: \odot \odot$ & 222 & 409.4 & 412.9 & 411.2 & 360.1 & 288.1 & 324.1 & 297.3 & 294.8 & 296.1 \\
\hline $3: 43: \odot \odot$ & 223 & $408 . \odot$ & 410.7 & 409.4 & 361.8 & 291.7 & 326.8 & 296.1 & 293.5 & 294.8 \\
\hline $3: 44: 0 \odot$ & 224 & 405.3 & 409.7 & 407.5 & 361.6 & 293.5 & 327.6 & 295.0 & 292.5 & 293.7 \\
\hline $3: 45: \odot \odot$ & 225 & 403.1 & 407.1 & 405.1 & 360.0 & 293.9 & 326.9 & 292.8 & 291.4 & 292.1 \\
\hline $3: 46: \odot \odot$ & 226 & 402.0 & 405.7 & 403.9 & 360.9 & 290.3 & 325.6 & 291.7 & 290.3 & 291.0 \\
\hline $3: 47: \odot \odot$ & 227 & 399.4 & 403.4 & 401.4 & 359.1 & 289.9 & 324.5 & 290.3 & 289.2 & 289.8 \\
\hline $3: 48: \odot \odot$ & 228 & 398.2 & 401.2 & 399.7 & 360.3 & 287.1 & 323.7 & 288.1 & 288.1 & 288.1 \\
\hline $3: 49: \odot \odot$ & 229 & 395.6 & 399.9 & 397.8 & 356.2 & 288.7 & 322.4 & 286.7 & 287.1 & 286.9 \\
\hline $3: 50: 00$ & 230 & 394.4 & 397.4 & 395.9 & 355.6 & 289.8 & 322.7 & 285.6 & $286 . \odot$ & 285.8 \\
\hline $3: 51: 0 \odot$ & 231 & 391.9 & 396.1 & 394.0 & 354.4 & 289.6 & 322.0 & 284.5 & 284.9 & 284.7 \\
\hline $3: 52: \odot \odot$ & 232 & 390.7 & 393.8 & 392.3 & 354.4 & 286.2 & 320.3 & 283.5 & 283.8 & 283.6 \\
\hline $3: 53: \odot \odot$ & 233 & 388.3 & 392.5 & 390.4 & 352.0 & 291.7 & 321.9 & 282.2 & 283.8 & 283.0 \\
\hline $3: 54: \odot \odot$ & 234 & 387.0 & 390.4 & 388.7 & 352.0 & 286.9 & 319.5 & 280.9 & 282.6 & 281.8 \\
\hline $3: 55: 00$ & 235 & 384.7 & 388.2 & 386.4 & 350.4 & 282.6 & 316.5 & 279.9 & 281.5 & 280.7 \\
\hline $3: 56: \odot \odot$ & 236 & 383.5 & 387.1 & 385.3 & 349.3 & 285.1 & 317.2 & 279.9 & 280.4 & 280.1 \\
\hline
\end{tabular}




\begin{tabular}{|c|c|c|c|c|c|c|c|c|c|c|}
\hline $3: 57: \odot \odot$ & 237 & 380.9 & 384.8 & 382.8 & 350.2 & 279.3 & 314.8 & 278.8 & 279.3 & 279.1 \\
\hline $3: 58: \odot \odot$ & 238 & 379.5 & 383.5 & 381.5 & 348.3 & 285.1 & 316.7 & 278.8 & 278.2 & 278.5 \\
\hline $3: 59: \odot \odot$ & 239 & 377.3 & 381.3 & 379.3 & 347.0 & 281.1 & 314.1 & 277.7 & 275.7 & 276.7 \\
\hline $4: \odot \odot: \odot \odot$ & 240 & 376.2 & 379.1 & 377.6 & 347.9 & 279.0 & 313.4 & 276.4 & 275.7 & 276.1 \\
\hline $4: \odot 1: \odot \odot$ & 241 & 374.0 & 377.8 & 375.9 & 345.9 & 279.0 & 312.4 & 276.4 & 274.6 & 275.5 \\
\hline $4: \odot 2: \odot \odot$ & 242 & 372.7 & 375.6 & 374.2 & 343.0 & 272.1 & 307.6 & 275.0 & 273.6 & 274.3 \\
\hline 4:०3:๑९ & 243 & 371.5 & 374.6 & 373.0 & 341.4 & 276.1 & 308.8 & 275.0 & 272.3 & 273.7 \\
\hline $4: \odot 4: \odot \odot$ & 244 & 369.4 & 372.3 & 370.9 & 342.3 & 276.1 & 309.2 & 273.7 & 271.2 & 272.5 \\
\hline 4:०5:०७ & 245 & 368.1 & 371.1 & 369.6 & 342.1 & 276.1 & 309.1 & 272.7 & 270.1 & 271.4 \\
\hline $4: \odot 6: \odot \odot$ & 246 & 365.9 & 368.8 & 367.4 & 340.3 & 271.4 & 305.9 & 271.4 & 268.0 & 269.7 \\
\hline $4: \odot 7: \odot \odot$ & 247 & 364.8 & 367.7 & 366.3 & 340.0 & 271.4 & 305.7 & 270.3 & 268.0 & 269.2 \\
\hline $4: \odot 8: \odot \odot$ & 248 & 362.8 & 366.6 & 364.7 & 336.6 & 275.2 & 305.9 & 269.2 & 266.7 & 268.0 \\
\hline $4: \odot 9: \odot \odot$ & 249 & 361.5 & 364.1 & 362.8 & 337.6 & 272.1 & $3 \odot 4.9$ & 268.0 & 265.6 & 266.8 \\
\hline $4: 10: 0 \odot$ & 250 & 360.2 & 362.9 & 361.6 & 337.3 & 270.7 & 304.0 & 266.9 & 264.6 & 265.7 \\
\hline $4: 11: \odot \odot$ & 251 & 358.1 & 360.8 & 359.5 & 335.7 & 268.5 & 302.1 & 265.6 & 263.5 & 264.6 \\
\hline $4: 12: \odot \odot$ & 252 & 357.0 & 359.7 & 358.4 & 335.7 & 267.6 & 301.6 & 264.6 & 262.0 & 263.3 \\
\hline $4: 13: \odot \odot$ & 253 & 354.7 & 357.4 & 356.1 & 334.0 & 270.0 & 302.0 & 263.5 & 261.0 & 262.2 \\
\hline $4: 14: 0 \odot$ & 254 & 353.5 & 356.4 & 354.9 & 335.1 & 266.7 & 300.9 & 263.5 & 259.9 & 261.7 \\
\hline $4: 15: \odot \odot$ & 255 & 352.4 & 354.2 & 353.3 & 331.9 & 267.8 & 299.8 & 261.3 & 259.9 & 260.6 \\
\hline $4: 16: \odot \odot$ & 256 & 351.0 & 353.1 & 352.1 & 331.9 & 264.4 & 298.1 & 261.3 & 258.6 & 260.0 \\
\hline $4: 17: \odot \odot$ & 257 & 349.9 & 352.0 & 351.0 & 329.4 & 265.5 & 297.4 & 260.2 & 257.5 & 258.9 \\
\hline 4:18:๑९ & 258 & 347.5 & 350.8 & 349.1 & 329.4 & 263.8 & 296.6 & 259.2 & 257.5 & 258.4 \\
\hline $4: 19: \odot \odot$ & 259 & 346.2 & 348.6 & 347.4 & 326.7 & 263.3 & 295.0 & 258.1 & 255.2 & 256.6 \\
\hline $4: 20: \odot \odot$ & 260 & 345.2 & 347.5 & 346.3 & 326.7 & 264.7 & 295.7 & 257.0 & 254.1 & 255.6 \\
\hline $4: 21: 00$ & 261 & 344.1 & 346.3 & 345.2 & 327.6 & 263.3 & 295.4 & 255.7 & 254.1 & 254.9 \\
\hline $4: 22: \odot \odot$ & 262 & 342.8 & 344.1 & 343.4 & 327.6 & 266.4 & 297.0 & 254.7 & 253.0 & 253.9 \\
\hline $4: 23: \odot \odot$ & 263 & 340.3 & 343.1 & 341.7 & 325.6 & 260.8 & 293.2 & 254.7 & 251.8 & 253.2 \\
\hline $4: 24: \odot \odot$ & 264 & 339.3 & 342.0 & 340.6 & 324.5 & 259.9 & 292.2 & 253.6 & 251.8 & 252.7 \\
\hline $4: 25: \odot \odot$ & 265 & 338.2 & 340.9 & 339.5 & 321.4 & 256.3 & 288.9 & 252.5 & 250.7 & 251.6 \\
\hline $4: 26: \odot \odot$ & 266 & 337.1 & 338.4 & 337.7 & 323.1 & 256.3 & 289.7 & 251.4 & 249.6 & 250.5 \\
\hline $4: 27: \odot \odot$ & 267 & 336.0 & 337.3 & 336.6 & 323.1 & 255.0 & 289.0 & 250.3 & 249.6 & 250.0 \\
\hline $4: 28: \odot \odot$ & 268 & 334.5 & 336.3 & 335.4 & 320.9 & 255.4 & 288.1 & 249.3 & 248.4 & 248.8 \\
\hline $4: 29: 0 \odot$ & 269 & 332.2 & 335.1 & 333.6 & 321.3 & 259.0 & 290.1 & 248.2 & 247.3 & 247.7 \\
\hline $4: 3 \odot: \odot \odot$ & 270 & 331.0 & 334.0 & 332.5 & 320.7 & 258.1 & 289.4 & 248.2 & 246.2 & 247.2 \\
\hline $4: 31: \odot \odot$ & 271 & 330.0 & 331.8 & 330.9 & 319.1 & 256.8 & 288.0 & 247.1 & 246.2 & 246.7 \\
\hline $4: 32: 0 \odot$ & 272 & 328.6 & 330.6 & 329.6 & 319.1 & 258.6 & 288.9 & 246.0 & 245.1 & 245.6 \\
\hline $4: 33: \odot \odot$ & 273 & 327.5 & 329.3 & 328.4 & 317.7 & 256.6 & 287.2 & 246.0 & 243.7 & 244.9 \\
\hline $4: 34: \odot \odot$ & 274 & 326.4 & 328.2 & 327.3 & 317.7 & 252.5 & 285.1 & 244.8 & 242.4 & 243.6 \\
\hline $4: 35: \odot \odot$ & 275 & 324.2 & 327.0 & 325.6 & 316.0 & 256.8 & 286.4 & 243.7 & 241.3 & 242.5 \\
\hline $4: 36: \odot \odot$ & 276 & 323.2 & 325.9 & 324.5 & 317.1 & 256.6 & 286.9 & 242.6 & 241.3 & 242.0 \\
\hline $4: 37: \odot \odot$ & 277 & 322.0 & 324.8 & 323.4 & 314.8 & 257.0 & 285.9 & 241.5 & 240.3 & 240.9 \\
\hline $4: 38: \odot \odot$ & 278 & 320.6 & 323.7 & 322.2 & 315.7 & 254.7 & 285.2 & 241.5 & 240.3 & 240.9 \\
\hline $4: 39: \odot \odot$ & 279 & 319.5 & 322.7 & 321.1 & 315.0 & 255.7 & 285.4 & 240.4 & 240.3 & 240.4 \\
\hline $4: 40: \odot \odot$ & 280 & 318.4 & 321.6 & 320.0 & 315.0 & 253.6 & 284.3 & 240.4 & 239.2 & 239.8 \\
\hline $4: 41: \odot \odot$ & 281 & 317.3 & 319.4 & 318.4 & 313.0 & 254.7 & 283.8 & 240.4 & 239.2 & 239.8 \\
\hline $4: 42: \odot \odot$ & 282 & 316.2 & 318.3 & 317.3 & 314.6 & 254.7 & 284.6 & 240.4 & 239.2 & 239.8 \\
\hline $4: 43: 00$ & 283 & 315.1 & 318.3 & 316.7 & 313.2 & 252.0 & 282.6 & 239.4 & 239.2 & 239.3 \\
\hline $4: 44: \odot \odot$ & 284 & 313.8 & 317.1 & 315.5 & 312.4 & 251.2 & 281.8 & 238.3 & 239.2 & 238.7 \\
\hline $4: 45: \odot \odot$ & 285 & 312.7 & 314.9 & 313.8 & 312.8 & 252.3 & 282.6 & 237.2 & 238.1 & 237.7 \\
\hline $4: 46: \odot \odot$ & 286 & 311.5 & 314.9 & 313.2 & 312.4 & 252.3 & 282.4 & 237.2 & 238.1 & 237.7 \\
\hline $4: 47: \odot \odot$ & 287 & 310.4 & 313.6 & 312.0 & 311.4 & 253.2 & 282.3 & 237.2 & 238.1 & 237.7 \\
\hline $4: 48: \odot \odot$ & 288 & 310.4 & 312.5 & 311.5 & 311.9 & 251.6 & 281.8 & 236.1 & 237.0 & 236.6 \\
\hline $4: 49: \odot \odot$ & 289 & 309.2 & 311.4 & 310.3 & 311.9 & 251.4 & 281.7 & 236.1 & 237.0 & 236.6 \\
\hline $4: 5 \odot: \odot \odot$ & 290 & 308.2 & 310.3 & 309.2 & 309.6 & 252.7 & 281.1 & 234.9 & 237.0 & 235.9 \\
\hline $4: 51: \odot \odot$ & 291 & 307.1 & 309.1 & 308.1 & 309.0 & 254.1 & 281.6 & 234.9 & 235.9 & 235.4 \\
\hline $4: 52: \odot \odot$ & 292 & 305.8 & 307.9 & 306.9 & 308.8 & 251.4 & 280.1 & 233.8 & 235.9 & 234.9 \\
\hline $4: 53: \odot \odot$ & 293 & 304.8 & 306.7 & 305.8 & 309.9 & 248.9 & 279.4 & 233.8 & 234.9 & 234.3 \\
\hline $4: 54: \odot \odot$ & 294 & 303.6 & 305.7 & 304.6 & 309.9 & 249.4 & 279.7 & 232.7 & 234.9 & 233.8 \\
\hline $4: 55: \odot \odot$ & 295 & 302.5 & $3 \odot 4.5$ & 303.5 & 308.5 & 250.7 & 279.6 & 231.6 & 234.9 & 233.2 \\
\hline $4: 56: 0 \odot$ & 296 & 301.2 & 303.4 & 302.3 & 308.1 & 248.0 & 278.1 & 231.6 & 233.8 & 232.7 \\
\hline $4: 57: \odot \odot$ & 297 & $30 \odot .1$ & 302.4 & 301.2 & 307.0 & 246.7 & 276.9 & 230.5 & 233.8 & 232.2 \\
\hline $4: 58: \odot \odot$ & 298 & 299.1 & 301.3 & 300.2 & 307.0 & 246.2 & 276.6 & 230.5 & 232.7 & 231.6 \\
\hline $4: 59: \odot \odot$ & 299 & 299.1 & 301.3 & 300.2 & 307.0 & 246.2 & 276.6 & 230.5 & 232.7 & 231.6 \\
\hline $5: \odot \odot: \odot \odot$ & 300 & 297.9 & 300.2 & 299.0 & 305.8 & 247.3 & 276.5 & 229.5 & 231.6 & 230.5 \\
\hline
\end{tabular}




\begin{tabular}{|c|c|c|c|c|c|c|c|c|c|c|}
\hline $5: \odot 1: \odot \odot$ & 301 & 296.8 & 299.0 & 297.9 & 305.8 & 246.2 & 276.0 & 229.5 & 231.6 & 230.5 \\
\hline $5: \odot 2: \odot \odot$ & 302 & 295.6 & 297.9 & 296.8 & 304.7 & 242.1 & 273.4 & 228.4 & 231.6 & 230.0 \\
\hline $5: \odot 3: \odot \odot$ & 303 & 294.3 & 296.9 & 295.6 & 303.3 & 246.2 & 274.7 & 227.3 & 230.4 & 228.8 \\
\hline $5: \odot 4: \odot \odot$ & 304 & 293.2 & 295.8 & 294.5 & 303.3 & 244.8 & 274.0 & 227.3 & 229.3 & 228.3 \\
\hline $5: \odot 5: \odot \odot$ & 305 & 293.2 & 294.7 & 294.0 & 301.3 & 244.6 & 272.9 & 225.9 & 228.2 & 227.0 \\
\hline $5: \odot 6: \odot \odot$ & 306 & 292.1 & 293.7 & 292.9 & 301.3 & 243.9 & 272.6 & 225.9 & 226.9 & 226.4 \\
\hline $5: \odot 7: \odot \odot$ & 307 & 291.0 & 293.7 & 292.3 & $30 \odot .7$ & 246.7 & 273.7 & 224.6 & 225.9 & 225.2 \\
\hline 5:०८:๑९ & 308 & 289.9 & 292.3 & 291.1 & 295.7 & 241.3 & 268.5 & 223.5 & 224.8 & 224.2 \\
\hline $5: \odot 9: \odot \odot$ & 309 & 288.9 & 291.2 & 290.0 & 297.1 & 239.2 & 268.2 & 222.3 & 223.7 & 223.0 \\
\hline $5: 10: \odot \odot$ & 310 & 287.8 & 289.9 & 288.9 & 297.9 & 243.7 & 270.8 & 221.2 & 222.6 & 221.9 \\
\hline $5: 11: 00$ & 311 & 286.8 & 288.7 & 287.7 & 296.8 & 239.4 & 268.1 & 220.1 & 221.4 & 220.7 \\
\hline $5: 12: 0 \odot$ & 312 & 285.7 & 288.7 & 287.2 & 298.0 & 241.0 & 269.5 & 220.1 & 221.4 & 220.7 \\
\hline $5: 13: \odot \odot$ & 313 & 285.7 & 287.5 & 286.6 & 296.4 & 239.7 & 268.1 & 218.8 & 220.3 & 219.6 \\
\hline $5: 14: 0 \odot$ & 314 & 284.6 & 286.4 & 285.5 & 295.7 & 239.9 & 267.8 & 218.8 & 220.3 & 219.6 \\
\hline 5:15:๑๑ & 315 & 283.5 & 285.4 & 284.4 & 294.6 & 238.1 & 266.4 & 218.8 & 220.3 & 219.6 \\
\hline $5: 16: \odot \odot$ & 316 & 282.3 & 284.1 & 283.2 & 294.8 & 237.9 & 266.4 & 218.8 & 220.3 & 219.6 \\
\hline $5: 17: \odot \odot$ & 317 & 281.3 & 284.1 & 282.7 & 296.2 & 238.8 & 267.5 & 218.8 & 220.3 & 219.6 \\
\hline $5: 18: 00$ & 318 & 281.3 & 283.1 & 282.2 & 296.2 & 238.1 & 267.2 & 218.8 & 219.2 & 219.0 \\
\hline $5: 19: \odot \odot$ & 319 & 280.1 & $282 . \odot$ & 281.0 & 297.7 & 234.9 & 266.3 & 217.8 & 219.2 & 218.5 \\
\hline $5: 20: \odot \odot$ & 320 & 278.9 & 280.9 & 279.9 & 293.0 & 239.2 & 266.1 & 217.8 & 219.2 & 218.5 \\
\hline $5: 21: \odot \odot$ & 321 & 278.9 & 280.9 & 279.9 & 294.1 & 238.3 & 266.2 & 216.7 & 219.2 & 217.9 \\
\hline $5: 22: \odot \odot$ & 322 & 277.7 & 279.7 & 278.7 & 292.6 & 235.9 & 264.3 & 216.7 & 218.1 & 217.4 \\
\hline $5: 23: 00$ & 323 & 276.7 & 278.7 & 277.7 & 292.5 & 235.2 & 263.8 & 215.6 & 218.1 & 216.9 \\
\hline $5: 24: \odot \odot$ & 324 & 275.6 & 277.6 & 276.6 & 292.5 & 237.9 & 265.2 & 215.6 & 218.1 & 216.9 \\
\hline $5: 25: 00$ & 325 & 275.6 & 276.6 & 276.1 & 292.3 & 241.0 & 266.6 & 214.5 & 216.9 & 215.7 \\
\hline $5: 26: \odot \odot$ & 326 & 274.4 & 276.6 & 275.5 & 291.2 & 236.8 & 264.0 & 214.5 & 216.9 & 215.7 \\
\hline $5: 27: \odot \odot$ & 327 & 273.3 & 275.5 & 274.4 & 290.7 & 233.6 & 262.1 & 214.5 & 215.8 & 215.2 \\
\hline $5: 28: \odot \odot$ & 328 & 272.2 & 274.5 & 273.3 & 289.9 & 233.8 & 261.9 & 213.4 & 215.8 & 214.6 \\
\hline $5: 29: \odot \odot$ & 329 & 272.2 & 273.5 & 272.8 & 289.9 & 233.8 & 261.9 & 213.4 & 215.8 & 214.6 \\
\hline $5: 30: \odot \odot$ & 330 & 270.9 & 273.5 & 272.2 & 289.4 & 232.3 & 260.9 & 213.4 & 215.8 & 214.6 \\
\hline $5: 31: 00$ & 331 & 270.9 & 272.5 & 271.7 & 289.4 & 232.7 & 261.1 & 212.2 & 214.7 & 213.4 \\
\hline $5: 32: \odot \odot$ & 332 & 269.8 & 271.4 & 270.6 & 287.6 & 235.2 & 261.4 & 212.2 & 214.7 & 213.4 \\
\hline $5: 33: \odot \odot$ & 333 & 268.7 & 270.4 & 269.5 & 288.3 & 231.3 & 259.8 & 212.2 & 214.7 & 213.4 \\
\hline $5: 34: \odot \odot$ & 334 & 268.7 & 270.4 & 269.5 & 287.4 & 234.7 & 261.1 & 212.2 & 214.7 & 213.4 \\
\hline $5: 35: \odot \odot$ & 335 & 267.6 & 269.4 & 268.5 & 287.6 & 231.6 & 259.6 & 211.1 & 213.4 & 212.3 \\
\hline $5: 36: \odot \odot$ & 336 & 266.5 & 268.4 & 267.4 & 284.0 & 231.1 & 257.5 & 210.0 & 212.4 & 211.2 \\
\hline $5: 37: \odot \odot$ & 337 & 265.3 & 267.1 & 266.2 & 282.0 & 232.5 & 257.3 & 208.9 & 211.3 & 210.1 \\
\hline $5: 38: \odot \odot$ & 338 & 265.3 & 267.1 & 266.2 & 283.5 & 229.3 & 256.4 & 207.9 & 210.0 & 208.9 \\
\hline $5: 39: \odot \odot$ & 339 & 264.2 & 266.1 & 265.2 & 283.3 & 228.7 & 256.0 & 206.8 & 208.9 & 207.9 \\
\hline $5: 4 \odot: \odot \odot$ & 340 & 263.0 & 264.9 & 264.0 & 282.0 & 226.9 & 254.5 & 205.7 & 207.9 & 206.8 \\
\hline $5: 41: \odot \odot$ & 341 & 263.0 & 264.9 & 264.0 & 279.9 & 226.4 & 253.1 & 205.7 & 207.9 & 206.8 \\
\hline $5: 42: \odot \odot$ & 342 & 262.0 & 263.8 & 262.9 & 279.5 & 230.9 & 255.2 & 205.7 & 206.4 & 206.1 \\
\hline $5: 43: \odot \odot$ & 343 & 260.9 & 262.6 & 261.8 & 279.7 & 227.8 & 253.8 & 204.6 & 206.4 & 205.5 \\
\hline $5: 44: 0 \odot$ & 344 & 260.9 & 262.6 & 261.8 & 277.7 & 222.4 & 250.1 & 204.6 & 205.3 & 205.0 \\
\hline $5: 45: \odot \odot$ & 345 & 259.9 & 261.6 & 260.7 & 279.0 & 224.8 & 251.9 & 204.6 & 204.3 & 204.4 \\
\hline $5: 46: \odot \odot$ & 346 & 258.6 & 260.5 & 259.6 & 276.8 & 222.6 & 249.7 & 203.5 & 204.3 & 203.9 \\
\hline $5: 47: \odot \odot$ & 347 & 258.6 & 259.4 & 259.0 & 276.3 & 221.2 & 248.7 & 203.5 & 203.2 & 203.4 \\
\hline $5: 48: \odot \odot$ & 348 & 257.6 & 259.4 & 258.5 & 277.3 & 224.6 & 251.0 & 202.5 & 203.2 & 202.8 \\
\hline $5: 49: \odot \odot$ & 349 & 256.6 & 258.3 & 257.4 & 276.3 & 222.4 & 249.4 & 202.5 & 202.1 & 202.3 \\
\hline $5: 5 \odot: \odot \odot$ & 350 & 256.6 & 257.3 & 256.9 & 274.5 & 221.5 & 248.0 & 202.5 & 202.1 & 202.3 \\
\hline $5: 51: \odot \odot$ & 351 & 255.5 & 257.3 & 256.4 & 275.0 & 221.7 & 248.4 & 201.4 & 202.1 & 201.7 \\
\hline $5: 52: 0 \odot$ & 352 & 254.4 & 256.1 & 255.3 & 275.0 & 222.8 & 248.9 & 201.4 & 200.8 & 201.1 \\
\hline $5: 53: \odot \odot$ & 353 & 254.4 & 255.1 & 254.8 & 275.0 & 220.6 & 247.8 & 201.4 & 200.8 & 201.1 \\
\hline $5: 54: \odot \odot$ & 354 & 253.3 & 255.1 & 254.2 & 274.3 & 222.8 & 248.5 & 200.3 & 199.8 & $20 \odot . \odot$ \\
\hline $5: 55: 0 \odot$ & 355 & 252.2 & 253.9 & 253.0 & 272.1 & 221.0 & 246.6 & 200.3 & 199.8 & 200.0 \\
\hline $5: 56: \odot \odot$ & 356 & 252.2 & 253.9 & 253.0 & 274.1 & 219.6 & 246.8 & 199.2 & 198.7 & 199.0 \\
\hline $5: 57: \odot \odot$ & 357 & 251.1 & 252.9 & 252.0 & 272.7 & 219.6 & 246.1 & 199.2 & 198.7 & 199.0 \\
\hline $5: 58: \odot \odot$ & 358 & 251.1 & 251.8 & 251.4 & 271.4 & 217.4 & 244.4 & 198.1 & 197.6 & 197.9 \\
\hline $5: 59: \odot \odot$ & 359 & $250 . \odot$ & 251.8 & 250.9 & 270.1 & 219.6 & 244.9 & 198.1 & 197.6 & 197.9 \\
\hline $6: 0 \odot: ० \odot$ & 360 & 248.8 & 250.8 & 249.8 & 270.1 & 216.1 & 243.1 & 198.1 & 196.5 & 197.3 \\
\hline $6: \odot 1: \odot \odot$ & 361 & 248.8 & 249.7 & 249.2 & 268.5 & 214.3 & 241.4 & 197.1 & 196.5 & 196.8 \\
\hline $6: \odot 2: \odot \odot$ & 362 & 247.6 & 249.7 & 248.7 & 268.7 & 218.8 & 243.8 & 197.1 & 195.4 & 196.3 \\
\hline $6: \odot 3: \odot \odot$ & 363 & 247.6 & 248.6 & 248.1 & 268.9 & 220.1 & 244.5 & 197.1 & 195.4 & 196.3 \\
\hline $6: \odot 4: \odot \odot$ & 364 & 246.5 & 247.5 & 247.0 & 267.8 & 216.9 & 242.3 & 196.0 & 195.4 & 195.7 \\
\hline
\end{tabular}




\begin{tabular}{|c|c|c|c|c|c|c|c|c|c|c|}
\hline $6: \odot 5: \odot \odot$ & 365 & 246.5 & 247.5 & 247.0 & 269.1 & 217.9 & 243.5 & 196.0 & 195.4 & 195.7 \\
\hline $6: \odot 6: \odot \odot$ & 366 & 245.3 & 246.4 & 245.9 & 267.3 & 217.6 & 242.4 & 196.0 & 195.4 & 195.7 \\
\hline $6: \odot 7: \odot \odot$ & 367 & 244.2 & 246.4 & 245.3 & 268.3 & 214.5 & 241.4 & 194.9 & 195.4 & 195.2 \\
\hline 6:๑৪:๑๑ & 368 & 244.2 & 245.4 & 244.8 & 265.1 & 213.6 & 239.4 & 194.9 & 194.2 & 194.5 \\
\hline $6: \odot 9: \odot \odot$ & 369 & 243.1 & 244.2 & 243.6 & 266.7 & 216.0 & 241.3 & 193.6 & 194.2 & 193.9 \\
\hline $6: 1 \odot: \odot \odot$ & 370 & 243.1 & 244.2 & 243.6 & 266.5 & 213.4 & 240.0 & 193.6 & 193.1 & 193.4 \\
\hline $6: 11: 0 \odot$ & 371 & 242.0 & 243.2 & 242.6 & 265.1 & 212.9 & 239.0 & 193.6 & 193.1 & 193.4 \\
\hline $6: 12: \odot \odot$ & 372 & 242.0 & 243.2 & 242.6 & 265.6 & 211.8 & 238.7 & 193.6 & 193.1 & 193.4 \\
\hline $6: 13: \odot \odot$ & 373 & 240.8 & 242.1 & 241.5 & 263.1 & 214.0 & 238.6 & 192.6 & 192.0 & 192.3 \\
\hline $6: 14: \odot \odot$ & 374 & 239.8 & 242.1 & 241.0 & 263.1 & 213.6 & 238.4 & 192.6 & 192.0 & 192.3 \\
\hline $6: 15: 0 \odot$ & 375 & 239.8 & 241.0 & 240.4 & 263.3 & 211.3 & 237.3 & 191.3 & 190.8 & 191.0 \\
\hline $6: 16: \odot \odot$ & 376 & 238.6 & 240.0 & 239.3 & 263.3 & 212.5 & 237.9 & 191.3 & 190.8 & 191.0 \\
\hline $6: 17: \odot \odot$ & 377 & 238.6 & $240 . \odot$ & 239.3 & 262.9 & 213.1 & 238.0 & 190.2 & 190.8 & 190.5 \\
\hline $6: 18: 0 \odot$ & 378 & 237.6 & 239.0 & 238.3 & 261.1 & 210.2 & 235.7 & 190.2 & 189.7 & 190.0 \\
\hline $6: 19: \odot \odot$ & 379 & 237.6 & 239.0 & 238.3 & 262.2 & 210.6 & 236.4 & 190.2 & 188.6 & 189.4 \\
\hline $6: 2 \odot: \odot \odot$ & 380 & 236.4 & 237.8 & 237.1 & 259.5 & 211.5 & 235.5 & 189.1 & 188.6 & 188.9 \\
\hline $6: 21: 0 \odot$ & 381 & 236.4 & 237.8 & 237.1 & 259.5 & 211.3 & 235.4 & 189.1 & 187.5 & 188.3 \\
\hline $6: 22: 0 \odot$ & 382 & 235.4 & 236.8 & 236.1 & 260.2 & 209.7 & 235.0 & 189.1 & 187.5 & 188.3 \\
\hline $6: 23: \odot \odot$ & 383 & 235.4 & 235.8 & 235.6 & 259.2 & 209.7 & 234.4 & 188.1 & 187.5 & 187.8 \\
\hline $6: 24: \odot \odot$ & 384 & 234.2 & 235.8 & 235.0 & 258.8 & 211.3 & 235.0 & 188.1 & 187.5 & 187.8 \\
\hline $6: 25: \odot \odot$ & 385 & 234.2 & 234.7 & 234.5 & 259.9 & 208.4 & 234.1 & 188.1 & 187.5 & 187.8 \\
\hline $6: 26: \odot \odot$ & 386 & 233.2 & 234.7 & 233.9 & 258.8 & 208.0 & 233.4 & 187.0 & 186.4 & 186.7 \\
\hline $6: 27: 0 \odot$ & 387 & 232.0 & 233.7 & 232.9 & 257.0 & 207.9 & 232.4 & 187.0 & 186.4 & 186.7 \\
\hline $6: 28: \odot \odot$ & 388 & 232.0 & 233.7 & 232.9 & 258.1 & 206.4 & 232.3 & 187.0 & 185.4 & 186.2 \\
\hline $6: 29: 0 \odot$ & 389 & 230.9 & 232.7 & 231.8 & 258.1 & 209.7 & 233.9 & 185.9 & 185.4 & 185.6 \\
\hline $6: 3 \odot: \odot \odot$ & 390 & 230.9 & 232.7 & 231.8 & 257.9 & 209.8 & 233.9 & 185.9 & 185.4 & 185.6 \\
\hline $6: 31: \odot \odot$ & 391 & 229.8 & 231.6 & 230.7 & 256.1 & 208.2 & 232.2 & 185.9 & 184.3 & 185.1 \\
\hline $6: 32: \odot \odot$ & 392 & 229.8 & 230.6 & 230.2 & 256.1 & 209.1 & 232.6 & 185.9 & 184.3 & 185.1 \\
\hline $6: 33: \odot \odot$ & 393 & 228.8 & 230.6 & 229.7 & 255.0 & 208.2 & 231.6 & 184.8 & 184.3 & 184.6 \\
\hline $6: 34: \odot \odot$ & 394 & 228.8 & 229.6 & 229.2 & 254.7 & 207.1 & 230.9 & 184.8 & 184.3 & 184.6 \\
\hline $6: 35: \odot \odot$ & 395 & 227.7 & 229.6 & 228.6 & 253.2 & 206.4 & 229.8 & 184.8 & 184.3 & 184.6 \\
\hline $6: 36: \odot \odot$ & 396 & 227.7 & 228.5 & 228.1 & 254.7 & 203.7 & 229.2 & 183.7 & 183.2 & 183.5 \\
\hline $6: 37: \odot \odot$ & 397 & 226.5 & 228.5 & 227.5 & 252.9 & 205.7 & 229.3 & 183.7 & 183.2 & 183.5 \\
\hline $6: 38: \odot \odot$ & 398 & 226.5 & 227.5 & 227.0 & 253.0 & 207.9 & 230.5 & 183.7 & 182.1 & 182.9 \\
\hline $6: 39: \odot \odot$ & 399 & 226.5 & 227.5 & 227.0 & 253.0 & 207.3 & 230.2 & 182.7 & 182.1 & 182.4 \\
\hline $6: 4 \odot: \odot \odot$ & 400 & 225.4 & 226.4 & 225.9 & 254.1 & 204.6 & 229.4 & 182.7 & 182.1 & 182.4 \\
\hline $6: 41: \odot \odot$ & 401 & 224.4 & 226.4 & 225.4 & 251.2 & 203.2 & 227.2 & 181.6 & 182.1 & 181.9 \\
\hline $6: 42: \odot \odot$ & 402 & 224.4 & 225.4 & 224.9 & 251.2 & 204.4 & 227.8 & 181.6 & 180.9 & 181.2 \\
\hline $6: 43: \odot \odot$ & 403 & 224.4 & 225.4 & 224.9 & 251.1 & 204.3 & 227.7 & 181.6 & 180.9 & 181.2 \\
\hline $6: 44: \odot \odot$ & 404 & 223.3 & 224.3 & 223.8 & 249.6 & 203.2 & 226.4 & 181.6 & 180.9 & 181.2 \\
\hline $6: 45: \odot \odot$ & 405 & 223.3 & 224.3 & 223.8 & 249.6 & 205.7 & 227.7 & 180.5 & 180.9 & 180.7 \\
\hline $6: 46: \odot \odot$ & 406 & 222.3 & 223.3 & 222.8 & 249.6 & 205.9 & 227.8 & 180.5 & 179.8 & 180.1 \\
\hline $6: 47: \odot \odot$ & 407 & 222.3 & 223.3 & 222.8 & 250.2 & 202.1 & 226.1 & 180.5 & 179.8 & 180.1 \\
\hline $6: 48: 0 \odot$ & 408 & 221.1 & 222.2 & 221.7 & 248.5 & 201.7 & 225.1 & 180.5 & 179.8 & 180.1 \\
\hline $6: 49: \odot \odot$ & 409 & 221.1 & 222.2 & 221.7 & 249.6 & 199.2 & 224.4 & 179.4 & 178.7 & 179.1 \\
\hline $6: 5 \odot: \odot \odot$ & 410 & 220.0 & 221.2 & 220.6 & 249.8 & 202.8 & 226.3 & 179.4 & 178.7 & 179.1 \\
\hline $6: 51: \odot \odot$ & 411 & 220.0 & 221.2 & 220.6 & 248.4 & 204.3 & 226.3 & 178.3 & 178.7 & 178.5 \\
\hline $6: 52: \odot \odot$ & 412 & 219.0 & 220.2 & 219.6 & 247.3 & 200.8 & 224.1 & 178.3 & 177.6 & 178.0 \\
\hline $6: 53: 00$ & 413 & 219.0 & 220.2 & 219.6 & 246.0 & 201.2 & 223.6 & 178.3 & 177.6 & 178.0 \\
\hline $6: 54: \odot \odot$ & 414 & 217.8 & 219.2 & 218.5 & 247.1 & 200.8 & 224.0 & 178.3 & 177.6 & 178.0 \\
\hline $6: 55: \odot \odot$ & 415 & 217.8 & 219.2 & 218.5 & 245.5 & 201.2 & 223.3 & 178.3 & 177.6 & 178.0 \\
\hline $6: 56: \odot \odot$ & 416 & 217.8 & 218.2 & 218.0 & 245.5 & 199.9 & 222.7 & 177.3 & 176.5 & 176.9 \\
\hline $6: 57: \odot \odot$ & 417 & 216.6 & 218.2 & 217.4 & 245.5 & 201.2 & 223.3 & 177.3 & 176.5 & 176.9 \\
\hline $6: 58: \odot \odot$ & 418 & 216.6 & 218.2 & 217.4 & 243.3 & 202.1 & 222.7 & 177.3 & 176.5 & 176.9 \\
\hline $6: 59: \odot \odot$ & 419 & 215.6 & 217.1 & 216.3 & 244.6 & 200.7 & 222.6 & 177.3 & 175.5 & 176.4 \\
\hline $7: \odot \odot: \odot \odot$ & 420 & 215.6 & 217.1 & 216.3 & 243.9 & 198.9 & 221.4 & 176.2 & 175.5 & 175.8 \\
\hline $7: \odot 1: \odot \odot$ & 421 & 215.6 & 216.1 & 215.8 & 242.8 & 198.9 & 220.8 & 176.2 & 175.5 & 175.8 \\
\hline $7: \odot 2: \odot \odot$ & 422 & 214.5 & 216.1 & 215.3 & 244.0 & 198.3 & 221.2 & 176.2 & 175.5 & 175.8 \\
\hline $7: \odot 3: \odot \odot$ & 423 & 214.5 & 215.0 & 214.8 & 242.8 & 196.7 & 219.7 & 174.9 & 174.2 & 174.6 \\
\hline $7: 04: 0 \odot$ & 424 & 213.5 & 215.0 & 214.3 & 242.8 & 195.6 & 219.2 & 174.9 & 174.2 & 174.6 \\
\hline $7: \odot 5: \odot \odot$ & 425 & 213.5 & 214.0 & 213.8 & 241.2 & 199.8 & 220.5 & 174.9 & 174.2 & 174.6 \\
\hline $7: \odot 6: \odot \odot$ & 426 & 212.4 & 214.0 & 213.2 & 242.2 & 197.8 & 220.0 & 173.7 & 173.1 & 173.4 \\
\hline $7: \odot 7: \odot \odot$ & 427 & 212.4 & 214.0 & 213.2 & 241.0 & 195.4 & 218.2 & 173.7 & 173.1 & 173.4 \\
\hline 7:๑৪:๑९ & 428 & 211.2 & 212.9 & 212.1 & 240.3 & 197.2 & 218.8 & 173.7 & 173.1 & 173.4 \\
\hline
\end{tabular}




\begin{tabular}{|c|c|c|c|c|c|c|c|c|c|c|}
\hline $7: \odot 9: \odot \odot$ & 429 & 211.2 & 212.9 & 212.1 & 240.3 & 195.6 & 217.9 & 172.6 & 172.0 & 172.3 \\
\hline $7: 10: \odot \odot$ & 430 & 210.2 & 211.9 & 211.0 & 241.0 & 194.2 & 217.6 & 172.6 & 172.0 & 172.3 \\
\hline $7: 11: \odot \odot$ & 431 & 210.2 & 211.9 & 211.0 & 239.7 & 192.4 & 216.1 & 172.6 & 172.0 & 172.3 \\
\hline $7: 12: \odot \odot$ & 432 & 210.2 & 210.8 & 210.5 & 238.5 & 194.4 & 216.4 & 171.5 & 172.0 & 171.8 \\
\hline $7: 13: \odot \odot$ & 433 & 209.1 & 210.8 & 209.9 & 239.9 & 195.8 & 217.9 & 171.5 & 171.0 & 171.2 \\
\hline $7: 14: \odot \odot$ & 434 & 209.1 & 210.8 & 209.9 & 238.8 & 193.3 & 216.1 & 171.5 & 171.0 & 171.2 \\
\hline $7: 15: \odot \odot$ & 435 & $208 . \odot$ & 209.7 & 208.9 & 238.8 & 195.1 & 217.0 & 170.4 & 171.0 & 170.7 \\
\hline $7: 16: \odot \odot$ & 436 & 208.0 & 209.7 & 208.9 & 237.6 & 193.6 & 215.6 & 170.4 & 171.0 & 170.7 \\
\hline $7: 17: \odot \odot$ & 437 & 208.0 & 208.7 & 208.4 & 237.6 & 194.0 & 215.8 & 170.4 & 171.0 & 170.7 \\
\hline $7: 18: \odot \odot$ & 438 & 207.0 & 208.7 & 207.8 & 236.1 & 193.1 & 214.6 & 170.4 & 169.9 & 170.2 \\
\hline $7: 19: \odot \odot$ & 439 & 207.0 & 207.6 & 207.3 & 236.1 & 191.8 & 214.0 & 169.3 & 169.9 & 169.6 \\
\hline $7: 2 \odot: \odot \odot$ & 440 & 205.8 & 207.6 & 206.7 & 237.4 & 190.4 & 213.9 & 169.3 & 168.8 & 169.1 \\
\hline $7: 21: \odot \odot$ & 441 & 205.8 & 207.6 & 206.7 & 236.5 & 190.2 & 213.4 & 169.3 & 168.8 & 169.1 \\
\hline $7: 22: \odot \odot$ & 442 & 205.8 & 206.6 & 206.2 & 234.5 & 193.5 & 214.0 & 168.1 & 167.7 & 167.9 \\
\hline $7: 23: \odot \odot$ & 443 & 204.7 & 206.6 & 205.6 & 234.9 & 194.2 & 214.5 & 168.1 & 167.7 & 167.9 \\
\hline $7: 24: \odot \odot$ & 444 & 204.7 & 205.6 & 205.1 & 233.8 & 191.8 & 212.8 & 168.1 & 167.7 & 167.9 \\
\hline $7: 25: \odot \odot$ & 445 & 204.7 & 205.6 & 205.1 & 234.0 & $190 . \odot$ & 212.0 & 168.1 & 167.7 & 167.9 \\
\hline $7: 26: \odot \odot$ & 446 & 203.6 & 204.5 & 204.1 & 233.4 & 191.5 & 212.5 & 168.1 & 167.7 & 167.9 \\
\hline $7: 27: \odot \odot$ & 447 & 203.6 & 204.5 & 204.1 & 233.4 & 188.6 & 211.0 & 168.1 & 167.7 & 167.9 \\
\hline $7: 28: \odot \odot$ & 448 & 202.6 & 204.5 & 203.5 & 231.8 & 192.2 & 212.0 & 167.0 & 167.7 & 167.4 \\
\hline $7: 29: \odot \odot$ & 449 & 202.6 & 203.5 & 203.0 & 231.6 & 189.3 & 210.5 & 167.0 & 167.7 & 167.4 \\
\hline $7: 3 \odot: \odot \odot$ & 450 & 202.6 & 203.5 & 203.0 & 232.7 & 190.4 & 211.6 & 167.0 & 166.6 & 166.8 \\
\hline $7: 31: \odot \odot$ & 451 & 201.5 & 203.5 & 202.5 & 232.7 & 193.6 & 213.2 & 167.0 & 166.6 & 166.8 \\
\hline $7: 32: \odot \odot$ & 452 & 201.5 & 202.5 & 202.0 & 231.6 & 190.0 & 210.8 & 167.0 & 166.6 & 166.8 \\
\hline $7: 33: \odot \odot$ & 453 & 201.5 & 202.5 & 202.0 & 231.6 & 190.6 & 211.1 & 165.7 & 165.6 & 165.7 \\
\hline $7: 34: \odot \odot$ & 454 & 200.3 & 201.5 & 200.9 & 230.5 & 189.5 & 210.0 & 165.7 & 165.6 & 165.7 \\
\hline $7: 35: \odot \odot$ & 455 & 200.3 & 201.5 & 200.9 & 230.5 & 187.7 & 209.1 & 165.7 & 165.6 & 165.7 \\
\hline $7: 36: \odot \odot$ & 456 & 199.2 & 201.5 & 200.4 & 230.5 & 186.1 & 208.3 & 164.7 & 165.6 & 165.1 \\
\hline $7: 37: \odot \odot$ & 457 & 199.2 & 200.4 & 199.8 & 229.1 & 189.7 & 209.4 & 164.7 & 165.6 & 165.1 \\
\hline $7: 38: \odot \odot$ & 458 & 199.2 & 200.4 & 199.8 & 229.1 & 186.6 & 207.9 & 164.7 & 165.6 & 165.1 \\
\hline $7: 39: \odot \odot$ & 459 & 198.1 & 199.3 & 198.7 & 229.1 & 184.8 & 207.0 & 164.7 & 165.6 & 165.1 \\
\hline $7: 40: \odot \odot$ & 460 & 198.1 & 199.3 & 198.7 & 229.1 & 188.4 & 208.8 & 163.6 & 164.5 & 164.0 \\
\hline $7: 41: \odot \odot$ & 461 & 198.1 & 199.3 & 198.7 & 229.1 & 186.6 & 207.9 & 163.6 & 164.5 & 164.0 \\
\hline $7: 42: \odot \odot$ & 462 & 197.1 & 198.3 & 197.7 & 227.8 & 188.1 & 208.0 & 163.6 & 164.5 & 164.0 \\
\hline $7: 43: \odot \odot$ & 463 & 197.1 & 198.3 & 197.7 & 227.8 & 188.1 & 208.0 & 163.6 & 163.4 & 163.5 \\
\hline $7: 44: \odot \odot$ & 464 & 197.1 & 198.3 & 197.7 & 228.9 & 186.8 & 207.9 & 162.5 & 163.4 & 163.0 \\
\hline $7: 45: \odot \odot$ & 465 & 196.0 & 197.3 & 196.7 & 227.8 & 184.3 & 206.1 & 162.5 & 163.4 & 163.0 \\
\hline $7: 46: \odot \odot$ & 466 & $196 . \odot$ & 197.3 & 196.7 & 226.4 & 187.2 & 206.8 & 162.5 & 162.3 & 162.4 \\
\hline $7: 47: \odot \odot$ & 467 & $196 . \odot$ & 196.3 & 196.1 & 225.1 & 186.1 & 205.6 & 162.5 & 162.3 & 162.4 \\
\hline $7: 48: \odot \odot$ & 468 & 194.9 & 196.3 & 195.6 & 225.5 & 186.6 & 206.1 & 162.5 & 162.3 & 162.4 \\
\hline $7: 49: \odot \odot$ & 469 & 194.9 & 196.3 & 195.6 & 225.5 & 187.2 & 206.3 & 161.4 & 162.3 & 161.9 \\
\hline $7: 5 \odot: \odot \odot$ & 470 & 194.9 & 195.2 & 195.1 & 225.3 & 183.4 & 204.4 & 161.4 & 161.2 & 161.3 \\
\hline $7: 51: \odot \odot$ & 471 & 193.8 & 195.2 & 194.5 & 224.2 & 183.4 & 203.8 & 161.4 & 161.2 & 161.3 \\
\hline $7: 52: \odot \odot$ & 472 & 193.8 & 195.2 & 194.5 & 224.2 & 183.6 & 203.9 & 161.4 & 161.2 & 161.3 \\
\hline $7: 53: \odot \odot$ & 473 & 192.8 & 194.1 & 193.4 & 223.2 & 185.0 & 204.1 & 160.2 & 160.0 & 160.1 \\
\hline $7: 54: \odot \odot$ & 474 & 192.8 & 194.1 & 193.4 & 223.2 & 181.9 & 202.6 & 160.2 & $160 . \odot$ & 160.1 \\
\hline $7: 55: \odot \odot$ & 475 & 192.8 & 194.1 & 193.4 & 223.7 & 182.8 & 203.3 & 160.2 & 160.0 & 160.1 \\
\hline $7: 56: \odot \odot$ & 476 & 192.8 & 193.1 & 192.9 & 223.9 & 184.8 & 204.4 & 160.2 & 160.0 & 160.1 \\
\hline $7: 57: \odot \odot$ & 477 & 191.8 & 193.1 & 192.4 & 222.3 & 183.4 & 202.8 & 159.1 & $160 . \odot$ & 159.5 \\
\hline $7: 58: 0 \odot$ & 478 & 191.8 & 193.1 & 192.4 & 222.8 & 181.2 & 202.0 & 159.1 & 158.9 & 159.0 \\
\hline $7: 59: \odot \odot$ & 479 & 190.7 & 192.0 & 191.3 & 222.8 & 181.2 & 202.0 & 159.1 & 158.9 & 159.0 \\
\hline ৪:०९:๑९ & 480 & 190.7 & 192.0 & 191.3 & 221.4 & 182.3 & 201.8 & 159.1 & 158.9 & 159.0 \\
\hline ৪:०1:०९ & 481 & 190.7 & 192.0 & 191.3 & 223.0 & 181.0 & $202 . \odot$ & 159.1 & 158.9 & 159.0 \\
\hline ৪:०2:०๑ & 482 & 189.6 & 190.9 & 190.3 & 220.6 & 182.7 & 201.7 & 159.1 & 158.9 & 159.0 \\
\hline ৪:๑3:๑९ & 483 & 189.6 & 190.9 & 190.3 & 220.6 & 179.8 & 200.2 & 159.1 & 158.9 & 159.0 \\
\hline $8: \odot 4: \odot \odot$ & 484 & 189.6 & 190.9 & 190.3 & 219.4 & 179.4 & 199.4 & 158.0 & 158.9 & 158.5 \\
\hline $8: \odot 5: \odot \odot$ & 485 & 189.6 & 189.8 & 189.7 & 219.4 & 183.0 & 201.2 & 158.0 & 157.8 & 157.9 \\
\hline ৪:๑6:๑๑ & 486 & 188.6 & 189.8 & 189.2 & 219.9 & 181.4 & 200.7 & 158.0 & 157.8 & 157.9 \\
\hline ৪:๑7:๑९ & 487 & 188.6 & 189.8 & 189.2 & 221.2 & 180.0 & 200.6 & 156.9 & 157.8 & 157.4 \\
\hline ৪:๑৪:๑९ & 488 & 188.6 & 188.8 & 188.7 & 219.6 & 179.8 & 199.7 & 156.9 & 157.8 & 157.4 \\
\hline ৪:०९:๑७ & 489 & 187.4 & 188.8 & 188.1 & 219.6 & 178.5 & 199.0 & 156.9 & 156.7 & 156.8 \\
\hline $8: 10: \odot \odot$ & 490 & 187.4 & 188.8 & 188.1 & 218.3 & 177.1 & 197.7 & 156.9 & 156.7 & 156.8 \\
\hline $8: 11: \odot \odot$ & 491 & 187.4 & 187.8 & 187.6 & 219.4 & 179.6 & 199.5 & 156.9 & 156.7 & 156.8 \\
\hline $8: 12: \odot \odot$ & 492 & 186.3 & 187.8 & 187.0 & 217.9 & 178.7 & 198.3 & 155.8 & 156.7 & 156.3 \\
\hline
\end{tabular}




\begin{tabular}{|c|c|c|c|c|c|c|c|c|c|c|}
\hline 8:13:๑९ & 493 & 186.3 & 186.7 & 186.5 & 217.9 & 176.5 & 197.2 & 155.8 & 156.7 & 156.3 \\
\hline ৪:14:๑९ & 494 & 186.3 & 186.7 & 186.5 & 218.1 & 178.0 & 198.1 & 155.8 & 156.7 & 156.3 \\
\hline ৪:15:๑๑ & 495 & 185.2 & 186.7 & 186.0 & 216.9 & 179.2 & 198.1 & 155.8 & 155.7 & 155.8 \\
\hline ৪:16:๑९ & 496 & 185.2 & 186.7 & 186.0 & 216.9 & 176.7 & 196.8 & 155.8 & 155.7 & 155.8 \\
\hline $8: 17: \odot \odot$ & 497 & 185.2 & 185.7 & 185.5 & 216.9 & 176.2 & 196.5 & 154.8 & 155.7 & 155.2 \\
\hline ৪:18:०๑ & 498 & 184.2 & 185.7 & 185.0 & 216.9 & 179.6 & 198.2 & 154.8 & 155.7 & 155.2 \\
\hline $8: 19: \odot \odot$ & 499 & 184.2 & 185.7 & 185.0 & 216.9 & 178.3 & 197.6 & 154.8 & 154.4 & 154.6 \\
\hline $8: 20: \odot \odot$ & 500 & 184.2 & 184.7 & 184.4 & 216.9 & 176.9 & 196.9 & 154.8 & 154.4 & 154.6 \\
\hline $8: 21: 0 \odot$ & 501 & 184.2 & 184.7 & 184.4 & 215.8 & 176.7 & 196.3 & 154.8 & 154.4 & 154.6 \\
\hline $8: 22: \odot \odot$ & 502 & 183.2 & 184.7 & 183.9 & 217.2 & 177.8 & 197.5 & 154.8 & 154.4 & 154.6 \\
\hline $8: 23: \odot \odot$ & 503 & 183.2 & 183.7 & 183.4 & 216.3 & 176.4 & 196.3 & 153.7 & 154.4 & 154.0 \\
\hline $8: 24: \odot \odot$ & 504 & 183.2 & 183.7 & 183.4 & 214.0 & 178.3 & 196.2 & 153.7 & 154.4 & 154.0 \\
\hline $8: 25: 0 \odot$ & 505 & 182.0 & 183.7 & 182.8 & 214.0 & 178.3 & 196.2 & 153.7 & 154.4 & 154.0 \\
\hline $8: 26: \odot \odot$ & 506 & 182.0 & 182.6 & 182.3 & 214.0 & 176.5 & 195.3 & 153.7 & 154.4 & 154.0 \\
\hline ৪:27:๑९ & 507 & 182.0 & 182.6 & 182.3 & 214.0 & 175.5 & 194.7 & 153.7 & 154.4 & 154.0 \\
\hline $8: 28: \odot \odot$ & 508 & 181.0 & 182.6 & 181.8 & 214.0 & 175.5 & 194.7 & 153.7 & 153.3 & 153.5 \\
\hline ৪:29:๑९ & 509 & 181.0 & 182.6 & 181.8 & 214.0 & 176.9 & 195.4 & 152.6 & 153.3 & 153.0 \\
\hline $8: 30: \odot \odot$ & 510 & 181.0 & 181.6 & 181.3 & 211.1 & 175.5 & 193.3 & 152.6 & 153.3 & 153.0 \\
\hline $8: 31: \odot \odot$ & 511 & 181.0 & 181.6 & 181.3 & 211.1 & 175.6 & 193.4 & 152.6 & 152.2 & 152.4 \\
\hline $8: 32: \odot \odot$ & 512 & 180.0 & 181.6 & 180.8 & 211.1 & 175.1 & 193.1 & 152.6 & 152.2 & 152.4 \\
\hline $8: 33: 00$ & 513 & 180.0 & 180.6 & 180.3 & 212.4 & 174.7 & 193.6 & 152.6 & 152.2 & 152.4 \\
\hline ৪: $34: \odot \odot$ & 514 & 180.0 & 180.6 & 180.3 & 212.4 & 172.6 & 192.5 & 152.6 & 152.2 & 152.4 \\
\hline $8: 35: \odot \odot$ & 515 & 178.9 & 180.6 & 179.7 & 212.5 & 174.6 & 193.6 & 151.5 & 152.2 & 151.9 \\
\hline $8: 36: \odot \odot$ & 516 & 178.9 & 179.5 & 179.2 & 211.3 & 173.7 & 192.5 & 151.5 & 152.2 & 151.9 \\
\hline $8: 37: \odot \odot$ & 517 & 178.9 & 179.5 & 179.2 & 210.2 & 174.6 & 192.4 & 151.5 & 152.2 & 151.9 \\
\hline $8: 38: \odot \odot$ & 518 & 178.9 & 179.5 & 179.2 & 210.4 & 174.7 & 192.6 & 151.5 & 152.2 & 151.9 \\
\hline $8: 39: \odot \odot$ & 519 & 177.8 & 179.5 & 178.7 & 210.7 & 174.7 & 192.7 & 150.4 & 151.0 & 150.7 \\
\hline ৪: 4०:०७ & 520 & 177.8 & 178.5 & 178.2 & 209.5 & 173.3 & 191.4 & 150.4 & 151.0 & 150.7 \\
\hline $8: 41: 0 \odot$ & 521 & 177.8 & 178.5 & 178.2 & 209.5 & 174.6 & 192.0 & 150.4 & 151.0 & 150.7 \\
\hline $8: 42: \odot \odot$ & 522 & 176.8 & 178.5 & 177.7 & 209.5 & 172.2 & 190.9 & 150.4 & 151.0 & 150.7 \\
\hline $8: 43: \odot \odot$ & 523 & 176.8 & 177.5 & 177.1 & 208.2 & 172.4 & 190.3 & 150.4 & 151.0 & 150.7 \\
\hline $8: 44: \odot \odot$ & 524 & 176.8 & 177.5 & 177.1 & 208.4 & 172.4 & 190.4 & 149.4 & 149.9 & 149.6 \\
\hline $8: 45: \odot \odot$ & 525 & 176.8 & 177.5 & 177.1 & 208.4 & 171.9 & 190.1 & 149.4 & 149.9 & 149.6 \\
\hline $8: 46: \odot \odot$ & 526 & 175.7 & 177.5 & 176.6 & 208.4 & 172.6 & 190.5 & 149.4 & 149.9 & 149.6 \\
\hline $8: 47: \odot \odot$ & 527 & 175.7 & 176.5 & 176.1 & 207.3 & 174.2 & 190.8 & 149.4 & 149.9 & 149.6 \\
\hline $8: 48: \odot \odot$ & 528 & 175.7 & 176.5 & 176.1 & 207.3 & 171.3 & 189.3 & 148.3 & 149.9 & 149.1 \\
\hline $8: 49: \odot \odot$ & 529 & 175.7 & 176.5 & 176.1 & 207.3 & 170.4 & 188.9 & 148.3 & 149.9 & 149.1 \\
\hline $8: 5 \odot: \odot \odot$ & 530 & 174.6 & 176.5 & 175.5 & 207.3 & 170.4 & 188.9 & 148.3 & 149.9 & 149.1 \\
\hline $8: 51: \odot \odot$ & 531 & 174.6 & 175.4 & 175.0 & 206.2 & 170.4 & 188.3 & 148.3 & 148.8 & 148.6 \\
\hline $8: 52: \odot \odot$ & 532 & 174.6 & 175.4 & 175.0 & 206.2 & 170.8 & 188.5 & 148.3 & 148.8 & 148.6 \\
\hline $8: 53: \odot \odot$ & 533 & 174.6 & 175.4 & 175.0 & 206.2 & 170.8 & 188.5 & 148.3 & 148.8 & 148.6 \\
\hline $8: 54: \odot \odot$ & 534 & 173.6 & 174.4 & 174.0 & 207.3 & 171.7 & 189.5 & 148.3 & 148.8 & 148.6 \\
\hline $8: 55: \odot \odot$ & 535 & 173.6 & 174.4 & 174.0 & 206.2 & 168.1 & 187.2 & 148.3 & 148.8 & 148.6 \\
\hline $8: 56: \odot \odot$ & 536 & 173.6 & 174.4 & 174.0 & 204.6 & 171.3 & 188.0 & 147.2 & 147.7 & 147.5 \\
\hline $8: 57: \odot \odot$ & 537 & 173.6 & 174.4 & 174.0 & 204.6 & 172.2 & 188.4 & 147.2 & 147.7 & 147.5 \\
\hline $8: 58: \odot \odot$ & 538 & 172.5 & 173.4 & 173.0 & 204.6 & 169.7 & 187.2 & 147.2 & 147.7 & 147.5 \\
\hline $8: 59: \odot \odot$ & 539 & 172.5 & 173.4 & 173.0 & 204.6 & 167.5 & 186.1 & 147.2 & 147.7 & 147.5 \\
\hline ๑:०९:๑९ & 540 & 172.5 & 173.4 & 173.0 & 203.4 & 168.8 & 186.1 & 147.2 & 147.7 & 147.5 \\
\hline $9: \odot 1: \odot \odot$ & 541 & 172.5 & 173.4 & 173.0 & 202.1 & 168.6 & 185.4 & 146.1 & 146.7 & 146.4 \\
\hline $9: \odot 2: \odot \odot$ & 542 & 171.4 & 172.4 & 171.9 & 203.2 & 167.4 & 185.3 & 146.1 & 146.7 & 146.4 \\
\hline $9: \odot 3: \odot \odot$ & 543 & 171.4 & 172.4 & 171.9 & 203.2 & 163.6 & 183.4 & 146.1 & 146.7 & 146.4 \\
\hline $9: \odot 4: \odot \odot$ & 544 & 171.4 & 172.4 & 171.9 & 203.2 & 167.5 & 185.4 & 146.1 & 146.7 & 146.4 \\
\hline $9: \odot 5: \odot \odot$ & 545 & 171.4 & 172.4 & 171.9 & 203.2 & 169.0 & 186.1 & 146.1 & 146.7 & 146.4 \\
\hline 9:๑6:๑९ & 546 & 171.4 & 171.3 & 171.3 & 203.2 & 167.9 & 185.5 & 146.1 & 145.6 & 145.9 \\
\hline $9: \odot 7: \odot \odot$ & 547 & 170.2 & 171.3 & 170.7 & 203.2 & 167.9 & 185.5 & 146.1 & 145.6 & 145.9 \\
\hline $9: \odot 8: \odot \odot$ & 548 & 170.2 & 171.3 & 170.7 & 202.1 & 168.3 & 185.2 & 146.1 & 145.6 & 145.9 \\
\hline $9: \odot 9: \odot \odot$ & 549 & 170.2 & 171.3 & 170.7 & 202.1 & 168.3 & 185.2 & 145.0 & 145.6 & 145.3 \\
\hline $9: 10: \odot \odot$ & 550 & 170.2 & 170.3 & 170.2 & 201.6 & 168.8 & 185.2 & 145.0 & 145.6 & 145.3 \\
\hline $9: 11: \odot \odot$ & 551 & 169.1 & 170.3 & 169.7 & 201.6 & 167.5 & 184.6 & 145.0 & 145.6 & 145.3 \\
\hline $9: 12: \odot \odot$ & 552 & 169.1 & 170.3 & 169.7 & 200.1 & 166.8 & 183.5 & 145.0 & 145.6 & 145.3 \\
\hline $9: 13: \odot \odot$ & 553 & 169.1 & 170.3 & 169.7 & 200.1 & 167.7 & 183.9 & 145.0 & 144.5 & 144.8 \\
\hline $9: 14: \odot \odot$ & 554 & 169.1 & 169.3 & 169.2 & 199.6 & 167.7 & 183.7 & 145.0 & 144.5 & 144.8 \\
\hline $9: 15: \odot \odot$ & 555 & 169.1 & 169.3 & 169.2 & 198.5 & 165.9 & 182.2 & 145.0 & 144.5 & 144.8 \\
\hline $9: 16: \odot \odot$ & 556 & 168.0 & 169.3 & 168.6 & 198.7 & 167.4 & 183.0 & 144.0 & 144.5 & 144.2 \\
\hline
\end{tabular}




\begin{tabular}{|c|c|c|c|c|c|c|c|c|c|c|}
\hline $9: 17: \odot \odot$ & 557 & 168.0 & 169.3 & 168.6 & 199.9 & 167.2 & 183.6 & 144.0 & 144.5 & 144.2 \\
\hline 9:18:๑๑ & 558 & $168 . \odot$ & 168.2 & 168.1 & 198.9 & 165.7 & 182.3 & 144.0 & 144.5 & 144.2 \\
\hline $9: 19: \odot \odot$ & 559 & 168.0 & 168.2 & 168.1 & 198.7 & 166.8 & 182.8 & 144.0 & 144.5 & 144.2 \\
\hline $9: 2 \odot: \odot \odot$ & 560 & 167.0 & 168.2 & 167.6 & 197.6 & 165.9 & 181.8 & 142.9 & 144.5 & 143.7 \\
\hline $9: 21: \odot \odot$ & 561 & 167.0 & 168.2 & 167.6 & 199.0 & 164.7 & 181.9 & 142.9 & 143.4 & 143.2 \\
\hline $9: 22: \odot \odot$ & 562 & 167.0 & 167.2 & 167.1 & 197.2 & 164.8 & 181.0 & 142.9 & 143.4 & 143.2 \\
\hline $9: 23: \odot \odot$ & 563 & 167.0 & 167.2 & 167.1 & 197.2 & 166.3 & 181.8 & 142.9 & 143.4 & 143.2 \\
\hline $9: 24: \odot \odot$ & 564 & 165.9 & 167.2 & 166.6 & 198.3 & 166.8 & 182.6 & 142.9 & 143.4 & 143.2 \\
\hline $9: 25: \odot \odot$ & 565 & 165.9 & 167.2 & 166.6 & 197.2 & 163.8 & 180.5 & 142.9 & 143.4 & 143.2 \\
\hline $9: 26: \odot \odot$ & 566 & 165.9 & 166.1 & 166.0 & 197.2 & 163.8 & 180.5 & 142.9 & 143.4 & 143.2 \\
\hline $9: 27: \odot \odot$ & 567 & 165.9 & 166.1 & 166.0 & 196.7 & 166.8 & 181.8 & 141.8 & 142.3 & 142.1 \\
\hline $9: 28: \odot \odot$ & 568 & 165.9 & 166.1 & 166.0 & 196.7 & 162.1 & 179.4 & 141.8 & 142.3 & 142.1 \\
\hline $9: 29: \odot \odot$ & 569 & 164.8 & 166.1 & 165.4 & 196.9 & 165.7 & 181.3 & 141.8 & 142.3 & 142.1 \\
\hline $9: 3 \odot: \odot \odot$ & 570 & 164.8 & 166.1 & 165.4 & 195.8 & 163.2 & 179.5 & 141.8 & 142.3 & 142.1 \\
\hline $9: 31: \odot \odot$ & 571 & 164.8 & 165.1 & 164.9 & 195.8 & 164.8 & 180.3 & 141.8 & 142.3 & 142.1 \\
\hline $9: 32: 0 \odot$ & 572 & 164.8 & 165.1 & 164.9 & 195.8 & 164.3 & 180.1 & 141.8 & 142.3 & 142.1 \\
\hline $9: 33: \odot \odot$ & 573 & 164.8 & 165.1 & 164.9 & 195.8 & 163.2 & 179.5 & 141.8 & 142.3 & 142.1 \\
\hline $9: 34: \odot \odot$ & 574 & 163.8 & 165.1 & 164.4 & 194.5 & 163.0 & 178.8 & 141.8 & 142.3 & 142.1 \\
\hline $9: 35: \odot \odot$ & 575 & 163.8 & 164.0 & 163.9 & 194.5 & 163.9 & 179.2 & 141.8 & 141.3 & 141.5 \\
\hline $9: 36: \odot \odot$ & 576 & 163.8 & 164.0 & 163.9 & 194.5 & 162.7 & 178.6 & 140.7 & 141.3 & 141.0 \\
\hline $9: 37: 0 \odot$ & 577 & 163.8 & 164.0 & 163.9 & 195.4 & 163.2 & 179.3 & 140.7 & 141.3 & 141.0 \\
\hline $9: 38: \odot \odot$ & 578 & 162.7 & 164.0 & 163.4 & 193.8 & 162.1 & 178.0 & 140.7 & 141.3 & 141.0 \\
\hline $9: 39: \odot \odot$ & 579 & 162.7 & 163.0 & 162.9 & 193.8 & 160.5 & 177.2 & 140.7 & 141.3 & 141.0 \\
\hline $9: 4 \odot: \odot \odot$ & 580 & 162.7 & 163.0 & 162.9 & 193.8 & 160.7 & 177.3 & 140.7 & 140.2 & 140.5 \\
\hline $9: 41: \odot \odot$ & 581 & 162.7 & 163.0 & 162.9 & 192.6 & 162.1 & 177.4 & 140.7 & 140.2 & 140.5 \\
\hline $9: 42: \odot \odot$ & 582 & 162.7 & 163.0 & 162.9 & 192.6 & 160.9 & 176.7 & 140.7 & 140.2 & 140.5 \\
\hline $9: 43: \odot \odot$ & 583 & 161.6 & 163.0 & 162.3 & 192.6 & 159.4 & 176.0 & 140.7 & 140.2 & 140.5 \\
\hline $9: 44: \odot \odot$ & 584 & 161.6 & 162.0 & 161.8 & 191.3 & 158.9 & 175.1 & 139.5 & 140.2 & 139.8 \\
\hline $9: 45: \odot \odot$ & 585 & 161.6 & 162.0 & 161.8 & 192.6 & 159.6 & 176.1 & 139.5 & 139.1 & 139.3 \\
\hline $9: 46: \odot \odot$ & 586 & 161.6 & 162.0 & 161.8 & 192.6 & 161.1 & 176.8 & 139.5 & 139.1 & 39.3 \\
\hline $9: 47: \odot \odot$ & 587 & 161.6 & 162.0 & 161.8 & 192.6 & 161.1 & 176.8 & 139.5 & 139.1 & 9.3 \\
\hline $9: 48: \odot \odot$ & 588 & 160.5 & 162.0 & 161.2 & 192.6 & 159.4 & 176.0 & 139.5 & 139.1 & 39.3 \\
\hline $9: 49: \odot \odot$ & 589 & 160.5 & 160.9 & 160.7 & 190.9 & 160.0 & 175.5 & 139.5 & 139.1 & 139.3 \\
\hline $9: 50: \odot \odot$ & 590 & 160.5 & 160.9 & 160.7 & 190.9 & 160.0 & 175.5 & 138.4 & 139.1 & 138.7 \\
\hline 9: $51: \odot \odot$ & 591 & 160.5 & 160.9 & 160.7 & 190.9 & 160.9 & 175.9 & 138.4 & 139.1 & 138.7 \\
\hline $9: 52: \odot \odot$ & 592 & 160.5 & 160.9 & 160.7 & 189.9 & 160.5 & 175.2 & 138.4 & 139.1 & 138.7 \\
\hline $9: 53: \odot \odot$ & 593 & 159.5 & 159.9 & 159.7 & 189.9 & 158.4 & 174.1 & 138.4 & 139.1 & 138.7 \\
\hline $9: 54: \odot \odot$ & 594 & 159.5 & 159.9 & 159.7 & 190.9 & 160.5 & 175.7 & 138.4 & $138 . \odot$ & 138.2 \\
\hline $9: 55: \odot \odot$ & 595 & 159.5 & 159.9 & 159.7 & 189.9 & 160.5 & 175.2 & 138.4 & 138.0 & 138.2 \\
\hline $9: 56: \odot \odot$ & 596 & 159.5 & 159.9 & 159.7 & 189.9 & 158.5 & 174.2 & 138.4 & 138.0 & 138.2 \\
\hline $9: 57: \odot \odot$ & 597 & 159.5 & 159.9 & 159.7 & 188.6 & 158.5 & 173.6 & 138.4 & $138 . \odot$ & 138.2 \\
\hline $9: 58: 0 \odot$ & 598 & 158.4 & 158.9 & 158.6 & 190.0 & 157.1 & 173.6 & 137.1 & 136.9 & 137.0 \\
\hline $9: 59: \odot \odot$ & 599 & 158.4 & 158.9 & 158.6 & 188.8 & 157.3 & 173.0 & 137.1 & 136.9 & 137.0 \\
\hline 1०: $\odot \odot: \odot \odot$ & 600 & 158.4 & 158.9 & 158.6 & 189.1 & 160.0 & 174.6 & 7.1 & 136.9 & 7.0 \\
\hline $10: 01: 00$ & 601 & 158.4 & 158.9 & 158.6 & 187.9 & 157.5 & 172.7 & 137.1 & 136.9 & 137.0 \\
\hline $10: \odot 2: \odot \odot$ & 602 & 158.4 & 158.9 & 158.6 & 186.8 & 159.4 & 173.1 & 137.1 & 136.9 & 137.0 \\
\hline $10: 03: \odot \odot$ & 603 & 157.3 & 158.9 & 158.1 & 187.9 & 158.4 & 173.1 & 137.1 & 136.9 & 137.0 \\
\hline $10: 04: \odot \odot$ & 604 & 157.3 & 157.8 & 157.6 & 187.9 & 156.7 & 172.3 & 137.1 & 136.9 & 137.0 \\
\hline 1०: $\odot 5: \odot \odot$ & 605 & 157.3 & 157.8 & 157.6 & 187.9 & 159.4 & 173.7 & 137.1 & 136.9 & 137.0 \\
\hline $10: 06: 0 \odot$ & 606 & 157.3 & 157.8 & 157.6 & 186.6 & 157.6 & 172.1 & 137.1 & 136.9 & 137.0 \\
\hline $10: \odot 7: \odot \odot$ & $6 \odot 7$ & 157.3 & 157.8 & 157.6 & 186.6 & 158.7 & 172.7 & 137.1 & 136.9 & 137.0 \\
\hline 1०:०৪:०९ & 608 & 156.3 & 156.8 & 156.5 & 186.6 & 157.1 & 171.9 & 136.0 & 135.9 & 136.0 \\
\hline $10: \odot 9: \odot \odot$ & 609 & 156.3 & 156.8 & 156.5 & 185.4 & 156.4 & 170.9 & 136.0 & 135.9 & 136.0 \\
\hline $10: 10: 00$ & 610 & 156.3 & 156.8 & 156.5 & 186.4 & 157.8 & 172.1 & 136.0 & 135.9 & 136.0 \\
\hline $10: 11: \odot \odot$ & 611 & 156.3 & 156.8 & 156.5 & 185.4 & 157.8 & 171.6 & 136.0 & 135.9 & 136.0 \\
\hline $10: 12: \odot \odot$ & 612 & 156.3 & 156.8 & 156.5 & 184.3 & 155.3 & 169.8 & 136.0 & 135.9 & 136.0 \\
\hline $10: 13: \odot \odot$ & 613 & 155.2 & 155.8 & 155.5 & 185.9 & 155.1 & 170.5 & 136.0 & 135.9 & 136.0 \\
\hline $1 \odot: 14: \odot \odot$ & 614 & 155.2 & 155.8 & 155.5 & 184.1 & 156.7 & 170.4 & 135.0 & 134.8 & 134.9 \\
\hline $10: 15: \odot \odot$ & 615 & 155.2 & 155.8 & 155.5 & 184.1 & 154.6 & 169.3 & 135.0 & 134.8 & 134.9 \\
\hline $10: 16: \odot \odot$ & 616 & 155.2 & 155.8 & 155.5 & 184.1 & 152.2 & 168.2 & 135.0 & 134.8 & 134.9 \\
\hline $10: 17: \odot \odot$ & 617 & 155.2 & 155.8 & 155.5 & 184.1 & 155.8 & 170.0 & 135.0 & 134.8 & 134.9 \\
\hline $10: 18: \odot \odot$ & 618 & 154.1 & 154.8 & 154.5 & 184.1 & 155.7 & 169.9 & 135.0 & 134.8 & 134.9 \\
\hline $10: 19: \odot \odot$ & 619 & 154.1 & 154.8 & 154.5 & 184.3 & 155.5 & 169.9 & 135.0 & 134.8 & 134.9 \\
\hline $10: 20: \odot \odot$ & 620 & 154.1 & 154.8 & 154.5 & 184.3 & 154.0 & 169.2 & 135.0 & 134.8 & 134.9 \\
\hline
\end{tabular}




\begin{tabular}{|c|c|c|c|c|c|c|c|c|c|c|}
\hline $10: 21: 0 \odot$ & 621 & 154.1 & 154.8 & 154.5 & 182.8 & 153.5 & 168.2 & 133.9 & 134.8 & 134.3 \\
\hline $10: 22: \odot \odot$ & 622 & 154.1 & 154.8 & 154.5 & 182.7 & 154.0 & 168.4 & 133.9 & 134.8 & 134.3 \\
\hline $1 \odot: 23: \odot \odot$ & 623 & 154.1 & 154.8 & 154.5 & 181.6 & 155.5 & 168.5 & 133.9 & 134.8 & 134.3 \\
\hline $10: 24: 0 \odot$ & 624 & 153.1 & 153.8 & 153.4 & 182.8 & 155.5 & 169.2 & 133.9 & 134.8 & 134.3 \\
\hline $10: 25: \odot \odot$ & 625 & 153.1 & 153.8 & 153.4 & 182.8 & 154.4 & 168.6 & 133.9 & 134.8 & 134.3 \\
\hline $10: 26: \odot \odot$ & 626 & 153.1 & 153.8 & 153.4 & 182.8 & 157.3 & 170.1 & 133.9 & 134.8 & 134.3 \\
\hline$\odot: 27: \odot \odot$ & 627 & 153.1 & 153.8 & 153.4 & 182.8 & 155.8 & 169.3 & 133.9 & 134.8 & 134.3 \\
\hline $1 \odot: 28: \odot \odot$ & 628 & 153.1 & 153.8 & 153.4 & 182.8 & 155.8 & 169.3 & 133.9 & 134.8 & 134.3 \\
\hline $10: 29: 00$ & 629 & 153.1 & 153.8 & 153.4 & 182.8 & 157.3 & 170.1 & 133.9 & 134.8 & 134.3 \\
\hline $10: 30: \odot \odot$ & 630 & 152.1 & 152.8 & 152.4 & 181.8 & 155.8 & 168.8 & 133.9 & 134.8 & 134.3 \\
\hline $10: 31: 00$ & 631 & 152.1 & 152.8 & 152.4 & 181.8 & 157.3 & 169.5 & 133.9 & 134.8 & 134.3 \\
\hline $10: 32: 0 \odot$ & 632 & 152.1 & 152.8 & 152.4 & 181.8 & 155.8 & 168.8 & 133.9 & 134.8 & 134.3 \\
\hline $1 \odot: 33: \odot \odot$ & 633 & 152.1 & 152.8 & 152.4 & 181.8 & 155.8 & 168.8 & 133.9 & 134.8 & 134.3 \\
\hline $10: 34: 00$ & 634 & 152.1 & 152.8 & 152.4 & 181.8 & 156.0 & 168.9 & 133.9 & 134.8 & 134.3 \\
\hline $10: 35: \odot \odot$ & 635 & 152.1 & 152.8 & 152.4 & 181.8 & 154.6 & 168.2 & 133.9 & 134.8 & 134.3 \\
\hline $1 \odot: 36: \odot \odot$ & 636 & 152.1 & 152.8 & 152.4 & 180.5 & 156.2 & 168.4 & 133.9 & 134.8 & 134.3 \\
\hline $10: 37: 0 \odot$ & 637 & 151.0 & 151.7 & 151.4 & 181.6 & 156.2 & 168.9 & 133.9 & 134.8 & 134.3 \\
\hline $10: 38: \odot \odot$ & 638 & 151.0 & 151.7 & 151.4 & 181.6 & 155.7 & 168.6 & 133.9 & 134.8 & 134.3 \\
\hline $1 \odot: 39: \odot \odot$ & 639 & 151.0 & 151.7 & 151.4 & 180.1 & 154.8 & 167.5 & 133.9 & 134.8 & 134.3 \\
\hline $10: 40: 0 \odot$ & 640 & 151.0 & 151.7 & 151.4 & 181.8 & 156.4 & 169.1 & 133.9 & 134 & 134.3 \\
\hline $10: 41: \odot \odot$ & 641 & 151.0 & 151.7 & 151.4 & 180.5 & 155.3 & 167.9 & 133.9 & 134.8 & 134.3 \\
\hline $10: 42: 0 \odot$ & 642 & 151.0 & 151.7 & 151.4 & 180.5 & 154.6 & 167.5 & 133.9 & 133.7 & 133.8 \\
\hline $10: 43: \odot \odot$ & 643 & 151.0 & 151.7 & 151.4 & 180.5 & 154.6 & 167.5 & 133.9 & 133.7 & 133.8 \\
\hline $10: 44: \odot \odot$ & 644 & 149.9 & 150.7 & 150.3 & 180.5 & 154.0 & 167.3 & 133.9 & 133.7 & 133.8 \\
\hline $10: 45: \odot \odot$ & 645 & 149.9 & 150.7 & 150.3 & 179.4 & 154.0 & 166.7 & 133.9 & 133.7 & 133.8 \\
\hline $10: 46: \odot \odot$ & 646 & 149.9 & 150.7 & 150.3 & 179.4 & 154.6 & 167.0 & 133.9 & 133.7 & 133.8 \\
\hline $1 \odot: 47: \odot \odot$ & 647 & 149.9 & 150.7 & 150.3 & 179.4 & 155.8 & 167.6 & 133.9 & 133.7 & 133.8 \\
\hline $10: 48: 00$ & 648 & 149.9 & 150.7 & 150.3 & 178.3 & 155.8 & 167.1 & 133.9 & 133.7 & 133.8 \\
\hline $1 \odot: 49: \odot \odot$ & 649 & 149.9 & 150.7 & 150.3 & 178.3 & 154.8 & 166.6 & 133.9 & 133.7 & 133.8 \\
\hline $10: 50: \odot \odot$ & 650 & 149.9 & 150.7 & 150.3 & 178.3 & 152.4 & 165.4 & 133.9 & 133 & 133.8 \\
\hline $10: 51: \odot \odot$ & 651 & 148.9 & 149.7 & 149.3 & 178.3 & 153.7 & 166.0 & 133.9 & 133 & 133.8 \\
\hline $1 \odot: 52: \odot \odot$ & 652 & 148.9 & 149.7 & 149.3 & 178.3 & 154.8 & 166.6 & 133.9 & 133 & 133.8 \\
\hline $10: 53: \odot \odot$ & 653 & 148.9 & 149.7 & 149.3 & 177.1 & 154.2 & 165.7 & 133.9 & 133.7 & 133.8 \\
\hline $1 \odot: 54: \odot \odot$ & 654 & 148.9 & 149.7 & 149.3 & 178.2 & 153.7 & 165.9 & 133.9 & 133.7 & 133.8 \\
\hline $1 \odot: 55: \odot \odot$ & 655 & 148.9 & 149.7 & 149.3 & 177.1 & 153.7 & 165.4 & 133.9 & 133.7 & 133.8 \\
\hline $10: 56: \odot \odot$ & 656 & 148.9 & 149.7 & 149.3 & 177.1 & 155.1 & 166.1 & 133.9 & 132.6 & 133.3 \\
\hline $1 \odot: 57: \odot \odot$ & 657 & 148.9 & 148.6 & 148.8 & 175.8 & 155.5 & 165.7 & 133.9 & 132.6 & 133.3 \\
\hline $10: 58: \odot \odot$ & 658 & 147.9 & 148.6 & 148.3 & 175.8 & 154.0 & 164.9 & 133.9 & 132.6 & 133.3 \\
\hline $1 \odot: 59: \odot \odot$ & 659 & 147.9 & 148.6 & 148.3 & 175.8 & 154.2 & 165.0 & 132.6 & 132.6 & 132.6 \\
\hline $11: \odot \odot: \odot \odot$ & 660 & 147.9 & 148.6 & 148.3 & 175.8 & 153.1 & 164.5 & 132.6 & 132.6 & 132.6 \\
\hline $11: 01: 00$ & 661 & 147.9 & 148.6 & 148.3 & 175.8 & 153.1 & 164.5 & 132.6 & 132.6 & 132.6 \\
\hline $11: \odot 2: \odot \odot$ & 662 & 147.9 & 148.6 & 148.3 & 175.8 & 154.0 & 164.9 & 132.6 & 132.6 & 132.6 \\
\hline $11: 03: \odot \odot$ & 663 & 147.9 & 148.6 & 148.3 & 174.7 & 153.5 & 164.1 & 132.6 & 131.5 & 132.1 \\
\hline $11: \odot 4: \odot \odot$ & 664 & 147.9 & 147.6 & 147.7 & 176.0 & 153.1 & 164.6 & 132.6 & 131.5 & 132.1 \\
\hline $11: \odot 5: \odot \odot$ & 665 & 146.8 & 147.6 & 147.2 & 174.9 & 151.9 & 163.4 & 132.6 & 131.5 & 132.1 \\
\hline $11: \odot 6: \odot \odot$ & 666 & 146.8 & 147.6 & 147.2 & 174.9 & 151.9 & 163.4 & 132.6 & 131.5 & 132.1 \\
\hline $11: 07: 00$ & 667 & 146.8 & 147.6 & 147.2 & 174.9 & 151.9 & 163.4 & 132.6 & 131.5 & 132.1 \\
\hline $11: \odot 8: \odot \odot$ & 668 & 146.8 & 147.6 & 147.2 & 174.9 & 151.2 & 163.0 & 132.6 & 131.5 & 132.1 \\
\hline $11: \odot 9: \odot \odot$ & 669 & 146.8 & 147.6 & 147.2 & 174.9 & 153.5 & 164.2 & 132.6 & 131.5 & 132.1 \\
\hline $11: 10: \odot \odot$ & 670 & 146.8 & 147.6 & 147.2 & 174.9 & 150.8 & 162.9 & 132.6 & 131.5 & 132.1 \\
\hline $11: 11: \odot \odot$ & 671 & 146.8 & 147.6 & 147.2 & 173.5 & 153.0 & 163.2 & 132.6 & 131.5 & 132.1 \\
\hline $11: 12: \odot \odot$ & 672 & 146.8 & 146.5 & 146.6 & 173.5 & 151.9 & 162.7 & 131.5 & 131.5 & 131.5 \\
\hline $11: 13: \odot \odot$ & 673 & 145.8 & 146.5 & 146.1 & 173.5 & 150.3 & 161.9 & 131.5 & 131.5 & 131.5 \\
\hline $11: 14: \odot \odot$ & 674 & 145.8 & 146.5 & 146.1 & 173.5 & 152.6 & 163.0 & 131.5 & 130.5 & 131.0 \\
\hline $11: 15: \odot \odot$ & 675 & 145.8 & 146.5 & 146.1 & 173.5 & 151.0 & 162.2 & 131.5 & 130.5 & 131.0 \\
\hline $11: 16: \odot \odot$ & 676 & 145.8 & 146.5 & 146.1 & 173.5 & 150.8 & 162.1 & 131.5 & 130.5 & 131.0 \\
\hline $11: 17: \odot \odot$ & 677 & 145.8 & 146.5 & 146.1 & 173.5 & 150.8 & 162.1 & 131.5 & 130.5 & 131.0 \\
\hline $11: 18: \odot \odot$ & 678 & 145.8 & 146.5 & 146.1 & 172.4 & 151.9 & 162.1 & 131.5 & 130.5 & 131.0 \\
\hline $11: 19: \odot \odot$ & 679 & 145.8 & 145.5 & 145.6 & 172.4 & 150.8 & 161.6 & 131.5 & 130.5 & 131.0 \\
\hline $11: 20: 00$ & 680 & 145.8 & 145.5 & 145.6 & 172.4 & 150.8 & 161.6 & 130.5 & 130.5 & 130.5 \\
\hline $11: 21: \odot \odot$ & 681 & 144.7 & 145.5 & 145.1 & 172.4 & 149.7 & 161.1 & 130.5 & 130.5 & 130.5 \\
\hline $11: 22: \odot \odot$ & 682 & 144.7 & 145.5 & 145.1 & 172.4 & 150.3 & 161.3 & 130.5 & 130.5 & 130.5 \\
\hline $11: 23: \odot \odot$ & 683 & 144.7 & 145.5 & 145.1 & 172.4 & 150.4 & 161.4 & 130.5 & 130.5 & 130.5 \\
\hline $11: 24: \odot \odot$ & 684 & 144.7 & 145.5 & 145.1 & 171.3 & 150.4 & 160.9 & 130.5 & 130.5 & 130.5 \\
\hline
\end{tabular}




\begin{tabular}{|c|c|c|c|c|c|c|c|c|c|c|}
\hline $11: 25: \odot \odot$ & 685 & 144.7 & 145.5 & 145.1 & 171.3 & 152.1 & 161.7 & 130.5 & 130.5 & 130.5 \\
\hline $11: 26: \odot \odot$ & 686 & 144.7 & 145.5 & 145.1 & 171.3 & 150.3 & 160.8 & 130.5 & 129.4 & 129.9 \\
\hline $11: 27: \odot \odot$ & 687 & 144.7 & 144.5 & 144.6 & 170.1 & 150.3 & 160.2 & 130.5 & 129.4 & 129.9 \\
\hline $11: 28: 0 \odot$ & 688 & 143.7 & 144.5 & 144.1 & 171.1 & 150.8 & 161.0 & 130.5 & 129.4 & 129.9 \\
\hline $11: 29: \odot \odot$ & 689 & 143.7 & 144.5 & 144.1 & 171.1 & 148.6 & 159.9 & 130.5 & 129.4 & 129.9 \\
\hline $11: 30: 0 \odot$ & 690 & 143.7 & 144.5 & 144.1 & 171.1 & 150.8 & 161.0 & 130.5 & 129.4 & 129.9 \\
\hline $11: 31: 0 \odot$ & 691 & 143.7 & 144.5 & 144.1 & 170.1 & 150.8 & 160.4 & 130.5 & 129.4 & 129.9 \\
\hline $11: 32: \odot \odot$ & 692 & 143.7 & 144.5 & 144.1 & 170.1 & 148.1 & 159.1 & 130.5 & 129.4 & 129.9 \\
\hline $11: 33: 00$ & 693 & 143.7 & 144.5 & 144.1 & 170.1 & 147.9 & 159.0 & 130.5 & 129.4 & 129.9 \\
\hline $11: 34: \odot \odot$ & 694 & 143.7 & 143.5 & 143.6 & 171.1 & 147.0 & 159.1 & 129.4 & 129.4 & 129.4 \\
\hline $11: 35: 0 \odot$ & 695 & 143.7 & 143.5 & 143.6 & 169.3 & 150.3 & 159.8 & 129.4 & 129.4 & 129.4 \\
\hline $11: 36: 0 \odot$ & 696 & 143.7 & 143.5 & 143.6 & 169.3 & 150.3 & 159.8 & 129.4 & 129.4 & 129.4 \\
\hline $11: 37: \odot \odot$ & 697 & 142.7 & 143.5 & 143.1 & 169.3 & 147.9 & 158.6 & 129.4 & 129.4 & 129.4 \\
\hline $11: 38: 0 \odot$ & 698 & 142.7 & 143.5 & 143.1 & 168.1 & 145.8 & 156.9 & 129.4 & 129.4 & 129.4 \\
\hline $11: 39: \odot \odot$ & 699 & 142.7 & 143.5 & 143.1 & 169.2 & 148.5 & 158.8 & 129.4 & 128.3 & 128.8 \\
\hline $11: 4 \odot: \odot \odot$ & 700 & 142.7 & 143.5 & 143.1 & 169.2 & 146.7 & 157.9 & 129.4 & 128.3 & 128.8 \\
\hline $11: 41: 0 \odot$ & 701 & 142.7 & 143.5 & 143.1 & 168.1 & 148.5 & 158.3 & 129.4 & 128.3 & 128.8 \\
\hline $11: 42: \odot \odot$ & 702 & 142.7 & 142.4 & 142.5 & 168.1 & 147.4 & 157.7 & 129.4 & 128.3 & 128.8 \\
\hline $11: 43: \odot \odot$ & 703 & 142.7 & 142.4 & 142.5 & 168.1 & 148.5 & 158.3 & 129.4 & 128.3 & 128.8 \\
\hline $11: 44: \odot \odot$ & 704 & 142.7 & 2.4 & 2.5 & 168.1 & 149.0 & 158.5 & 129.4 & 128.3 & 128.8 \\
\hline $11: 45: \odot \odot$ & 705 & 141.6 & 142.4 & 142.0 & 168.1 & 147.6 & 157.8 & 129.4 & 128.3 & 128.8 \\
\hline $11: 46: \odot \odot$ & 706 & 141.6 & 142.4 & 142.0 & 167.0 & 146.3 & 156.7 & 129.4 & 128.3 & 128.8 \\
\hline $11: 47: \odot \odot$ & 707 & 141.6 & 142.4 & 142.0 & 166.8 & 148.3 & 157.6 & 129.4 & 128.3 & 128.8 \\
\hline $11: 48: \odot \odot$ & 708 & 141.6 & 142.4 & 142.0 & 167.9 & 144.5 & 156.2 & 129.4 & 128.3 & 128.8 \\
\hline $11: 49: \odot \odot$ & 709 & 141.6 & 142.4 & 142.0 & 167.9 & 147.4 & 157.6 & 128.3 & 127.2 & 127.8 \\
\hline $11: 5 \odot: \odot \odot$ & 710 & 141.6 & 141.4 & 1.5 & 166.5 & 146.1 & 156.3 & 128.3 & 127.2 & 127.8 \\
\hline $11: 51: \odot \odot$ & 711 & 141.6 & 141.4 & 1.5 & 166.5 & 146.1 & 156.3 & 128.3 & 127.2 & 127.8 \\
\hline $11: 52: 0 \odot$ & 712 & 141.6 & 141.4 & 141.5 & 166.5 & 147.7 & 157.1 & 128.3 & 127.2 & 127.8 \\
\hline $11: 53: \odot \odot$ & 713 & 141.6 & 141.4 & 141.5 & 166.5 & 147.6 & 157.0 & 128.3 & 127.2 & 127.8 \\
\hline $11: 54: \odot \odot$ & 714 & 140.5 & 141.4 & 140.9 & 166.5 & 147.6 & 157.0 & 128.3 & 127.2 & 127.8 \\
\hline $11: 55: \odot \odot$ & 715 & 140.5 & 141.4 & 140.9 & 166.5 & 146.3 & 156.4 & 128.3 & 127.2 & 127.8 \\
\hline $11: 56: \odot \odot$ & 716 & 140.5 & 141.4 & .9 & 166.5 & 147.4 & 156.9 & 128.3 & 127.2 & 127.8 \\
\hline $11: 57: \odot \odot$ & 717 & 140.5 & 141.4 & 140.9 & 166.1 & 146.1 & 156.1 & 128.3 & 127.2 & 127.8 \\
\hline $11: 58: 00$ & 718 & 140.5 & 141.4 & 140.9 & 166.1 & 146.5 & 156.3 & 127.2 & 127.2 & 127.2 \\
\hline $11: 59: \odot \odot$ & 719 & 140.5 & 140.3 & 140.4 & 166.1 & 144.5 & 155.3 & 127.2 & 127.2 & 127.2 \\
\hline $12: \odot \odot: \odot \odot$ & 720 & 140.5 & 140.3 & 140.4 & 166.1 & 145.8 & 155.9 & 127.2 & 126.1 & 126.7 \\
\hline $12: \odot 1: \odot \odot$ & 721 & 140.5 & 140.3 & 140.4 & 164.8 & 147.7 & 156.3 & 127.2 & 126.1 & 126.7 \\
\hline $12: \odot 2: \odot \odot$ & 722 & 140.5 & 140.3 & 140.4 & 164.8 & 144.3 & 154.6 & 127.2 & 126.1 & 126.7 \\
\hline $12: \odot 3: \odot \odot$ & 723 & 139.5 & 140.3 & 139.9 & 164.8 & 145.0 & 154.9 & 127.2 & 126.1 & 126.7 \\
\hline $12: \odot 4: \odot \odot$ & 724 & 139.5 & 14 & 139.9 & 164.8 & 145.6 & 155.2 & 127.2 & 126.1 & 126.7 \\
\hline $12: \odot 5: \odot \odot$ & 725 & 139.5 & 140.3 & 139.9 & 164.8 & 143.4 & 154.1 & 127.2 & 126.1 & 126.7 \\
\hline $12: \odot 6: \odot \odot$ & 726 & 139.5 & 139.3 & 139.4 & 164.8 & 142.2 & 153.5 & 127.2 & 126.1 & 126.7 \\
\hline $12: \odot 7: \odot \odot$ & 727 & 139.5 & 139.3 & 139.4 & 163.8 & 143.8 & 153.8 & 126.1 & 126.1 & 126.1 \\
\hline $12: \odot 8: \odot \odot$ & 728 & 139.5 & 139.3 & 139.4 & 163.8 & 145.4 & 154.6 & 126.1 & 126.1 & 126.1 \\
\hline $12: \odot 9: \odot \odot$ & 729 & 139.5 & 9.3 & 139.4 & 164.3 & 144.3 & 154.3 & 126.1 & 126.1 & 126.1 \\
\hline $12: 10: \odot \odot$ & 730 & 139.5 & 9.3 & 139.4 & 164.3 & 144.3 & 154.3 & 126.1 & 126.1 & 126.1 \\
\hline $12: 11: \odot \odot$ & 731 & 138.4 & 139.3 & 138.9 & 164.3 & 144.3 & 154.3 & 126.1 & 126.1 & 126.1 \\
\hline $12: 12: \odot \odot$ & 732 & 138.4 & 139.3 & 138.9 & 163.2 & 143.8 & 153.5 & 126.1 & 125.1 & 125.6 \\
\hline $12: 13: \odot \odot$ & 733 & 138.4 & 139.3 & 138.9 & 163.2 & 144.3 & 153.8 & 126.1 & 125.1 & 125.6 \\
\hline $12: 14: \odot \odot$ & 734 & 138.4 & 139.3 & 138.9 & 163.2 & 143.2 & 153.2 & 126.1 & 125.1 & 125.6 \\
\hline $12: 15: \odot \odot$ & 735 & 138.4 & 138.3 & 138.4 & 162.1 & 145.0 & 153.6 & 126.1 & 125.1 & 125.6 \\
\hline $12: 16: \odot \odot$ & 736 & 138.4 & 138.3 & 138.4 & 162.1 & 142.3 & 152.2 & 126.1 & 125.1 & 125.6 \\
\hline $12: 17: 00$ & 737 & 138.4 & 138.3 & 138.4 & 162.1 & 142.3 & 152.2 & 126.1 & 125.1 & 125.6 \\
\hline $12: 18: \odot \odot$ & 738 & 138.4 & 138.3 & 138.4 & 162.1 & 142.7 & 152.4 & 126.1 & 125.1 & 125.6 \\
\hline $12: 19: \odot \odot$ & 739 & 138.4 & 138.3 & 138.4 & 162.1 & 144.3 & 153.2 & 126.1 & 125.1 & 125.6 \\
\hline $12: 2 \odot: \odot \odot$ & 740 & 138.4 & 138.3 & 138.4 & 162.1 & 142.2 & 152.2 & 126.1 & 125.1 & 125.6 \\
\hline $12: 21: \odot \odot$ & 741 & 137.3 & 138.3 & 137.8 & 162.1 & 143.8 & 153.0 & 126.1 & 125.1 & 125.6 \\
\hline $12: 22: \odot \odot$ & 742 & 137.3 & 138.3 & 137.8 & 162.1 & 141.4 & 151.8 & 125.1 & 125.1 & 125.1 \\
\hline $12: 23: \odot \odot$ & 743 & 137.3 & 137.3 & 137.3 & 162.1 & 141.8 & 152.0 & 125.1 & 125.1 & 125.1 \\
\hline $12: 24: \odot \odot$ & 744 & 137.3 & 137.3 & 137.3 & 161.1 & 142.7 & 151.9 & 125.1 & 125.1 & 125.1 \\
\hline $12: 25: \odot \odot$ & 745 & 137.3 & 137.3 & 137.3 & 161.1 & 140.0 & 150.5 & 125.1 & 125.1 & 125.1 \\
\hline $12: 26: \odot \odot$ & 746 & 137.3 & 137.3 & 137.3 & 161.1 & 141.3 & 151.2 & 125.1 & 125.1 & 125.1 \\
\hline $12: 27: \odot \odot$ & 747 & 137.3 & 137.3 & 137.3 & 161.1 & 141.3 & 151.2 & 125.1 & 125.1 & 125.1 \\
\hline $12: 28: 0 \odot$ & 748 & 137.3 & 137.3 & 137.3 & 160.0 & 140.5 & 150.3 & 125.1 & 125.1 & 125.1 \\
\hline
\end{tabular}




\begin{tabular}{|c|c|c|c|c|c|c|c|c|c|c|}
\hline $12: 29: \odot \odot$ & 749 & 137.3 & 137.3 & 137.3 & 160.0 & 142.3 & 151.2 & 125.1 & 125.1 & 125.1 \\
\hline $12: 30: \odot \odot$ & 750 & 136.3 & 137.3 & 136.8 & 161.1 & 141.3 & 151.2 & 125.1 & 125.1 & 125.1 \\
\hline $12: 31: \odot \odot$ & 751 & 136.3 & 137.3 & 136.8 & 161.1 & 141.8 & 151.4 & 125.1 & 124.0 & 124.5 \\
\hline $12: 32: 0 \odot$ & 752 & 136.3 & 137.3 & 136.8 & 159.6 & 143.2 & 151.4 & 124.0 & 124.0 & 124.0 \\
\hline $12: 33: \odot \odot$ & 753 & 136.3 & 136.3 & 136.3 & 159.6 & 141.3 & 150.4 & 124.0 & 124.0 & 124.0 \\
\hline $12: 34: \odot \odot$ & 754 & 136.3 & 136.3 & 136.3 & 159.6 & 141.8 & 150.7 & 124.0 & 124.0 & 124.0 \\
\hline $2: 35: \odot \odot$ & 755 & 136.3 & 136.3 & 136.3 & 159.6 & 141.3 & 150.4 & 124.0 & 124.0 & 124.0 \\
\hline $12: 36: \odot \odot$ & 756 & 136.3 & 136.3 & 136.3 & 159.6 & 141.3 & 150.4 & 124.0 & 124.0 & 124.0 \\
\hline $12: 37: 0 \odot$ & 757 & 136.3 & 136.3 & 136.3 & 159.6 & 139.6 & 149.6 & 124.0 & 124.0 & 124.0 \\
\hline $12: 38: \odot \odot$ & 758 & 136.3 & 136.3 & 136.3 & 159.6 & 144.3 & 152.0 & 124.0 & 124.0 & 124.0 \\
\hline $12: 39: \odot \odot$ & 759 & 136.3 & 136.3 & 136.3 & 158.4 & 141.8 & 150.1 & 124.0 & 124.0 & 124.0 \\
\hline $12: 40: \odot \odot$ & 760 & 135.2 & 136.3 & 135.8 & 159.4 & 142.0 & 150.7 & 124.0 & 124.0 & 124.0 \\
\hline $12: 41: \odot \odot$ & 761 & 135.2 & 136.3 & 135.8 & 158.0 & 142.3 & 150.2 & 124.0 & 124.0 & 124.0 \\
\hline $12: 42: \odot \odot$ & 762 & 135.2 & 135.2 & 135.2 & 158.0 & 141.3 & 149.6 & 124.0 & 124.0 & 124.0 \\
\hline $12: 43: \odot \odot$ & 763 & 135.2 & 135.2 & 135.2 & 158.0 & 141.3 & 149.6 & 122.9 & 122.9 & 122.9 \\
\hline $12: 44: \odot \odot$ & 764 & 135.2 & 135.2 & 135.2 & 158.0 & 140.0 & 149.0 & 122.9 & 122.9 & 122.9 \\
\hline $12: 45: \odot \odot$ & 765 & 135.2 & 135.2 & 135.2 & 158.0 & 138.9 & 148.5 & 122.9 & 122.9 & 122.9 \\
\hline $12: 46: \odot \odot$ & 766 & 135.2 & 135.2 & 135.2 & 158.0 & 140.2 & 149.1 & 122.9 & 122.9 & 122.9 \\
\hline $12: 47: \odot \odot$ & 767 & 135.2 & 135.2 & 135.2 & 158.0 & 139.8 & 148.9 & 122.9 & 122.9 & 122.9 \\
\hline $12: 48: \odot \odot$ & 768 & 135.2 & 135.2 & 135.2 & 156.7 & 139.8 & 148.3 & 122.9 & 122.9 & 122.9 \\
\hline $12: 49: \odot \odot$ & 769 & 135.2 & 135.2 & 135.2 & 156.7 & 138.7 & 147.7 & 122.9 & 122.9 & 122.9 \\
\hline $12: 50: \odot \odot$ & 770 & 135.2 & 135.2 & 135.2 & 156.7 & 138.6 & 147.7 & 122.9 & 122.9 & 122.9 \\
\hline $12: 51: \odot \odot$ & 771 & 134.2 & 134.2 & 134.2 & 157.8 & 141.6 & 149.7 & 122.9 & 122.9 & 122.9 \\
\hline $12: 52: \odot \odot$ & 772 & 134.2 & 134.2 & 134.2 & 156.7 & 138.2 & 147.5 & 122.9 & 122.9 & 122.9 \\
\hline $12: 53: \odot \odot$ & 773 & 134.2 & 134.2 & 134.2 & 156.7 & 138.9 & 147.8 & 122.9 & 122.9 & 122.9 \\
\hline $12: 54: \odot \odot$ & 774 & 134.2 & 134.2 & 134.2 & 156.7 & 141.3 & 149.0 & 122.9 & 122.9 & 122.9 \\
\hline $12: 55: \odot \odot$ & 775 & 134.2 & 134.2 & 134.2 & 156.7 & 139.1 & 147.9 & 122.9 & 122.9 & 122.9 \\
\hline $12: 56: 0 \odot$ & 776 & 134.2 & 134.2 & 134.2 & 155.7 & 137.7 & 146.7 & 122.9 & 121.8 & 122.4 \\
\hline $12: 57: \odot \odot$ & 777 & 134.2 & 134.2 & 134.2 & 156.7 & 137.7 & 147.2 & 121.8 & 121.8 & 121.8 \\
\hline $12: 58: \odot \odot$ & 778 & 134.2 & 134.2 & 134.2 & 156.7 & 139.6 & 148.2 & 121.8 & 121.8 & 121.8 \\
\hline $12: 59: \odot \odot$ & 779 & 133.2 & 134.2 & 133.7 & 156.7 & 138.4 & 147.6 & 121.8 & 121.8 & 121.8 \\
\hline $13: \odot \odot: \odot \odot$ & 780 & 133.2 & 134.2 & 133.7 & 155 & 139.8 & 147.7 & 121.8 & 121.8 & 121.8 \\
\hline $13: \odot 1: \odot \odot$ & 781 & 133.2 & 133.2 & 133.2 & 155.7 & 138.4 & 147.0 & 121.8 & 121.8 & 121.8 \\
\hline $13: 02: 00$ & 782 & 133.2 & 133.2 & 133.2 & 155.7 & 137.1 & 146.4 & 121.8 & 121.8 & 121.8 \\
\hline $13: \odot 3: \odot \odot$ & 783 & 133.2 & 133.2 & 133.2 & 154.6 & 139.5 & 147.0 & 121.8 & 121.8 & 121.8 \\
\hline $13: \odot 4: \odot \odot$ & 784 & 133.2 & 133.2 & 133.2 & 154.6 & 138.2 & 146.4 & 121.8 & 121.8 & 121.8 \\
\hline $13: \odot 5: \odot \odot$ & 785 & 133.2 & 133.2 & 133.2 & 155.3 & 138.7 & 147.0 & 121.8 & 121.8 & 121.8 \\
\hline $13: \odot 6: \odot \odot$ & 786 & 133.2 & 133.2 & 133.2 & 155.3 & 138.9 & 147.1 & 121.8 & 120.7 & 121.3 \\
\hline $13: \odot 7: \odot \odot$ & 787 & 133.2 & 133.2 & 133.2 & 155.3 & 136.8 & 146.0 & 120.7 & 120.7 & 120.7 \\
\hline $13: 08: 00$ & 788 & 133.2 & 133.2 & 133.2 & 154.2 & 139.1 & 146.7 & 120.7 & .7 & 120.7 \\
\hline $13: \odot 9: \odot \odot$ & 789 & 133.2 & 133.2 & 133.2 & 154.2 & 139.5 & 146.8 & 120.7 & 120.7 & 120.7 \\
\hline $13: 10: \odot \odot$ & 790 & 132.1 & 132.2 & 132.1 & 154.2 & 135.3 & 144.8 & 120.7 & 120.7 & 120.7 \\
\hline $13: 11: \odot \odot$ & 791 & 132.1 & 132.2 & 132.1 & 153.1 & 136.8 & 145.0 & 120.7 & 120.7 & 120.7 \\
\hline $13: 12: \odot \odot$ & 792 & 132.1 & 132.2 & 132.1 & 154.2 & 139.5 & 146.8 & 120.7 & 120.7 & 120.7 \\
\hline $13: 13: \odot \odot$ & 793 & 132.1 & 132.2 & 132.1 & 153.1 & 137.8 & 145.5 & 120.7 & 120 & 120.7 \\
\hline $13: 14: \odot \odot$ & 794 & 132.1 & 132.2 & 132.1 & 154.2 & 136.9 & 145.6 & 120.7 & 120.7 & 120.7 \\
\hline $13: 15: 00$ & 795 & 132.1 & 132.2 & 132.1 & 153.1 & 138.2 & 145.7 & 120.7 & 120.7 & 120.7 \\
\hline $13: 16: \odot \odot$ & 796 & 132.1 & 132.2 & 132.1 & 153.1 & 136.9 & 145.0 & 120.7 & 120.7 & 120.7 \\
\hline $13: 17: \odot \odot$ & 797 & 132.1 & 132.2 & 132.1 & 153.1 & 136.9 & 145.0 & 120.7 & 120.7 & 120.7 \\
\hline $13: 18: \odot \odot$ & 798 & 132.1 & 132.2 & 132.1 & 153.1 & 136.9 & 145.0 & 120.7 & 120.7 & 120.7 \\
\hline $13: 19: \odot \odot$ & 799 & 132.1 & 132.2 & 132.1 & 153.1 & 136.9 & 145.0 & 120.7 & 120.7 & 120.7 \\
\hline $13: 20: \odot \odot$ & 800 & 131.0 & 131.1 & 131.1 & 152.1 & 136.9 & 144.5 & 120.7 & 120.7 & 120.7 \\
\hline $13: 21: 00$ & 801 & 131.0 & 131.1 & 131.1 & 152.1 & 135.7 & 143.9 & 119.7 & 120.7 & 120.2 \\
\hline $13: 22: \odot \odot$ & 802 & 131.0 & 131.1 & 131.1 & 152.1 & 135.5 & 143.8 & 119.7 & 120.7 & 120.2 \\
\hline $13: 23: \odot \odot$ & 803 & 131.0 & 131.1 & 131.1 & 152.1 & 135.7 & 143.9 & 119.7 & 120.7 & 120.2 \\
\hline $13: 24: \odot \odot$ & 804 & 131.0 & 131.1 & 131.1 & 152.1 & 136.8 & 144.4 & 119.7 & 120.7 & 120.2 \\
\hline $13: 25: \odot \odot$ & 805 & 131.0 & 131.1 & 131.1 & 152.1 & 135.5 & 143.8 & 119.7 & 119.7 & 119.7 \\
\hline $13: 26: \odot \odot$ & 806 & 131.0 & 131.1 & 131.1 & 152.1 & 135.1 & 143.6 & 119.7 & 119.7 & 119.7 \\
\hline $13: 27: \odot \odot$ & 807 & 131.0 & 131.1 & 131.1 & 152.1 & 138.2 & 145.1 & 119.7 & 119.7 & 119.7 \\
\hline $13: 28: \odot \odot$ & 808 & 131.0 & 131.1 & 131.1 & 152.1 & 135.7 & 143.9 & 119.7 & 119.7 & 119.7 \\
\hline $13: 29: \odot \odot$ & 809 & 131.0 & 131.1 & 131.1 & 151.5 & 135.7 & 143.6 & 119.7 & 119.7 & 119.7 \\
\hline $13: 3 \odot: \odot \odot$ & 810 & 131.0 & 130.1 & 130.6 & 151.5 & 136.9 & 144.2 & 119.7 & 119.7 & 119.7 \\
\hline $13: 31: \odot \odot$ & 811 & 130.0 & 130.1 & 130.1 & 151.5 & 134.4 & 143.0 & 119.7 & 119.7 & 119.7 \\
\hline $13: 32: 00$ & 812 & 130.0 & 130.1 & 130.1 & 151.5 & 134.2 & 142.9 & 119.7 & 119.7 & 119.7 \\
\hline
\end{tabular}




\begin{tabular}{|c|c|c|c|c|c|c|c|c|c|c|}
\hline $13: 33: \odot \odot$ & 813 & 130.0 & 130.1 & 130.1 & 151.5 & 134.8 & 143.2 & 119.7 & 119.7 & 119.7 \\
\hline $13: 34: \odot \odot$ & 814 & 130.0 & 130.1 & 130.1 & 150.1 & 138.0 & 144.1 & 119.7 & 119.7 & 119.7 \\
\hline $13: 35: \odot \odot$ & 815 & 130.0 & 130.1 & 130.1 & 150.1 & 136.6 & 143.3 & 119.7 & 119.7 & 119.7 \\
\hline $13: 36: \odot \odot$ & 816 & 130.0 & 130.1 & 130.1 & 150.1 & 134.8 & 142.4 & 119.7 & 119.7 & 119.7 \\
\hline $13: 37: \odot \odot$ & 817 & 130.0 & 130.1 & 130.1 & 150.8 & 134.8 & 142.8 & 118.6 & 119.7 & 119.1 \\
\hline $13: 38: \odot \odot$ & 818 & 130.0 & 130.1 & 130.1 & 150.8 & 134.8 & 142.8 & 118.6 & 118.6 & 118.6 \\
\hline $13: 39: \odot \odot$ & 819 & 130.0 & 130.1 & 130.1 & 149.7 & 133.7 & 141.7 & 118.6 & 118.6 & 118.6 \\
\hline $13: 4 \odot: \odot \odot$ & 820 & 130.0 & 130.1 & 130.1 & 149.7 & 133.3 & 141.5 & 118.6 & 118.6 & 118.6 \\
\hline $13: 41: \odot \odot$ & 821 & 130.0 & 130.1 & 130.1 & 149.7 & 135.0 & 142.3 & 118.6 & 118.6 & 118.6 \\
\hline $3: 42: 00$ & 22 & 128.9 & 129.1 & 129.0 & 149.7 & 135.0 & 142.3 & 118.6 & 118.6 & 118.6 \\
\hline $3: 43: \odot \odot$ & 823 & 128.9 & 129.1 & 129.0 & 149.7 & 134.6 & 142.2 & 118.6 & 118.6 & 118.6 \\
\hline $13: 48: \odot \odot$ & 828 & 128.9 & 129.1 & 129.0 & 148.5 & 131.0 & 139.7 & 117.5 & 117.5 & 117.5 \\
\hline $13: 53: \odot \odot$ & 833 & 128.9 & 129.1 & 129.0 & 148.5 & 135.0 & 141.7 & 117.5 & 117.5 & 117.5 \\
\hline $13: 58: \odot \odot$ & 838 & 127.9 & 128.0 & 127.9 & 148.5 & 133.7 & 141.1 & 117.5 & 116.4 & 117.0 \\
\hline $14: \odot 3: \odot \odot$ & 843 & 127.9 & 128.0 & 127.9 & 147.0 & 133.3 & 140.2 & 116.4 & 116.4 & 116.4 \\
\hline $4: \odot 8: \odot \odot$ & 848 & 126.8 & 127.0 & 126.9 & 147.0 & 134.1 & 140.5 & 116.4 & 115.3 & 115.9 \\
\hline $14: 13: \odot \odot$ & 853 & 126.8 & 127.0 & 126.9 & 146.1 & 133.3 & 139.7 & 115.3 & 115.3 & 115.3 \\
\hline $14: 18: 00$ & 858 & 125.7 & 126.0 & 125.9 & 146.1 & 133.7 & 139.9 & 115.3 & 115.3 & 115.3 \\
\hline $14: 23: \odot \odot$ & 863 & 125.7 & 126.0 & 125.9 & 144.0 & 129.7 & 136.9 & 115.3 & 115.3 & 115.3 \\
\hline $14: 28: \odot \odot$ & 868 & 125.7 & 124.9 & 125.3 & 142.9 & 132.8 & 137.8 & 115.3 & 115.3 & 115.3 \\
\hline $14: 33: 0 \odot$ & 873 & 124.7 & 124.9 & 124.8 & 142.9 & 131.5 & 137.2 & 114.3 & 114.3 & 114.3 \\
\hline $14: 38: \odot \odot$ & 878 & 124.7 & 124.9 & 124.8 & 141.8 & 128.5 & 135.1 & 114.3 & 114.3 & 114.3 \\
\hline $14: 43: \odot \odot$ & 883 & 123.6 & 123.9 & 123.8 & 141.8 & 129.7 & 135.8 & 114.3 & 114 & 114.3 \\
\hline $14: 48: \odot \odot$ & 888 & 123.6 & 123.9 & 123.8 & 141.8 & 127.6 & 134.7 & 113.2 & 114.3 & 113.7 \\
\hline $14: 53: \odot \odot$ & 893 & 123.6 & 122.9 & 123.3 & 140.5 & 129.2 & 134.9 & 113.2 & 113.2 & 113.2 \\
\hline $14: 58: \odot \odot$ & 898 & 122.5 & 122.9 & 122.7 & 139.5 & 127.8 & 133.6 & 113.2 & 113.2 & 113.2 \\
\hline $15: \odot 3: \odot \odot$ & 903 & 122.5 & 121.9 & 122.2 & 139.5 & 126.7 & 133.1 & 112.1 & 113.2 & 112.6 \\
\hline $15: \odot 8: \odot \odot$ & 908 & 121.5 & 121.9 & 121.7 & 138.2 & 127.0 & 132.6 & 112.1 & 113.2 & 112.6 \\
\hline $15: 13: \odot \odot$ & 913 & 121.5 & 121.9 & 121.7 & 138.2 & 126.3 & 132.3 & 111.0 & 112.1 & 111.6 \\
\hline $15: 18: \odot \odot$ & 918 & 121.5 & 120.9 & 121.2 & 136.9 & 127.2 & 132.1 & 111.0 & 112.1 & 111.6 \\
\hline $15: 23: \odot \odot$ & 923 & 120.5 & 120.9 & 120.7 & 136.8 & 126.3 & 131.5 & 111.0 & 112.1 & 111.6 \\
\hline $15: 28: \odot \odot$ & 928 & 120.5 & 120.9 & 120.7 & 135.7 & 126.3 & 131.0 & 111.0 & 112.1 & 111.6 \\
\hline $15: 33: \odot \odot$ & 933 & 120.5 & 119.9 & 120.2 & 135.7 & 124.7 & 130.2 & 111.0 & 111.0 & 111.0 \\
\hline $15: 38: \odot \odot$ & 938 & 119.4 & 119.9 & 119.7 & 135.7 & 124.7 & 130.2 & 109.9 & 111.0 & 110.5 \\
\hline $15: 43: \odot \odot$ & 943 & 119.4 & 118.9 & 119.2 & 134.6 & 124.7 & 129.7 & 109.9 & 111.0 & 110.5 \\
\hline $15: 48: \odot \odot$ & 948 & 119.4 & 118.9 & 119.2 & 134.6 & 123.8 & 129.2 & 108.9 & 109 & 109.4 \\
\hline $15: 53: \odot \odot$ & 953 & 118.4 & 118.9 & 118.6 & 133.5 & 123.8 & 128.7 & 108.9 & 109.9 & 109.4 \\
\hline $15: 58: \odot \odot$ & 958 & 118.4 & 117.9 & 118.1 & 133.5 & 123.3 & 128.4 & 108.9 & 109.9 & 109.4 \\
\hline $16: 03: \odot \odot$ & 963 & 117.4 & 117.9 & 117.6 & 132.3 & 123.1 & 127.7 & 108.9 & 108.9 & 108.9 \\
\hline $16: \odot 8: \odot \odot$ & 968 & 117.4 & 117.9 & 117.6 & 133.3 & 123.1 & 128.2 & 107.8 & 108.9 & 108.3 \\
\hline $16: 13: \odot \odot$ & 973 & 117.4 & 116.9 & 117.1 & 132.3 & 121.6 & 127.0 & 107.8 & 107.8 & 107.8 \\
\hline $16: 18: \odot \odot$ & 978 & 116.3 & 116.9 & 116.6 & 131.0 & 121.5 & 126.2 & 107.8 & 107.8 & 107.8 \\
\hline $16: 23: \odot \odot$ & 983 & 116.3 & 115.9 & 116.1 & 131.0 & 122.4 & 126.7 & 106.7 & 107.8 & 107.2 \\
\hline $16: 28: \odot \odot$ & 988 & 116.3 & 115.9 & 116.1 & 129.9 & 120.9 & 125.4 & 106.7 & 106.7 & 106.7 \\
\hline $16: 33: \odot \odot$ & 993 & 115.3 & 115.9 & 115.6 & 129.9 & 121.3 & 125.6 & 105.6 & 106.7 & 106.2 \\
\hline $16: 38: \odot \odot$ & 998 & 115.3 & 114.8 & 115.1 & 128.8 & 120.0 & 124.4 & 105.6 & 106.7 & 106.2 \\
\hline $16: 43: 00$ & 1003 & 115.3 & 114.8 & 115.1 & 127.8 & 120.7 & 124.3 & 105.6 & 106.7 & 106.2 \\
\hline $16: 48: \odot \odot$ & 1008 & 114.2 & 114.8 & 114.5 & 127.9 & 119.7 & 123.8 & 105.6 & 105.6 & 105.6 \\
\hline $16: 53: \odot \odot$ & 1013 & 114.2 & 113.8 & 114.0 & 127.6 & 118.0 & 122.8 & 105.6 & 105.6 & 105.6 \\
\hline $16: 58: 0 \odot$ & 1018 & 114.2 & 113.8 & 114.0 & 127.6 & 118.8 & 123.2 & 104.5 & 105.6 & 105.1 \\
\hline $17: \odot 3: \odot \odot$ & 1023 & 114.2 & 113.8 & 114.0 & 127.4 & 118.9 & 123.2 & 104.5 & 105.6 & 105.1 \\
\hline $17: \odot 8: \odot \odot$ & 1028 & 113.0 & 112.7 & 112.9 & 126.3 & 118.8 & 122.5 & 104.5 & 105.6 & 105.1 \\
\hline $17: 13: \odot \odot$ & 1033 & 113.0 & 112.7 & 112.9 & 125.2 & 118.0 & 121.6 & 104.5 & 105.6 & 105.1 \\
\hline $17: 18: \odot \odot$ & 1038 & 113.0 & 112.7 & 112.9 & 125.2 & 117.1 & 121.2 & 103.5 & 105.6 & 104.5 \\
\hline $17: 23: \odot \odot$ & 1043 & 112.0 & 111.7 & 111.8 & 124.2 & 118.2 & 121.2 & 103.5 & 105.6 & 104.5 \\
\hline $17: 28: \odot \odot$ & 1048 & 112.0 & 111.7 & 111.8 & 124.2 & 118.8 & 121.5 & 103.5 & 105.6 & 104.5 \\
\hline $17: 33: \odot \odot$ & 1053 & 112.0 & 111.7 & 111.8 & 124.2 & 117.5 & 120.8 & 103.5 & 104.5 & 104.0 \\
\hline $17: 38: \odot \odot$ & 1058 & 112.0 & 111.7 & 111.8 & 123.1 & 117.5 & 120.3 & 103.5 & 104.5 & 104.0 \\
\hline $17: 43: \odot \odot$ & 1063 & 110.9 & 110.7 & 110.8 & 123.1 & 118.0 & 120.6 & 103.5 & 104.5 & 104.0 \\
\hline $17: 48: \odot \odot$ & 1068 & 110.9 & 110.7 & 110.8 & 122.0 & 115.7 & 118.9 & 103.5 & 104.5 & 104.0 \\
\hline $17: 53: \odot \odot$ & 1073 & 110.9 & 110.7 & 110.8 & $122 . \odot$ & 116.4 & 119.2 & 102.4 & 103.5 & 102.9 \\
\hline $17: 58: \odot \odot$ & 1078 & 109.9 & 109.7 & 109.8 & 122.0 & 116.4 & 119.2 & 102.4 & 103.5 & 102.9 \\
\hline $18: \odot 3: \odot \odot$ & 1083 & 109.9 & 109.7 & 109.8 & 121.8 & 115.3 & 118.6 & 102.4 & 103.5 & 102.9 \\
\hline 18: $08: \odot \odot$ & 1088 & 109.9 & 109.7 & 109.8 & 120.7 & 116.1 & 118.4 & 102.4 & 103.5 & 102.9 \\
\hline
\end{tabular}




\begin{tabular}{|c|c|c|c|c|c|c|c|c|c|c|}
\hline $18: 13: \odot \odot$ & 1093 & 109.9 & 109.7 & 109.8 & 120.7 & 117.0 & 118.9 & 102.4 & 103.5 & 102.9 \\
\hline $18: 18: \odot \odot$ & 1098 & 108.9 & 109.7 & 109.3 & 120.7 & 114.4 & 117.6 & 102.4 & 103.5 & 102.9 \\
\hline $18: 23: \odot \odot$ & 1103 & 108.9 & 108.7 & 108.8 & 119.7 & 114.4 & 117.1 & 101.3 & 103.5 & $10<2$ \\
\hline $18: 28: \odot \odot$ & 1108 & 108.9 & 108.7 & 108.8 & 119.7 & 114.1 & 116.9 & 101.3 & 103.5 & 102.2 \\
\hline $18: 33: \odot \odot$ & 1113 & 108.9 & 108.7 & 108.8 & 119.7 & 113.2 & 116.4 & 101.3 & 102.4 & 101. \\
\hline $18: 38: \odot \odot$ & 118 & 107.7 & 107.7 & 107.7 & 118.4 & 113.7 & 116.1 & 101.3 & 102.4 & 101.8 \\
\hline $18: 43: \odot \odot$ & 1123 & 107.7 & 107.7 & 107.7 & 118.2 & 114.1 & 116.2 & 100.2 & 101.3 & ๑๑. \\
\hline $18: 48: \odot \odot$ & 128 & 107.7 & 107.7 & 107.7 & 118.2 & 112.6 & 115.4 & 100.2 & 101.3 & ૫. \\
\hline $18: 53: \odot \odot$ & 133 & -07.7 & 107.7 & 107.7 & 117.1 & 112.8 & 115.0 & 100.2 & 101.3 & $\odot \odot$. \\
\hline $18: 58: \odot \odot$ & 138 & 107.7 & 107.7 & 107.7 & 117.1 & 111.6 & 114.4 & 100.2 & 101.3 & 00. \\
\hline $19: \odot 3: \odot \odot$ & 143 & 06.7 & 106.6 & 106.6 & 117.1 & 112.6 & 114.9 & 100.2 & 101.3 & 00. \\
\hline 19:๑8:๑९ & 1148 & 106.7 & 106.6 & 106.6 & 115.9 & 112.6 & 114.3 & 100.2 & 101.3 & 100 \\
\hline $19: 13: \odot \odot$ & 1153 & 106.7 & 106.6 & 106.6 & 115.9 & 112.8 & 114.4 & 100.2 & 101.3 & 100. \\
\hline $19: 18: \odot \odot$ & 1158 & 106.7 & 106.6 & 106.6 & 115.9 & 111.7 & 113.8 & 99.1 & 101.3 & 100 \\
\hline $19: 23: \odot \odot$ & 163 & 106.7 & 105.6 & 106.1 & 114.8 & 111.7 & 113.3 & 99.1 & 101.3 & \\
\hline $19: 28: \odot \odot$ & 168 & 105.6 & 105.6 & 105.6 & 114.8 & 110.7 & 112.7 & 99.1 & 100.2 & 99.7 \\
\hline $19: 33: \odot \odot$ & 173 & 105.6 & 105.6 & 105.6 & 114.8 & 111.0 & 112.9 & 99.1 & 100.2 & 99.7 \\
\hline $19: 3$ & 1178 & 105.6 & 105.6 & 105.6 & 114.8 & 111.0 & 112.9 & 99.1 & 100.2 & 99.7 \\
\hline $19: 43: \odot \odot$ & 1183 & 105.6 & 104.6 & 105.1 & 113.7 & 111.0 & 112.4 & 99.1 & 100.2 & 99.7 \\
\hline $19: 48: \odot \odot$ & 1188 & 104.6 & 104.6 & 104.6 & 113.7 & 109.9 & 111.8 & & & 99.1 \\
\hline$: \odot \odot$ & 193 & 104.6 & 104.6 & 104.6 & 113.7 & 109.9 & 111.8 & & & 8.6 \\
\hline :०९ & 198 & .04 .6 & 104.6 & 104.6 & 112.6 & 109.9 & & & & 98.6 \\
\hline $20: \odot$ & 203 & 104.6 & 104.6 & 104.6 & 112.6 & 109.4 & 111.0 & 1 & .1 & 98.6 \\
\hline $20: \Theta$ & 208 & 104.6 & 103.6 & 104.1 & 112.6 & 107.4 & & & & 98.6 \\
\hline $20: 13: \odot \odot$ & 1213 & 103.5 & 103.6 & 103.5 & 112.6 & 109.8 & & & & 98.6 \\
\hline $20: 18: \odot \odot$ & 1218 & 103.5 & 103.6 & 103.5 & 111.6 & 108.7 & 110.1 & 98.1 & 9.1 & 98.6 \\
\hline $20: 2$ & 1223 & 103.5 & 103.6 & 103.5 & 111.6 & 108.7 & 110.1 & 97.0 & 8.1 & 97.5 \\
\hline $20: 2$ & 1228 & 103.5 & 103.6 & 103.5 & 111.6 & 107.6 & 109.6 & & .1 & 97.5 \\
\hline $20: 3$ & 1233 & 103.5 & 102.6 & 103.0 & 110.3 & 108.1 & 109.2 & 97.0 & 98.1 & 97.5 \\
\hline $20: 3$ & 1238 & 103.5 & 102.6 & 103.0 & 110.3 & 108.1 & 109.2 & & 98.1 & 97.5 \\
\hline $20: 4$ & 243 & 102.5 & 102.6 & 102.5 & 110.3 & 107.1 & 108.7 & 97.0 & & 97.5 \\
\hline $20: 4$ & 248 & & 102.6 & & 110.3 & 107.1 & & & & 97.0 \\
\hline $20: 5$ & 253 & 02.5 & 102.6 & 102.5 & 109.2 & 107.2 & & & & 96.4 \\
\hline $20: 5$ & 258 & 102.5 & 101.6 & 102.0 & 109.2 & 107.2 & & & & 96.4 \\
\hline $21: 0$ & 263 & 102.5 & 101.6 & 102.0 & 109.2 & 107.2 & 8.2 & & & 96.4 \\
\hline & & 101.4 & 101.6 & & 109.2 & & & & & 96.4 \\
\hline $21: 1$ & 273 & 101.4 & 101.6 & 101.5 & 108.1 & 106.2 & 107.2 & 5. & 7.0 & 96.4 \\
\hline $21:-$ & 78 & 101.4 & 101.6 & & & & & & & 6.4 \\
\hline $21: 2$ & 283 & 101.4 & 100.6 & 101.0 & 108.1 & 105.8 & 7.0 & & & 95.4 \\
\hline $21: 2$ & 1288 & 101.4 & 100.6 & 101.0 & 107.1 & 104.7 & 105.9 & 94.8 & 95.9 & 95.4 \\
\hline & & & & & 107.1 & & & & & 95.4 \\
\hline $21: 3$ & 298 & 100.4 & 100.6 & 100.5 & 107.1 & 105.3 & 106.2 & 94.8 & 95.9 & 95.4 \\
\hline & & & & & & & & & & 95.4 \\
\hline $21: 4$ & 08 & .4 & 100.6 & 100.5 & 106.0 & 104.2 & 105.1 & 94. & & 95.4 \\
\hline & & & & & & & & & & 94.8 \\
\hline & & & & & & & & & & 94.8 \\
\hline $22: 6$ & 323 & & 99.6 & 99 & 106.0 & 104.2 & & & & 94.3 \\
\hline & & & & & & & & & & \\
\hline $22:$ & 33 & 3 & 99.6 & & & & & & & 94.3 \\
\hline & & & & & & & & & & \\
\hline $22: 2$ & 343 & & 98.5 & 98 & 104.9 & 103.3 & 104.1 & & & 94.3 \\
\hline & & & & & & & & & & \\
\hline $22: 3$ & 3 & & 98.5 & 98 & 104.9 & 103.1 & 104.0 & 93. & 94 & 94.3 \\
\hline $22: 3$ & & & & & & & & & & \\
\hline & & & & & & & & & & 94.3 \\
\hline $22: 4$ & & & & & & & & & & 93.2 \\
\hline & & & & & & & & & & 93.2 \\
\hline $22: 5$ & & & & & & & & & & 93.2 \\
\hline & & & & & & & & & & \\
\hline $23: \odot \varepsilon$ & & & & & 103.6 & 102.0 & 102.8 & & & 93.2 \\
\hline & & & & & & & & & & \\
\hline $23: 18: \odot \odot$ & & & & & & & 102.3 & & & 93.2 \\
\hline & & & & & & & & & & \\
\hline & & & & & & & & 2.7 & 93.7 & \\
\hline
\end{tabular}




\begin{tabular}{|c|c|c|c|c|c|c|c|c|c|c|}
\hline $23: 33: \odot \odot$ & 1413 & 97.2 & 97.5 & 97.4 & 102.6 & 102.0 & 102.3 & 92.7 & 93.7 & 93.2 \\
\hline $23: 38: \odot \odot$ & 1418 & 97.2 & 96.4 & 96.8 & 101.5 & 102.0 & 101.8 & 92.7 & 93.7 & 93.2 \\
\hline $23: 43: \odot \odot$ & 1423 & 97.2 & 96.4 & 96.8 & 101.5 & 102.0 & 101.8 & 92.7 & 93.7 & 93.2 \\
\hline $23: 48: \odot \odot$ & 1428 & 97.2 & 96.4 & 96.8 & 101.5 & 102.0 & 101.8 & 92.7 & 93.7 & 93.2 \\
\hline $23: 53: \odot \odot$ & 1433 & 97.2 & 96.4 & 96.8 & 101.5 & 101.7 & 101.6 & 92.7 & 93.7 & 93.2 \\
\hline $23: 58: \odot \odot$ & 1438 & 97.2 & 96.4 & 96.8 & 101.5 & 101.7 & 101.6 & 92.7 & 93.7 & 93.2 \\
\hline$\odot: \odot 3: \odot \odot$ & 1443 & 96.2 & 96.4 & 96.3 & 101.5 & 100.6 & 101.0 & 92.7 & 93.7 & 93.2 \\
\hline$\odot: \odot ৪: \odot \odot$ & 1448 & 96.2 & 96.4 & 96.3 & 101.5 & 100.6 & 101.0 & 92.7 & 93.7 & 93.2 \\
\hline$\odot: 13: \odot \odot$ & 1453 & 96.2 & 96.4 & 96.3 & 101.5 & 101.8 & 101.7 & 92.7 & 93.7 & 93.2 \\
\hline$\odot: 18: \odot \odot$ & 1458 & 96.2 & 96.4 & 96.3 & 100.4 & 99.7 & 100.0 & 92.7 & 93.7 & 93.2 \\
\hline$\odot: 23: \odot \odot$ & 1463 & 96.2 & 96.4 & 96.3 & 100.4 & 101.5 & 100.9 & 92.7 & 93.7 & 93.2 \\
\hline$\odot: 28: \odot \odot$ & 1468 & 96.2 & 95.4 & 95.8 & 100.4 & 100.0 & 100.2 & 92.7 & 93.7 & 93.2 \\
\hline$\odot: 33: \odot \odot$ & 1473 & 96.2 & 95.4 & 95.8 & 100.4 & 100.0 & 100.2 & 92.7 & 92.7 & 92.7 \\
\hline$\odot: 38: \odot \odot$ & 1478 & 96.2 & 95.4 & 95.8 & 100.4 & 100.0 & 100.2 & 92.7 & 92.7 & 92.7 \\
\hline$\odot: 43: \odot \odot$ & 1483 & 96.2 & 95.4 & 95.8 & 100.4 & 100.0 & 100.2 & 92.7 & 92.7 & 92.7 \\
\hline$\odot: 48: \odot \odot$ & 1488 & 96.2 & 95.4 & 95.8 & 99.3 & 98.8 & 99.1 & 91.6 & 92.7 & 92.1 \\
\hline$\odot: 53: \odot \odot$ & 1493 & 95.1 & 95.4 & 95.3 & 99.3 & 99.9 & 99.6 & 91.6 & 92.7 & 92.1 \\
\hline$\odot: 58: \odot \odot$ & 1498 & 95.1 & 95.4 & 95.3 & 99.3 & 99.9 & 99.6 & 91.6 & 92.7 & 92.1 \\
\hline 1:๑3:๑९ & 1503 & 95.1 & 95.4 & 95.3 & 99.3 & 99.7 & 99.5 & 91.6 & 92.7 & 92.1 \\
\hline 1: $\odot: \odot \odot$ & 1508 & 95.1 & 95.4 & 95.3 & 99.3 & 99.7 & 99.5 & 91.6 & 92.7 & 92.1 \\
\hline $1: 13: \odot \odot$ & 1513 & 95.1 & 94.4 & 94.8 & 99.3 & 99.7 & 99.5 & 91.6 & 91.6 & 91.6 \\
\hline $1: 18: \odot \odot$ & 1518 & 95.1 & 94.4 & 94.8 & 98.2 & 98.6 & 98.4 & 91.6 & 91.6 & 91.6 \\
\hline $1: 23: \odot \odot$ & 1523 & 95.1 & 94.4 & 94.8 & 98.2 & 98.6 & 98.4 & 90.5 & 91.6 & 91.0 \\
\hline $1: 28: \odot \odot$ & 1528 & 95.1 & 94.4 & 94.8 & 98.2 & 98.4 & 98.3 & 90.5 & 91.6 & 91.0 \\
\hline $1: 33: \odot \odot$ & 1533 & 95.1 & 94.4 & 94.8 & 98.2 & 98.2 & 98.2 & 90.5 & 91.6 & 91.0 \\
\hline $1: 38: \odot \odot$ & 1538 & 95.1 & 94.4 & 94.8 & 98.2 & 98.2 & 98.2 & 90.5 & 91.6 & 91.0 \\
\hline $1: 43$ & 1543 & 94.1 & 94.4 & 94.3 & 98.2 & 98.2 & 98.2 & 90.5 & 91.6 & 91.0 \\
\hline $1: 48: \odot \odot$ & 1548 & 94.1 & 94.4 & 94.3 & 98.2 & 98.2 & 98.2 & 90.5 & 91.6 & 91.0 \\
\hline $1: 53: 0 \odot$ & 1553 & 94.1 & 94.4 & 94.3 & 97.2 & 98.2 & 97.7 & 90.5 & 91.6 & 91.0 \\
\hline 1:58:๑๑ & 1558 & 94.1 & 93.4 & 93.7 & 97.2 & 98.2 & 97.7 & 90.5 & 91.6 & 91.0 \\
\hline $2: \odot 3: \odot \odot$ & 563 & 94.1 & 93.4 & 93.7 & 97.2 & 98.2 & 97.7 & 90.5 & 91.6 & 91.0 \\
\hline 2:०৪:๑९ & 1568 & 94.1 & 93.4 & 93.7 & 97.2 & 98.2 & & 90.5 & 91.6 & 91.0 \\
\hline $2: 13: \odot \odot$ & 1573 & 94.1 & 93.4 & 93.7 & 97.2 & 98.2 & 97 & 90.5 & 91.6 & 91.0 \\
\hline $2: 18: \odot \odot$ & 1578 & 94.1 & 93.4 & 93.7 & 97.2 & 97.2 & 97.2 & 90.5 & 90.5 & 90.5 \\
\hline $2: 23$ & 1583 & 94.1 & 93.4 & 93.7 & 97.2 & 97.2 & 97.2 & 89.4 & 90.5 & $9 \odot . \odot$ \\
\hline $2: 28$ & 1588 & 94.1 & 93.4 & 93.7 & 96.1 & 97.2 & 96.6 & 89.4 & 90.5 & $90 . \odot$ \\
\hline $2: 3$ & 1593 & 94.1 & 93.4 & 93.7 & 96.1 & 97.2 & 96.6 & 89.4 & 90.5 & $90 . \odot$ \\
\hline $2: 38: \odot \odot$ & 1598 & 93.0 & 93.4 & 93.2 & 96.1 & 97.2 & 96.6 & 89.4 & 90.5 & 90.0 \\
\hline & 603 & 93.0 & & & & & & 89.4 & 90.5 & 90.0 \\
\hline $2: 4 \varepsilon$ & 608 & 93.0 & 93.4 & 93.2 & 96.1 & 95.9 & 96.0 & 89.4 & 90.5 & $90 . \odot$ \\
\hline & 613 & & 92. & & & & & 89.4 & 90.5 & $90 . \odot$ \\
\hline $2: 5 \varepsilon$ & 618 & 93.0 & 92.3 & 92.7 & 96.1 & 95.9 & 96.0 & 89.4 & 90.5 & 90.0 \\
\hline $3: \odot 3: \odot \odot$ & 1623 & 93.0 & 92.3 & 92.7 & 95.0 & 95.9 & 95.5 & 89.4 & 90.5 & 90.0 \\
\hline $3: \odot 8$ & 628 & 93.0 & 92. & 92. & 95.0 & 95.9 & 95.5 & 89.4 & 90.5 & 90.0 \\
\hline $3: 13: \odot \odot$ & 1633 & 93 & 92 & & 95.0 & 95.9 & 95.5 & 89.4 & 90.5 & $9 \odot . \odot$ \\
\hline & & & & & & & & & 90.5 & $9 \odot . \odot$ \\
\hline $3: 23: \odot \odot$ & 1643 & 93.0 & 92.3 & 92.7 & 95.0 & 96.1 & 95.5 & 89.4 & 89.4 & 89.4 \\
\hline & 648 & & & & & & & 89.4 & 89.4 & 89.4 \\
\hline $3: 3$ & 1653 & 93.0 & 92.3 & 92.7 & 95.0 & 96.1 & 95.5 & 89.4 & 89.4 & 89.4 \\
\hline $3: 3 \varepsilon$ & 658 & 91.9 & 92. & 92. & & & & 89.4 & 89.4 & 89.4 \\
\hline $3: 43$ & 663 & 91.9 & 92. & 92.1 & 95.0 & 95 & 95.0 & 88.3 & 89.4 & 88.9 \\
\hline $3: 48$ & 668 & 91.9 & 92.3 & 92.1 & 93.9 & & 94.5 & 88.3 & 89.4 & 88.9 \\
\hline & & & 92.3 & 92.1 & & & 94.5 & 88.3 & 89.4 & 88.9 \\
\hline $3: 58: \odot \odot$ & 678 & 91.9 & 91.3 & 91.6 & 93.9 & 95.0 & 94.5 & 88.3 & 89.4 & 88.9 \\
\hline & & & & & & & & 88.3 & 89.4 & 88.9 \\
\hline $4: \odot 8: \odot \odot$ & 1688 & 91.9 & 91. & 91.6 & 93.9 & 95.0 & 94.5 & 88.3 & 89.4 & 88.9 \\
\hline $4: 13$ & 1693 & & & & & & & 88.3 & 88.3 & 88.3 \\
\hline & 1698 & 91.9 & 91. & 91.6 & & & & 88.3 & 88.3 & 88.3 \\
\hline $4: 23: \odot \odot$ & 1703 & 91.9 & 91.3 & 91.6 & & & & 88.3 & 88.3 & 88.3 \\
\hline & 1708 & 91.9 & 91.3 & 91.6 & & & & 88.3 & 88.3 & 88.3 \\
\hline $4: 33: \odot \odot$ & 1713 & & 91. & 91.6 & & & & 88.3 & 88.3 & 88.3 \\
\hline & 1718 & & & & & & & 87.3 & 88.3 & 87.8 \\
\hline & & & & & & 93.9 & 93.4 & 87.3 & 88.3 & 87.8 \\
\hline & & & 90.2 & 90.6 & 92.8 & & & 87.3 & 88.3 & \\
\hline
\end{tabular}




\begin{tabular}{|c|c|c|c|c|c|c|c|c|c|c|}
\hline $4: 53: 0 \odot$ & 1733 & 90.9 & 90.2 & 90.6 & 92.8 & 93.9 & 93.4 & 87.3 & 88.3 & 87.8 \\
\hline 4:58:๑๑ & 1738 & 90.9 & 90.2 & 90.6 & 92.8 & 93.9 & 93.4 & 87.3 & 88.3 & 87.8 \\
\hline $5: \odot 3: \odot \odot$ & 1743 & 90.9 & 90.2 & 90.6 & 92.8 & 93.9 & 93.4 & 87.3 & 88.3 & 87.8 \\
\hline $5: \odot 8: \odot \odot$ & 1748 & 90.9 & 90.2 & 90.6 & 92.8 & 93.9 & 93.4 & 87.3 & 88.3 & 87.8 \\
\hline $5: 13: \odot \odot$ & 1753 & 90.9 & 90.2 & 90.6 & 92.8 & 93.9 & 93.4 & 87.3 & 88.3 & 87.8 \\
\hline $5: 18: \odot \odot$ & 1758 & 90.9 & 90.2 & 90.6 & 92.8 & 93.9 & 93.4 & 87.3 & 88.3 & 87.8 \\
\hline $5: 23: \odot \odot$ & 1763 & 90.9 & 90.2 & 90.6 & 92.8 & 92.8 & 92.8 & 87.3 & 87.3 & 87.3 \\
\hline $5: 28: \odot \odot$ & 1768 & 90.9 & 90.2 & 90.6 & 91.8 & 93.9 & 92.8 & 87.3 & 87.3 & 87.3 \\
\hline $5: 33: \odot \odot$ & 1773 & 90.9 & 90.2 & 90.6 & 91.8 & 93.9 & 92.8 & 87.3 & 87.3 & 87.3 \\
\hline $5: 38: \odot \odot$ & 1778 & 90.9 & 90.2 & 90.6 & 91.8 & 92.8 & 92.3 & 86.2 & 87.3 & 86.7 \\
\hline $5: 43: \odot \odot$ & 1783 & 90.9 & 90.2 & 90.6 & 91.8 & 92.8 & 92.3 & 86.2 & 87.3 & 86.7 \\
\hline $5: 48: \odot \odot$ & 1788 & 89.8 & 90.2 & $9 \odot . \odot$ & 91.8 & 92.8 & 92.3 & 86.2 & 87.3 & 86.7 \\
\hline $5: 53: \odot \odot$ & 1793 & 89.8 & 90.2 & $90 . \odot$ & 91.8 & 92.8 & 92.3 & 86.2 & 87.3 & 86.7 \\
\hline $5: 58: \odot \odot$ & 1798 & 89.8 & 89.2 & 89.5 & 91.8 & 92.8 & 92.3 & 86.2 & 87.3 & 86.7 \\
\hline $6: \odot 3: \odot \odot$ & 1803 & 89.8 & 89.2 & 89.5 & 91.8 & 92.8 & 92.3 & 86.2 & 87.3 & 86.7 \\
\hline 6:๑8:๑๑ & 1808 & 89.8 & 89.2 & 89.5 & 91.8 & 92.8 & 92.3 & 86.2 & 87.3 & 86.7 \\
\hline $6: 13: \odot \odot$ & 1813 & 89.8 & 89.2 & 89.5 & 91.8 & 92.8 & 92.3 & 86.2 & 87.3 & 86.7 \\
\hline $6: 18: \odot \odot$ & 1818 & 89.8 & 89.2 & 89.5 & 90.7 & 92.8 & 91.8 & 86.2 & 87.3 & 86.7 \\
\hline $6: 23: \odot \odot$ & 1823 & 89.8 & 89.2 & 89.5 & 90.7 & 92.8 & 91.8 & 86.2 & 87.3 & 86.7 \\
\hline $6: 28: \odot \odot$ & 1828 & 89.8 & 89.2 & 89.5 & 90.7 & 92.8 & 91.8 & 86.2 & 87.3 & 86.7 \\
\hline $6: 33: \odot \odot$ & 1833 & 89.8 & 89.2 & 89.5 & 90.7 & 92.7 & 91.7 & 86.2 & 87.3 & 86.7 \\
\hline $6: 38: \odot \odot$ & 1838 & 89.8 & 89.2 & 89.5 & 90.7 & 92.7 & 91.7 & 86.2 & 87.3 & 86.7 \\
\hline $6: 43: \odot \odot$ & 1843 & 89.8 & 89.2 & 89.5 & 90.7 & 91.6 & 91.1 & 86.2 & 87.3 & 86.7 \\
\hline $6: 48: \odot \odot$ & 1848 & 89.8 & 89.2 & 89.5 & 90.7 & 91.6 & 91.1 & 86.2 & 87.3 & 86.7 \\
\hline $6: 53: \odot \odot$ & 1853 & 88.8 & 88.2 & 88.5 & 90.7 & 91.6 & 91.1 & 86.2 & 87.3 & 86.7 \\
\hline $6: 58: \odot \odot$ & 1858 & 88.8 & 88.2 & 88.5 & 90.7 & 91.6 & 91.1 & 86.2 & 86.2 & 86.2 \\
\hline $7: \odot 3: \odot \odot$ & 1863 & 88.8 & 88.2 & 88.5 & 90.7 & 91.6 & 91.1 & 86.2 & 86.2 & 86.2 \\
\hline 7:๑8:๑९ & 1868 & 88.8 & 88.2 & 88.5 & 90.7 & 91.6 & 91.1 & 86.2 & 86.2 & 86.2 \\
\hline $7: 13: \odot \odot$ & 1873 & 88.8 & 88.2 & 88.5 & 89.6 & 91.6 & 90.6 & 86.2 & 86.2 & 86.2 \\
\hline $7: 18: \odot \odot$ & 1878 & 88.8 & 88.2 & 88.5 & 89.6 & 91.6 & 90.6 & 86.2 & 86.2 & 86.2 \\
\hline $7: 23: \odot \odot$ & 1883 & 88.8 & 88.2 & 88.5 & 89.6 & 91.6 & 90.6 & 85.1 & 86.2 & 85.6 \\
\hline $7: 28: \odot \odot$ & 1888 & 88.8 & 88.2 & 88.5 & 89.6 & 91.6 & 90.6 & 85.1 & 86.2 & 85.6 \\
\hline $7: 33: \odot \odot$ & 1893 & 88.8 & 88.2 & 88.5 & 89.6 & 91.6 & 90.6 & 85.1 & 86.2 & 85.6 \\
\hline $7: 38: \odot \odot$ & 1898 & 88.8 & 88.2 & 88.5 & 89.6 & 90.5 & 90.1 & 85.1 & 86.2 & 85.6 \\
\hline $7: 43: \odot \odot$ & 1903 & 88.8 & 88.2 & 88.5 & 89.6 & $9 \odot .5$ & 90.1 & 85.1 & 86.2 & 85.6 \\
\hline $7: 48: \odot \odot$ & 1908 & 88.8 & 88.2 & 88.5 & 89.6 & 90.5 & 90.1 & 85.1 & 86.2 & 85.6 \\
\hline $7: 53: \odot \odot$ & 1913 & 88.8 & 88.2 & 88.5 & 89.6 & 90.5 & 90.1 & 85.1 & 86.2 & 85.6 \\
\hline $7: 58: \odot \odot$ & 1918 & 88.8 & 88.2 & 88.5 & 89.6 & 90.5 & 90.1 & 85.1 & 86.2 & 85.6 \\
\hline 8: $९ 3: \odot \odot$ & 1923 & 88.8 & 88.2 & 88.5 & 89.6 & 90.5 & 90.1 & 85.1 & 86.2 & 85.6 \\
\hline ৪:๑৪:๑९ & 1928 & 87.8 & 87.2 & 87.5 & 89.6 & 90.5 & 90.1 & 85.1 & 85.1 & 85.1 \\
\hline 8:13:๑९ & 1933 & 87.8 & 87.2 & 87.5 & 88.5 & 90.5 & 89.5 & 85.1 & 85.1 & 85.1 \\
\hline 8:18:๑९ & 1938 & 87.8 & 87.2 & 87.5 & 88.5 & 90.5 & 89.5 & 85.1 & 85.1 & 85.1 \\
\hline $8: 23: \odot \odot$ & 1943 & 87.8 & 87.2 & 87.5 & 88.5 & 90.5 & 89.5 & 85.1 & 85.1 & 85.1 \\
\hline $8: 28$ & 948 & 87.8 & 87.2 & 87.5 & 88.5 & 89.4 & 89.0 & 85.1 & 85.1 & 85.1 \\
\hline $8: 33$ & 1953 & 87.8 & 87.2 & 87.5 & 88.5 & 90.5 & 89.5 & 85.1 & 85.1 & 85.1 \\
\hline & & 87.8 & 87.2 & 87.5 & 88.5 & 89.4 & 89.0 & 85.1 & & 85.1 \\
\hline $8: 43: \odot \odot$ & 1963 & 87.8 & 87.2 & 87.5 & 88.5 & 89.4 & 89.0 & 85.1 & 85.1 & 85.1 \\
\hline ৪:48:๑९ & 1968 & 87.8 & 87.2 & 87.5 & 88.5 & 89.4 & 89.0 & 84.0 & 85.1 & 84.6 \\
\hline $8: 53: \odot \odot$ & 1973 & 87.8 & 87.2 & 87.5 & 88.5 & 89.4 & 89.0 & 84.0 & 85.1 & 84.6 \\
\hline $8: 58: \odot \odot$ & 1978 & 87.8 & 87.2 & 87.5 & 88.5 & 89.4 & 89.0 & 84.0 & 85.1 & 84.6 \\
\hline & 1983 & 87.8 & 87.2 & 87.5 & 88.5 & 89.4 & & 84.0 & & 84.6 \\
\hline 9:๑৪:๑๑ & 1988 & 87.8 & 87.2 & 87.5 & 88.5 & 89.4 & 89.0 & 84.0 & 85.1 & 84.6 \\
\hline & 1993 & 87.8 & 87.2 & 87.5 & 88.5 & 89.4 & 89.0 & 84.0 & 85.1 & 84.6 \\
\hline $9: 18: \odot \odot$ & 1998 & 87.8 & 86.2 & 87.0 & 87.4 & 88.3 & 87.9 & 84.0 & 85.1 & 84.6 \\
\hline $9: 23: \odot \odot$ & 003 & 87.8 & 86.2 & 87. & 87.4 & 88.3 & 87.9 & 84.0 & 85.1 & 84.6 \\
\hline & 08 & 86.7 & 86.2 & & 87.4 & 89.4 & 88.4 & 84.0 & 85.1 & 84.6 \\
\hline $9: 33: \odot \odot$ & 2013 & 86.7 & 86.2 & 86.5 & 87.4 & 89.4 & 88.4 & 82.9 & 84.0 & 83.5 \\
\hline $9: 38: \odot \odot$ & 2018 & 86.7 & & 86.5 & 87.4 & 88.3 & 87.9 & 82.9 & 84.0 & 83.5 \\
\hline $9: 43: \odot \odot$ & 2023 & 86.7 & 86.2 & 86.5 & 87.4 & 88.3 & 87.9 & 82.9 & 84.0 & 83.5 \\
\hline $9: 48: \odot \odot$ & 2028 & 86.7 & 86.2 & 86.5 & 87.4 & 88.3 & 87.9 & 82.9 & 84.0 & 83.5 \\
\hline $9: 53: \odot \odot$ & 2033 & 86.7 & 86.2 & 86 & 87.4 & 88.3 & 87.9 & 82.9 & 84.0 & 83.5 \\
\hline 9:58:๑९ & 2038 & 86.7 & 86.2 & & 87.4 & 88.3 & 87.9 & 82.9 & 84.0 & 83.5 \\
\hline $1 \odot: \odot 3: \odot \odot$ & 2043 & 86. & 86.2 & 86 & 87.4 & 88.3 & & 82.9 & 84.0 & 83.5 \\
\hline 1०: $\odot 8: \odot \odot$ & 2048 & 86.7 & 86.2 & 86.5 & 87.4 & 88.3 & 87.9 & 82.9 & 84.0 & 83.5 \\
\hline
\end{tabular}




\begin{tabular}{|c|c|c|c|c|c|c|c|c|c|c|}
\hline $10: 13: \odot \odot$ & 2053 & 86.7 & 86.2 & 86.5 & 87.4 & 88.3 & 87.9 & 82.9 & 84.0 & 83.5 \\
\hline $10: 18: \odot \odot$ & 2058 & 86.7 & 86.2 & 86.5 & 87.4 & 88.3 & 87.9 & 82.9 & 84.0 & 83.5 \\
\hline $10: 23: 00$ & 2063 & 86.7 & 86.2 & 86.5 & 86.4 & 88.3 & 87.4 & 82.9 & 84.0 & 83.5 \\
\hline $1 \odot: 28: \odot \odot$ & 2068 & 86.7 & 86.2 & 86.5 & 86.4 & 88.3 & 87.4 & 82.9 & 84.0 & 83.5 \\
\hline $10: 33: \odot \odot$ & 2073 & 86.7 & 85.2 & 85.9 & 86.4 & 88.3 & 87.4 & 82.9 & 84.0 & 83.5 \\
\hline $1 \odot: 38: \odot \odot$ & 2078 & 86.7 & 85.2 & 85.9 & 86.4 & 88.3 & 87.4 & 82.9 & 84.0 & 83.5 \\
\hline $10: 43: \odot \odot$ & 2083 & 85.6 & 85.2 & 85.4 & 86.4 & 87.3 & 86.8 & 82.9 & 82.9 & 82.9 \\
\hline $10: 48: \odot \odot$ & 2088 & 85.6 & 85.2 & 85.4 & 86.4 & 87.3 & 86.8 & 82.9 & 82.9 & 82.9 \\
\hline $10: 53: 0 \odot$ & 2093 & 85.6 & 85.2 & 85.4 & 86.4 & 87.3 & 86.8 & 82.9 & 82.9 & 82.9 \\
\hline $1 \odot: 58: \odot \odot$ & 2098 & 85.6 & 85.2 & 85.4 & 86.4 & 87.3 & 86.8 & 82.9 & 82.9 & 82.9 \\
\hline 11: ๑3: ๑९ & 2103 & 85.6 & 85.2 & 85.4 & 86.4 & 87.3 & 86.8 & 81.9 & 82.9 & 82.4 \\
\hline 11: ๑8: ๑९ & 2108 & 85.6 & 85.2 & 85.4 & 86.4 & 87.3 & 86.8 & 81.9 & 82.9 & 82.4 \\
\hline $11: 13: 0 \odot$ & 2113 & 85.6 & 85.2 & 85.4 & 86.4 & 87.3 & 86.8 & 81.9 & 82.9 & 82.4 \\
\hline $11: 18: 0 \odot$ & 2118 & 85.6 & 85.2 & 85.4 & 86.4 & 87.3 & 86.8 & 81.9 & 82.9 & 82.4 \\
\hline $11: 23: \odot \odot$ & 2123 & 85.6 & 85.2 & 85.4 & 86.4 & 87.3 & 86.8 & 81.9 & 82.9 & 82.4 \\
\hline $11: 28: \odot \odot$ & 2128 & 85.6 & 85.2 & 85.4 & 86.4 & 87.3 & 86.8 & 81.9 & 82.9 & 82.4 \\
\hline $11: 33: \odot \odot$ & 2133 & 85.6 & 85.2 & 85.4 & 85.3 & 87.3 & 86.3 & 81.9 & 82.9 & 82.4 \\
\hline $11: 38: 00$ & 2138 & 85.6 & 85.2 & 85.4 & 85.3 & 87.3 & 86.3 & 81.9 & 82.9 & 82.4 \\
\hline $11: 43: \odot \odot$ & 2143 & 85.6 & 85.2 & 85.4 & 85.3 & 87.1 & 86.2 & 81.9 & 82.9 & 82.4 \\
\hline $11: 48: \odot \odot$ & 2148 & 85.6 & 84.1 & 84.9 & 85.3 & 87.1 & 86.2 & 81.9 & 82.9 & 82.4 \\
\hline $11: 53: \odot \odot$ & 2153 & 85.6 & 84.1 & 84.9 & 85.3 & 87.1 & 86.2 & 81.9 & 82.9 & 82.4 \\
\hline $11: 58: \odot \odot$ & 2158 & 85.6 & 84.1 & 84.9 & 85.3 & 87.1 & 86.2 & 81.9 & 82.9 & 82.4 \\
\hline $12: \odot 3: \odot \odot$ & 2163 & 84.6 & 84.1 & 84.4 & 85.3 & 87.1 & 86.2 & 81.9 & 82.9 & 82.4 \\
\hline $12: \odot 8: \odot \odot$ & 2168 & 84.6 & 84.1 & 84.4 & 85.3 & 87.1 & 86.2 & 81.9 & 82.9 & 82.4 \\
\hline $12: 13: \odot \odot$ & 2173 & 84.6 & 84.1 & 84.4 & 85.3 & 86.0 & 85.6 & 81.9 & 81.9 & 81.9 \\
\hline $12: 18: \odot \odot$ & 2178 & 84.6 & 84.1 & 84.4 & 85.3 & 86.0 & 85.6 & 81.9 & 81.9 & 81.9 \\
\hline $12: 2$ & 2183 & 84.6 & 84.1 & 84.4 & 85.3 & 86.0 & 85.6 & 81.9 & 81.9 & 81.9 \\
\hline 12: & 2188 & 84.6 & 84.1 & 84.4 & 85.3 & 86.0 & 85.6 & 80.8 & 81.9 & 81.3 \\
\hline $12: 33: \odot \odot$ & 2193 & 84.6 & 84.1 & 84.4 & 85.3 & 86.0 & 85.6 & 80.8 & 81.9 & 81.3 \\
\hline $12: 38: \odot \odot$ & 2198 & 84.6 & 84.1 & 84.4 & 85.3 & 86.0 & 85.6 & 80.8 & 81.9 & 81.3 \\
\hline $12: 43: \odot \odot$ & 2203 & 84.6 & 84.1 & 84.4 & 84.2 & 86.0 & 85.1 & 80.8 & 81.9 & 81.3 \\
\hline $12: 48: \odot \odot$ & 2208 & 84.6 & 84.1 & 84.4 & 84.2 & 86.0 & & 80.8 & 81.9 & 81.3 \\
\hline $12: 5$ & 2213 & 84.6 & 84.1 & 84.4 & 84.2 & 86.0 & 85.1 & 80.8 & 81.9 & 81.3 \\
\hline $12: 58: \odot \odot$ & 2218 & 84.6 & 83.1 & 83.8 & 84.2 & 86.0 & 85.1 & 80.8 & 81.9 & 81.3 \\
\hline $13: 6$ & 2223 & 84.6 & 83.1 & 83. & 84 & 86.0 & 85.1 & 80.8 & 81.9 & 81.3 \\
\hline $13: \odot 8: \odot \odot$ & 2228 & 84.6 & 83.1 & 83.8 & 84.2 & 86.0 & 85.1 & 80.8 & 80.8 & 80.8 \\
\hline $13: 1$ & 2233 & 84.6 & 83.1 & 83.8 & 84.2 & 84.9 & 84.6 & 80.8 & 80.8 & 80.8 \\
\hline $13: 18: \odot \odot$ & 2238 & 84.6 & 83.1 & 83.8 & 84.2 & 84.9 & 84.6 & 80.8 & 80.8 & 80.8 \\
\hline 13: & 2243 & 83.5 & 83.1 & 83.3 & & & & & 80.8 & 80.2 \\
\hline $13: 2$ & 2248 & 83.5 & 83.1 & 83. & 84.2 & 84.9 & 84.6 & 79.7 & 80.8 & 80.2 \\
\hline & 2253 & 83.5 & 83.1 & 83 & & & & & 80.8 & 80.2 \\
\hline $13: 3$ & 2258 & 83.5 & 83.1 & 83. & 84.2 & 84.9 & 84.6 & 79.7 & 80.8 & 80.2 \\
\hline $13: 4$ & 2263 & 83.5 & 83.1 & 83.3 & 84.2 & 84.9 & 84.6 & 79.7 & 80.8 & 80.2 \\
\hline 13: & 2268 & 83.5 & 83.1 & 83. & 84.2 & 84.9 & 84.6 & 79.7 & 80.8 & 80.2 \\
\hline $13: 53: \odot \odot$ & 273 & 83.5 & 83.1 & 83 & 84.2 & 84.9 & 84.6 & 79.7 & 80.8 & 80.2 \\
\hline $13: 58: \odot \odot$ & & & & & & & & & & 80.2 \\
\hline $14: \odot 3: \odot \odot$ & 2283 & 83.5 & 83.1 & 83.3 & 84.2 & 84.9 & 84.6 & 79.7 & 80.8 & 80.2 \\
\hline & & & & & & & & & & 80.2 \\
\hline $14: 1$ & 2293 & 83.5 & 83.1 & 83.3 & 83.1 & 84.9 & 84.0 & 79.7 & 80.8 & 80.2 \\
\hline $14: 7$ & 2298 & 83.5 & 83.1 & 83.3 & & & & 79.7 & 80.8 & 80.2 \\
\hline & 2303 & 83.5 & 82.1 & 82.8 & & 84.9 & 84.0 & 79.7 & 80.8 & 80.2 \\
\hline $14: 2$ & 2308 & 83.5 & 82.1 & 82.8 & 83.1 & & & 79.7 & 80.8 & 80.2 \\
\hline & & & 82.1 & & & & & 79.7 & 80.8 & 80.2 \\
\hline $14: 3$ & 2318 & 82.5 & 82.1 & 82.3 & 83.1 & 84.9 & 84.0 & 79.7 & 80.8 & 80.2 \\
\hline & & & & & & & & & 80.8 & \\
\hline $14: 48: \odot \odot$ & 2328 & 82.5 & 82.1 & & & 84 & 84.0 & 9.7 & 80.8 & 80.2 \\
\hline $14: 53: \odot \odot$ & 2333 & 82.5 & 82.1 & & & & & 79.7 & 80.8 & 80.2 \\
\hline & 2338 & 82.5 & 82.1 & 82.3 & & & & 79.7 & 79.7 & 79.7 \\
\hline $15: \odot 3: \odot \odot$ & 2343 & 82.5 & 82.1 & 82.3 & & & & 79.7 & 79.7 & 79.7 \\
\hline & 2348 & 82.5 & 82.1 & 82.3 & & & & 79.7 & 79.7 & 79.7 \\
\hline $15: 13: \odot \odot$ & & 82.5 & 82.1 & & & & 83.5 & 79.7 & 79.7 & \\
\hline & & 82.5 & & & & & & 79.7 & 79.7 & 79.7 \\
\hline & & & & & & 83.8 & 83.5 & 79.7 & 79.7 & 79.7 \\
\hline & & & & 82.3 & 83 & 84.9 & 84.0 & 79.7 & 79.7 & \\
\hline
\end{tabular}




\begin{tabular}{|c|c|c|c|c|c|c|c|c|c|c|}
\hline $15: 33: 00$ & 2373 & 82.5 & 82.1 & 82.3 & 82.0 & 84.9 & 83.5 & 79.7 & 79.7 & 79.7 \\
\hline $15: 38: \odot \odot$ & 2378 & 82.5 & 82.1 & 82.3 & 82.0 & 84.9 & 83.5 & 78.6 & 79.7 & 79.2 \\
\hline $15: 43: \odot \odot$ & 2383 & 82.5 & 82.1 & 82.3 & 82.0 & 84.9 & 83.5 & 78.6 & 79.7 & 79.2 \\
\hline $15: 48: \odot \odot$ & 2388 & 82.5 & 81.1 & 81.8 & 82.0 & 84.9 & 83.5 & 78.6 & 79.7 & 79.2 \\
\hline $15: 53: \odot \odot$ & 2393 & 82.5 & 81.1 & 81.8 & 82.0 & 84.9 & 83.5 & 78.6 & 79.7 & 79.2 \\
\hline $15: 58: \odot \odot$ & 2398 & 82.5 & 81.1 & 81.8 & 82.0 & 84.9 & 83.5 & 78.6 & 79.7 & 79.2 \\
\hline $16: \odot 3: \odot \odot$ & 2403 & 82.5 & 81.1 & 81.8 & 82.0 & 84.9 & 83.5 & 78.6 & 79.7 & 79.2 \\
\hline $16: 08: \odot \odot$ & 2408 & 82.5 & 81.1 & 81.8 & 82.0 & 84.9 & 83.5 & 78.6 & 79.7 & 79.2 \\
\hline $16: 13: 00$ & 2413 & 81.5 & 81.1 & 81.3 & 82.0 & 84.9 & 83.5 & 78.6 & 79.7 & 79.2 \\
\hline $16: 18: \odot \odot$ & 2418 & 81.5 & 81.1 & 81.3 & 82.0 & 84.9 & 83.5 & 78.6 & 79.7 & 79.2 \\
\hline $16: 23: \odot \odot$ & 2423 & 81.5 & 81.1 & 81.3 & 82.0 & 84.9 & 83.5 & 78.6 & 79.7 & 79.2 \\
\hline $16: 28: \odot \odot$ & 2428 & 81.5 & 81.1 & 81.3 & 82.0 & 84.9 & 83.5 & 78.6 & 79.7 & 79.2 \\
\hline $16: 33: \odot \odot$ & 2433 & 81.5 & 81.1 & 81.3 & 82.0 & 84.9 & 83.5 & 78.6 & 79.7 & 79.2 \\
\hline $16: 38: 0 \odot$ & 2438 & 81.5 & 81.1 & 81.3 & 82.0 & 84.9 & 83.5 & 78.6 & 79.7 & 79.2 \\
\hline $16: 43: 00$ & 2443 & 81.5 & 81.1 & 81.3 & 82.0 & 84.9 & 83.5 & 78.6 & 79.7 & 79.2 \\
\hline $16: 48: 0 \odot$ & 2448 & 81.5 & 81.1 & 81.3 & 82.0 & 84.9 & 83.5 & 78.6 & 79.7 & 79.2 \\
\hline $16: 53: 00$ & 2453 & 81.5 & 81.1 & 81.3 & 82.0 & 83.8 & 82.9 & 78.6 & 78.6 & 78.6 \\
\hline $16: 58: \odot \odot$ & 2458 & 81.5 & 81.1 & 81.3 & 82.0 & 83.8 & 82.9 & 78.6 & 78.6 & 78.6 \\
\hline $17: 03: \odot \odot$ & 2463 & 81.5 & 81.1 & 81.3 & 82.0 & 83.8 & 82.9 & 78.6 & 78.6 & 78.6 \\
\hline $17: \odot 8: \odot \odot$ & 2468 & 81.5 & 81.1 & 81.3 & 82.0 & 83.8 & 82.9 & 78.6 & 78.6 & 78.6 \\
\hline $17: 13: 0 \odot$ & 2473 & 81.5 & 81.1 & 81.3 & 81.0 & 83.8 & 82.4 & 77.5 & 78.6 & 78.1 \\
\hline $17: 18: \odot \odot$ & 2478 & 81.5 & 81.1 & 81.3 & 81.0 & 83.8 & 82.4 & 77.5 & 78.6 & 78.1 \\
\hline $17: 23: \odot \odot$ & 2483 & 81.5 & 81.1 & 81.3 & 81.0 & 83.8 & 82.4 & 77.5 & 78.6 & 78.1 \\
\hline $17: 28: \odot \odot$ & 2488 & 81.5 & 80.1 & 80.8 & 81.0 & 83.8 & 82.4 & 77.5 & 78.6 & 78.1 \\
\hline $17: 33: 0 \odot$ & 2493 & 81.5 & 80.1 & 80.8 & 81.0 & 83.8 & 82.4 & 77.5 & 78.6 & 78.1 \\
\hline $17: 38: \odot \odot$ & 2498 & 81.5 & 80.1 & 80.8 & 81.0 & 83.8 & 82.4 & 77.5 & 78.6 & 78.1 \\
\hline
\end{tabular}

